\title{
Exploring the Molecular Mechanisms of Action
}

\author{
of Samoan Medicinal Plants
}

via Chemical Genetic Analyses

By

Seeseei Molimau-Samasoni

\begin{abstract}
A thesis
submitted to the Victoria University of Wellington in fulfilment of the requirements for the degree of

Doctor of Philosophy

in Cell and Molecular Bioscience
\end{abstract}

Victoria University of Wellington

2016 

This thesis was conducted under the supervision of

\section{Dr. Andrew B Munkacsi (Primary Supervisor) \\ Victoria University of Wellington \\ Wellington, New Zealand}

and

\section{Professor Paul H Atkinson (Secondary Supervisor) Victoria University of Wellington Wellington, New Zealand}





\section{Abstract:}

Natural products are a robust source of drug leads, and medicinal plants have been the source of natural products that are important pharmaceuticals in modern medicine. Samoan medicinal plants have been investigated in the past, but their potential as a source of new drug leads has not been realized. I hypothesized that determining the mechanism of action of Samoan medicinal plant extracts would provide insight into their pharmaceutical potential. The work described herein was carried out on 11 Samoan medicinal plants, from which 22 extracts were prepared. A bioactivity rate of $68 \%$ was determined when 15 of the 22 extracts inhibited the growth of yeast (Saccharomyces cerevisiae). The medicinal plant Psychotria insularum was the most potent, thus genome-wide analyses were completed using the haploid deletion mutant library of $S$. cerevisiae. Yeast strains deficient in iron transport were hypersensitive to the $P$. insularum aqueous extract. Further investigations showed that exogenous iron supplementation rescued the growth defect induced by $P$. insularum extracts, suggesting that $P$. insularum reduced intracellular iron. Fittingly, yeast cells treated with P. insularum extracts contained less intracellular iron than control cells. Paraxodically, the expression levels of iron transporter proteins were upregulated upon extract treatment. When we investigated iron-requiring cellular processes, we found that yeast cells treated with $P$. insularum extracts exhibited a respiratory deficient phenotype and reduced heme synthesis, indicative of an impaired cellular iron status. These findings suggested that $P$. insularum reduced bioavailable iron leading to the induction of the low iron response, and indeed the extracts of $P$. insularum were shown to chelate iron via the iron-chelating CAS assay. To translate results from yeast to mammalian cells, we treated primary murine macrophages with P. insularum extracts and detected an anti-inflammatory response, which we found to correlate with reduced activity of the iron-requiring aconitase enzyme. We further determined using pooled diploid mutant genetic analyses that the extracts of $P$. insularum did not have a genetic target. To identify the compound 
mediating the iron chelation mechanism, bioassay-guided isolation was conducted. Fractionation of the crude aqueous extract of $P$. insularum produced a relatively pure fraction that NMR and the acid-butanol assay identified as a condensed tannin. Together, these results indicate a relationship between iron chelation, a condensed tannin and inflammation. Further, we established an iron chelation mechanism of action by which $P$. insularum extracts are used to treat inflammationassociated symptoms in traditional Samoan medicine. Lastly, the findings presented here substantiate the reliability of plants with ethnobotanical background as sources for bioactive natural products. 


\section{Acknowledgements:}

This work could not have been completed without the tremendous help of numerous people, and I would like to express my most heartfelt gratitude to them. First and foremost, I would not be here without the funding from the Victoria Doctoral Scholarship, under the VUW and Scientific Research Organisation of Samoa (SROS) memorandum of cooperation (MOC). Thank you for the PhD opportunity! Thank you specifically to Afioga Tilafono David Hunter for the push to apply for this.

To my primary supervisor Dr Andrew Munkacsi: thank you for giving me the opportunity to study here by agreeing to take me on as one of your first PhD students, and for your endless support throughout my project. I do not think I could have come across a more patient supervisor than you! Prof. Paul Atkinson, your appreciation of my small victories was a constant reassurance that there was a method to the madness. I thank you both for giving me free reign over the direction of my project, because it has taught me the invaluable lesson of project design and critical thinking, important skills one would hope to walk away with from having completed a $\mathrm{PhD}$.

To the Chemical Genetics lab crew past and present, to say I have had a colorful experience working with you all would be an understatement. You have all contributed to a "culturally unique" experience during my studies in The Land of the Long White Cloud. I would especially like to thank Drs James Matthew, Peter Bircham and (pending Dr) Bede Busby for showing me the ropes around the lab. Peter and Bede, I'm pretty sure relaxing with you guys singing karaoke to Gangsta's Paradise led to a subsequent week of brain light bulbs lighting up! To my fellow PhD colleague Namal Coorey, our early morning lab sessions were one of my more productive lab times. We have yet to take a "morning crew" selfie! Dini, you are truly a jack of all trades. From pinning out library master copies to putting together a computer so I could write my thesis - You're a champ! To (pending Dr) Russian - Spasibo for the patient proof-reading; I know it was a painful task!

I also express my heartfelt gratitude to the other research groups that were involved in this work. In particular, I thank Dr Rob Keyzers, for your enthusiasm and for always having time to discuss the chemistry side of my project. Your help in deciphering my NMR spectra and your invaluable input to my chemistry chapter are sincerely acknowledged. To the student cohort in the chemistry labs: Benjamin Baker, Helen Woolner and Sarah Andreassand, (who helped out with running my LH20 column, TLCs and NMRs), your help in the chemistry lab is most appreciated. To Dr Daniel Sinclair from the School of Geography and Environmental Science, thank you for allowing 
us to run our samples on your ICP-MS, despite its limited use in this area. To Associate Professor Anne La Flamme, Pirooz Zareie and Vimal Patel, thank you for taking on the macrophage work on my extracts. Thanks also to Associate Professor David Ackerley and Dr. Jeremy Owen for your kind assistance and putting up with my troubleshooting of CAS assay hiccups. I would also like to acknowledge the help I received from Associate Professor David Gresham and his PhD student Darach Miller for the Bar-seq work carried out at NYU.

To the Samoan support group at SROS, from the staff, to the management and the Board, my most heartfelt Faafetai tele lava! You have supported me right from the start, through prayers, encouragement and financial assistance. I would not have been able to leave home and travel to NZ to undertake this without your support. To the Ministry of Natural Resources and Environment, specifically Mr Talie Foliga - A huge thank you for taking the time out to assist with my sample collection. I hope the findings of this research can contribute to your conservation efforts. Faamoetauloa Dr Faale Tumaalii, thank you for your faith in me.

It has been a challenge to undertake a PhD, away from the motherland. In this aspect, my husband, daughter and I have been the recipient of the kindness of many who we now consider family. Katie \& Viv Zeier, we thank you for your friendship and generosity - You have contributed to smoother sailing in the final year of my PhD. To the Teofilo, Lualua, Fruean and Poleta families, you took us in without any questions and your kind-heartedness will never be forgotten. To my dearest uncle Fitulua Utupo and his partner Virginia Fruean, we are unquestionably indebted to you both. From the moment we landed to our departure, you have been with us every step of the way. Through thick and thin, you have supported us unconditionally, and from the bottom of our hearts, we say THANK YOU!

Finally, to my dear husband and best friend, your endurance in raising our daughter by yourself for the most part of the time we've been here while I've been preoccupied with work is not unnoticed. Your endless support, prayers and encouragement has pushed me on to the finishing line. My dearest daughter Toeupu, I can only hope to provide you with a good example and a better future from this. Mum, thank you for encouraging me to take on this opportunity, and for pushing me to get the PhD I've always wanted. Last but not least, I dedicate this thesis to my late father Molimau Poleta - Dad, I’ve finally done it, Vi'ia le Ali'i! 


\section{Table of Contents:}

Abstract

Chapter 1: Literature Review

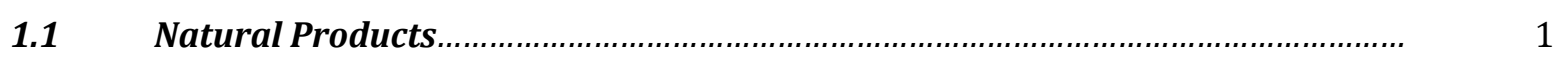

$1.2 \quad$ The status of natural products research over the years....................................... 2

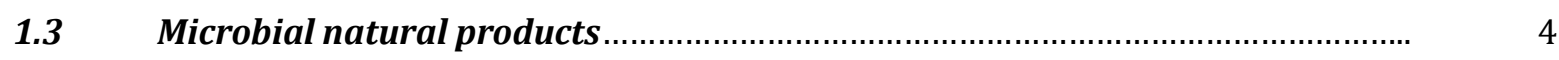

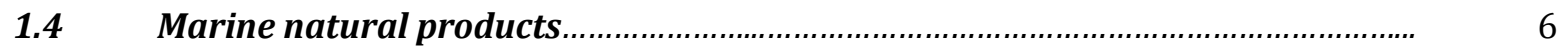

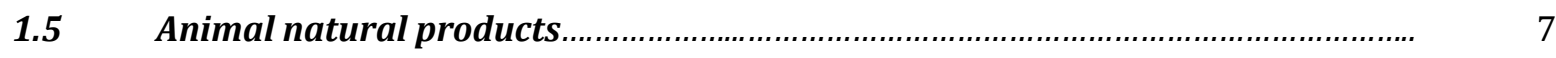

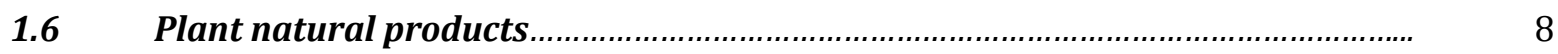

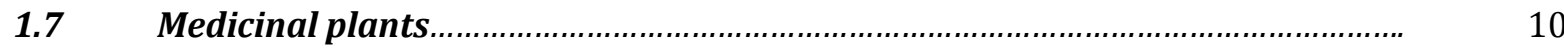

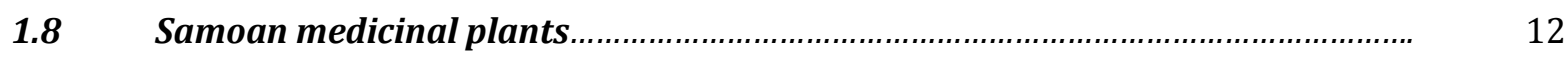

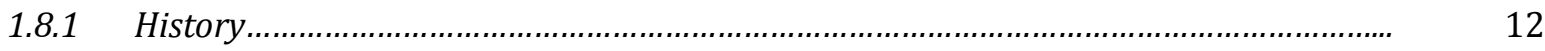

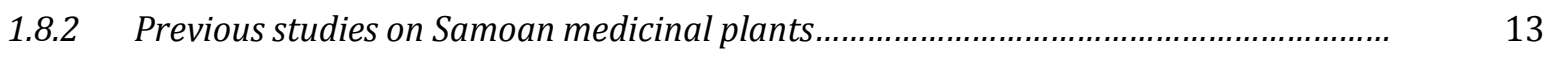

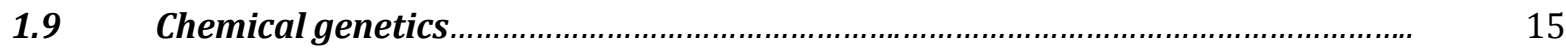

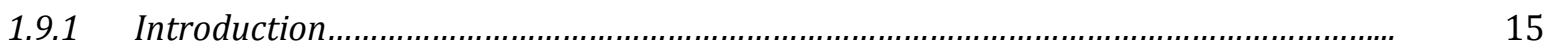

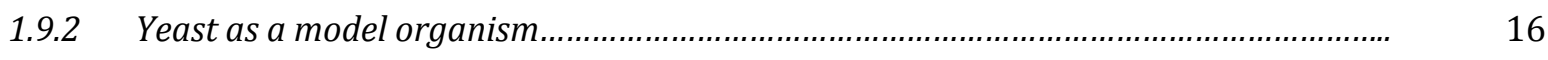

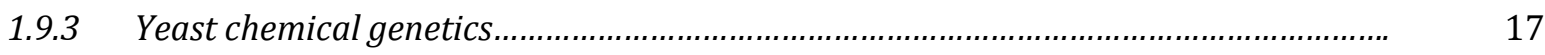

1.9.3.1 Characteristics of the gene deletion mutant collection ............................................. 17

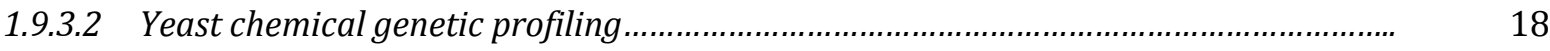

1.9.3.3 Drug targets \& mechanism of action via yeast chemical genetic profiling.................. $\quad 20$

1.9.3.4 Yeast chemical genetic profiling of crude natural product extracts............................

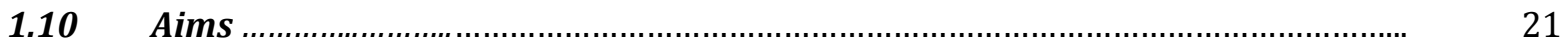




\begin{tabular}{|c|c|}
\hline 2.1 & Introduction.......... \\
\hline 2.1.1 & Bischofia javanica \\
\hline 2.1 .2 & Clerodendrum inerme \\
\hline 2.1.3 & Colubrina asiatica \\
\hline 2.1.4 & Cordyline fruticosa \\
\hline 2.1 .5 & Cymbobogon citratus \\
\hline 2.1 .6 & Flakourtia rukam \\
\hline 2.1 .7 & Macropiper puberulum \\
\hline 2.1 .8 & Psychotria insularum \\
\hline 2.1 .9 & Syzygium corynocarpum.... \\
\hline 2.1.10 & Vigna marina .................................... \\
\hline 2.1.11 & Wollastonia biflora \\
\hline 2.1 .12 & 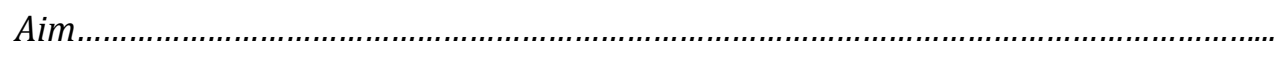 \\
\hline 2.2 & Methods \\
\hline 2.2 .1 & Medicinal plant collection \\
\hline 2.2 .2 & Extract preparation \\
\hline 2.2 .3 & 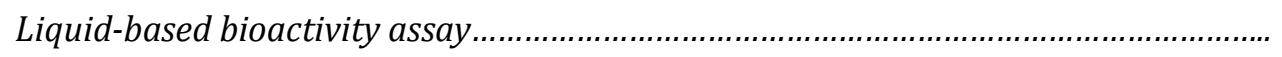 \\
\hline 2.2 .4 & Agar-based bioactivity assay \\
\hline 2.3 & Results \\
\hline 2.3.1 & 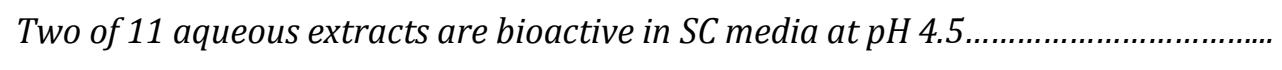 \\
\hline 2.3.2 & 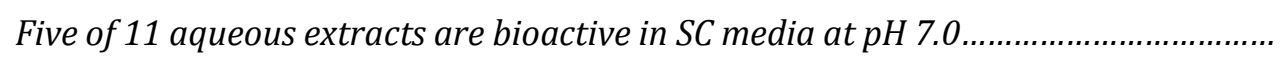 \\
\hline 2.3.3 & 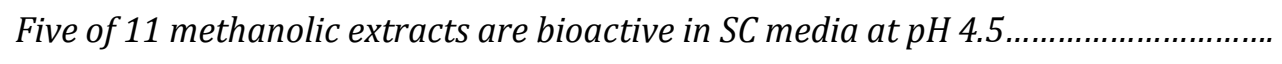 \\
\hline 2.3.4 & 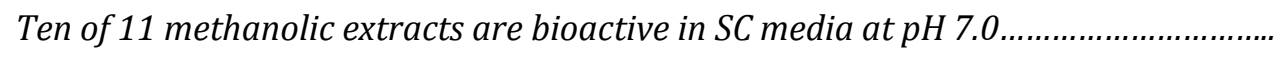 \\
\hline 2.3.5 & 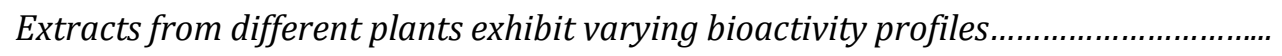 \\
\hline 2.3.6 & Aqueous extracts are not substrates of the PDR system \\
\hline 2.3.7 & Methanolic extracts are not substrates of the PDR system \\
\hline
\end{tabular}


2.3.8 Aqueous extracts maintain bioactivity in agar......................................................

2.3.9 Methanolic extracts maintain bioactivity in agar .......................................................

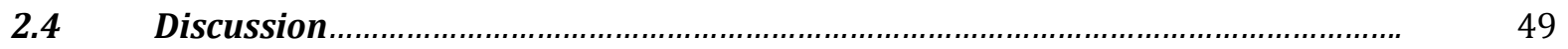

Chapter 3: Mechanism of Action of Psychotria insularum is iron chelation 52-93

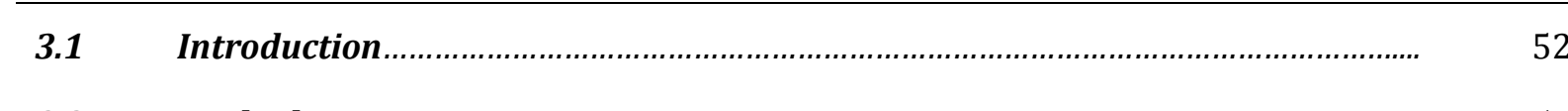

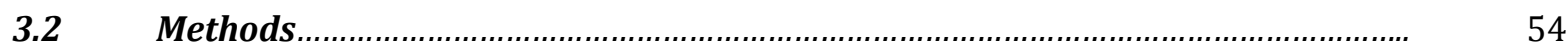

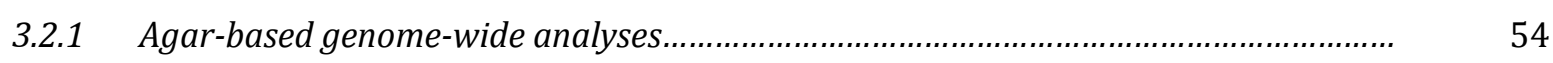

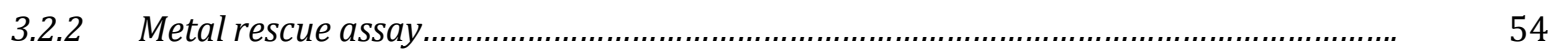

3.2.3 Quantification of intracellular iron ....................................................................

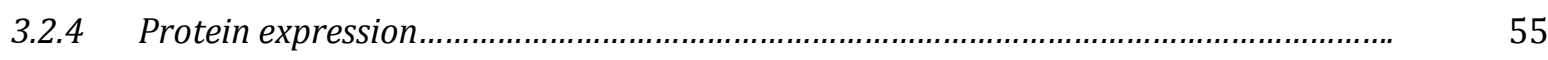

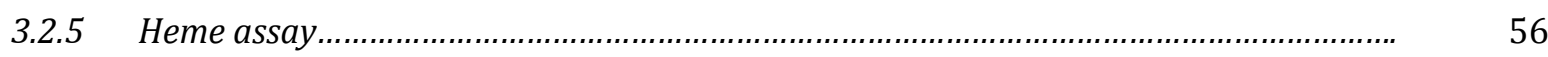

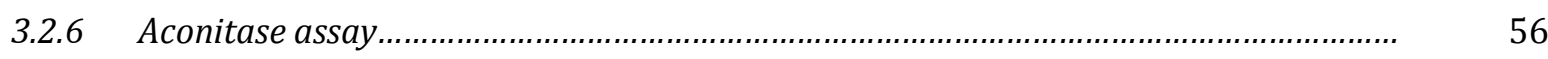

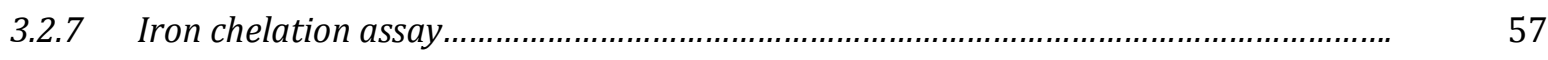

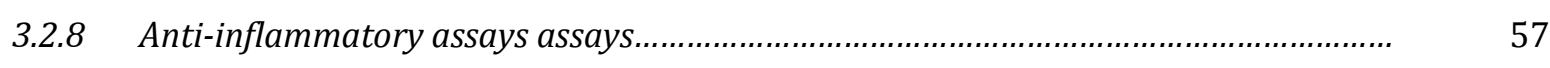

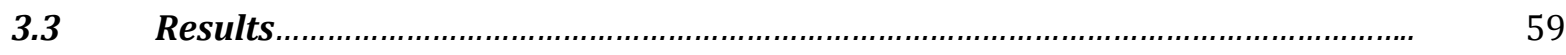

3.3.1 Genome-wide analysis of aqueous P. insularum extract.........................................

3.3.2 Validations implicate various biological processes in P. insularum MOA..................... 61

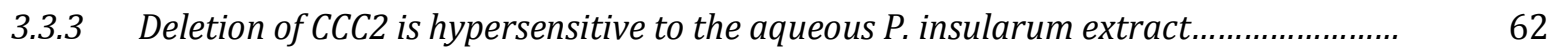

3.3.4 Gene deletions of the iron transport system are hypersensitive to the aqueous

3.3.5 Gene deletions of the iron transport system are hypersensitive to the methanolic extract of P. insularum....................................................................................... 66

3.3.6 Iron supplementation rescues the growth defect induced by the aqueous extract of

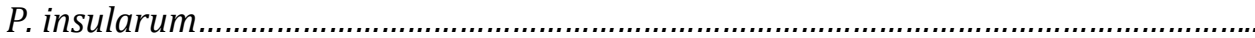

3.3.7 Iron rescue is specific to P. insularum extract treatments and is not a cytoprotective effect of iron supplementation

3.3.8 Supplementation with copper partially rescues the growth defect induced by $P$. insularum extracts in a Fet3p dependent manner..

3.3.9 Supplementation with other metals does not rescue the growth defect induced by extracts of P. insularum 
3.3.10 Intracellular iron is decreased in the presence of P. insularum extracts.......................

3.3.11 P. insularum extracts up-regulate the expression of iron transporters......................... 75

3.3.12 P. insularum extracts induce a respiratory deficient phenotype ................................... 79

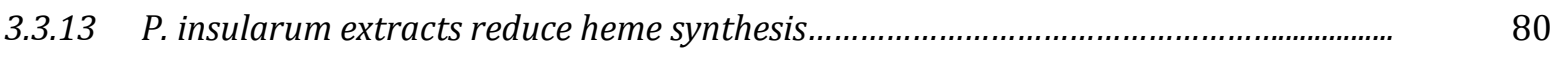

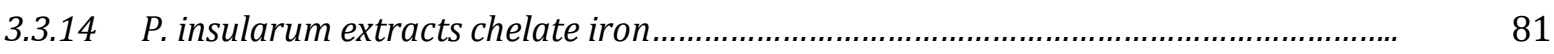

3.3.15 P. insularum extracts exhibit an anti-inflammatory response in murine

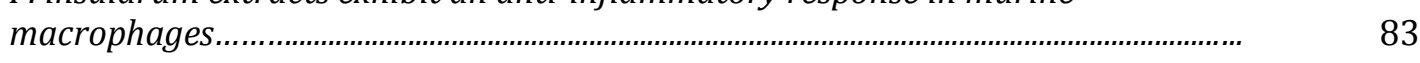

3.3.19 P. insularum extracts reduce aconitase activity in RAW 264.7

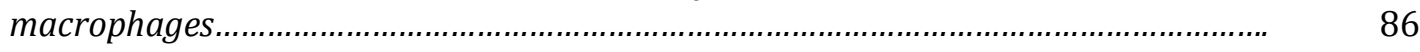

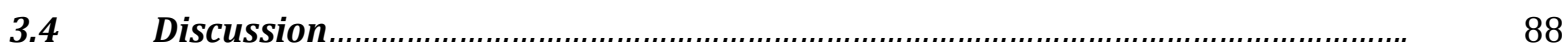

Chapter 4: Haploinsufficiency and Homozygous Profile Analyses of the Aqueous and 94-128 Methanolic Extracts of Psychotria insularum

\begin{tabular}{|c|c|c|}
\hline 4.1 & Introduction & 94 \\
\hline 4.2 & Methods & 96 \\
\hline 4.2 .1 & 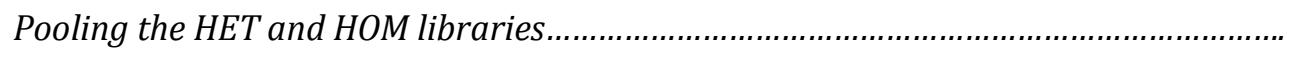 & 96 \\
\hline 4.2 .2 & 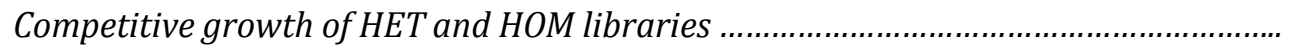 & 96 \\
\hline 4.2 .3 & Genomic DNA isolation & 97 \\
\hline 4.2 .4 & 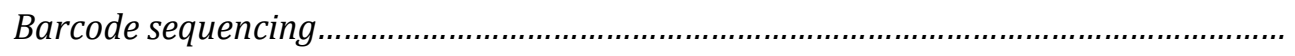 & 97 \\
\hline 4.2 .5 & 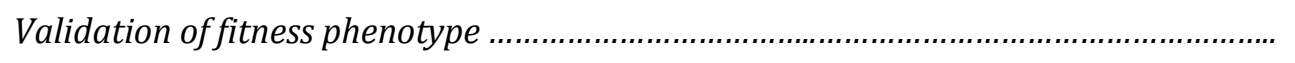 & 98 \\
\hline 4.3 & Results & 99 \\
\hline 4.3 .1 & 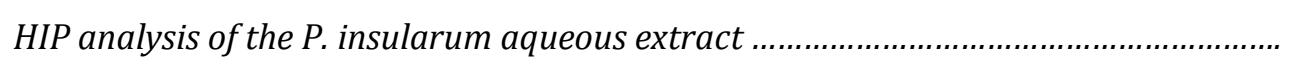 & 99 \\
\hline 4.3.2 & 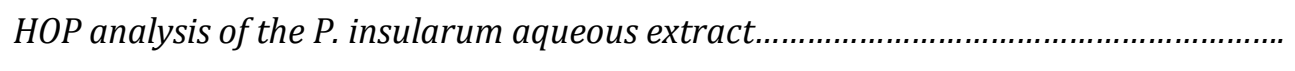 & 104 \\
\hline 4.3 .5 & 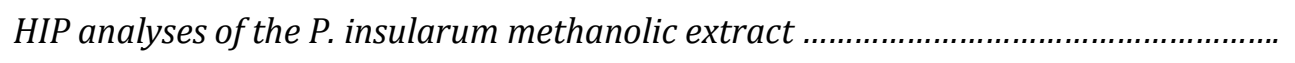 & 109 \\
\hline 4.3.7 & HOP analyses of the P. insularum methanolic extract & 114 \\
\hline 4.4 & Discussion & 124 \\
\hline
\end{tabular}

Chapter 5: Identification of the Iron Chelating Condensed Tannin from the Aqueous Extract of Psychotria insularum 
5.2 Methods

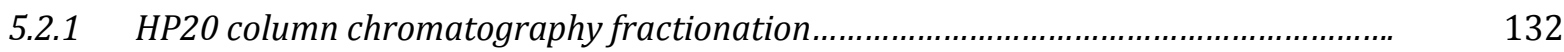

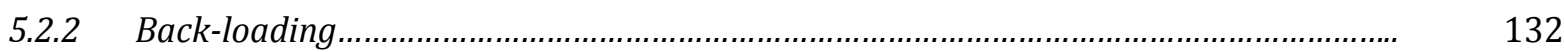

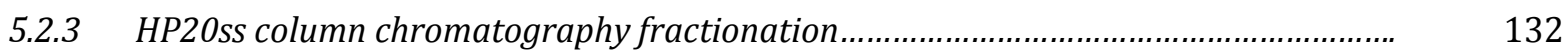

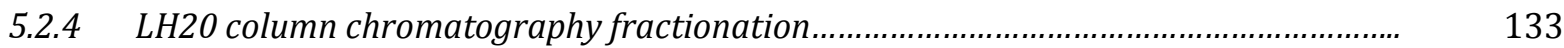

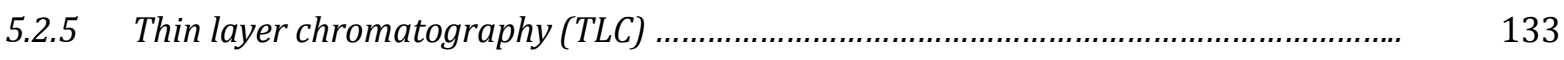

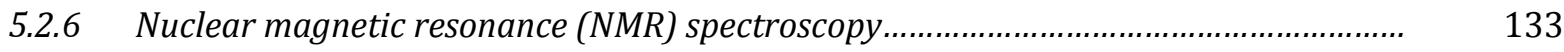

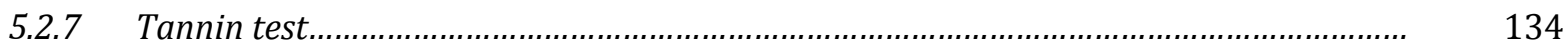

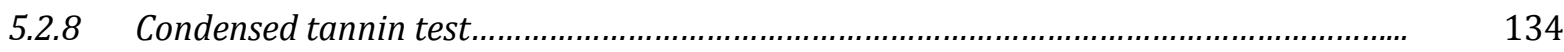

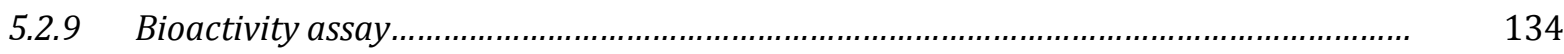

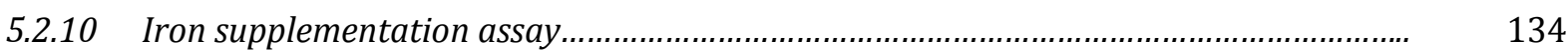

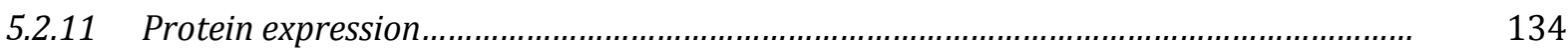

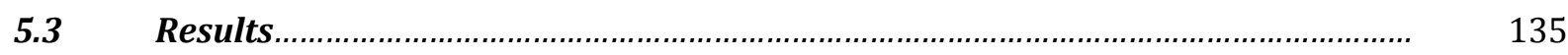

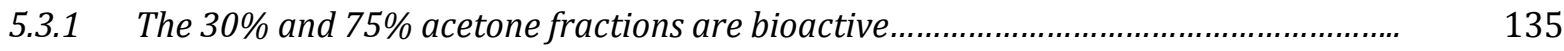

5.3.2 The ${ }^{1}$ H NMR analyses of the $30 \%$ and $75 \%$ fractions from HP20 fractionation show

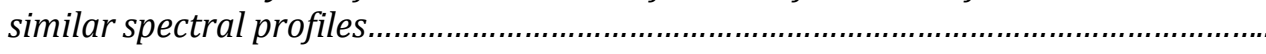

5.3.3 Subfractions of HP20ss fractionation of 30\% fraction are bioactive and variably potent

5.3.4 The NMR spectra of the 50\% and 100\% HP20ss fractions of the $30 \%$ aqueous extract fraction show highly similar profiles.

5.3.5 Subfractions of the HP20ss fractionation of the 75\% fraction are bioactive and variably potent.

5.3.6 NMR spectra of the 50\% and 60:100\% HP20ss fractions of the 75\% HP20 fraction display extremely similar profiles.

5.3.7 The 75-50\% LH20 pooled fractions A, E, F, G, H, I, K and P are not bioactive............... 143

5.3.8 The 75-50\% LH20 1L bulk fractions are bioactive ..................................................... 145

5.3.9 Expression of Fet3p and Ftr1p iron transporters are up-regulated in the presence of the selected bioactive fractions

5.3.10 Fraction $R$ from the LH20 column chromatography is a condensed tannin................... 149

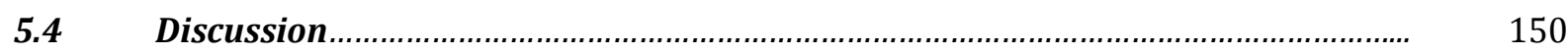




\begin{tabular}{|c|c|c|c|}
\hline 6.1 & \multicolumn{2}{|c|}{ Summary } & 155 \\
\hline 6.2 & \multicolumn{2}{|c|}{ 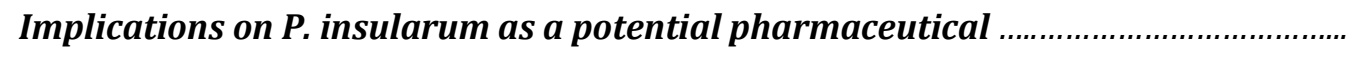 } & 155 \\
\hline 6.3 & \multicolumn{2}{|c|}{ Implications on P. insularum application in Samoan medicine } & 158 \\
\hline 6.4 & \multicolumn{2}{|c|}{ Implications on the P. insularum plant } & 159 \\
\hline 6.5 & \multicolumn{2}{|c|}{ Implications on natural products in Samoa } & 160 \\
\hline 6.6 & \multicolumn{2}{|c|}{ Future Directions } & 161 \\
\hline 6.7 & \multicolumn{2}{|c|}{ Conclusions } & 162 \\
\hline & \multicolumn{2}{|l|}{ References } & $163-189$ \\
\hline & Appendix I & Media Composition & I \\
\hline & Appendix II & Bioactivity of Aqueous Extracts at $70^{\circ} \mathrm{C}$ & II \\
\hline & Appendix III & Strains Genotypes & III \\
\hline & Appendix IV & List of Gene Deletions from Haploid Genetic Analysis & IV \\
\hline & Appendix $V$ & Spot Dilutions of all Hits & VII \\
\hline & Appendix VI & Script for Acapella GFP Quantification & IX \\
\hline & Appendix VII & Optimized Concentrations for HIP HOP Analyses & $\mathrm{X}$ \\
\hline & Appendix VIII & Universal Primer Sequence & $\mathrm{XI}$ \\
\hline & Appendix IX & ICP-MS Data for Zinc Quantification & XII \\
\hline
\end{tabular}




\section{List of Tables:}

Table 2.1 Summary of bioactivity of aqueous and methanolic extracts

Table 3.1 Optimization of aqueous extract concentration for genome-wide analyses

Table 4.1 The 25 hypersensitive heterozygotes against the aqueous extract of $P$. insularum.

Table $4.2 \quad$ The 26 heterozygotes that grew better in the aqueous extract of $P$. insularum...

Table 4.3 The 18 hypersensitive homozygous deletion mutants against the aqueous extract of $P$. insularum.....

Table 4.4 The 12 homozygous gene deletions that grew better in the presence of the aqueous $P$. insularum extract.

Table 4.5 The 20 hypersensitive heterozygotes resulting from treatment with the methanolic extract of $P$. insularum

Table 4.6 The 31 heterozygous gene deletions with positive $\operatorname{logFC}$ and FDR $<0.05 \ldots \ldots .$.

Table 4.7 Hypersensitive homozygous deletions against treatment with the methanolic extract of $P$. insularum.

Table 4.8 Top 90 gene deletions exhibiting improved growth in the presence of the methanolic extract of $P$. insularum. 


\section{List of Figures:}

Figure 1.1 New chemical entities from or based on natural products ................................. 2

Figure $1.2 \quad$ Original and total worldwide natural products patents, $1984-2003 \ldots \ldots \ldots \ldots \ldots \ldots . . . . . . .3$

Figure $1.3 \quad$ FDA-approved drugs of natural sources from 2008-2012 …............................. 4

Figure 1.4 New chemical entities based or derived from plant natural products............... 10

Figure 1.5 Construction strategy of the yeast deletion mutant collection............................ 18

Figure $1.6 \quad$ Chemical genetic interactions...................................................................... 18

Figure 2.1 Representations of the medicinal plants selected for this study ........................ 28

Figure 2.2 Location of collection of Samoan medicinal plants used in this study............... 29

Figure 2.3 Schematic of leaf juice sample preparation for analyses................................... 30

Figure 2.4 Liquid-based bioactivity assay of aqueous plant extracts in SC at $\mathrm{pH}$ 4.5.......... 34

Figure 2.5 Liquid-based bioactivity assay of aqueous plant extracts in $\mathrm{pH} 7.0 \mathrm{SC} . \ldots \ldots \ldots \ldots \ldots . . . . .36$

Figure 2.6 Liquid-based bioactivity assay of methanolic extracts in $\mathrm{pH} 4.5 \mathrm{SC} \ldots \ldots \ldots \ldots \ldots \ldots . . . . . . . . .38$

Figure 2.7 Liquid-based bioactivity assay of methanolic extracts in $\mathrm{pH} 7.0 \mathrm{SC} \ldots \ldots \ldots \ldots \ldots \ldots . . . . . . . . \quad 40$

Figure 2.8 Liquid-based growth assays of Y7092 WT and PDR-attenuated strain in

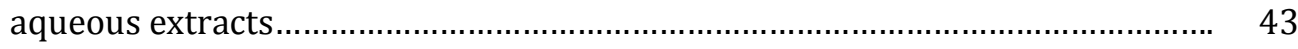

Figure 2.9 Liquid-based growth assays comparing growth profiles in WT and PDRattenuated strain in methanolic extracts........................................................ 45

Figure 2.10 Agar-based growth assays of WT in aqueous extracts ..................................... 47

Figure 2.11 Agar-based growth assays of WT against methanolic extracts......................... 48 
Figure 3.1 The scatterplot of gene deletion growth ratio against aqueous $P$. insularum

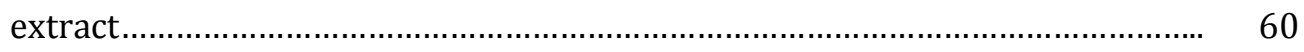

Figure 3.2 Spot dilution assay of five most hypersensitive gene deletions........................ 62

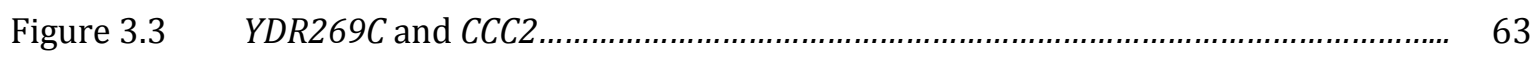

Figure 3.4 Growth profiles of gene deletions from the iron transport systems against the

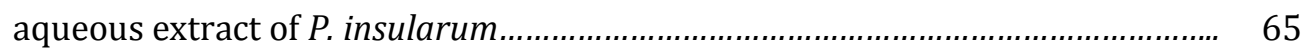

Figure 3.5 Growth profiles of gene deletions from the iron transport systems against the methanolic extract of $P$. insularum..............................................................

Figure 3.6 Iron supplementation against aqueous and methanolic extracts of $P$. insularum.

Figure 3.7 Iron supplementation of atorvastatin and cycloheximide drug treatment of BY4741 WT yeast

Figure 3.8 Copper supplementation rescue profiles of WT and fet $3 \Delta$ in the presence of aqueous and methanolic extracts of $P$. insularum.

Figure 3.9 Growth profiles of WT against aqueous and methanolic extracts of $P$. insularum with metal supplementation

Figure 3.10 Comparison of relative intracellular iron between control and treated WT...... 74

Figure 3.11 Expression levels of iron transporters under extract treatment monitored via GFP-tagged proteins

Figure 3.12 Quantification of GFP intensity of iron transporters upon P. insularum treatment

Figure 3.13 Respiratory deficient phenotype of BY4741...

Figure 3.14 Relative heme content of WT treated with P. insularum extracts.

Figure 3.15 CAS assay illustrating iron chelation activity of $P$. insularum extracts. 82

Figure 3.16 Macrophage response to treatment with P. insularum extracts 86 
Figure 3.17 The aconitase activity of macrophages

Figure 3.18 Model of the mechanism of action of $P$. insularum extracts.

Figure 4.1 Scatterplot of heterozygous mutants with false discovery rate values less than 0.05 against the aqueous extract of $P$. insularum, plotted as $\log \mathrm{CPM}$ vs $\log \mathrm{FC} . .$.

Figure 4.2 Heterozygous genes validated to be hypersensitive to the aqueous extract of P. insularum

Figure 4.3 Scatterplot of the 30 homozygous deletion mutants with false discovery rate values less than 0.05 against the aqueous extract of $P$. insularum, plotted as $\log \mathrm{CPM}$ vs $\log \mathrm{FC}$

Figure 4.4 Validations of the homozygous gene deletions detected to be hypersensitive to the aqueous extract of $P$. insularum

Figure 4.5 Scatterplot of the 51 heterozygous mutants with false discovery rate values less than 0.05 against the methanolic extract of $P$. insularum, plotted as $\log \mathrm{CPM}$ vs $\log \mathrm{FC}$

Figure 4.6 Validations of the heterozygote genes detected to be hypersensitive to the methanolic extract of $P$. insularum

Figure 4.7 Scatterplot of 288 homozygous mutants with false discovery rate values less than 0.05 against the methanolic extract of $P$. insularum plotted as $\log C P M$ vs $\log \mathrm{FC}$

Figure 4.8 Validations of the HOP hits of the methanolic extract of $P$. insularum

Figure 5.1 Schematic of bioassay guided fractionation

Figure 5.2 Bioassays of the $30 \%$ and $75 \%$ HP20 fractions of the aqueous $P$. insularum extract.

Figure 5.3 NMR spectra of the $30 \%$ and $75 \%$ HP2 20 fractions of the aqueous extract of $P$. 
Figure 5.4 Bioactivity and iron rescue assays of the HP20ss fractions of the 30\% HP20 fraction from the aqueous extract of $P$. insularum.

Figure 5.5 NMR spectra of the 50\% and 100\% HP20ss fractions from the 30\% HP20 fraction of the aqueous $P$. insularum extract

Figure 5.6 HP20ss fractions of the 75\% HP20 fraction from the aqueous extract of $P$. insularum.

Figure 5.7 NMR spectra of the 50\% and 60:100\% HP20ss fractions of the 75\% HP20 fraction of the aqueous extract of $P$. insularum

Figure 5.8 Bioactivity assay and NMR spectra of the pooled fractions from the LH20 fractionation of the 75-50 fraction from the aqueous extract of $P$. insularum.....

Figure 5.9 Bioassays and NMR spectra of the 75-50-Q and 75-50-R LH20 fractions.

Figure 5.10 Effects of the fractions leading to partially purified LH-20 bioactive fractions on the expression of the high affinity iron transporters Fet3p and Ftr1p ..........

Figure 5.11 Tannin and condensed tannin tests

Figure 5.12 Summary of the bioassay guided fractionation of the crude aqueous extract of P. insularum

Figure 5.13 Basic concept of polar fractionation of crude extract material. 


\section{Abbreviations:}

\begin{tabular}{|c|c|}
\hline ACE & Angiotensin converting enzyme \\
\hline AChE & Acetylcholinesterase \\
\hline $\mathrm{AD}$ & Alzheimer's Disease \\
\hline BBB & Blood brain barrier \\
\hline BPS & Bathophenanthroline disulfonic acid \\
\hline CAS & Chrome azurol S \\
\hline CBD & Convention of biological diversity \\
\hline CNS & Central nervous system \\
\hline DMSO & Dimethylsufoxide \\
\hline DFN & Deferiprone \\
\hline DFS & Deferasirox \\
\hline DFX & Deferroxamine \\
\hline ER & Endoplasmic reticulum \\
\hline FDA & Food and Drugs Administration \\
\hline FDR & False discovery rate \\
\hline GFP & Green fluorescence protein \\
\hline HDA & Histone deacetylase \\
\hline HIP & Haploinsufficiency profiling \\
\hline HIV & Human immunodeficiency virus \\
\hline HOP & Homozygous profiling \\
\hline ICP-MS & Inductively coupled plasma mass spectroscopy \\
\hline ISC & Iron sulfur cluster \\
\hline MOA & Mechanism of action \\
\hline
\end{tabular}




$\begin{array}{ll}\text { NCE } & \text { New chemical entity } \\ \text { NMR } & \text { Nuclear magnetic resonance } \\ \text { NP } & \text { Natural product } \\ \text { NPD } & \text { Natural product discovery } \\ \text { OD } & \text { Optical density } \\ \text { ORF } & \text { Open reading frame } \\ \text { PDR } & \text { Pleiotropic drug resistance } \\ \text { TCM } & \text { Traditional Chinese medicine } \\ \text { TLC } & \text { Thin layer chromatography } \\ \text { TOR } & \text { Target of rapamycin } \\ \text { WHO } & \text { World Health Organization } \\ \text { WT } & \text { Wild type }\end{array}$




\section{Chapter 1: \\ Literature Review}

\subsection{Natural Products}

Nature has provided a vast source of structurally diverse natural product compounds of therapeutic significance from plants, microbes and animals. These bioactive chemicals have endured selection pressures of evolution to regulate fundamental molecular pathways, making them ideal templates for pharmaceuticals. It is undeniable that natural products are the most successful source of new drugs, drug leads, and new chemical entities (NCEs) in today's pharmacopoeia. In the three decades spanning 1981-2010, 75\% of drugs approved by the United States Food \& Drug Administration (FDA) were either derived or based on natural products (Harvey et al., 2008). This is a testament to the reliability of natural products as drugs, or the basis of new drugs.

Historically, records of the use of natural products for medicinal purposes have been found as far back as 2900 BC (reviewed in Dias et al., 2012). This included documentation of more than 700-plant based treatments such as potions, ointments and pills. The use of oils from Cupressus sempevirens and Commiphora species was noted as far back as 2600 BC, and is still being used in modern day applications for the treatment of inflammation, colds and coughs. The Indian Ayurvedic medicinal system which describes the use of various herbs and dietary prescriptions for disease management in a holistic and systematic manner, evolved into a science in $2500 \mathrm{BC}$ and is still being used to date (Mishra et al, 2001). In addition, multiple Chinese records documenting more than 1,000 drugs and prescriptions containing herbs and plants for traditional medicines exist from various periods including 1100 BC (Chinese Materia Medica), 100 BC (Shennong Herbal) and 659 AD (the Tang Herbal) (Dias et al., 2012). 


\subsection{The status of natural products research over the years}

The fortuitous discovery of penicillin from the filamentous fungus Penicillium notatum in 1928 spearheaded the natural products era, leading to pharmaceutical companies establishing individual natural products discovery programs. However, this area of research started waning in the 1990s due to several problems associated with natural products research, such as the lengthy period it takes from discovery to marketing, and issues involved with procuring sufficient material for studies and market supply.

Historically, natural products contributed close to half of NCEs in the six (6) decades spanning 1931-1990 (Fig 1.1, Patridge et al., 2015). Specifically, in the decades between 1931-1940, and 1971-1980, natural products contributed to more than half of NCEs. However, since the 1990's, the contribution of natural products to NCEs decreased to a third, which can be attributed to the reasons mentioned above. More rigorous testing in relation to product safety may also have contributed to this decline, as well as the general movement away from natural products in the 1990s.

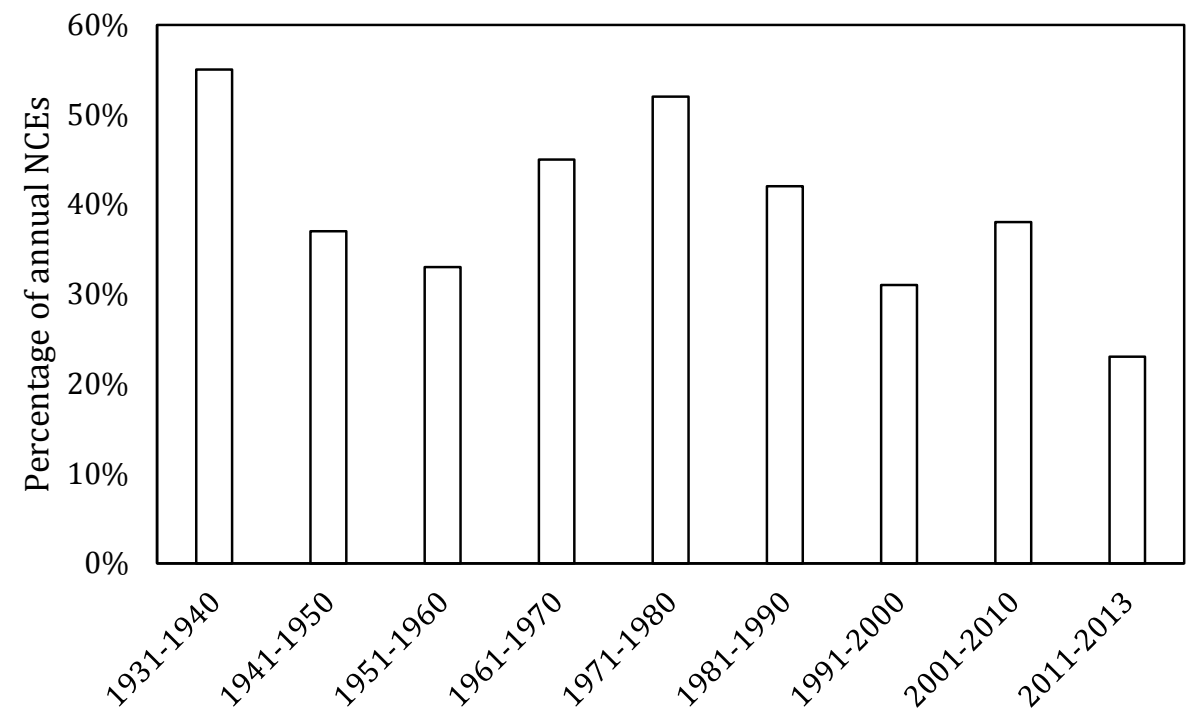

Figure 1.1: New chemical entities from or based on natural products. The percentage of approved new chemical entities (NCEs) derived or based on natural products, of microbial, plant or animal origin, for the decades between 1931-2013 (Adapted from Patridge et al., 2015). 
Another way to monitor the advances on natural products and their associated NCEs is an investigation of patents relating to such products. Koehn \& Carter (2005) found that despite reported reduction in NCEs arising from natural products, the case was the opposite with registered patents on small molecule natural products, for the 20-year period from 1984-2003 (Fig 1.2). A comparison between 1984 and 2003 showed more than five-fold increase in total worldwide natural-product patents, and a three-fold increase in original natural-product patents, indicative of the advances in synthetic chemistry and the production of semi-synthetic derivatives and analogues of original natural product molecules (Koehn \& Carter, 2005). Despite the variable number of patents on a year-by-year basis, a general increasing trend in total worldwide natural product patents is observed, thus painting a promising picture regarding natural product research.

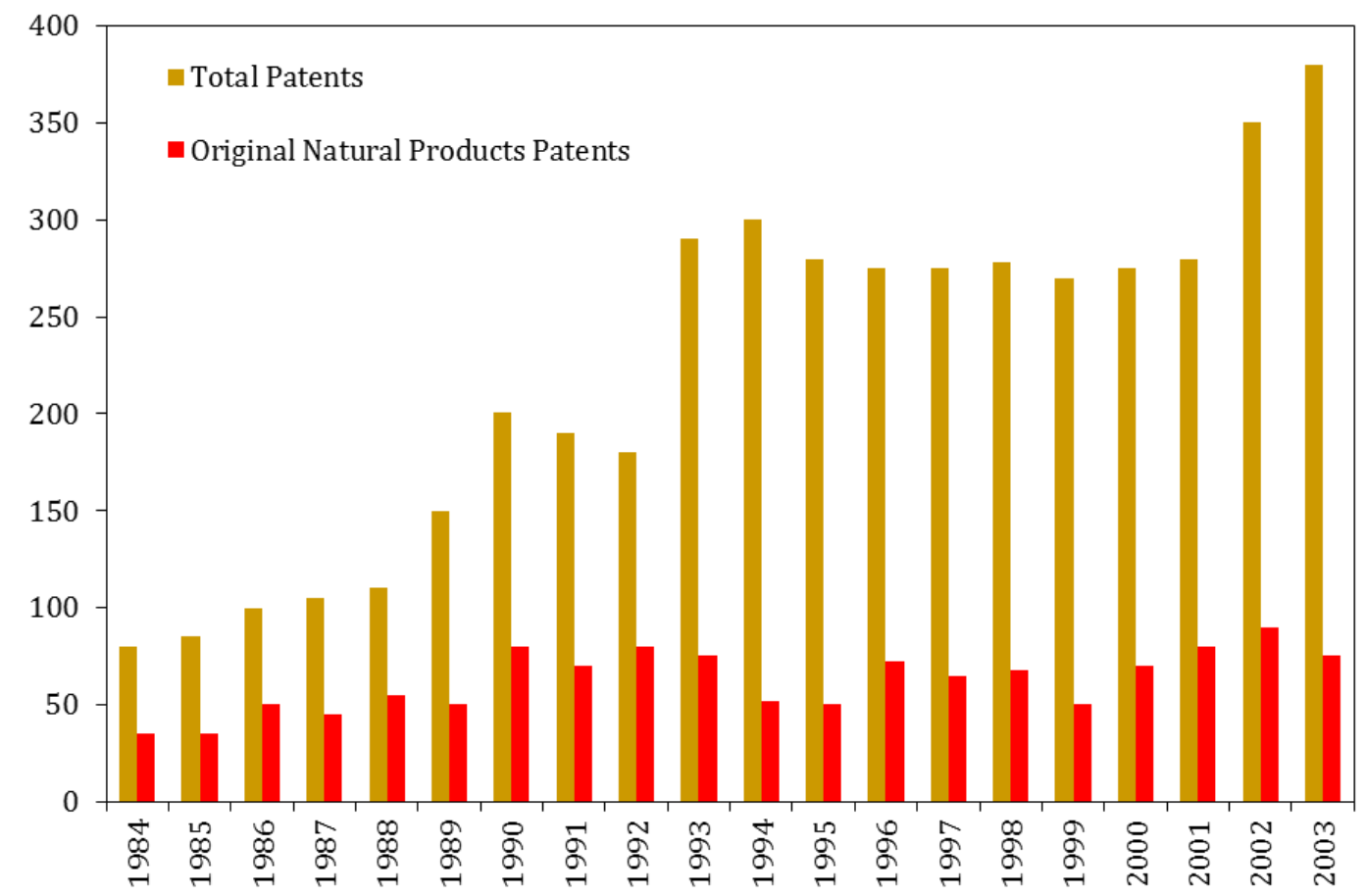

Figure 1.2: $\quad$ Original and total worldwide natural product patents, 1984-2003. Total worldwide natural product patents shown in gold represent patents of small molecules composed of (either partially or wholly) natural products as pharmaceuticals. Original natural product patents are shown in orange, and denote first-time novel composition of small molecular natural products as pharmaceuticals (Adapted from Koehn \& Carter 2005). 
However, given that a significant number of chemicals and molecules filed for patents do not pass through drug development and trials, a measure of the reliability of natural products for the production of new drugs is the number of new FDA approved drugs in recent years. Tao et al. (2014) noted that products of natural sources accounted for approximately $35 \%$ of FDA-approved drugs in 2008 , whilst in 2010 , the figure was approximately $80 \%$ (Fig 1.3). Drugs derived or based on natural sources contributed to approximately 50\% of all FDA-approved drugs in both 2011 and 2012 (Tao et al., 2014). These recent data continue to support a primacy of natural products in drug discovery, added to by advances in screening strategies and augmentation of existing traditional methods (Harvey et al., 2015).

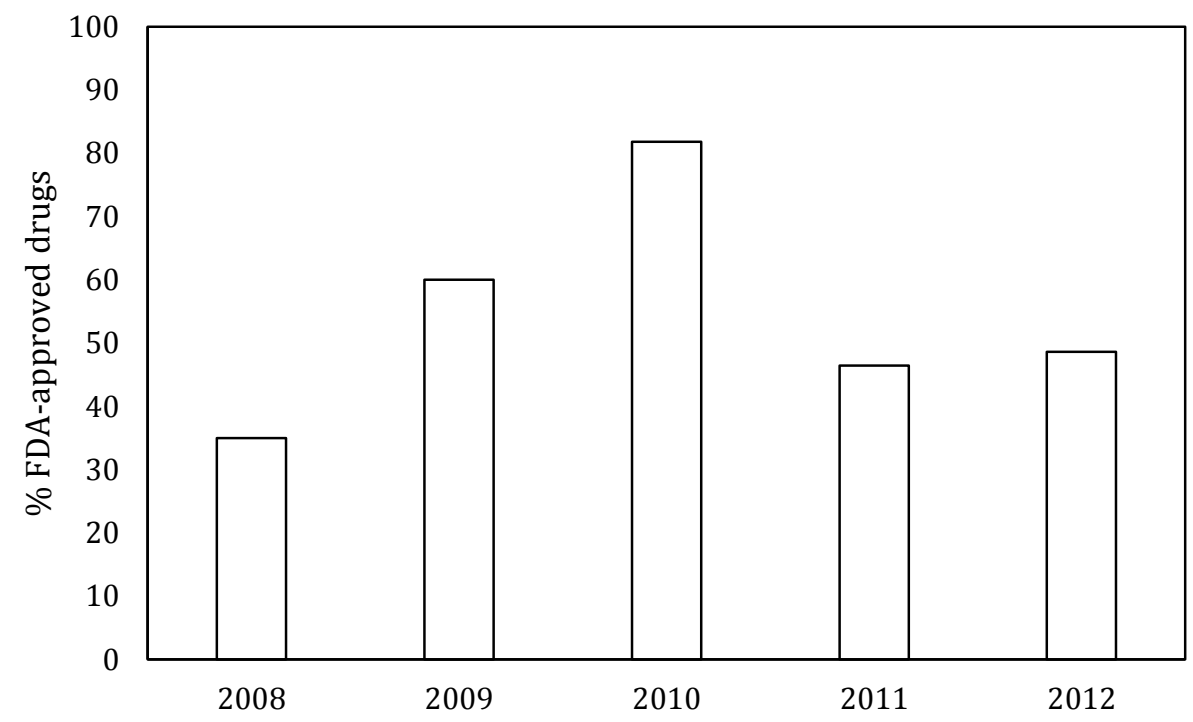

Fig1.3: $\quad$ FDA-approved drugs of natural sources from 2008-2012. Percentage of FDAapproved drug of natural sources, including natural product drugs and drugs based on natural products of microbial, marine, plant, and animal sources, for the years 2008-2012 (Adapted from Tao et al., 2014).

\subsection{Microbial Natural Products}

The opportune discovery of penicillin from the filamentous fungus Penicillium notatum drove investigations into microorganisms as sources of drugs, and subsequently the "Golden Age of Antibiotics", which was an era marked by exponential discoveries of antibiotics from natural 
products, particularly of microbial sources. However, in spite of its revolutionary effect on drug discovery and natural product research, penicillin was not effective against all bacteria, and subsequent antibiotic resistance led to chemical modifications to the underlying penicillin structure, giving rise to more potent and wider spectrum antibiotics (Miller, 2002).

Tetracycline, another well-known antibiotic was isolated from the bacterium Streptomyces aureofaciens. As one of the first successful semi-synthetic pharmaceutical products derived from a natural product, tetracycline was produced from modifications to chlortetracycline (Aureomycin) by removal of its C7 chlorine. Indeed, the underlying naphthacene structure of chlortetracycline has been the basis of second generation tetracyclines, such as doxycycline (Vibramycin) (Nelson \& Levy, 2011). Doxycycline is used as a broad spectrum antibiotic against community-acquired bacterial infections, malaria and Bacillus anthracis, the causative agent of anthrax infections (Nelson \& Levy, 2011).

The bacterium Streptomyces hygroscopicus is the source of rapamycin, a well-known immunosuppressive agent used to reduce organ rejection in transplant cases. A secondary metabolite of S. hygroscopicus, rapamycin was first isolated in the 1970s, and was found to have antifungal, antitumor and immunosuppressive activities (Benjamin et al., 2011). Interestingly, it was its antifungal activity that facilitated the discovery of the central regulatory molecule TOR (target of rapamycin), which in turn facilitated better understanding of TOR and its mechanisms (Benjamin et al., 2011).

Cholesterol-lowering statin drugs were also sourced from microorganism NPs. Following the successful use of lovastatin in the clinical setting in the 1980s, two semi-synthetic statins (simvastatin and pravastatin) and four synthetic statins (fluvastatin, atorvastatin, rosuvastatine and pitavastatin) have been introduced and successfully used in lowering cholesterol levels (Endo, 2010). In addition, the extensively used anticancer drugs bleomycin $A_{2}$ and $B_{2}$ are also of a microorganism source, from the bacterium Streptomyces verticillus (Cragg \& Newman, 2013). 
In 2015, half of the Nobel Prize for Physiology or Medicine was awarded jointly to William C Campbell and Satoshi Ōmura for their discovery of avermectins that are produced solely by the bacterium Streptomyces avermitilis (Ōmura \& Crump, 2004; Nobel Assembly, 2015). This species was first isolated in Japan 1973, and its anti-parasitic activity was detected in 1974. Later in the 1970s, the anti-helminthic activity of avermectin was discovered in the USA (Ōmura \& Crump, 2004). Avermectin was subsequently found to have a broad range of anti-parasitic activity, with a highly potent and unique mechanism of action. A modification of the natural avermectins gave rise to a broader spectrum and less toxic compound that was marketed as ivermectin (Campbell, 2012). Ivermectin has since been used in 46 countries to treat cattle, sheep, horses and pigs; in New Zealand, avermectin is a front-line treatment for sheep roundworms without which the sheep industry would have difficulty surviving. Most importantly however, avermectin is used to treat river blindness and elephantiasis in humans and is undergoing trials for an ever-increasing number of applications against other parasitic diseases (Ōmura \& Crump, 2004).

\subsection{Marine Natural Products}

The oceans, which cover approximately $70 \%$ of the earth's surface, are another source of natural products. Whilst investigations into marine environments only started in the mid-1970s, multiple structures have been discovered since. For instance, 2,500 new metabolites were reported from 1977-1987, whilst up to 1,003 new compounds were recorded for the year 2010 alone (Blunt et al., 2012; Faulkner, 2000). These results are indicative of the marine environment being a prolific source of natural products and NCEs.

Ziconotide, the first marine derived product to be approved as a drug was isolated from the venom used by the cone snail Conus magus to facilitate capture of its prey (Cragg \& Newman, 2013); the drug is used for the management of severe chronic pain through intrathecal application (McGivern, 2007). Of particular note is its unique mechanism of action; ziconotide is the only clinically approved selective N-type channel (calcium channel) blocker (McGivern, 2007). 
The alkaloid ecteinascidin 743, 40 years after its isolation from Ecteinascidia turbinate, was the first approved marine-derived anticancer drug (Molinski et al., 2009). It has been approved for the treatment of soft tissue sarcomas, and is currently undergoing various phases of clinical trials for the treatment of breast, prostate and pediatric sarcomas (Cragg \& Newman, 2013).

Additional marine products with anticancer activity undergoing clinical trials are halichondrin B and its analogue eribulin mesylate (Cragg \& Newman, 2013). Halichondrin B was discovered in 1986, but owing to limited compound availability as is common with natural products, its progress was delayed. Total synthesis of halichondrin B required 90 steps, however, further research found active synthetic analogues of halichondrin B with $30 \%$ less molecular mass that exhibited broad anti-proliferative activity against tumour cells, similar to that of halichondrin B (Towle et al., 2001).

\subsection{Animal Natural Products}

Drugs produced from natural sources also include those obtained from animal sources, although research on animal natural products is dwarfed in comparison to the volume of studies conducted on natural products from plant and microbial sources (Paavilainen, 2009). Whilst research on animal natural products is increasingly becoming more common, a significant number of these "medicinal" animals are listed as threatened species, largely due to their exploitation as a result of their use in traditional medicine (Alves \& Albuquerque, 2013). While it is generally accepted that compounds insulin, trypsin and other human hormones are important animal products used for therapeutic applications, this section will present some examples of natural products discovered and isolated from animal sources that successfully entered the market for clinical use, or are currently undergoing trials for introduction into the pharmaceutical market.

The angiotensin converting enzyme (ACE) inhibitors captopril and enalapril were synthesized on the basis of the nonapeptide teprotide isolated from the venom of the Brazilian pit viper Bothrops jararaca in the 1970s (Ferreira et al., 1970; Ondetti et al., 1971; Ondetti et al., 1973). 
This was a major breakthrough for antihypertension medications. However, whilst teprotide exhibited significant hypotension activity, its lack of oral activity led to the development of captopril and enalapril, which are used to treat not only hypertension but also congestive heart failure and diabetic nephropathy (Cushman \& Ondetti, 1999).

The alkaloid epibatidine that was isolated from the skin of the poisonous frog Epipedobates tricolor forms the basis of the development of a novel class of potential painkillers (Spainhour, 2005). Additionally, the development of the injectable glucose extenatide polypeptide was based on exendin-4 isolated from venom of the Gila monster Heloderma suspectum (Eng et al., 1992). Further to these drugs on the market, natural products from animals have also shown promise through antimicrobial peptides such as cecropins and defensins (Aerts et al., 2008; Boman, 1991). Various bee products such as honey, venom and propolis (bee glue) have also showed varied activities, including but not limited to anti-cancer and anti-HIV properties (Molan, 1999; Park et al., 2000; Wade et al., 1992).

\subsection{Plant Natural Products}

Plants have played a significant contribution in the field of drug discovery. Whilst it is understood that plants have contributed to medicine as far back as 2900 BC, the earliest contribution of plants into modern medicine started with the discovery of digoxin from Digitalis purpurea (foxglove) in 1785 . William Withering was first made aware of the plant's medicinal properties from patients who received a potion containing D. purpurea (Aronson, 1977). Since then, other Digitalis spp. have contributed their own chemical compounds to medicine such as acetyldigoxin, digitalin, digitoxin, deslanoside, and lanatosides A, B and C from D. lanata (Lahlou, 2013).

The bark of Cinchona trees was often chewed by South American natives to abate shivering while working in cold waters and to treat fevers (Meshnick \& Dobson, 2001). Later in the 1600s, its antimalarial properties were accidently discovered (Kaufman \& Rúveda, 2005). Pure 
quinine and cinchonine were later isolated from Cinchona bark in 1820 in Europe, and thereafter used by physicians to treat malaria (Meshnick \& Dobson, 2001). Whilst references were made to an opium elixir with pain-relieving properties in the BC period, morphine was first isolated from Papaver somniferum in the early 1800s by the German pharmacist Friedrich Sertürner (Brune \& Hinz, 2004). The P. somniferum extract also contained codeine (another analgesic), and papaverine (a muscle relaxant) which reduced cardiac muscle contraction (Davies \& Hollman, 2002). A synthetic analogue of papaverine called verapamil was shown to be highly active as a calcium channel blocker, in its activity against arrhythmia (Davies \& Hollman, 2002). Noscapine, another component of opium, is used as a cough suppressant and has also been shown to have anti-cancer activity (Mahmoudian \& Rahimi-Moghaddam, 2008). An effort by the National Cancer Institute screening a large collection of plant extracts led to the discovery of the widely-used anti-cancer drug paclitaxel in 1971, from the Pacific yew tree, Taxus brevifolia (Rowinsky \& Donehower, 1995). Other contributions of plants into medicine include vinblastine and vincristine from the Madagascar periwinkle Catharanthus roseus, and etoposide and teniposide-based on epipodophyllotoxin isolated from the root of the American mayapple plant Podophyllum peltatum (Itokawa et al., 2008).

Nevertheless, despite these promising leads, plant natural products have shown a decline in recent years (Fig 1.4, Patridge et al., 2015). Whilst they contributed to $15-20 \%$ of total approved annual NCEs in the three decades spanning 1931-1960, plant natural products contributed to less than $10 \%$ of total annual NCEs discovery from 1961-2013. In addition to the factors previously discussed (Section 1.1), another factor that may have contributed to this decline in plant natural product outputs was the Convention of Biological Diversity and the regulations it put in place to protect biodiversity and sustainability. Additionally, it is proposed that discoveries from plant natural products have slowed down because the "lower hanging fruit" of plant natural products have been picked, and what remains requires more perseverance, time and financial investment. Contrastingly, there was an increase in NCE/year, from 0.6 NCE/year from 2001-2010 to 1.5 NCE/year in the period 2011-2013 (Fig 1.4) suggesting that when effort is expended, plenty of plant NCEs remain to be 
discovered. This prospect is broadened since $90 \%$ of species in the plant kingdom have yet to undergo any bioactivity assays for crude or purified fractions (Harvey, 2008), thus there is vast potential for NCEs in the plant kingdom yet to be mined and explored for therapeutic activity.

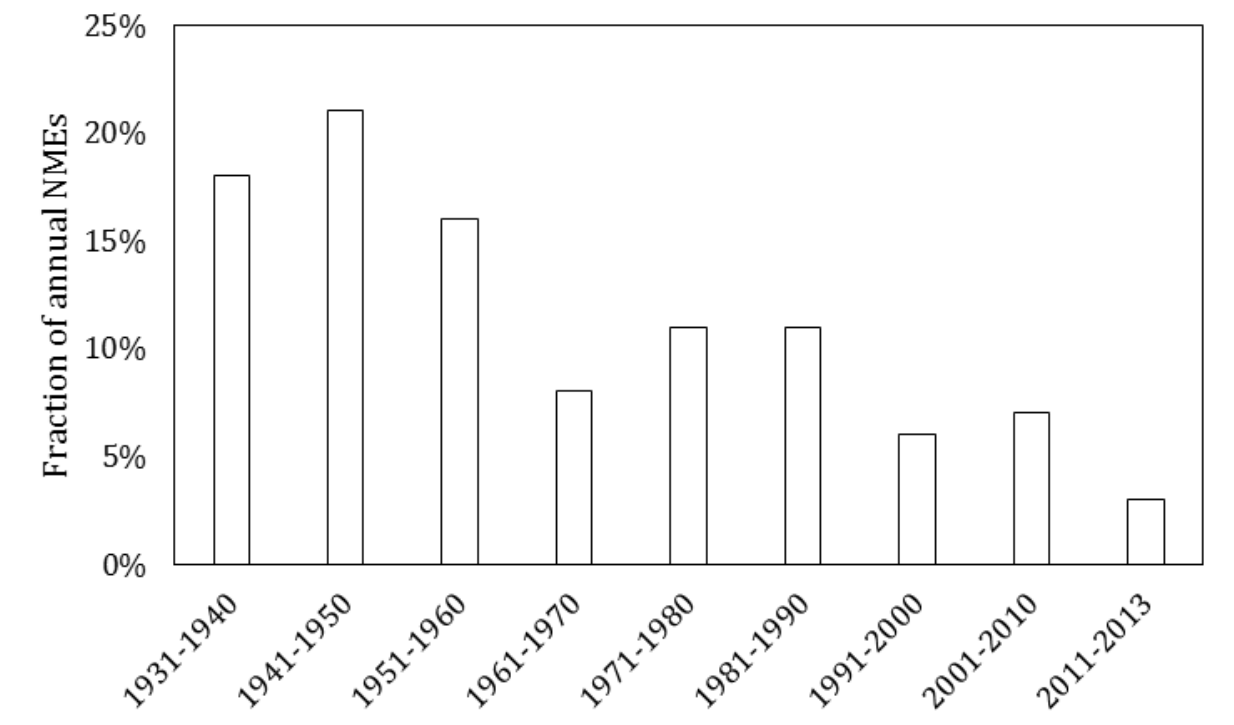

Fig 1.4: New chemical entities based or derived from plant natural products. The fraction of approved NCEs derived or based on plant natural products, from the years 1931-2013 (Adapted from Patridge et al., 2015).

\subsection{Medicinal Plants}

Plants with extensive ethnobotanical usage for medicinal purposes (medicinal plants) have higher chances of demonstrating bioactivity and producing pharmacologically significant natural products (Cragg et al., 1997; Cox et al., 1988). As such, traditional medicinal plants have proven to be a reliable subgroup of plants to be explored for bioactivity and novel structures for development of pharmaceuticals. As discussed in the previous section, digoxin, quinine and morphine were all discovered from plants with well-established ethnobotanical applications.

Whilst much controversy initially surrounded the identity of Colchicum autumnale as the medicinal plant described in ancient Egyptian papyrus documents for the treatment of rheumatism, dropsy and gout, it was positively identified in the 1830s (Hartung, 1954). The alkaloid colchicine was subsequently isolated from the bulb of the plant, and identified as the bioactive component of $C$. 
autumnale, with activity against gout attacks (Hartung, 1961). Colchicine inhibits the polymerization of tubulin and though too toxic for the treatment of cancer, it is however FDA approved for the treatment of gout and familial Mediterranean fever (Itokawa et al., 2008). It is thought to be a mild anti-inflammatory agent and affects various components of the inflammation response (Terkeltaub, 2009).

In European traditional medicine, the use of Galanthus woronowii for the treatment of poliomyelitis can be found in literature as a second-hand report (Shellard, 2000). Early work on G. woronowii and other Galanthus spp. in the 1950s was primarily carried out in Bulgaria and Russia, where galanthamine (galantamine) was first isolated (Heinrich \& Teoh, 2004). In the same decade, galanthamine was also isolated from Leucojum aestivum at higher quantities than other Galanthus spp, and its acetylcholinesterase (AChE) inhibiting and cholinesterase inhibiting activities were also determined (Heinrich \& Teoh, 2004). While galanthamine was used clinically in the 1970s for its cholinesterase inhibiting activity (Shellard, 2000), it was investigated for Alzheimer's disease (AD) in the 1980s, as a result of its ability to cross the blood-brain barrier (BBB) and to fully utilize its cholinergic activity (Heinrich \& Teoh, 2004). It was subsequently marketed in Austria in 1996 to slow down the progression of $A D$, and has since been launched in other countries such as the wider Europe (UK, Iceland, Sweden), Australia, Canada, Malaysia and the US in the early 2000s (Heinrich \& Teoh, 2004).

In 2015, the other half of the Nobel Prize for Physiology or Medicine was awarded to Youyou Tu, for her work on Artemotil ${ }^{\circledR}$, an antimalarial drug and a derivative of artemisinin (Cui \& Su, 2009; Nobel Assembly, 2015). Artemisinin was isolated from Artemisia annua, a welldocumented herb in traditional Chinese medicine (TCM) for which the dried aerial parts of which were used for the treatment of fever and malaria (Itokawa et al., 2008). Although initial tablet formulations of artemisinin as a drug proved ineffective due to poor dissemination, a capsule form of artemisinin produced better results. However the dissemination of these positive findings was limited due to the political environment in China at the time (Tu, 2011). In further molecular 
modifications of the original structure, a more stable and ten times more potent compound was synthesized, and both forms have been studied for applications to other diseases (Tu, 2011). All in all, medicinal plants have proven to be a fruitful investigative platform for the discovery of new drugs, drug leads, and NCEs.

\subsection{Samoan Medicinal Plants}

\subsubsection{History}

Samoan traditional medicine utilizing plants has relatively new beginnings, unlike Ayurvedic and traditional Chinese medicinal systems that have well-documented histories dating back to the $\sim 2000$ and $\sim 1000 B C$ respectively. Whilst the missionaries and members of US expeditions to the Samoan islands during the 1800 's had extensive periods of living with and observing Samoans, minimal reference was made to Samoan medical practices. At the time, it was noted that due to their isolation, the Samoan people did not suffer from diseases that were plaguing the Western world, such as measles, mumps, whooping cough, smallpox, tuberculosis and influenza. In contrast, Samoan people were often afflicted with diseases such as skin ulcers and infections, and several forms of eye ailments (Whistler, 1996).

During that time period, Samoan diseases were categorized into two groups: ailments with clear origins and manifestations (e.g. rashes, wounds, burns), and the other comprised ailments with internal or unclear origins (e.g. diseases often attributed to supernatural causes such as gods, spirits and ghosts) (Whistler, 1996). In the cases of rashes, wounds and burns, the extent of medical practices used to combat these included cleaning the site and wrapping with a plantain (Musa spp.) leaf (Whistler, 1996). Where ailments of unclear origins arose, Samoans attributed these to their gods, spirits and deities, and sought the assistance of a witch doctor to banish or appease the offended god, often achieved through prayers or the presentation of gifts (Harrington, 2001; Whistler, 1996). 
Surprisingly, in recent years, detailed accounts of extensive Samoan medical practices, specifically their common use of approximately 84 available plants and herbs, have been published (Harrington, 2001; Han, 1998; Whistler, 1996; Whistler, 2000). This is a contrasting shift in paradigm; whilst other Pacific islands were adopting the European medical practices, Samoans were seeking medical relief from the plants and herbs available in their natural flora (Macpherson \& Macpherson, 1990). Macpherson \& Macpherson (1990) interpreted this as a response to the new diseases and epidemics that the Europeans and missionaries brought to the islands that could neither be attributed to gods and spirits, nor successfully remedied by Europeans medications.

In preparing plant material for traditional applications, Samoan healers use various parts of the selected plant including the plant bark, roots, leaves or shoots (Harrington, 2001; Han, 1998, Whistler, 1996). These are either mashed and rubbed onto the skin or wrapped onto the wound site, or the juice squeezed from the preparation and drunk as a potion or used as an ointment (Macpherson \& Macpherson, 1990). In other instances, the plant material is mixed with coconut cream, or Samoan oil, and rubbed onto the skin or wrapped onto the affected area (Harrington, 2001; Macpherson \& Macpherson, 1990). Additionally, some traditional healers chew the plant material, and squeeze the resulting juice onto a wound, with the belief that their "mana" as a selected healer, is transferred onto the wound (Whistler, 1996). In rarer cases, a dried plant material is used as a prop in chants for the treatment of some ailments, or is burnt and the resulting smoke is fanned onto the affected site (Macpherson \& Macpherson, 1990). In the Samoan use of plants for medicinal purposes, the application is often targeted to the group of diseases mentioned earlier such as wounds, eye infections, boils, skin ulcers and infections, and supernaturally induced or hard to cure ailments of unseen origins (Whistler, 1996).

\subsubsection{Previous studies on Samoan medicinal plants}

Samoan medicinal plants as sources of bioactive compounds for drugs, drug leads and NCEs have been previously investigated. In 1973, a broad pharmacological study was undertaken on 
34 Samoan medicinal plants, with samples taken from various parts including leaves, bark, stem and roots (Norton et al., 1973). The two extracts prepared from each plant part (with polar and nonpolar solvents) were analyzed for systemic effects in rats, as well as antibacterial, antiviral and antitumor activities. Although none of the tested extracts showed significant antiviral activity, several showed antibacterial and antitumor activity, with four exhibiting general systemic toxicity. Analyses also identified strongly hypotensive activities from 19 of 34 plant species, which is a $56 \%$ hit rate for hypotensive activity alone.

Another study was carried out in 1989 in which 104 extracts from 74 Samoan medicinal plant species were investigated for pharmacological activity via an in vivo Hippocratic screen and an in vitro ileum screen (Cox et al., 1989). In this study, the Hippocratic screen was indicative of the extract effect on mice behavior which was measured by observing stimulant or depressant effects on the central nervous system, salivation and diarrhea; the ileum screen was a measure of induction or inhibition of smooth muscle contraction. This work showed that $86 \%$ of extracts inhibited ileum contraction and $41 \%$ exhibited significant effects from the Hippocratic screen while $19 \%$ of the extracts showed bioactivity in both tests.

Gustafon and associates (1992) isolated the bioactive phorbol prostratin from the Samoan medicinal plant Homalanthus nutans. Whilst prostratin was not a new chemical entity and had been previously referred to in literature, Gustafon et al. showed that unlike other phorbols, prostratin was not a tumor-promoting agent but rather a compound that exhibited strong cytoprotective activity against HIV. Medication against HIV/AIDS is limited to active HIV infection, whilst latently infected cells that act as reservoirs of the virus that exist intracellularly where drugs could not target; these reservoirs become reactive HIV infection when medication ceases (Archin \& Margolis, 2014). Extraordinarily, prostratin not only reduces infection, but it also activates HIV replication in latent cells in a mechanism involving protein kinase $\mathrm{C}(\mathrm{PKC})$, allowing the destruction of HIV from these reservoirs by other therapeutic drugs (Hezareh, 2005; Potterat \& Hamburger, 2008; Williams et al., 2004). These results supported the progression of prostratin for further evaluation and drug 
development where it is currently in Phase II clinical trials (Dias et al., 2012). Significantly, it has been hailed as a pivotal compound in the fight to eradicate HIV.

Despite the clearly promising potential of Samoan plant natural products, investigations into Samoan medicinal plants came to a standstill as the American drug discovery groups involved opted out. The potential of Samoan medicinal plants discovered in these early works (aside from prostratin) was unrealized and thus the Government of Samoa via the Scientific Research Organization of Samoa (SROS) initiated the project subject of this thesis to further explore Samoan medicinal plants and their untapped potential, more than 20 years after the last investigative endeavor.

\subsection{Chemical Genetics}

\subsubsection{Introduction}

Historically, natural products and their derivatives often entered into human use and FDA approval without a clear understanding of their mechanism of action (MOA). This has changed, and understanding drug target and drug MOA has become more commonplace and though not required for FDA approval, greatly expedites the lengthy and cumbersome process. Chemical genetics, a relatively new discipline enabled by the genomics revolution of the last two decades, has played a huge role in recent drug discovery ventures, leading to a much enhanced understanding of drug MOA and drug targets. Simply, chemical genetics is a molecular technique that combines biology and chemistry through the utilization of chemical perturbations to investigate and understand protein function and underlying molecular mechanisms (Lopez et al., 2008).

Model organisms that have been fully sequenced are another major facet of modern day drug discovery. Used with a well-defined toolbox of molecular and chemical techniques, model organisms have become powerful tools for exploratory efforts into drug target and MOA (Lopez at al., 2008). One such model, the eukaryote Saccharomyces cerevisiae (Baker's yeast) has enabled several important natural product discoveries in the recent decade. For instance, artemisinin was 
shown to mediate some of its antimalarial activity via disruption of normal mitochondrial function, elucidated through studies in yeast chemical genetics (Li et al., 2005). Furthermore, better understanding of some molecular and cellular processes has been enabled by work in yeast. For example, investigations into the target of rapamycin in yeast led to the discovery of the well conserved TOR signaling pathway (Benjamin et al., 2011).

\subsubsection{Yeast as a model organism}

S. cerevisiae yeast is a unicellular organism with $\sim 6,300$ genes that are readily amenable to genetic manipulations, a typical 90 minutes doubling time, and is very easy and cheap to culture. During the Saccharomyces Genome Deletion project, it was determined that $\sim 15 \%$ of yeast genes are "essential", and the remaining considered "non-essential" (Winzeler et al., 1999). Essential genes are those wherein the null deletion results in cell death or an inviable strain, whilst non-essential genes are those wherein the deletion allowed a viable deletion mutant. Additionally, because yeast can exist in either haploid or diploid forms, a single copy of each essential genes can be individually deleted in its diploid form, giving rise to heterozygotes which permits essential genes to be studied (Winzeler at al., 1999). The benefits of these features of yeast (gene deletions, haploid and diploid forms) will be further discussed in Section 1.5.3.1. The existence of yeast in two mating forms (MATa and MAT $\alpha$ ) permits investigations into gene-gene interactions and negative epistatic interactions, principally that of synthetic lethality (SL). SL occurs when two non-essential gene deletions individually form viable single deletion mutants, but when combined form a non-viable double mutant, which indicates the two genes are related in function (Boone et al., 2007;Tong et al., 2001). Significantly, $75 \%$ of yeast genes have high homology to human genes of similar function, with $30 \%$ of human disease genes possessing a homolog in yeast (Foury, 1997). With this high percentage of conservation between yeast and humans, much of the fundamental cellular processes and components of interest in humans are also present in yeast, making yeast an ideal model system. 


\subsubsection{Yeast Chemical Genetics}

Multiple yeast methodologies, developed following the complete sequencing of the yeast genome in 1996 (Goffeau et al., 1996), are largely adaptable to the study of bioactive compounds, in the determination of their MOA and molecular/cellular targets (Lopez et al., 2008). Much progress has been made in yeast genomics and proteomics applications. For instance, libraries of individual yeast genes tagged with fluorescent protein probes exist (e.g., GFP, RedStar, mCherry) facilitate studies into protein localization and protein expression levels in response to bioactive compounds (Bircham et al., 2011; Ghaemmaghami et al., 2003; Huh et al., 2003; Niedenthal et al., 1996). But perhaps one of the most significant developments in S. cerevisiae genomics applications was the generation of the yeast deletion mutant collection (Giaever et al., 2002; Winzeler et al., 1999).

\subsubsection{Characteristics of the gene deletion mutant collection}

The creation of the yeast deletion mutant collection was the effort of an international consortium of 15 laboratories under the Saccharomyces Genome Deletion Project. The yeast deletion collection comprises several libraries, including two haploid deletion mutant libraries of 5,000 non-essential genes, in both MATa and MATa mating types (Winzeler et al, 1999). Additionally, a diploid library of the deletions in homozygous form is also available, as well as a diploid heterozygous library of $~ 6,300$ strains, comprising both essential and non-essential genes, resulting from the deletion of a single copy of each gene. Of particular note is the feature of the library whereby each deleted gene/open reading frame (ORF) is replaced by a kanamycin resistance cassette (Fig 1.5), and a unique upstream and downstream barcode, that distinctively identifies each deleted ORF (Boone et al., 2007; Giaever et al., 2002; Winzeler et al., 1999). These libraries are, arguably, the most relevant tool for determining MOA of a bioactive compound and its molecular target, through large scale genome wide chemical genetic analyses. 


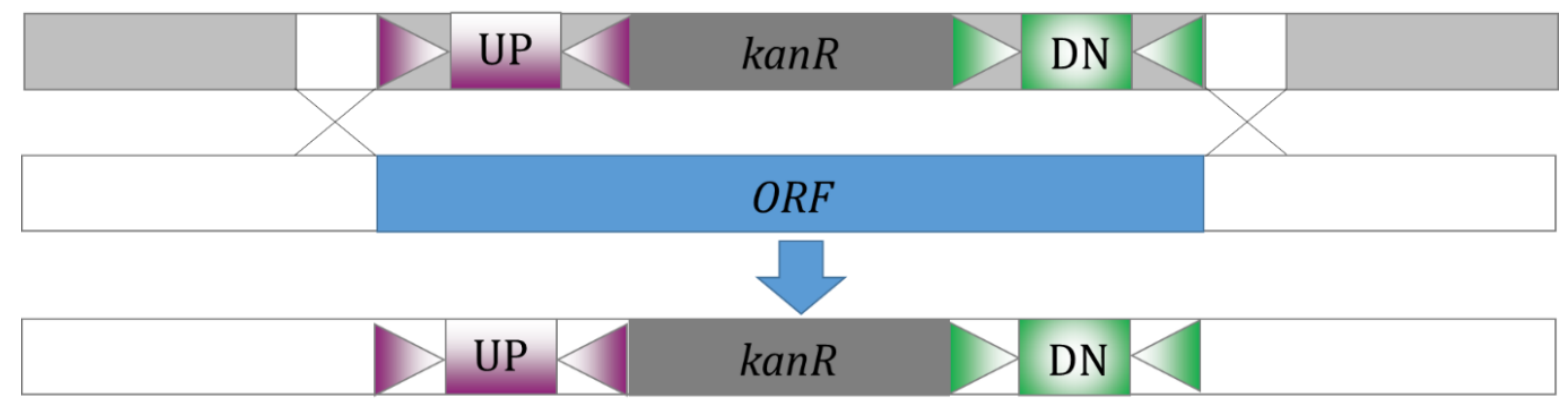

Figure 1.5: Construction strategy of the yeast deletion mutant collection. The ORF in the genomic DNA of yeast is replaced by the PCR product containing a kanamycin/G418 resistance cassette (kanR) flanked by up and down tags, via homologous recombination in yeast. The flanking tags (UP and DN) comprise 20-nucleotide molecular barcodes that uniquely identifies each ORF deletion mutant (Adapted from Boone et al., 2007).

\subsubsection{Yeast chemical genetic profiling}

Large scale genome-wide chemical genetic analysis gives rise to the identification of a chemical genetic interaction, whereby a chemical probe (e.g., a bioactive compound) results in the growth defect of a gene deletion mutant, compared to its growth in the absence of the chemical probe (Smith et al., 2010). Logically then, the utilization of the gene deletion collection for the elucidation of compound MOA is subject to a chemical probe reducing yeast cell growth. In most genome-wide analyses of a compound, several gene deletions exhibit a growth defect or hypersensitivity as a result of exposure to the chemical perturbation, and these gene deletions and the chemical form the chemical genetic profile (Fig 1.6, Boone et al., 2007; Lopez et al., 2008). This profile provides important indications to the compound MOA or compound target.

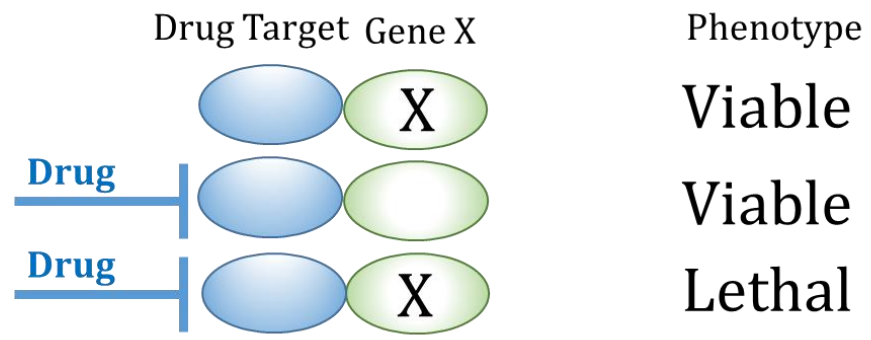

Figure 1.6: Chemical genetic interactions. The basis of a chemical genetic array whereby a gene deletion produces a viable mutant, and wild type yeast exposed to a drug treatment also produce a viable cell. However, the combination of the drug treatment and the gene deletion results in an inviable strain/lethal chemical genetic interaction (Adapted from Boone et al., 2007). 
Specifically, utilization of the heterozygous library results in a profile indicative of compound target, in what is referred to as drug-induced haploinsufficiency profiling (HIP) (Giaever et al., 1999; Smith et al., 2010). This is based on the theory that the deletion of one copy of the drug target conditions that particular heterozygote strain to hypersensitivity against the drug, and often the heterozygote strain exhibiting the highest growth defect in the presence of the drug is the drug target (Giaever et al., 1999). Conversely, the utilization of the homozygous deletion collection (homozygous profiling, HOP) or the haploid deletion collections leads to the identification of buffering mechanisms against the activity of the drug probe (Dudley et al., 2005; Fry et al., 2005; Parsons et al., 2004; Parsons et al., 2006). In the homozygous or haploid deletion mutant collections, the genetic target of a drug is completely deleted and the corresponding deletion mutant subsequently becomes resistant to the drug (Smith et al., 2010). However, the gene deletions exhibiting growth defects are identified as genes required for cell survival in the presence of the drug, suggesting that the genes function to protect and defend the cell against the effects of the drug (Hillenmeyer et al., 2010). The resulting profile from a HOP (or haploid) assay subsequently imparts information on the drug mechanism of action, based on the cell buffering mechanisms.

Importantly, given the unique barcode feature of each ORF, the chemical genetic profile of a drug can be obtained from a pooled-liquid assay where all the deletion mutants are competitively grown together; comparing the growth of treated strains to their untreated counterparts, via microarray hybridization or barcode sequencing (Bar-seq), that allows for the identification of resistant or sensitive mutants (Boone et al., 2007; Pitrowski et al., 2015; Robinson et al., 2014; Smith et al., 2012). This pooled-liquid assay format is ideal where the compound/drug of interest is of limited availability. However, cost is markedly increased (because of the expense of microarray or Bar-seq analyses), compared to a cheaper approach of screening on agar, where the chemical genetic profile is obtained when colony sizes of treated and untreated mutants are compared (Smith et al., 2010). Obtaining a chemical genetic profile utilizing the agar approach however requires larger 
quantities of the drug probe. Notably, it is important to acknowledge that conclusive demonstration of drug target and MOA require further biological characterization, and cannot simply be inferred from yeast chemical genetic profiles obtained from agar, microarray or Bar-seq analyses.

\subsubsection{Drug targets \& MOA via yeast chemical genetic profiling}

Since its invention, multiple studies have corroborated the reliability and robustness of yeast chemical genetics as a means of identifying drug target and elucidating drug MOA. Such studies employed a mixture of well characterized compounds (e.g., tunicamycin), compounds with unknown targets, as well as various growth conditions (e.g., different media, different temperature) and yeast chemical genetics profiling identified the drug targets, established buffering mechanisms as well as characterized responses to nutrient conditions. These studies were undertaken employing both HIP and HOP analyses in barcode-based pooled assays and in agar format (Baetz et al., 2004; Carroll et al., 2009; Giaever et al., 1999; Giaever et al., 2004; Hillenmeyer et al., 2008; Lum et al., 2004; Parsons et al., 2004; Pierce et al., 2007; Pitrowski et al., 2015; Robinson et al., 2014; Smith et al., 2009). Multiple studies have also utilized yeast chemical genetic approaches to identify molecular targets and MOA of natural products. These natural products include, but are not limited to, products of marine sources such as sponge-derived drugs peloruside A, neothyonidioside, laulimalide and plakortide F, as well as plant sources such as artemisinin and curcumin (Azad et al., 2013; Best et al., 2013; Li et al., 2005; Minear et al., 2011; Wilmes et al., 2012; Xu et al., 2011; Yibmantasiri et al., 2012).

\subsubsection{Yeast chemical genetic profiling of crude natural product extracts}

Yeast chemical genetic profiling has been shown to be a reliable technique in assessing MOA and biological activity of crude natural product extracts. For example, the chemical genetic profiles of two crude extracts and their respective isolated bioactive compounds were compared and it was determined that the profile of the isolated compound closely matched that of its respective 
crude extract (Parsons et al., 2006). Whilst this makes yeast chemical genetic profiling an even more important tool in natural product drug discovery by prioritizing extracts based on bioactivity, this approach is most ideal to crude extracts comprising one major bioactive compound.

\subsection{Aims}

This dissertation was based on the general question of whether Samoan medicinal plants had bioactive compounds of pharmaceutical potential, and whether their identified MOA correlate to their application in Samoan traditional medicine. Thus, the overall aim of this study was to investigate the bioactivity and mechanism of action of Samoan medicinal plants. Because yeast chemical genetics is a well-established and reliable method for such investigations, that methodology was implemented to accomplish the overall aim of this study. My attempt to address this overall aim was divided into four objectives:

1. To characterize the stability and bioactivity of aqueous and methanolic extracts of selected Samoan medicinal plants (Chapter 2).

2. To elucidate the MOA of the most potent medicinal plant by performing a haploid gene deletion-based genome-wide analysis and characterizing the implicated biological activity in order to correlate the determined MOA to its traditional application in Samoan medicine (Chapter 3).

3. To identify the target gene as well as establish both the haploinsufficiency and homozygous profiles of the extracts from the medicinal plant of interest via diploid-based genome-wide analyses (Chapter 4)

4. To identify the compound responsible for the MOA of the most potent medicinal plant (Chapter 5). 


\section{Chapter 2:}

\section{Bioactivity and Stability of Aqueous and Methanolic Extracts of Samoan Medicinal Plants}

\subsection{Introduction}

In biological studies of natural products, sourcing quantities sufficient to ensure completion of structure and mechanism of action (MOA) studies is a major issue. Over-exploitation of natural sources such as plants and animals could lead to scarcity. For instance, paclitaxel was initially sourced from the bark of the slow growing Californian coastal yeast tree, Taxus brevifolia and were it not for synthetic means of supply, T. brevifolia would have been threatened. In the interest of incurring minimal damage to Samoan plant species selected for this work, only leaves were sourced to ensure sufficient samples were collected from multiple plants per species without risking species viability. A major aim of this dissertation is to investigate the bioactivity of Samoan medicinal plants in the form that the plants are used in traditional medicine, thus pointing a focus to aqueous plant leaf extracts as this is the most common form of application. Here we selected 11 medicinal plants (Fig 2.1), and assessed their bioactivity against Baker's yeast, Saccharomyces cerevisiae. Bioactivity was assessed as the ability of the extracts to affect yeast metabolism measured as reduced yeast growth. In this work, yeast was used as the model organism primarily due to the existence and the robustness of the yeast deletion collection in identifying the genetic target or the MOA of a compound or extract.

\subsubsection{Bischofia javanica}

The leaves of B. javanica are used in Samoan traditional medicine to treat eyelid infections, blurry vision and eye injuries, where the leaf juice is dripped directly into the eyes (Whistler, 1996). The bark is used for skin sores, mouth infections and mouth sores (Harrington, 2001; Whistler, 
1996). Less commonly, the plant is also used to treat fevers, coughs, tooth-aches and gastrointestinal upsets (Uhe, 1974). In Fiji, a potion is prepared from the bark and given to children who have not walked by the time they reach two years of age; it is also used for stomach and mouth ulcers and athlete's foot (WHO, 1998). In the Solomon Islands, the stem is used to treat tuberculosis (WHO, 1998). The application of the plant to treat mouth and eye infections is common between Samoa, Tonga and Futuna Islands (WHO, 1998) and may denote some validity to the therapeutic effects of the plant. Work by Norton et al. (1974) showed that extracts from B. javanica did not display any antiviral, antimicrobial, hypotensive or antitumor activity. However, Cox and associates (1989) showed B. javanica bark extract to possess modest effects on mouse behaviour including a depressant effect on the central nervous system (CNS) and also showed activity as a muscle relaxant. In a later study, it was determined that $B$. javanica bark extract had a moderate inhibitory effect on prostaglandin synthesis (Dunstan et al., 1997).

\subsubsection{Clerodendrum inerme}

Samoan healers use the juice from crushed leaves of $C$. inerme to treat wounds, inflammation, post-partum sickness and internal injuries (Uhe, 1974; Whistler, 1996). In American Samoa, $C$. inerme is used externally in combination with other medicinal plants to treat impetigo (Harrington, 2001). The use of this plant for medicinal purposes has not been reported from other South Pacific islands. In the study by Norton and associates (1974), it was determined that whilst $C$. inerme leaf extracts had neither antiviral nor antitumor activities, the leaf extracts exhibited mild hypotensive activity as well as antimicrobial activity against Nocardia spp and Staphylococcus aureus. It was later determined to also have modest muscle relaxant activity (Cox et al., 1989).

\subsubsection{Colubrina asiatica}

Whistler (1996) reported only a single use for this plant in Samoan medicine. The leaves, either boiled or fresh, are used to prepare a potion taken to treat postpartum sickness. In American 
Samoa, more extensive use of the plant in traditional medicine is reported to include treatment of gonorrhoea, abscesses and a Samoan ailment literally translated to spreading inflammation rash; this ailment often results in death if not treated immediately and is postulated to be the result of septicaemia (Harrington, 2001). Methanol and ethanol extracts of $C$. asiatica leaves exhibited antiviral, antitumor and strong hypotensive activities although no antimicrobial activity was detected (Norton et al., 1974). When applied in vivo, C. asiatica extracts caused death in mice, while in vitro studies showed a significant muscle relaxant activity (Cox et al., 1989).

\subsubsection{Cordyline fruticosa}

The leaves of $C$. fruticosa are widely used by Samoan healers for massages (Whistler, 1996). In American Samoa, it is taken internally in combination with other plants for the treatment of intestinal upset in children that have the specific symptoms of loose greenish stool and difficulty in defecating (Harrington, 2001). Across the Pacific, the various plant parts are used to treat lower chest pains, filariasis, postpartum sickness, ear ache, eye infections, colds, coughs, eczema, laryngitis, gum abscesses, inflammations, aching limbs and fevers (WHO, 1998). In addition, Cox et al. (1989) reported a significant muscle relaxant activity from C. fruticosa leaf extracts.

\subsubsection{Cymbobogon citratus}

C. citratus is used by both Samoan healers and lay people to treat ailments involving children, particularly mouth sores and mouth infections. The leaves are crushed or chewed, and the juice dripped onto the mouth sores or taken as a potion (Whistler, 1996). This application is also observed in American Samoa and Tonga (Harrington, 2001; Whistler, 1996). Less commonly, it is use to treat filariasis (Uhe, 1974). A significant muscle relaxant activity and mild central nervous system depressant (CNS) activity has been reported from a stem extract of $C$. citratus (Cox et al., 1989). 


\subsubsection{Flacourtia rukam}

Whistler (1996) reported the application of the F. rukam bark for the treatment of inflammation, and its leaves in the treatment of sores in Samoan traditional medicine. McCuddin (2001) reported the use of the inner bark of F. rukam in combination with other plants in a potion taken for the treatment of an ailment exhibiting specific symptoms, including soreness and difficulty in opening eyes, the feeling of having a swollen head, cold sweat, dizziness, and leg numbness. Extracts from F. rukam did not demonstrate any antiviral, antitumor or antibacterial activities; however, the ethanol extract from its leaves proved hypotensive (Norton et al., 1974). Further, extracts from the stem and bark exhibited mild to moderate CNS depressant and muscle relaxant activities (Cox et al., 1989).

\subsubsection{Macropiper puberulum}

Potions made from the leaves of M. puberulum are used to treat diseases believed to be of ghost or supernatural origins in Samoan traditional medicine (Whistler, 1996). In American Samoa, its use has been reported for the treatment of cellulitis and various other forms of inflammation (McCudden, 2001). The leaves and stems have also been used to treat influenza, convulsions, swellings of the testicles, swollen breasts, toothache and scabies in Fiji, whilst in Tonga, $M$. puberulum leaves are used to treat inflammation and boils (WHO, 1998). Although no scientific analyses have been done of $M$. puberulum, other members of the Piperaceae family, such as Piper graeffi and $P$. methysticum, have been shown to have mild CNS depressant activity, a significant muscle relaxant activity and moderate inhibitory effects on prostaglandin biosynthesis (Cox et al., 1989; Dunstan et al., 1997).

\subsubsection{Psychotria insularum}

One of the most commonly used medicinal plants in Samoa against ailments attributed to ghosts and spirits (supernatural sources) is $P$. insularum, wherein the leaves or bark are crushed and 
used to make a potion (Whistler, 1996). The leaves are also used to treat ailments such as burning fever, abdominal distress, abscess, incontinence, and scrotal swelling in elephantiasis (Cox et al., 1989). Additionally, crushed leaves of the plant are applied directly to skin infections or wounds, and the plant is also used for the treatment of general body aches and swellings (Whistler, 1996). In American Samoa, $P$. insularum is often taken in combination with other medicinal plants for the treatment of vomiting or coughing up blood (unrelated to tuberculosis or gastrointestinal upset) and cellulitis (Harrington, 2001). P. insularum is not used for medicinal purposes elsewhere. Norton and associates (1974) detected mild hypotensive activity from extracts of $P$. insularum, although no antiviral, antitumor or antimicrobial activity was identified. In another study, P. insularum severely reduced motor function in mice, and proved toxic at high concentrations producing death in mice (Cox et al., 1989). An anti-inflammatory effect of $P$. insularum extract has also been reported, exhibiting a strong inhibitory effect on both prostaglandin biosynthesis and rat ear oedema (Dunstan et al., 1997).

\subsubsection{Syzygium corynocarpum}

Samoan traditional medicine makes use of $S$. corynocarpum leaves for the treatment of skin sores as well as urinary tract problems (Whistler, 1996). Less commonly, it is used for the treatment of tuberculosis, sore throats, wounds and high fever (Uhe, 1974). McCuddin (2001) has recorded its use for erythraemia and fever in American Samoan traditional medicine. Leaf extract of S. corynocarpum caused death in mice and produced a strong muscle relaxant activity (Cox et al., 1989). Mild inhibition of prostaglandin and rat ear oedema was detected by Dunstan and associates (1997).

\subsubsection{Vigna marina}

Potions prepared from crushed $V$. marina leaves are used to treat infants that present with various ailments whereby the leaf juice is taken as a potion dripped into the ears, nose, mouth and 
eyes, or alternatively rubbed onto the skin (Whistler, 1996). American Samoan traditional medicine uses $V$. marina in combination with other plants for the treatment of cellulitis and supernaturally induced ailments whereby the patient cannot sleep and is very talkative all day and night (Harrington, 2001). Leaf extracts of $V$. marina have been shown to exhibit mild muscle relaxant activity (Cox et al., 1989).

\subsubsection{Wollastonia biflora}

Whistler (1996) reported the most common application of this plant is for the treatment of urinary tract problems, for which the juice from the leaves of the plant is taken as a potion. Sometimes, the juice is taken to treat hepatitis and stomach ache (Whistler, 1996). In addition to the above applications, American Samoan healers also use W. biflora to treat gonorrhoea (Harrington, 2001). Leaf extracts of $W$. biflora did not exhibit antiviral, antitumor or antimicrobial activity, but did exhibit mild hypotensive activity (Norton et al., 1974). In an in vivo study, administration of $W$. biflora leaf extracts at high concentrations proved toxic in mice, while in vitro studies showed the extracts exhibited strong muscle relaxant activity and mild inhibitory effects on prostaglandin biosynthesis (Cox et al., 1989; Dunstan et al., 1997).

\subsubsection{Aim}

The aim of this chapter was to investigate and determine the bioactivity of the selected medicinal plants, establish their amenability to genome-wide analyses in yeast as a first step in determination of MOA, and to identify the medicinal plant producing the most potent extracts. This was achieved through monitoring the growth profiles of yeast in the presence of aqueous and methanolic extracts from the above 11 medicinal plants. 

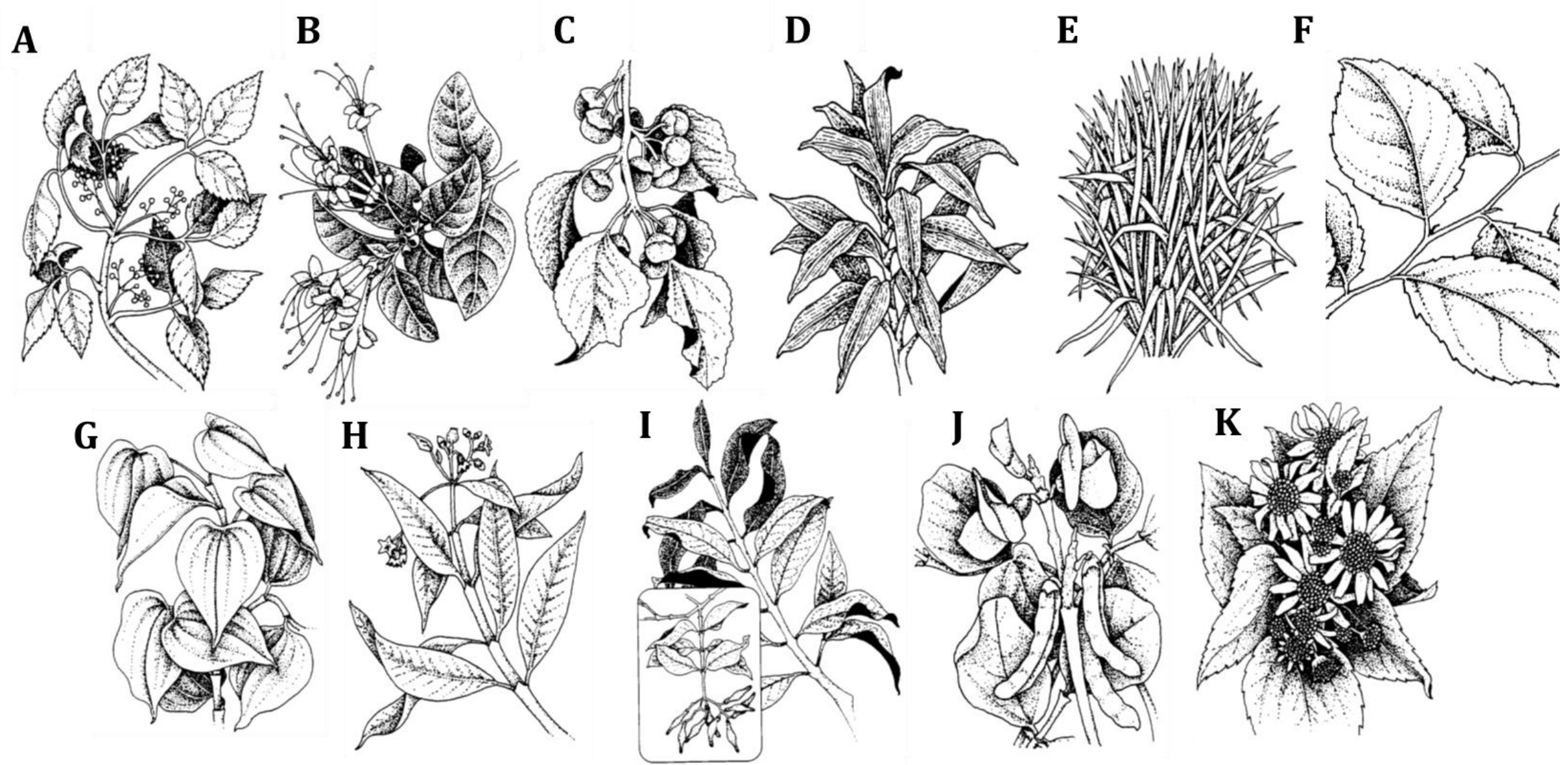

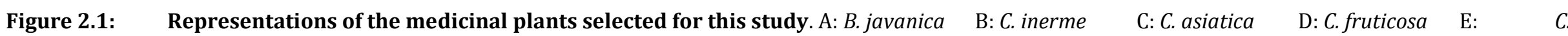




\subsection{Methods}

\subsubsection{Medicinal plant collection}

Permission was sought and obtained from the Ministry of Natural Resources and Environment (MNRE) Samoa in agreement with the Convention of Biological Diversity stipulations for the collection and export of Samoan medicinal plant material. Approval was also sought and acquired from the Ministry of Primary Industries (MPI NZ) for the importation of the plant material into New Zealand for research purposes. Sample identification and collection was carried out with the assistance of the Environment and Conservation Division within MNRE, specifically the Principal National Parks officer Mr Talie Foliga who worked extensively with Dr Arthur Whistler, the scientist that documented the extensive ethnobotany of Samoa (Atherton \& Jefferies, 2012; Whistler, 1996; Whistler, 2000). Upon positive plant identification, leaves were collected from national parks and reserves managed by MNRE, as well as some private properties. Because a comparison of plant populations was not a factor in this study, leaves were collected from plants in one location (Fig 2.2). Once the leaves were washed, quantity required (weight was not measured) to produce $1 \mathrm{~L}$ of juice was juiced using an industrial juicer (Breville Juicer BJE410). The collected juice was stored at $4^{\circ} \mathrm{C}$ and shipped on ice to the Chemical Genetics Laboratory at Victoria University of Wellington.

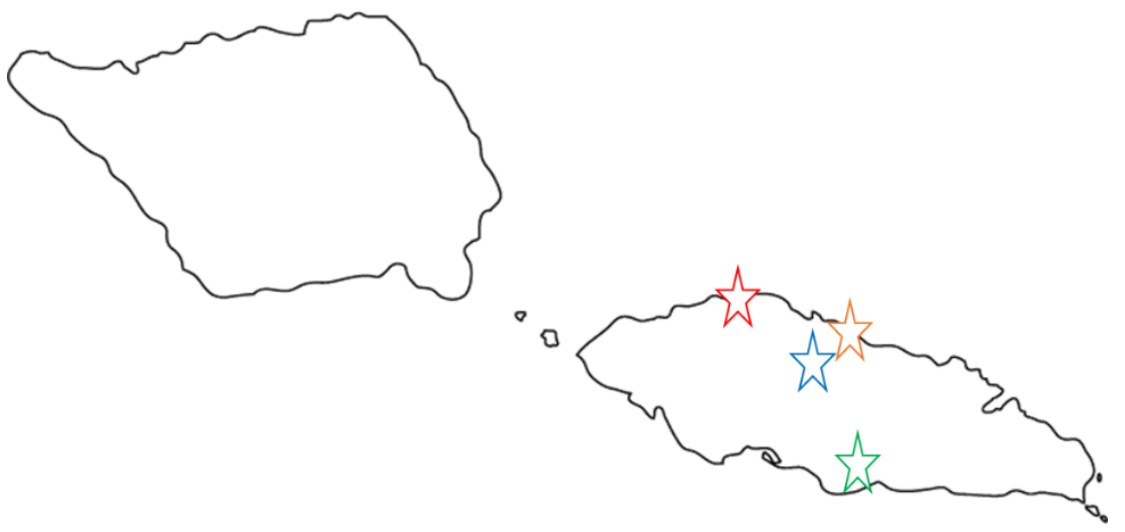

Figure 2.2: $\quad$ Location of collection of Samoan medicinal plants used in this study. The red star indicates village of Saleimoa, where B. javanica, C. inerme, C. asiatica, P. insularum, S. corynocarpum, V. marina and $W$. biflora were collected from various private properties. The orange star marks Vaivase from where $C$. corynocarpum was collected from a single private property. The blue star marks the Vailima Botanical Gardens, where F. rukam was collected from. The green star marks the Pupu-Pue National Park, which was the collection site of C. fruticosa and M. puberulum. 


\subsubsection{Extract preparation}

Because this work focused on studying the plant extracts in the form used in traditional Samoan medicine, the juices from fresh leaves were prepared for biological analyses. As the yeast liquid-based growth assays were quantified via absorbance (spectrophotometric) measurements, the solid material from each leaf juice sample was removed by centrifugation at $10,000 \times \mathrm{g}$ for 30 min. The supernatant was collected and sequentially filtered through $7 \mu \mathrm{m}, 1.2 \mu \mathrm{m}, 0.45 \mu \mathrm{m}$ and $0.22 \mu \mathrm{m}$ filters to remove any solid particles and to ensure sterility. The resultant solutions were labelled aqueous extracts and stored at $-20^{\circ} \mathrm{C}$ in $1 \mathrm{~mL}$ aliquots.

The solid material removed from centrifugation was extracted in methanol at a ratio of $1 \mathrm{~g}$ solid plant material to $9 \mathrm{~mL}$ methanol; extraction was carried out at $30^{\circ} \mathrm{C}$ whilst shaking overnight. The solution was centrifuged at $10,000 \times \mathrm{g}$ for $30 \mathrm{~min}$, and the supernatant was collected and filtered through $0.22 \mu \mathrm{m}$ filters to ensure sterility. Methanol was removed using a cold trap system (Labconco Centrivap), and the solid methanol extract was resuspended in DMSO (1 $\mathrm{mL}$ methanol extraction mass to $200 \mu \mathrm{L}$ DMSO). Aliquots were stored at $-20^{\circ} \mathrm{C}$. The aqueous and methanol extractions are summarized in Fig 2.3.

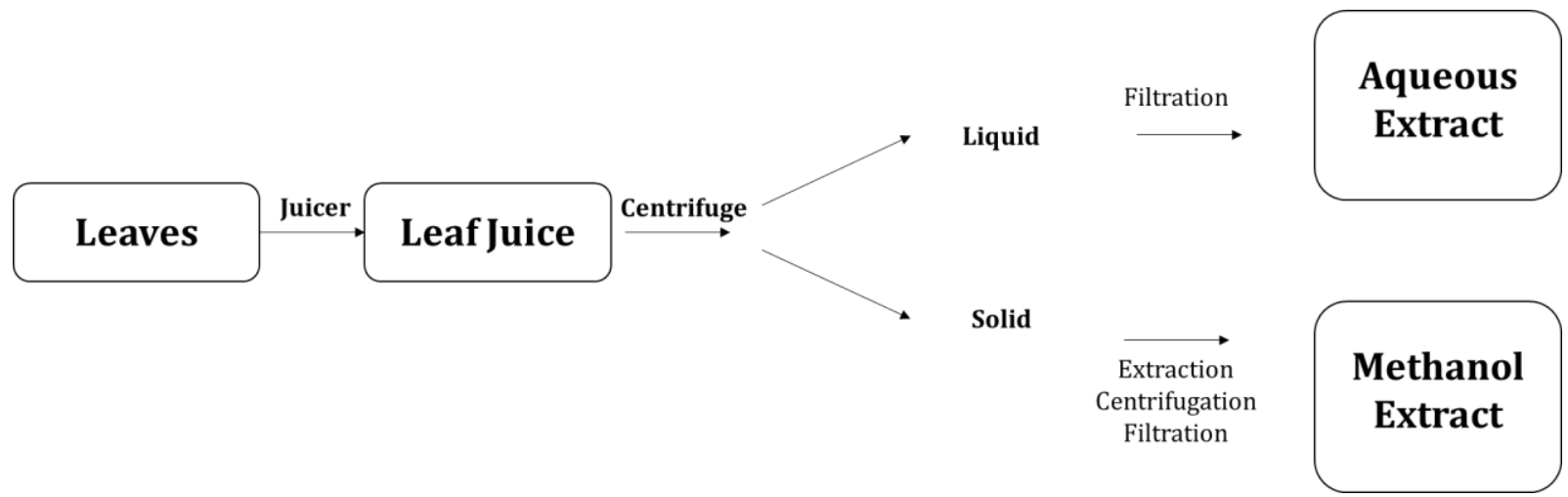

Fig 2.3: Schematic of leaf juice sample preparation for analyses. Leaves were collected, washed and homogenized to obtain leaf juice, which was then centrifuged to separate the liquid from the solid part. The liquid was filtered to generate the aqueous extracts. The solid part was extracted in methanol overnight, then centrifuged to remove the solid fraction, and the supernatant was filtered. The methanol was removed and the resulting dried methanol extract mass was resuspended in DMSO. 


\subsubsection{Liquid-based bioactivity assays}

Determination of extract bioactivity was achieved by monitoring the growth of yeast as previously described (Amberg et al., 2005) with the specification that the wild-type (WT) yeast strain BY4741 was quantified in the presence and absence of the extracts. Briefly, BY4741 that was streaked on a Yeast Peptone Dextrose (YPD - Appendix I) agar plate was used to inoculate $5 \mathrm{~mL}$ YPD and incubated at $30^{\circ} \mathrm{C}$ in a rotating drum overnight for $15-18 \mathrm{~h}$. Optical density (OD) was determined at $660 \mathrm{~nm}$ using a UNICAM 8625 UV/VIS spectrometer and cell density was subsequently calculated using the OD to cell count conversion, before the cells were washed with water to remove YPD. Cells were resuspended in $\mathrm{dH}_{2} \mathrm{O}$ and used to inoculate $10 \mathrm{~mL}$ of Synthetic Complete (SC) media at $5 \times 10^{5}$ cells $/ \mathrm{mL}$. The experimental suspension was then set up to a final volume of $100 \mu \mathrm{L}$ by adding the relevant volume of extract to the relevant volume of cell suspension (e.g., $99 \mu \mathrm{L}$ cell suspension $+1 \mu \mathrm{L}$ of extract, or $95 \mu \mathrm{L}$ cell suspension $+5 \mu \mathrm{L}$ of extract). The plate was shaken at $1000 \mathrm{rpm}$ for $30 \mathrm{~s}$ using a MixMate plate shaker (Eppendorf), before absorbance was measured at $590 \mathrm{~nm}$ for t0 using the Envision 2102 Multilabel plate reader (Perkin Elmer). After $15 \mathrm{~h}$ of incubation at $30^{\circ} \mathrm{C}$, the plate was shaken at $1,000 \mathrm{rpm}$ for $30 \mathrm{~s}$ before the absorbance was read at $590 \mathrm{~nm}$ for t15. Experiments were carried out with three biological replicates, and each biological replicate had three technical replicates. Residual growth of yeast was calculated by normalizing absorbance (to remove any colour-associated absorbance interference due to the extract) by subtracting the absorbance of t0 from t15 (n-Abs). The absorbance of each individual extract treatment was then divided by the normalized absorbance of the vehicle control, multiplied by 100 . Because the yeast system was used, the residual growth determined to be cut-off point for determination of bioactivity was $80 \%$, as $20 \%$ growth reduction allowed for sufficient growth inhibition to identify buffering mechanisms, but not too high that a general cell-defence response is

$$
\text { RG (\%) }=\frac{\text { Treatment (n-Abs) }}{\text { Control }(\mathrm{n}-\mathrm{Abs})} \quad \mathrm{x} \quad 100
$$

detected. 


\subsubsection{Agar-based bioactivity assays}

Relevant volumes of extracts and controls for the relevant concentrations tested were aliquoted separately into wells of a 24 -well plate. Then $1 \mathrm{~mL}$ molten agar $\left(\sim 50^{\circ} \mathrm{C}\right)$ was added to each well, gently mixed by pipetting, and allowed to set at room temperature (RT) for $2 \mathrm{~h}$. Once set, $2 \mu \mathrm{L}$ of diluted BY4741 WT cells were spotted onto each well three times. Spots were allowed to dry before plates were inverted and incubated at $30^{\circ} \mathrm{C}$ for $24 \mathrm{~h}$. The plates were photographed using a Canon EOS 600D camera, then re-incubated for an additional $24 \mathrm{~h}$, photographed again, and visually analysed for growth defect in extract treatments compared to their relevant control. 


\subsection{Results}

\subsubsection{Two of 11 aqueous extracts are bioactive at $\mathrm{pH} 4.5$}

To determine if extracts reduced yeast growth in liquid, the growth of BY4741 WT yeast in the presence of the plant extracts was investigated. A defined quantity of cells ( $5 \times 10^{5}$ cells $\left./ \mathrm{mL}\right)$ was inoculated into $\mathrm{SC}$ media at $\mathrm{pH} 4.5$ containing either the vehicle control or a range of concentrations of the aqueous extracts. Absorbance was measured before (t0) and after $15 \mathrm{~h}$ (t15) of growth at $30^{\circ} \mathrm{C}$. The highest tested concentration of aqueous extract was $5 \% \mathrm{v} / \mathrm{v}$, with 1 in 5 serial dilutions to the lowest tested concentration of $3.2 \times 10^{-4} \% \mathrm{v} / \mathrm{v}$.

The residual growth of BY4741 in the presence of the 11 tested aqueous extracts was calculated (Fig 2.4). Residual growth of BY4741 in most aqueous extracts remained around $100 \%$ despite increasing extract concentrations. Residual growth of $100 \%$ reflects growth of extract treated cells relative to control cells, thus indicative of no growth inhibition and no bioactivity. This lack of bioactivity was observed from the aqueous extracts of $C$. inerme, $C$. asiatica, $C$. fruticosa, $C$. citratus, F. rukam, M. puberulum, S. corynocarpum, V. marina and W. biflora. Contrastingly, two growth profiles exhibited residual growth of $80 \%$, indicative of growth inhibition greater than $20 \%$. These aqueous extracts were isolated from $F$. rukam and $P$. insularum. These findings are positive regarding bioactivity of Samoan medicinal plants, with two from 11 aqueous extracts producing bioactivity in yeast at $\mathrm{pH} 4.5$. 

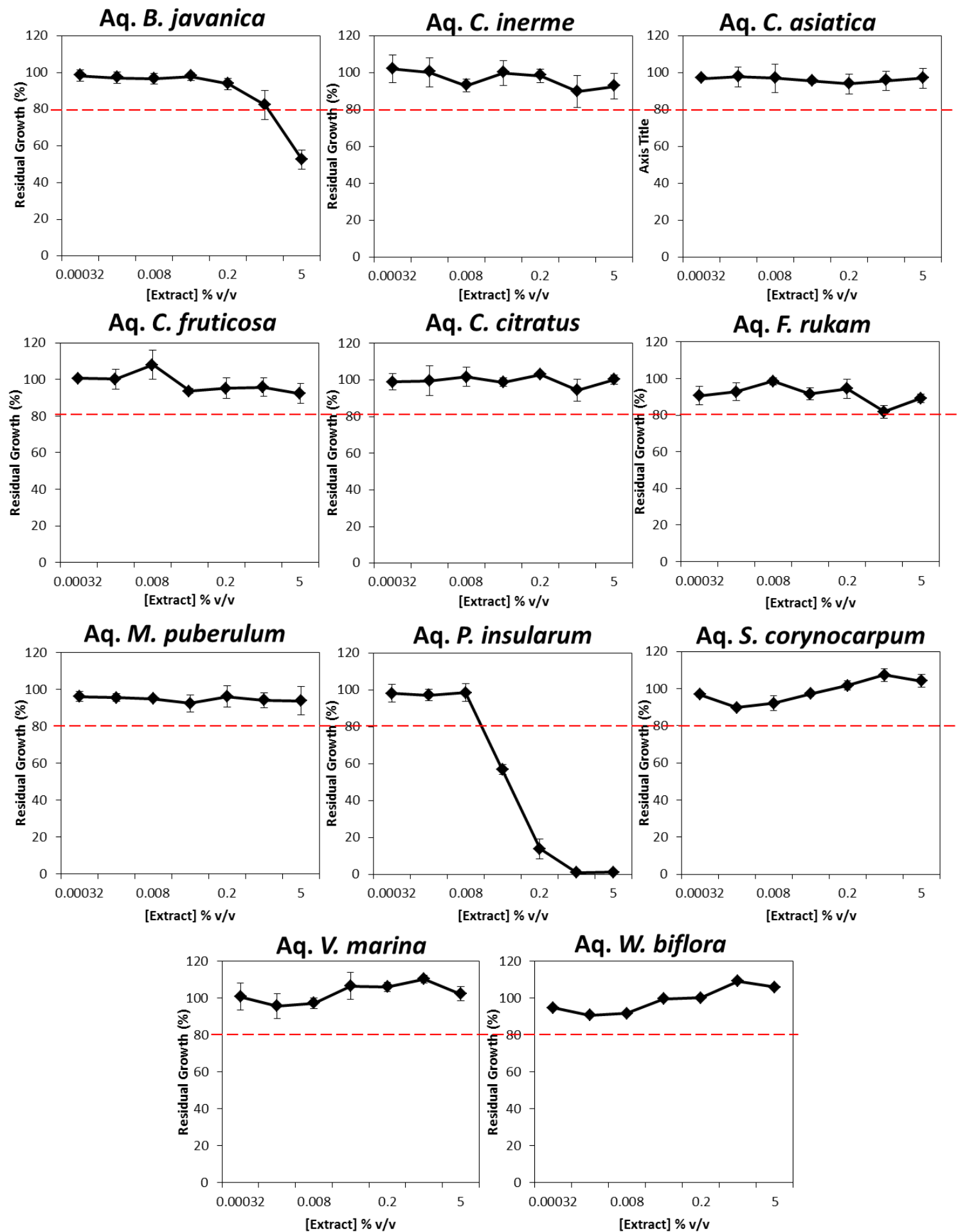

Figure 2.4: $\quad$ Liquid-based bioactivity assays of aqueous plant extracts in SC at pH 4.5. Residual growth of BY4741 (WT) in the presence of the 11 aqueous extracts of Samoan medicinal plants. BY4741 cells inoculated into SC were treated with increasing concentrations of aqueous extracts from $3.2 \times 10^{-4}$ to $5 \% \mathrm{v} / \mathrm{v}$, and growth was compared against vehicle control grown cells (SC + water). Red dashed lines mark threshold for indication of bioactivity (i.e. residual growth decreasing lower than $80 \%$ and exhibiting greater than $20 \%$ growth inhibition). 


\subsubsection{Five of 11 aqueous extracts are bioactive at $\mathrm{pH} 7.0$}

Various factors can affect extract bioactivity, $\mathrm{pH}$ being a major one. Given that the leaf extracts were often taken as potions in their application in traditional Samoan medicine, it was postulated that assessment of bioactivity may be enhanced if analyses were carried out at a $\mathrm{pH}$ closer to physiological $\mathrm{pH}$. To determine if $\mathrm{pH}$ affected bioactivity of aqueous extracts, cells were treated similarly to the previous experiment, except the SC media used was buffered to $\mathrm{pH} 7.0$ with 25 mM HEPES buffer.

The residual growth of BY4741 showed varied growth profiles at pH 7.0 across the 11 aqueous extracts (Fig 2.5). Aqueous extracts from six plants produced growth profiles whereby residual growth was maintained at control levels (100\%); these extracts were obtained from $C$. asiatica, C. fruticosa, C. citratus, F. rukam, S. corynocarpum, and V. marina. Conversely, aqueous extracts from five plants (B. javanica, C. inerme, M. puberulum, P. insularum and W. biflora) produced growth profiles whereby residual growth progressed lower than $80 \%$ indicative of bioactivity. These latter aqueous extracts were thus bioactive in $\mathrm{SC}$ media buffered to $\mathrm{pH} 7.0$ with HEPES, providing an enhanced bioactive profile compared to that obtained in $\mathrm{SC}$ media at $\mathrm{pH} 4.5$. Therefore, experimental studies conducted with aqueous extracts were carried out in $\mathrm{SC}$ pH 7.0 from this point forth. 
Aq. B. javanica

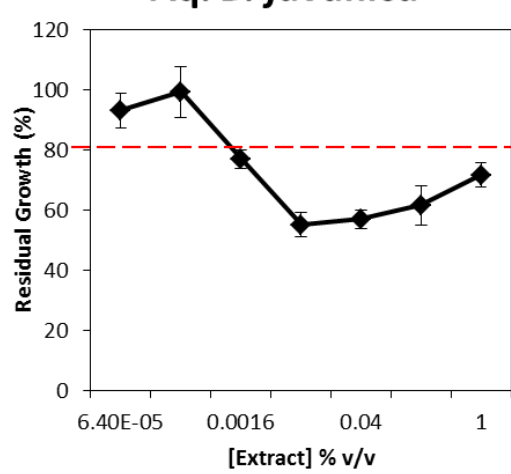

Aq. C. fruticosa

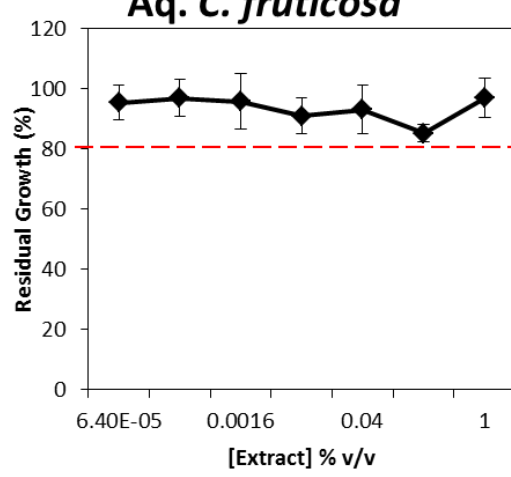

Aq. F. rukam

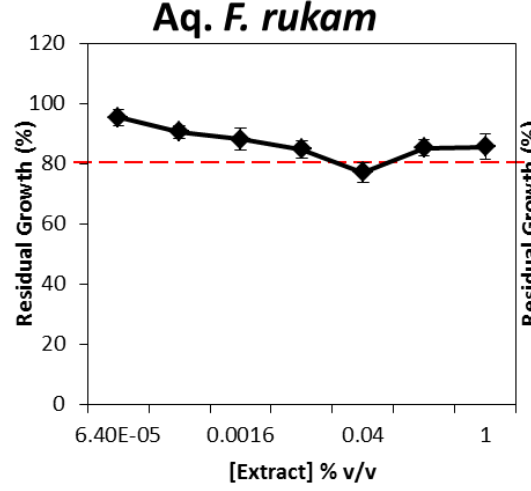

Aq. V. marina
Aq. C. inerme

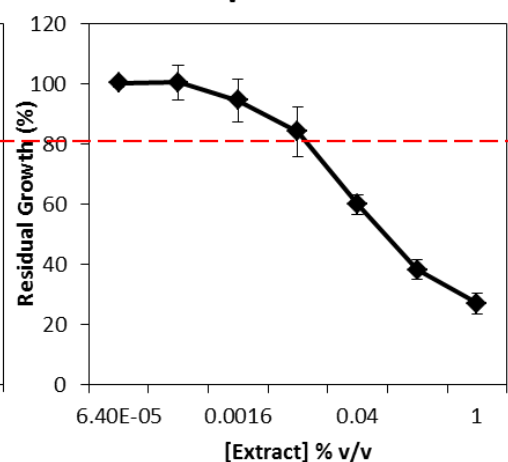

Aq. C. citratus

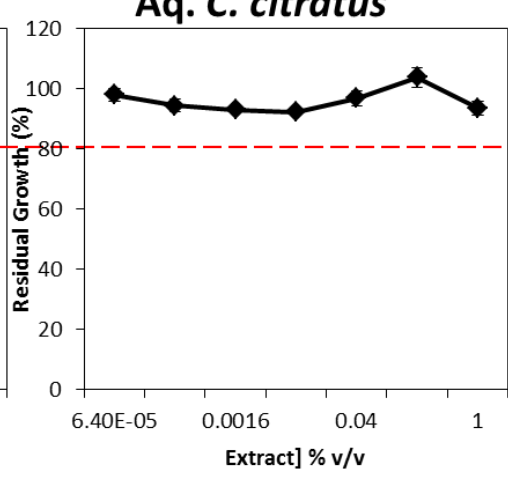

Aq. P. insularum

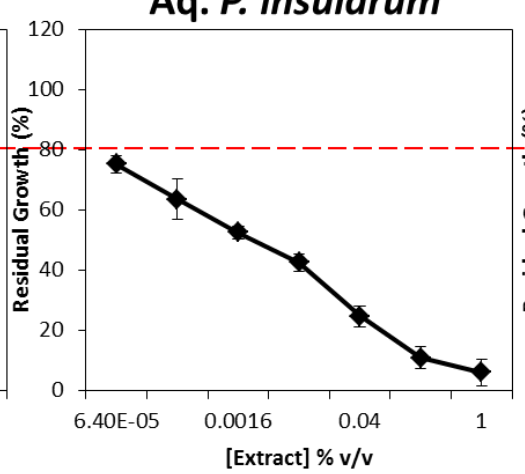

Aq. C. asiatica

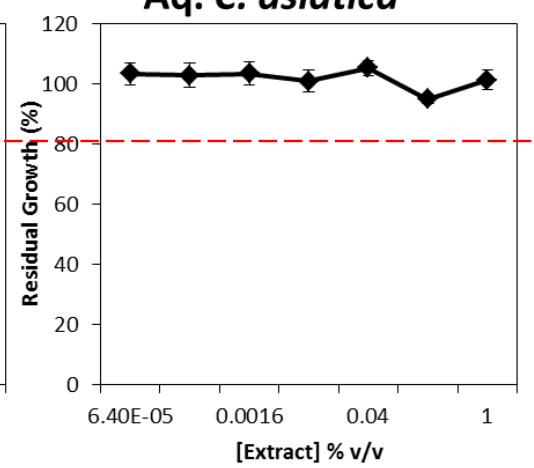

Aq. M. puberulum
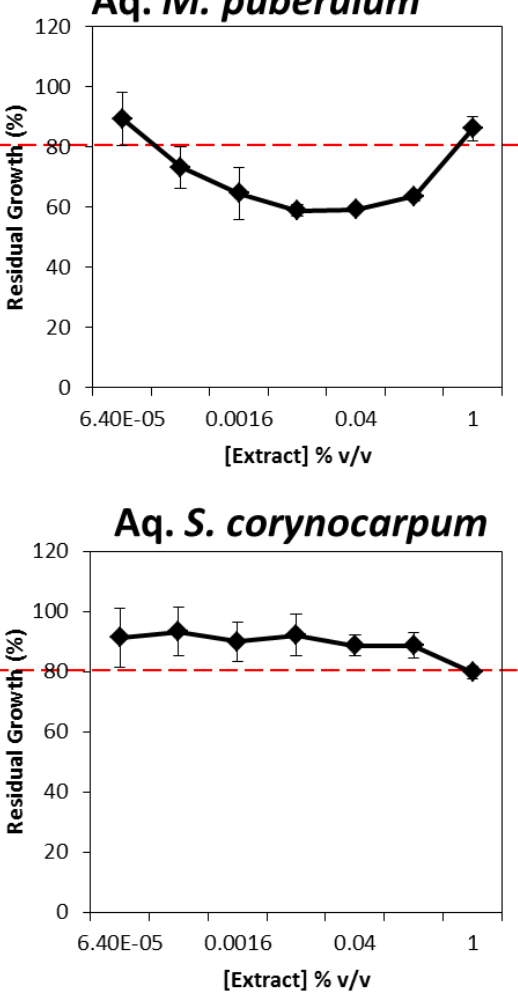

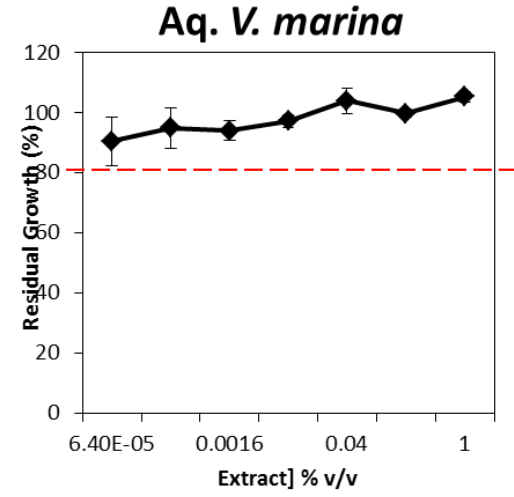

Aq. W. biflora

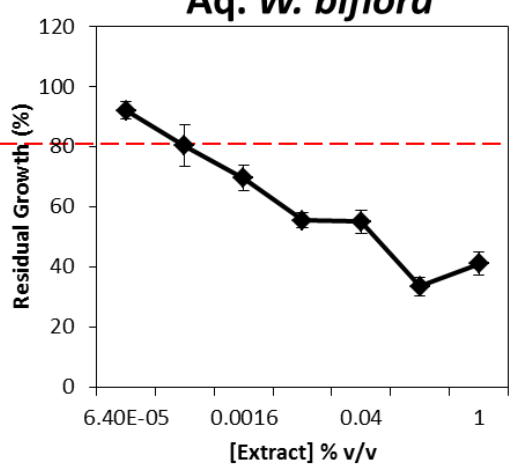

Figure 2.5: Liquid-based bioactivity assay of aqueous extracts in pH 7.0 SC. Residual growth assays of the 11 aqueous extracts of Samoan medicinal plants; cells inoculated into SC buffered to $\mathrm{pH} 7.0$ with $25 \mathrm{mM}$ HEPES $(\mathrm{SCH})$, were treated with increasing concentrations of aqueous extracts from $3.2 \times 10^{-4}$ to $5 \% \mathrm{v} / \mathrm{v}$, and growth was compared against control cells. Red dashed lines mark threshold for indication of bioactivity (i.e., residual growth decreasing lower than $80 \%$ and exhibiting greater than $20 \%$ growth inhibition). 


\subsubsection{Five of 11 methanolic extracts are bioactive at $\mathrm{pH} 4.5$}

To assess the bioactivity of the methanolic plant extracts, BY4741 was grown in the presence of increasing concentrations of methanolic extracts in SC media at pH 4.5. Concentrations tested for methanolic extracts ranged from $6.4 \times 10^{-5}$ to $1 \% \mathrm{v} / \mathrm{v}$ at five-fold serial dilutions. The maximum concentration tested was $1 \% \mathrm{v} / \mathrm{v}$ as it is established that DMSO at higher concentrations can affect the growth profile of yeast (Sadowska-Bartosz et al., 2013).

In line with the results from the aqueous extracts, the residual growth of BY4741 in the presence of methanolic extracts was recovered compared to growth in vehicle control $(1 \% \mathrm{v} / \mathrm{v}$ DMSO) (Fig 2.6). Whilst BY4741 showed a growth profile around $100 \%$ in the presence of some extracts, it also showed a decreasing growth profile in the presence of other extracts. Specifically, six methanol plant extracts produced BY4741 growth profiles that remained between 80 and $100 \%$ residual growth. These methanol extracts were from B. javanica, C. fruticosa, C. citratus, F. rukam, V. marina and W. biflora. The remaining five extracts produced BY4741 growth profiles that declined past the $80 \%$ residual growth line. These extracts were from $C$. inerme, $C$. asiatica, $M$. puberulum, $P$. insularum and S. corynocarpum. Interestingly, P. insularum, growth was nearly completely inhibited at $1 \% \mathrm{v} / \mathrm{v}$ of these extracts. 

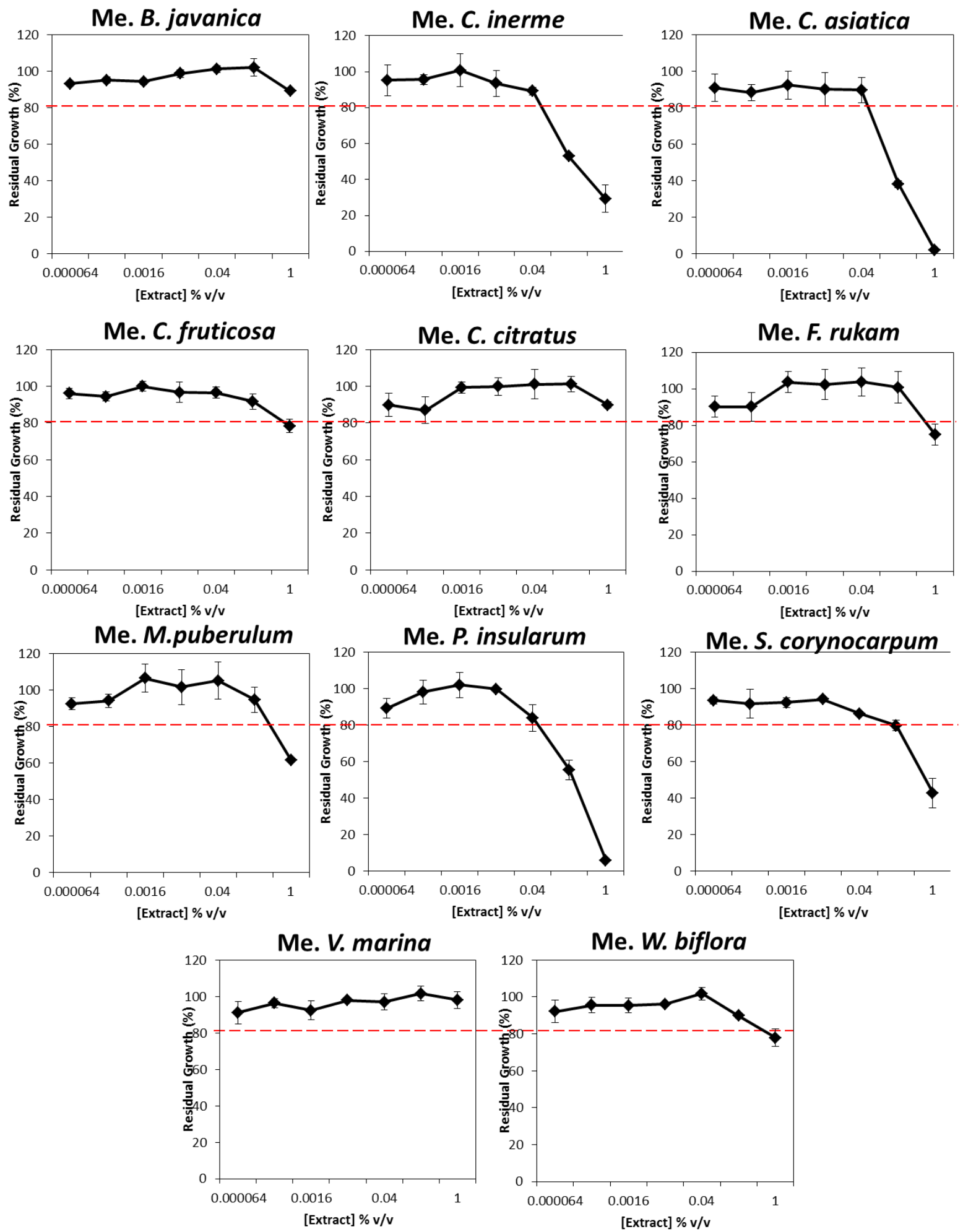

Figure 2.6: Liquid-based bioactivity assay of methanol extracts in pH 4.5 SC. Residual growth assays of the 11 methanol extracts of Samoan medicinal plants. BY4741 (WT) inoculated into SC were treated with increasing concentrations of methanol extracts from $6.4 \times 10^{-5}$ to $1 \% \mathrm{v} / \mathrm{v}$, and growth was compared against DMSO control cells. Red dashed lines mark threshold for indication of bioactivity (i.e., residual growth decreasing lower than $80 \%$ and exhibiting greater than $20 \%$ growth inhibition). 


\subsubsection{Ten of 11 methanolic extracts are bioactive at $\mathrm{pH} 7.0$}

Since $\mathrm{pH}$ was shown to play a significant role in the bioactivity of aqueous extracts of Samoan medicinal plants (Fig 2.4), we investigated if this was the same for the methanolic extracts. The previous experiment at $\mathrm{pH} 4.5$ was repeated in SC media buffered to $\mathrm{pH} 7.0$ with $25 \mathrm{mM}$ HEPES. Residual growth of BY4741 was then measured and compared to growth in DMSO control (Fig 2.7). Impressively, 10 out of 11 methanol extracts reduced BY4741 growth past the $80 \%$ residual growth line; these methanolic extracts were from B. javanica, C. inerme, C. asiatica, C. fruticosa, F. rukam, M. puberulum, P. insularum, S. corynocarpum, V. marina and W. biflora. In these profiles, B. javanica extract consistently produced a BY4741 growth profile whereby growth decreases, but before it reaches complete or close to complete inhibition, growth improves again. Extracts that reduced BY4741 growth significantly, but did not reach the point of complete inhibition at $1 \% \mathrm{v} / \mathrm{v}$ include $C$. inerme, M. puberulum and S. corynocarpum. Methanolic extracts from $C$. asiatica and $P$. insularum showed profiles that reached close to $0 \%$ residual growth by $1 \% \mathrm{v} / \mathrm{v}$. The $P$. insularum extract significantly reduced growth close to complete inhibition at $0.008 \% \mathrm{v} / \mathrm{v}$, the lowest concentration producing close to complete inhibition observed from either aqueous or methanol extracts. 

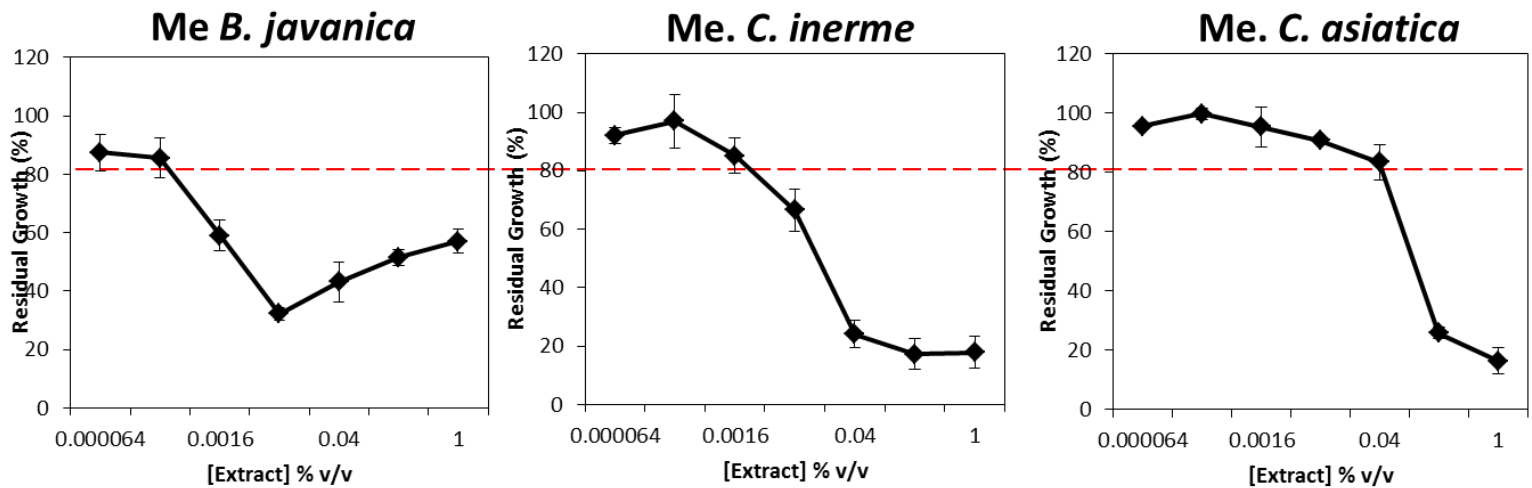

Me. C. fruticosa

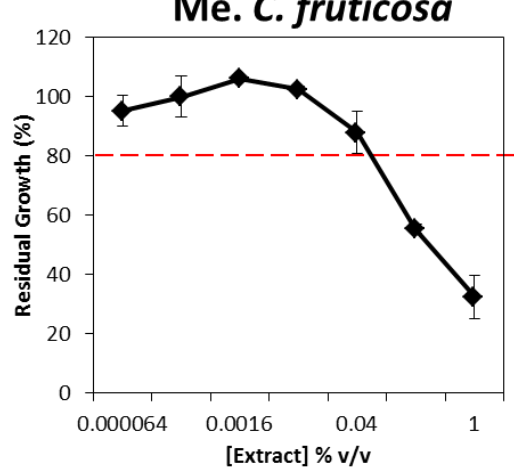

Me. C. citratus
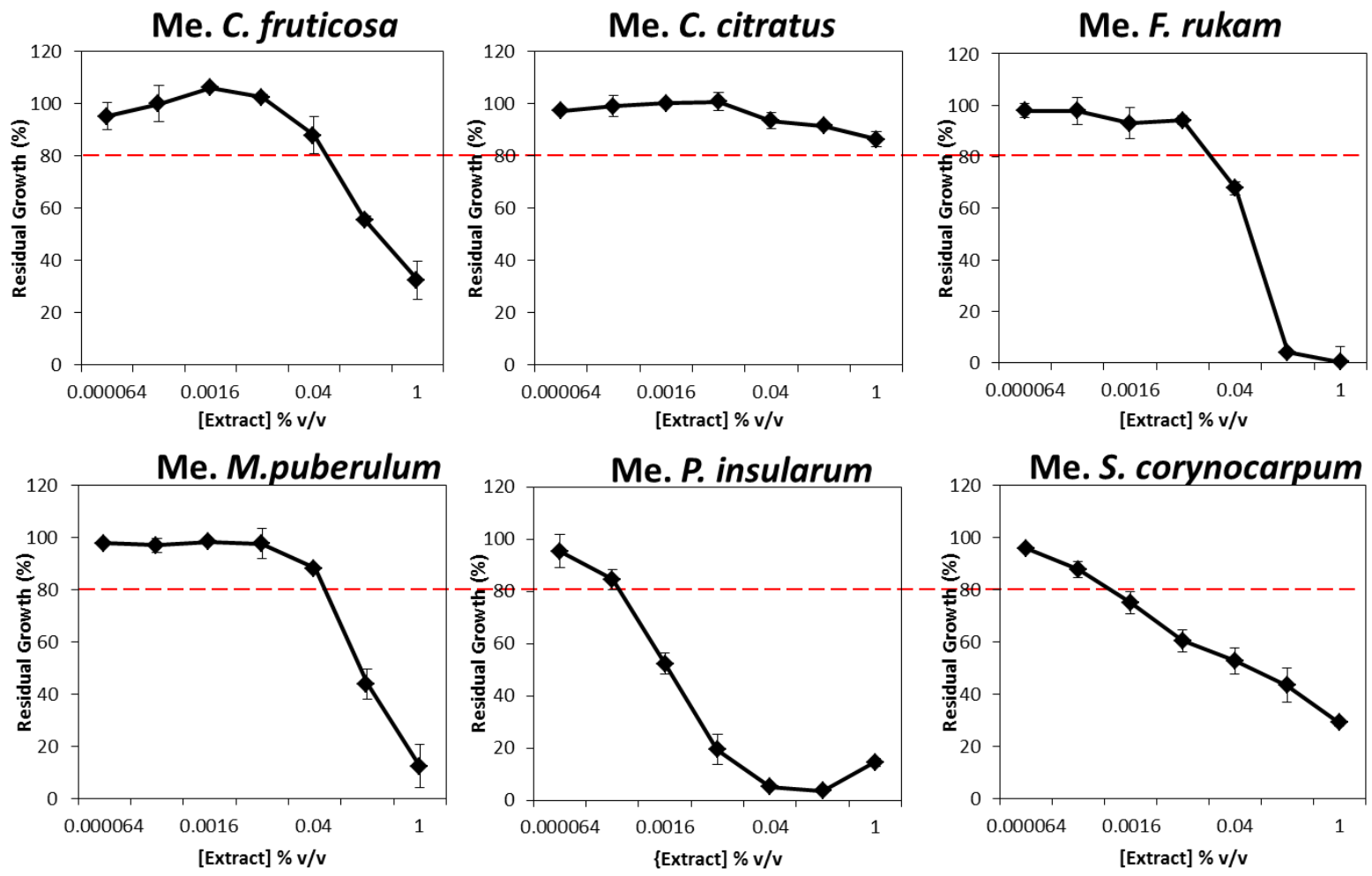

Me. P. insularum
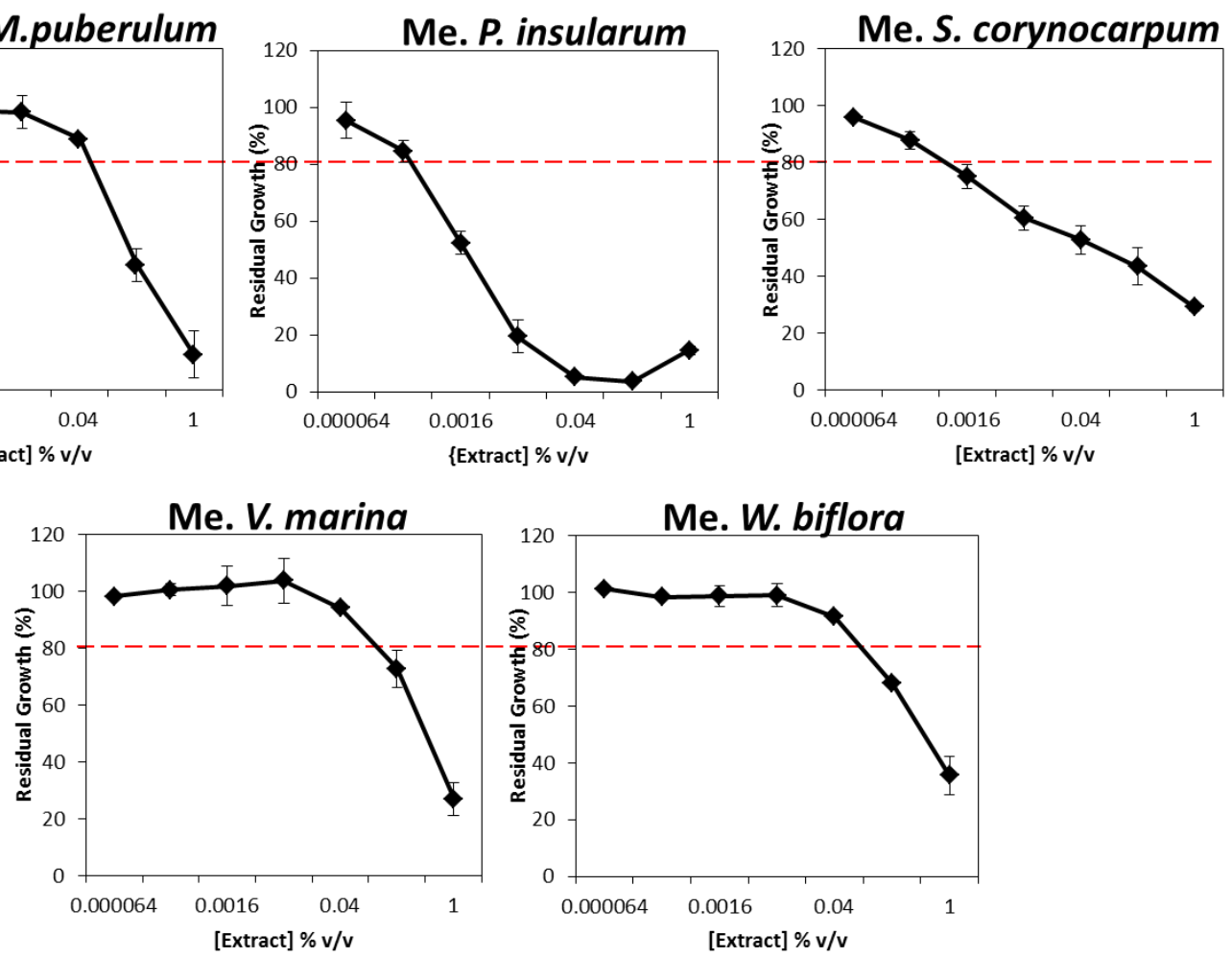

Figure 2.7: $\quad$ Liquid-based bioactivity assay of methanol extracts in pH 7.0 SC. Residual growth assays of the 11 methanol extracts of Samoan medicinal plants; cells inoculated into SC buffered to pH 7.0 with 25mM HEPES were treated with increasing concentrations of methanol extracts from $6.4 \times 10^{-5}$ to $1 \% \mathrm{v} / \mathrm{v}$, and growth was compared against DMSO control cells. Red dashed lines mark threshold for indication of bioactivity (i.e., residual growth decreasing lower than $80 \%$ and exhibiting greater than $20 \%$ growth inhibition). 


\subsubsection{Extracts from different plants exhibit varying bioactivity profiles}

To bring the extract bioactivity profile into context, the bioactivity results of the aqueous and methanolic extracts at two defined pH levels tested (Fig 2.4-2.7) were compiled (Table 2.1). The aqueous and methanolic extracts of $B$. javanica only demonstrated bioactivity in $\mathrm{SC}$ at pH 7.0 whilst only the methanolic extracts of $C$. fruticosa, $V$. marina and $W$. biflora were active in both $\mathrm{pH} 4.5$ and $\mathrm{pH}$ 7.0. The $C$. asiatica and $S$. corynocarpum methanol extracts were active at either pH levels, whilst the aqueous extracts did not exhibit any bioactivity. The extracts of $C$. inerme, F. rukam and $M$. puberulum were bioactive in three of the four conditions, although these conditions varied from plant to plant. For instance, the aqueous extract of $F$. rukam was active at $\mathrm{pH} 4.5$ and $\mathrm{pH} 7.0$, while its methanolic extract was only active at $\mathrm{pH}$ 7.0. Conversely, $C$. inerme and $M$. puberulum aqueous extracts were only active at $\mathrm{pH} 7.0$, and their methanolic extracts were active at both $\mathrm{pH} 4.5$ and $\mathrm{pH}$ 7.0. Only $P$. insularum extracts were bioactive across all four conditions, as the aqueous and methanolic extracts were bioactive at both $\mathrm{pH} 4.5$ and $\mathrm{pH}$ 7.0. In contrast, neither the aqueous nor the methanol extract from $C$. citratus reduced yeast growth at either $\mathrm{pH}$.

Table 2.1: Summary of bioactivity of aqueous and methanol extracts. The table summarizes the concentration $(\% \mathrm{v} / \mathrm{v})$ of extract required to inhibit $20 \%$ of yeast growth. NB indicates extracts which did not reduce yeast growth by $20 \%$ at the highest tested concentration. NT indicates extracts which were not tested. Red indicates lowest concentration producing bioactivity.

\begin{tabular}{r|ccc|ccc} 
& \multicolumn{3}{|c|}{ Aqueous Extract } & \multicolumn{3}{c}{ Methanolic Extract } \\
\cline { 2 - 7 } & Liquid pH 4.5 Liquid pH 7.0 & Agar pH 7.0 & Liquid pH 4.5 & Liquid pH 7.0 & Agar pH 7.0 \\
\hline B. javanica & 1.5 & 0.0016 & 4 & NB & 0.0032 & 0.2 \\
C. inerme & NB & 0.016 & 7 & 0.06 & 0.0064 & 0.2 \\
C. asiatica & NB & NB & NT & 0.35 & 0.05 & 0.1 \\
C. fruticosa & NB & NB & NT & NB & 0.08 & 0.2 \\
C. citratus & NB & NB & NT & NB & NB & NT \\
F. rukam & NB & NB & 5 & NB & 0.024 & 0.5 \\
M. puberulum & NB & 0.00032 & 15 & 0.6 & 0.08 & 0.2 \\
P. insularum & 0.024 & 0.000064 & 3 & 0.05 & 0.0005 & 0.5 \\
S. corynocarpum & NB & NB & NT & 0.2 & 0.00096 & 1 \\
V. marina & NB & NB & NT & NB & 0.12 & 0.2 \\
W. biflora & NB & 0.00032 & NT & NB & 0.08 & 1 \\
\hline & $5 / 11$ & $10 / 11$ & $5 / 11$ & $5 / 11$ & $10 / 11$ & $10 / 11$
\end{tabular}




\subsubsection{Aqueous extracts are not substrates of the PDR system}

Yeast undoubtedly is a powerful tool for investigating drug mechanism of action (Giaever et al., 2004; Parsons et al., 2004; Yibmantasiri et al., 2012). However, it is often the case that studies in yeast require higher concentrations of a drug than that required in mammalian cell studies (Coorey et al., 2015). Whilst the yeast cell wall contributes to this phenomenon, the major reason is the pleiotropic drug resistance (PDR) system (Coorey et al., 2015). The yeast PDR system, as the name suggests, functions to effectively remove toxic drug compounds out of the cell via $A B C$ transporters located in the plasma membrane. This defence system in yeast is regulated by two main transcription factors, $P D R 1$ and PDR3. Deletion of these transcription factors results in a strain with reduced drug reflux capabilities (Coorey et al., 2015).

To determine if the aqueous plant extracts were substrates of the PDR system, the $p d r 1 \Delta p d r 3 \Delta$ double deletion mutant was grown in the presence of increasing concentrations of plant extracts in $\mathrm{SC}$ at $\mathrm{pH}$ 7.0. The growth profile of $p d r 1 \Delta p d r 3 \Delta$ in the presence of the extracts was compared to the growth profile of WT (Fig 2.8). Differences were observed in the growth of WT and $p d r 1 \Delta p d r 3 \Delta$ in the different aqueous extracts. For instance, WT exhibited a lower growth profile (i.e., more sensitive growth profile) than $p d r 1 \Delta p d r 3 \Delta$ in the presence of the aqueous extract from $C$. asiatica. A similar difference between WT and the PDR attenuated strain was observed when treated with the aqueous extracts from C. fruticosa, C. citratrus, M. puberulum and V. marina. The differences between WT and $p d r 1 \Delta p d r 3 \Delta$ do not appear to be statistically significant. When treated with the aqueous extracts of B. javanica, C. inerme, F. rukam, P. insularum and W. biflora, the growth profiles of WT and pdr1 $\Delta p d r 3 \Delta$ were comparable. However, treatment with the aqueous extracts of $S$. corynocarpum resulted in a worse growth profile from $p d r 1 \Delta p d r 3 \Delta$ compared to $W T$, although the difference did not appear significantly different. 

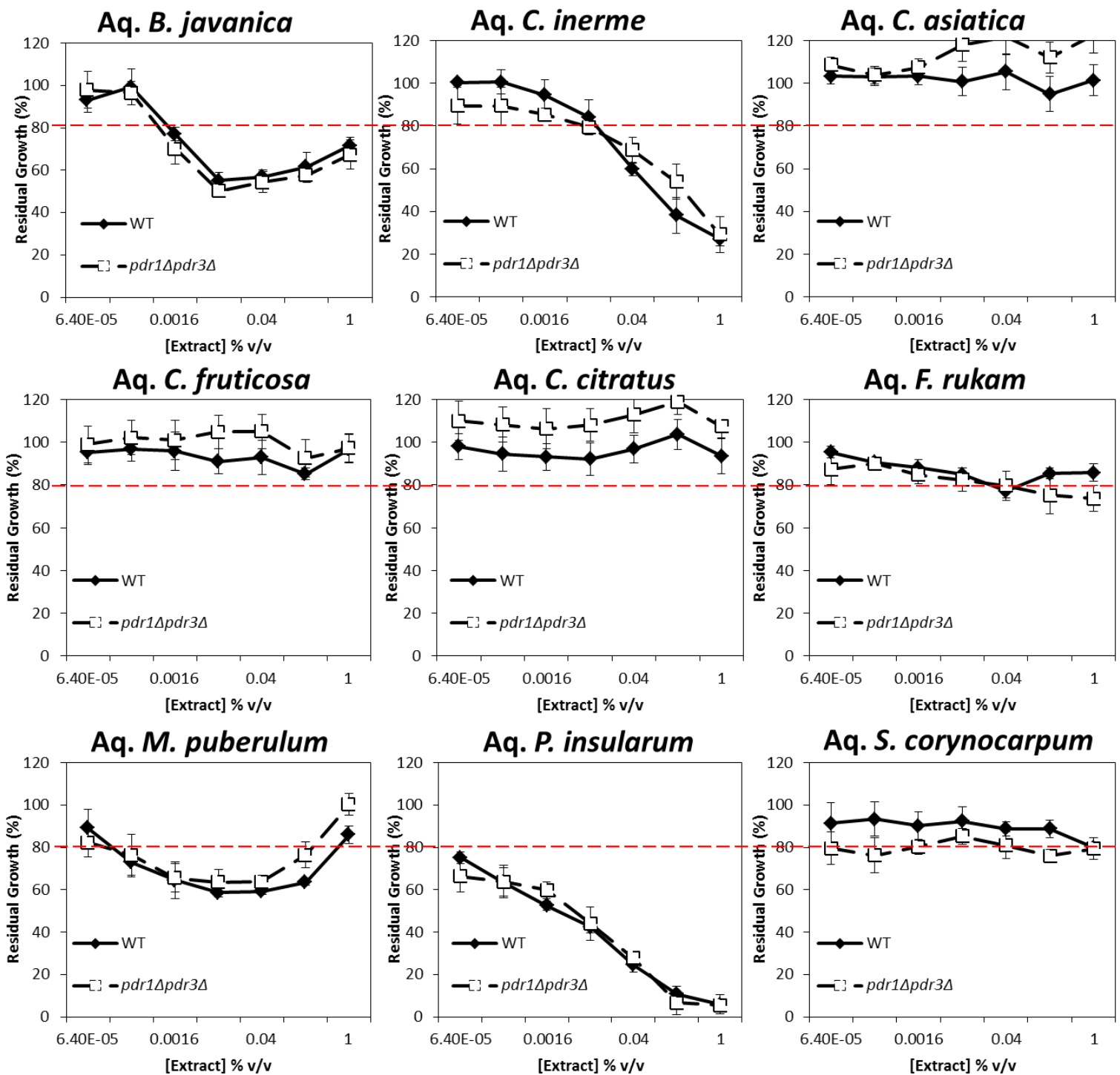

Aq. S. corynocarpum
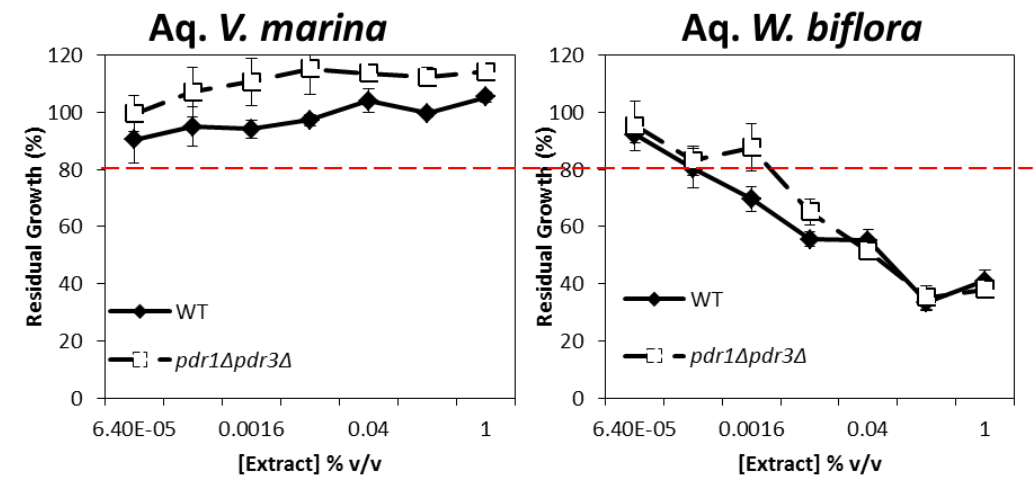

Figure 2.8: Liquid-based growth assays of Y7092 WT and PDR-attenuated strain in aqueous extracts. Residual growth assays of the 11 aqueous extracts of Samoan medicinal plants. Y7092 (WT) and $p d r 1 \Delta p d r 3 \Delta$ cells were inoculated into SC at pH 7.0 and treated with increasing concentrations of aqueous extracts from $6.4 \times 10^{-5}$ to $1 \% \mathrm{v} / \mathrm{v}$. Growth was compared against untreated cells, and residual growth calculated. Red dashed lines mark threshold for indication towards bioactivity (i.e., residual growth decreasing lower than $80 \%$ and exhibiting greater than $20 \%$ growth inhibition). 


\subsubsection{Methanolic extracts are not substrates of the PDR system}

To determine if the methanolic extracts obtained from the leaves of the selected Samoan medicinal plants were substrates of the yeast PDR system, Y7092 WT and $p d r 1 \Delta p d r 3 \Delta$ were grown in the presence of the methanolic extracts over a concentration range of $6.4 \times 10^{-5}$ to $1 \% \mathrm{v} / \mathrm{v}$ in SC at $\mathrm{pH}$ 7.0. SC at pH 7.0 media was maintained in this experiment because it enhanced bioactivity of the extracts compared to that obtained from SC at pH 4.5. The growth of both strains in the presence of the extract was compared to growth in the presence of the vehicle control ( $1 \% \mathrm{v} / \mathrm{v} \mathrm{DMSO})$ to determine their residual growth (Fig 2.9).

Different patterns of growth comparison between WT and pdr1 $\Delta p d r 3 \Delta$ were observed under treatment with methanolic extracts. For instance, treatment with the methanolic extracts from C. inerme, C. asiatica, C. fruticosa, C. citratus, F. rukam, S. corynocarpum, V. marina and W. biflora produced comparable growth profiles between WT and $p d r 1 \Delta p d r 3 \Delta$. However, there were three methanolic extracts that produced varying growth profiles between WT and $p d r 1 \Delta p d r 3 \Delta$, including the methanolic extracts from B. javanica, $M$. puberulum and $P$. insularum. However, while there appears to be statistical significance in some of these differences, whether there is biological relevance or not requires further investigation. Across all the extracts though, none produced a pdr1 1 pdr3 $\Delta$ growth profile that was more sensitive than $\mathrm{WT}$, suggesting that the methanolic extracts were not substrates of the PDR system. 


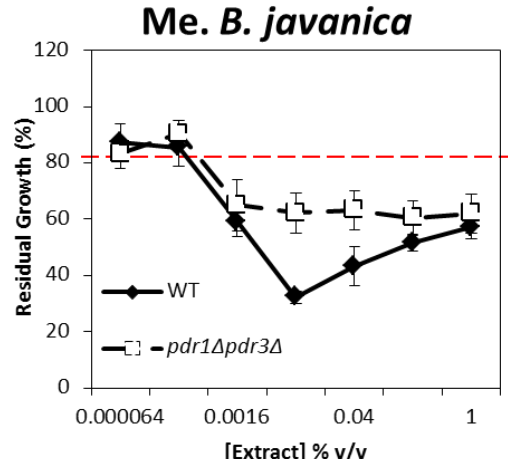

Me. C. fruticosa

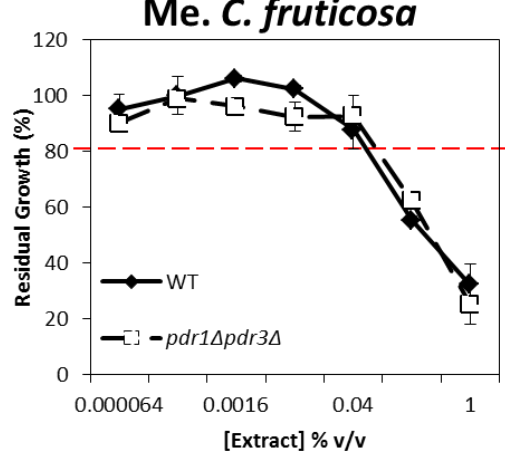

Me. M. puberulum

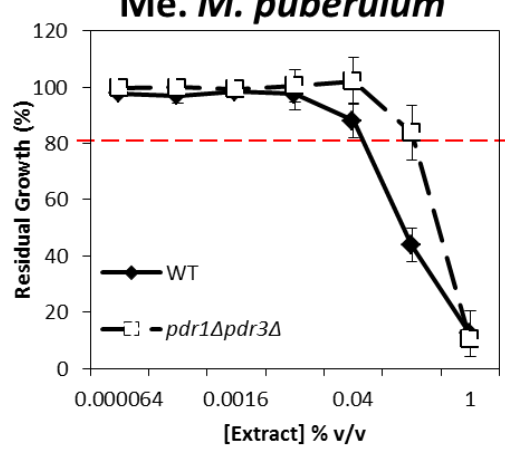

Me. C. inerme

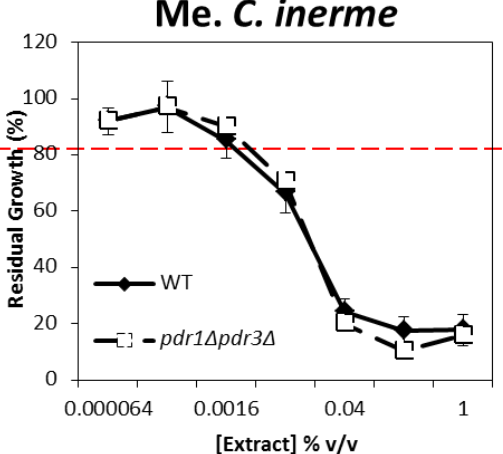

Me. C. citratus

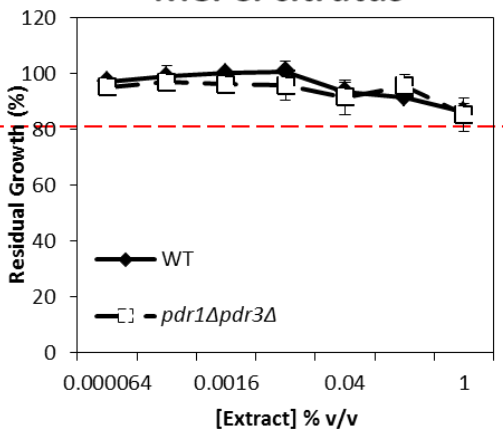

Me. P. insularum

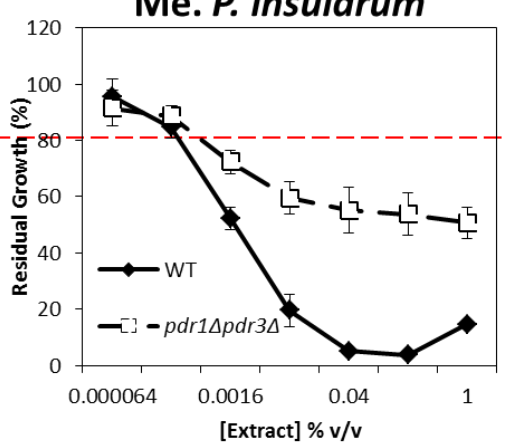

Me. C. asiatica

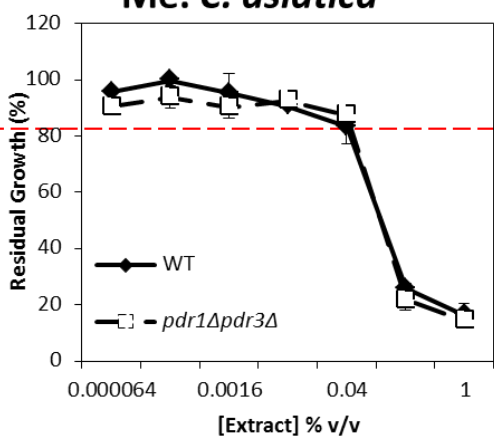

Me. F. rukam

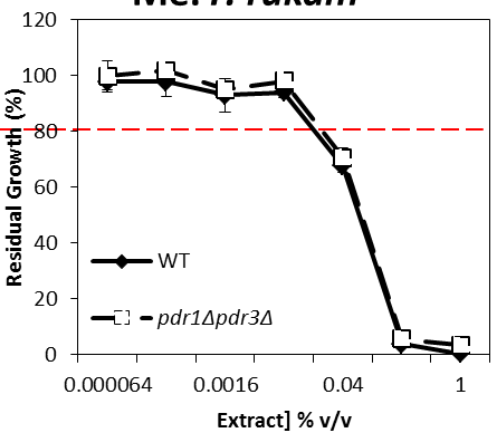

Me. S. corynocarpum

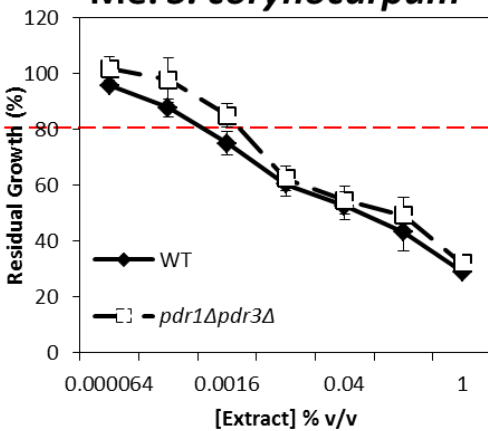

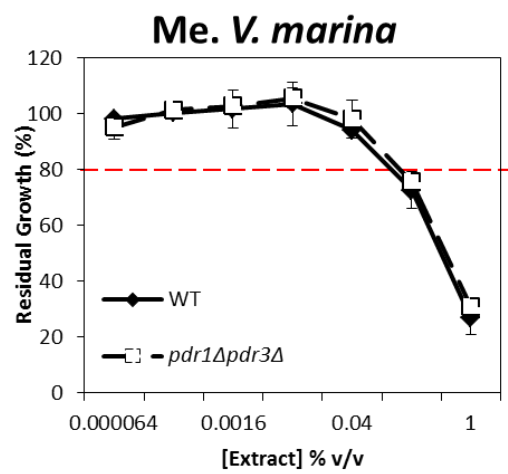

Me. W. biflora

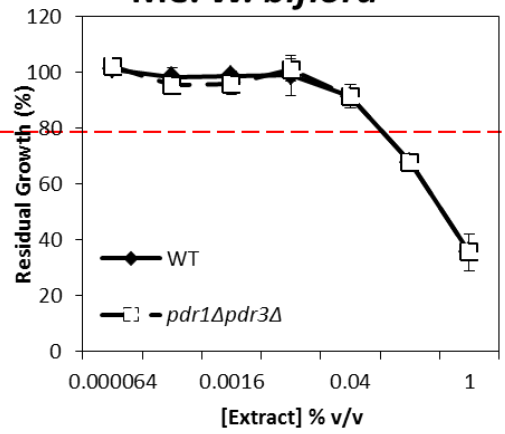

Figure 2.9: Liquid-based growth assays comparing growth profiles in WT and PDR-attenuated strain in methanol extracts. Residual growth assays of the 11 methanol extracts of Samoan medicinal plants. Y7092 (WT) and pdr1 $\Delta p d r 3 \Delta$ cells were inoculated into SC buffered to pH 7.0 with $25 \mathrm{mM}$ HEPES, and treated with increasing concentrations of methanol extracts from $6.4 \times 10^{-5}$ to $1 \% \mathrm{v} / \mathrm{v}$. Growth was compared against vehicle control cells, and residual growth was calculated. Red dashed lines mark threshold for indication of bioactivity (i.e. residual growth decreasing lower than $80 \%$ and exhibiting greater than $20 \%$ growth inhibition). 


\subsubsection{Aqueous extracts maintain bioactivity in agar}

Performing genome-wide analyses using the yeast deletion library on agar is more cost effective than performing microarray- or barcode sequencing-based genomic analyses. However, extracts that show bioactivity in liquid may not necessarily display bioactivity in agar, nor may there be sufficient compound depending on the potency of the compound in agar. To determine whether the extracts were amenable to agar-based genome-wide analyses, we ascertained if extract bioactivity was maintained in agar-based growth assays. This was achieved by spotting BY4741 cells on agar containing various concentrations of one of the five bioactive aqueous extracts, and comparing their growth to BY4741 grown in the vehicle control (Fig 2.10).

Although the extracts exhibited bioactivity at low concentrations in liquid, these concentrations did not reduce yeast growth in agar (data not shown). The experiment was therefore carried out at higher concentrations of the extracts to induce a growth defect. It was observed that B. javanica reduced growth of BY4741 at the $4 \% \mathrm{v} / \mathrm{v}$ concentration. As concentrations of aqueous $B$. javanica extract increased, BY4741 growth improved, similar to the profile observed in the liquidbased assays. In the cases of $C$. inerme, F. rukam, M. puberulum and P. insularum, BY4741 growth decreased in a dose-dependent manner. The aqueous extract from $C$. inerme reduced growth at $7 \%$ $\mathrm{v} / \mathrm{v}$. The F. rukam aqueous extract inhibited growth at $5 \% \mathrm{v} / \mathrm{v}$, whilst this was only achieved at $15 \%$ $\mathrm{v} / \mathrm{v}$ of the aqueous $M$. puberulum extract. In contrast, the $P$. insularum aqueous extract inhibited BY4741 growth at $3 \% \mathrm{v} / \mathrm{v}$. These results show that $P$. insularum required the least amount of extract to inhibit growth in an agar-based growth assay $(3 \% \mathrm{v} / \mathrm{v})$, followed by the $B$. javanica aqueous extract at $4 \% \mathrm{v} / \mathrm{v}$. The $F$. rukam extract is ranked third in bioactivity with a requirement of $5 \%$ of its aqueous extract to inhibit growth, and the $C$. inerme aqueous extract is ranked fourth via growth inhibition at $7 \% \mathrm{v} / \mathrm{v}$. M. puberulum was the least bioactive requiring $15 \% \mathrm{v} / \mathrm{v}$ to inhibit yeast growth. 


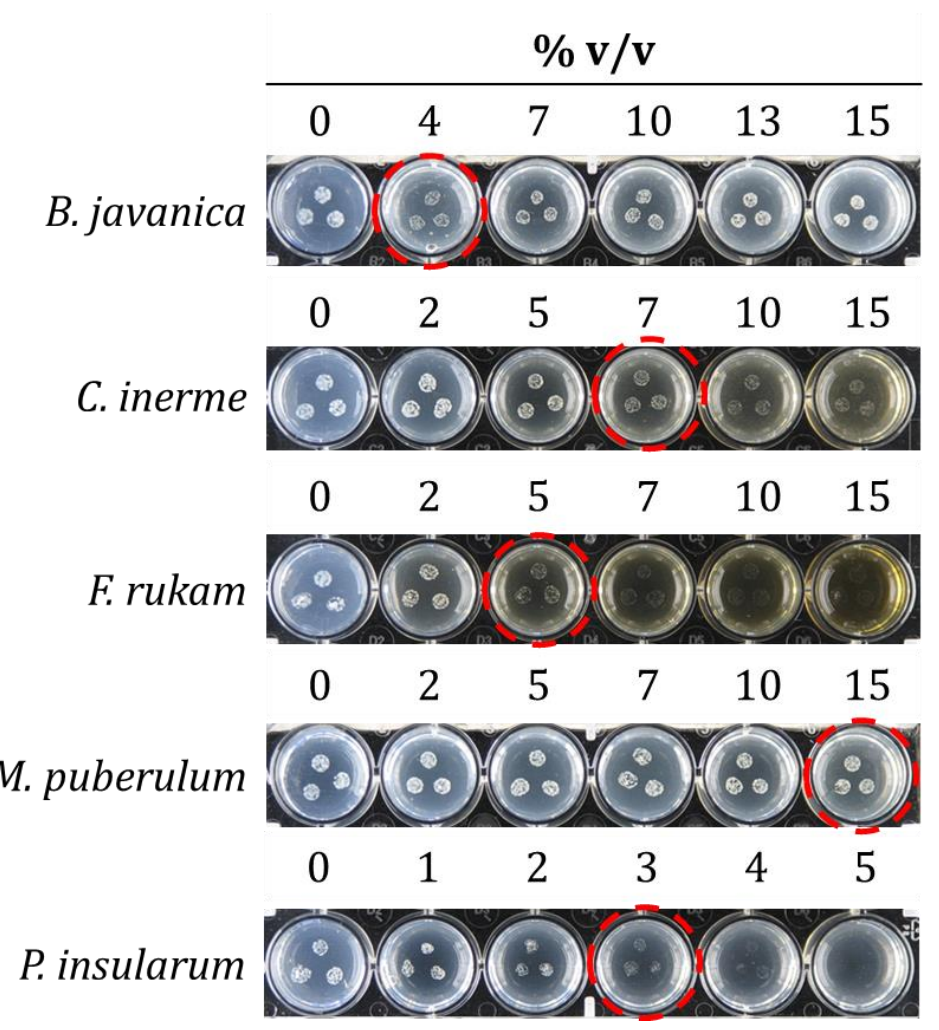

Figure 2.10: Agar-based growth assays of WT in aqueous extracts. WT yeast strain was grown on agar in the presence or absence of aqueous plant extracts. Agar was inoculated with yeast in spots. Growth was then compared between control agar well $(0 \% \mathrm{v} / \mathrm{v})$ and wells with various concentrations of extracts. Wells where aqueous extracts start to induce a growth defect are highlighted with red-dashed circles. Different volumes of extract had the relevant volume of vehicle control, but did not induce any change (data not shown) and a representative well is shown as control $(0 \% \mathrm{v} / \mathrm{v}$ extract).

\subsubsection{Methanolic extracts maintain bioactivity in agar}

To determine if the bioactivity of methanol extracts were stable in agar and thus amenable to agar-based genome-wide analyses, we performed an experiment similar to that described above for aqueous extracts, utilizing increasing concentrations of the 10 bioactive methanolic extracts (Fig 2.11). The same phenomenon observed in the aqueous extracts whereby higher concentrations of extract was required to induce a growth defect in agar was again observed (data not shown), and the concentrations tested were therefore increased for these experiments. The methanolic extracts of B. javanica, C. inerme, C. fruticosa, M. puberulum and V. marina reduced growth of BY4741 at $0.2 \% \mathrm{v} / \mathrm{v}$ on agar. The methanolic extract of $C$. asiatica produced minimal growth reduction of 
BY4741 at $0.04 \% \mathrm{v} / \mathrm{v}$ and completely inhibited its growth at $0.2 \% \mathrm{v} / \mathrm{v}$, thus a titration experiment across the range of $0.04 \% \mathrm{v} / \mathrm{v}$ and $0.2 \% \mathrm{v} / \mathrm{v}$ to determine the concentration to detect approximately 10-20\% growth inhibition of BY4741 (the amount of inhibition required for genome-wide analysis on agar) should work proceed with this extract. The same case was observed for both $F$. rukam and $P$. insularum, requiring concentration titration across the range of $0.2 \% \mathrm{v} / \mathrm{v}$ and $1 \% \mathrm{v} / \mathrm{v}$. Methanolic extractions from $S$. corynocarpum and $W$. biflora were required at $1 \% \mathrm{v} / \mathrm{v}$ to induce growth inhibition of BY4741 on agar. Interestingly, while the methanolic extract of $P$. insularum was the most potent in liquid-based growth assays, it did not appear to be the most potent in agar, suggesting reduced activity of the extract in this form of growth assay.

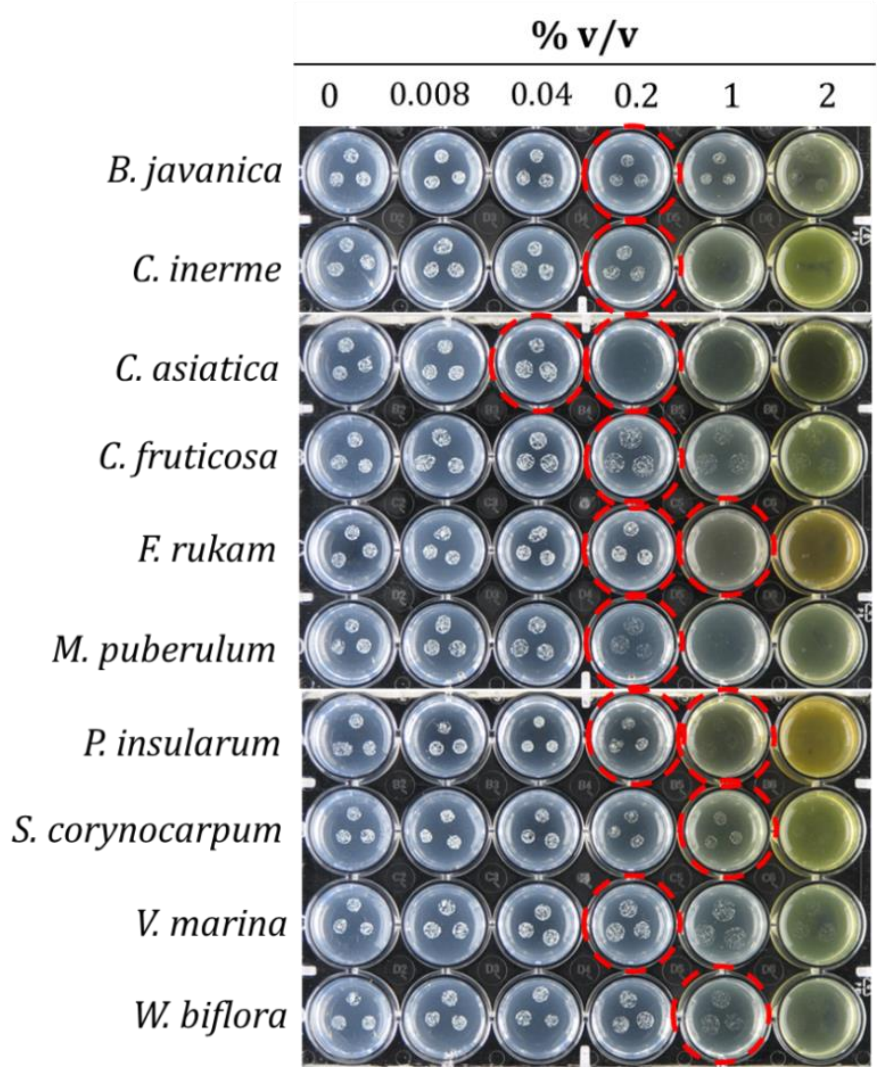

Figure 2.11: Agar-based growth assays of WT against methanol extracts. WT yeast strain was grown on agar in the presence or absence of plant methanol extracts. Agar was inoculated with yeast in spot-format. Growth was then compared between BY4741 WT on control agar well ( $0 \% \mathrm{v} / \mathrm{v})$ and BY4741 WT in wells with various concentrations of extracts. Wells with extracts exhibiting a visually detectable reduction in growth are highlighted with red-dashed circles. Two circles per extract indicate a requirement to titrate between two concentrations. 


\subsection{Discussion}

Our findings have clearly demonstrated that most of the 11 Samoan medicinal plants displayed bioactivity in yeast. Of the 22 extracts prepared from the 11 plants, five aqueous and 10 methanolic extracts inhibited yeast growth, a $68 \%$ bioactivity rate (Table 2.1). Previous pharmacological screens of Samoan medicinal plants for bioactivity produced varied bioactivity rates; Norton et al. (1973) had a 58\% bioactivity rate from 208 plant extracts, Cox et al. (1989) had a 78\% bioactivity rate from 104 plant extracts, and Dunstan et al. (1997) tested 50 Samoan medicinal plant extracts and had a $32 \%$ bioactivity rate, making the bioactivity rate obtained here comparable to those previous studies. However, studies previously carried out on Samoan medicinal plants utilized multiple assays to determine bioactivity (e.g., Dunstan et al. (1997) used an in vitro assay assessing prostaglandin biosynthesis and rat ear oedema) which may subsequently result in a higher bioactivity rate, compared to the study reported herein, whereby only one assay (yeast growth inhibition) was used to evaluate bioactivity. In this context, our study on Samoan medicinal plants produced a higher bioactivity rate than those previously reported. A study on 61 Indian plant extracts produced a $34 \%$ bioactivity rate (Kumar et al., 2006), suggesting that the bioactivity rate detected from the yeast liquid-based assay carried out in this work on Samoan plant extracts was comparable to studies of plants outside of Samoa. It is important to note that the bioactivity detected here from the crude aqueous and methanolic extracts of the selected medicinal plants, may be a consequence of a single bioactive compound, a mixture of compounds, or the synergistic activities of more than one compound. Also of significance from these results was the conclusion that only $C$. citratus produced aqueous and methanolic extracts that did not reduce yeast growth in $\mathrm{SC}$ at $\mathrm{pH} 4.5$ and $\mathrm{pH}$ 7.0. However, this conclusion was drawn on the basis of the parameters tested here, and that $C$. citratus extracts may be bioactive if tested at higher concentrations, in a different system, or if extracted using a different solvent. 
Also of note is the significantly higher concentration of extracts necessary to inhibit yeast growth in agar compared to that in liquid. For instance, $6.5 \times 10^{-5} \% \mathrm{v} / \mathrm{v}$ of $P$. insularum aqueous extract was required to inhibit approximately $20 \%$ of BY4741 growth in liquid (Fig 2.5) whilst in the agar assay, it was required at $3 \% \mathrm{v} / \mathrm{v}$ to induce a similar level of growth inhibition (Fig 2.10). One explanation could be the $50^{\circ} \mathrm{C}$ temperature needed to liquefy agar, however extract activity was maintained after heating at $70^{\circ} \mathrm{C}$ overnight for $15 \mathrm{~h}$ (Appendix II). A more likely explanation is the form of the assay because in liquid, yeast cells are surrounded by media containing the tested extracts whereas in agar, the plant extracts are in much more limited contact with yeast cells.

Bioactivity of the plant extracts was affected when HEPES was added to buffer pH from 4.5 to 7.0, regardless of whether the extracts were aqueous or methanolic. For instance, the aquoues extracts of $\mathrm{C}$. inerme that did not inhibit yeast growth at $\mathrm{pH} 4.5$ reduced yeast growth at $\mathrm{pH}$ 7.0. Furthermore, methanolic extracts that were shown to have marginal inhibitory effects on yeast growth at $\mathrm{pH} 4.5$ showed increased inhibition at $\mathrm{pH} 7.0$, such as the methanolic extracts from $B$. javanica and C. fruticosa. Whether this phenotype is common with all plant extracts remains unknown, as most studies into plant crude extracts have not considered $\mathrm{pH}$ as a variable (AlanisGarza et al., 2007; Ali-Shtayeh \& Ghdeib, 1999; Hammer et al., 1999; Kumar et al., 2006; Quiroga et al., 2001; Rauha et al., 2000; Satish et al., 2007; Webster et al. 2008; Weckesser et al., 2007; Wilson et al., 1997). This aside, these results suggest that either $\mathrm{pH} 7.0$ or the HEPES buffer used increased potency of the extracts. Importantly, Quek et al. (2013) showed that pH does affect potency, when pH 7.0 reduced the bioactivity of their compound TA-289 2-fold compared to its activity at pH 4 . Whilst this trend is opposite to the one observed here, it shows that $\mathrm{pH}$ does affect potency of bioactive compounds. Further, Tanaka et al. (1996) showed the activity of the antimicrobial agent DU-6859a was also affected by $\mathrm{pH}$, where its MIC was reduced as $\mathrm{pH}$ increased. Furthermore, Palm et al. (1999) showed that $\mathrm{pH}$ affected drug transport. These works provide evidence that $\mathrm{pH}$ does affect the potency however, to fully discount the possibility that the increased potency was a 
consequence of HEPES addition, we propose the $\mathrm{pH}$ of the media be adjusted to 7.0 using a different buffer (e.g., MOPs) or using $\mathrm{NaOH}$.

Another promising finding from the results presented in this chapter is the lack of hypersensitivity of the PDR-attenuated strain $(p d r 1 \Delta p d r 3 \Delta)$ to the extracts of the Samoan medicinal plants. Although hypersensitivity would have allowed further studies to be carried out at lower concentrations (Coorey et al., 2015), it would have also indicated extract unsuitability for application as antifungals. However, our findings suggested that the bioactive compounds in the plant extracts were not substrates of the yeast drug efflux system, further inferring that the plant extracts could be exploited as antifungals. Intriguingly, whilst the PDR attenuated strain did not appear hypersensitive to the extracts, in some cases, they appeared more resistant towards the extracts, as the WT exhibited more sensitivity. This suggests that PDR1 and/or PDR3 contribute to the mechanism of action of the extracts, such as the methanolic extracts of $B$. javanica, M. puberulum and $P$. insularum.

In summary, 15 out of 22 extracts from 11 Samoan medicinal plants were established as bioactive in S. cerevisiae, and the optimal media condition for the most potency was elucidated. The amenability of the extracts for agar-based genome-wide analyses was also determined, and the extracts were further shown to not be substrates of the PDR system. From this chapter, it was also ascertained that $P$. insularum was the only medicinal plant that produced bioactivity across the aqueous and methanolic extracts at two different pH levels. The $P$. insularum extracts were the most potent aqueous extract and thus became the medicinal plant of most interest for the remainder of this thesis. 


\section{Chapter 3: \\ The Mechanism of Action of Psychotria insularum is Iron Chelation}

\subsection{Introduction}

Plants have been the source of many significant contributions to human medicine, including drugs such as aspirin and paclitaxel. Plants with extensive ethno-botanical background are even more successful as sources of drugs, for instance digoxin, quinine, morphine and artemisinin. However, a large portion of medicinal plants remain unstudied, and the mechanisms of action (MOA) of the majority remaining are largely unknown. Samoan medicinal plants were the subject of several investigative endeavours in the 1970s and 1980s (Cox et al., 1989; Norton et al., 1973). However, only their activities were explored, but how the plant extracts mediated their activity, or more specifically their MOA, remains largely unknown.

In Chapter 2, leaf extracts of the Samoan medicinal plant Psychotria insularum produced the most potent inhibition of yeast growth. Leaf extracts of $P$. insularum are one of the most prescribed medicinal remedies in Samoan traditional medicine against supernaturally induced ailments (Whistler, 1996). The plant is also administered in cases involving inflammation, such as fever, abdominal distress, skin infections, wounds, as well as general body aches and swellings (Dunstan et al., 1995; Whistler, 1996). Extracts of $P$. insularum reduced blood pressure, motor activity, prostaglandin synthesis and the development of rat ear oedema (Cox et al., 1989; Dunstan et al., 1995; Norton et al., 1973). Despite these multiple studies, the underlying MOA, notably the genes and pathways of how the extracts mediate these activities, are unknown.

The findings in the preceding chapter demonstrated that $P$. insularum was the only plant that produced an aqueous extract that was bioactive at both $\mathrm{pH} 4.5$ and $\mathrm{pH}$ 7.0. Further, the aqueous extract of $P$. insularum was the most potent in agar compared to other aqueous extracts. As 
aqueous extracts are of particular interest in this thesis in the interest of correlating MOA to application in traditional Samoan medicine, the genetic analysis utilizing the Mata haploid deletion library of S. cerevisiae was carried out on the aqueous extract of $P$. insularum. We elucidated that $P$. insularum extracts possessed iron-chelating activity regulated an anti-inflammatory activity, correlating its MOA to how the plant is used in the Samoan traditional medicine setting. 


\subsection{Methods}

\subsubsection{Agar-based genome-wide analysis}

Rectangular petri plates (Singer Instrument Co. Ltd) were prepared with $40 \mathrm{~mL}$ of SC agar containing either the relevant concentration of the aqueous extract of $P$. insularum or water. The Mata haploid deletion mutants subset of the Yeast Knockout Collection (Open Biosystems) in the 1,536 format (i.e. 1,536 colonies per plate arrayed as quadruplicates of 384 mutants) was pinned in triplicate on control agar and on agar containing the aqueous extract. The plates were incubated at $30^{\circ} \mathrm{C}$ for $24 \mathrm{~h}$, photographed using a Canon Powershot S3IS digital camera, further incubated for an additional $24 \mathrm{~h}$, and photographed again. For each time point (24 and $48 \mathrm{~h}$ ), colony sizes were measured using Gitter (Wagih \& Parts, 2014) and ScreenMill (Dittmar et al., 2010) was used to quantify colony fitness of each gene deletion strain including statistical evaluation using z-scores and p-values. To confirm mutant growth was reduced in the presence of the extract, all deletion mutant strains with $p$-values less than 0.05 in two or more replicates were selected for validations via serial dilution growth analyses. Five-fold serial dilutions of $5 \times 10^{5}$ cells $/ \mathrm{mL}$ were spotted on SC agar containing aqueous extract of $P$. insularum or vehicle control. Plates were incubated at $30^{\circ} \mathrm{C}$ for $24 \mathrm{~h}$, photographed, further incubated for an additional $24 \mathrm{~h}$, and photographed again.

\subsubsection{Metal rescue assay}

Amelioration of $P$. insularum-induced growth defects was evaluated with the addition of various metals. Cultures were prepared by inoculating experimental cultures at $5 \times 10^{5}$ cells $/ \mathrm{mL}$ in SC with the presence or absence of $100 \mu \mathrm{M}$ of metal solutions of interest $\left(\mathrm{FeCl}_{3}, \mathrm{FeSO}_{4}, \mathrm{ZnCl}_{2}, \mathrm{CaCl}_{2}\right.$, $\mathrm{CuCl}_{2}, \mathrm{MgCl}_{2}$ and $\mathrm{MnCl}_{2}$ ). Concurrently, cultures with and without exogenous metal supplementation were treated with increasing concentrations of $P$. insularum extract, incubated at $30^{\circ} \mathrm{C}$ for $15 \mathrm{~h}$, and quantified via absorbance at $590 \mathrm{~nm}$ using an EnVision 2102 multilabel plate reader (Perkin Elmer). 
Percentage of residual growth (treated $\mathrm{OD}_{590}$ /untreated $\mathrm{OD}_{590} \times 100$ ) was then calculated for each treatment.

\subsubsection{Quantification of intracellular iron}

Intracellular iron was quantified using inductively coupled plasma mass spectroscopy (ICPMS) as previously described (Tamarit et al., 2006). Cultures were prepared by inoculating experimental cultures at $5 \times 10^{5} \mathrm{cells} / \mathrm{mL}$ in SC with the relevant treatment (extracts or controls at the prescribed concentration). At mid-log growth, $10 \mathrm{OD}$ units were collected by centrifugation. Cells were digested in $3 \%$ nitric acid at $96^{\circ} \mathrm{C}$ for $16 \mathrm{~h}$ and clarified by centrifugation at $13,000 \mathrm{rpm}$ for 5 min. Levels of iron, copper, zinc, calcium and magnesium were measured in the supernatant using ICP-MS (Element 2 Magnetic Sector Field, ThermoFisher). Metal standards were run in conjunction with samples, as well as the relevant equipment blanks to monitor potential contamination at each step of the process. Spike samples were also prepared to ensure that cell lysate was not interfering with metal quantification, and each sample was analysed by ICP-MS 10 times. Where changes were observed, the statistical significance of an increase or decrease was assessed using a one-tailed Student's t-test, and the same was carried throughout this thesis.

\subsubsection{Protein expression}

Protein expression was quantified by fluorescence of a GFP-tagged protein of interest relative to the fluorescence of internal markers of the cytoplasm and nucleus (mCherry and RedStar, respectively). Experimental cultures were prepared by inoculating with $5 \times 10^{5}$ cells $/ \mathrm{mL}$ of the relevant strain (Appendix III) and grown in the relevant treatment and grown at $30^{\circ} \mathrm{C}$ for $15 \mathrm{~h}$, diluted to approximately $1 \times 10^{9}$ cells $/ \mathrm{mL}$, transferred to 384 clear bottom microtitre plates (Perkin Elmer), and visualized using the Evo-Tec OPERA high throughput spinning disc confocal microscope (Perkin Elmer) at room temperature, with the 60X water immersion lens (NA 1.2). GFP excitation was at 488 $\mathrm{nm}$ and detected with a 520/35 filter. mCherry and RedStar excitation was at $561 \mathrm{~nm}$ and detected 
with a $600 / 40$ filter. An exposure of $400 \mathrm{~ms}$ with 5 Z-stacks $0.5 \mu \mathrm{m}$ apart was used for all images (Bircham et al., 2011). GFP quantification was achieved using ACAPELLA software v2.0 (Perkin Elmer) that identified cells based on the mCherry and RedStar signlas and the quantification of GFP as previously described (Bircham et al., 2011). Fluorescence was compared between treatments using a Student's t-test.

\subsubsection{Heme assay}

Heme was quantified by the triton-methanol protocol as previously described (Pandey et al., 1999). Cells were treated overnight as described before, lysed by glass-bead agitation, and protein extracted as described in Dunn \& Wobble (2001) and Zhang et al. (2011). Briefly, cells were washed, pre-treated with $2 \mathrm{M}$ lithium acetate, washed, pre-treated with $0.4 \mathrm{M} \mathrm{NaOH}$, before lysis with acid-washed glass beads. The cell lysate was then collected by centrifugation. Protein was quantified using the BCA protein assay kit (Pierce) according to manufacturer instructions. Heme was then quantified by the triton-methanol protocol (Pandey et al., 1999) where $20 \mu \mathrm{L}$ of clarified cell lysate was added to $180 \mu \mathrm{L}$ triton-methanol (2.5\% triton in methanol), and absorbance was read at $405 \mathrm{~nm}$ using an Envision plate reader (Perkin Elmer). Hemin (Sigma) was prepared at concentrations ranging from 10-500 $\mathrm{nM}$ for the standard curve. Heme content of yeast protein samples was calculated using the hemin standard curve, normalized to protein content, and reported as relative heme content [(treated heme $\mathrm{nM}_{\mathrm{n} / \mathrm{ug} \text { protein }} /$ untreated heme $\left._{\mathrm{nM} / \mathrm{ug} \text { protein }}\right) \times 100$ ].

\subsubsection{Aconitase assay}

Aconitase activity was measured using the Aconitase Assay Kit (Cayman Chemical) according to manufacturer's instructions. Briefly, RAW 264.7 cells were washed in cold 1 X PBS, resuspended in Assay Buffer and lysed by sonication 20 times with $5 \mathrm{~s}$ bursts. The cell suspension was then centrifuged at $500 \mathrm{xg}$ for $10 \mathrm{~min}$ at $4^{\circ} \mathrm{C}$ to remove cell debris and the supernatant was transferred to a fresh tube and frozen at $-80^{\circ} \mathrm{C}$ until use. Then $50 \mu \mathrm{L}$ of sample containing $1 \mathrm{mg} / \mathrm{mL}$ 
protein was aliquoted in a 96-well plate in triplicate, followed by the addition of $5 \mu \mathrm{L}$ Assay Buffer, $50 \mu \mathrm{L}$ of $\mathrm{NADP}^{+}$Reagent, $50 \mu \mathrm{L}$ isocitric dehydrogenase and $50 \mu \mathrm{L}$ substrate solution. A positive control was prepared using a manufacturer-supplied aconitase control, and blank wells were also prepared. Absorbance was measured at $340 \mathrm{~nm}$ every $30 \mathrm{~min}$ at $37^{\circ} \mathrm{C}$. Aconitase activity was then calculated using the following equation:

Aconitase Activity (nmol/min/mL) $=\left[\frac{\Delta \mathrm{A} / \mathrm{min}(\text { sample) }-\Delta \mathrm{A} / \mathrm{min} \text { (sample background) }}{0.00313 \mu \mathrm{M}^{-1}}\right] \times \frac{0.205 \mathrm{~mL}}{0.05 \mathrm{~mL}} \times$ Sample Dilution wherein $\Delta \mathrm{A}$ is the change in absorbance, $0.00313 \mu \mathrm{M}^{-1}$ is the extinction coefficient of aconitase based on pathlength of the well, $0.205 \mathrm{~mL}$ is the final reaction volume, and $0.05 \mathrm{~mL}$ is the aconitase sample used. Aconitase activities were ultimately compared between different treatments and the control.

\subsubsection{Iron chelation assay}

Iron chelation was directly measured using a modified chrome azurol S (CAS) assay as previously described (Alexander \& Zuberer, 1991). Briefly, the CAS assay solution was prepared by the addition of $7.5 \mathrm{~mL}$ of $2 \mathrm{mM} \mathrm{CAS}$ and $1.5 \mathrm{~mL}$ of $1 \mathrm{mM} \mathrm{FeCl}_{3}$ dissolved in $10 \mathrm{mM} \mathrm{HCl}$ to $21.9 \mathrm{mg}$ of HDTMA (hexa-decyl-tri-methy-ammonium bromide) dissolved in $25 \mathrm{~mL}$ water. To this solution, MES (2-( $\mathrm{N}$-morpholino)ethanesulfonic acid) buffer ( $\mathrm{pH}$ 5.6) was added and made up to $100 \mathrm{~mL}$ with $\mathrm{dH}_{2} \mathrm{O}$. Then $100 \mu \mathrm{L}$ of the prepared CAS solution was aliquoted into a 96-well plate, to which $100 \mu \mathrm{L}$ of various dilutions of the extracts were added. A colour change reaction from blue to yellow was indicative of iron chelation activity.

\subsubsection{Anti-inflammatory assays}

Markers of inflammation were measured using enzyme-linked immunosorbent assays (O'Sullivan et al., 2014). Briefly, bone marrow-derived macrophages from C57BL/6 mice (for MTT, cytokine and NO work) and RAW 264.7 cells (for aconitase work) were seeded at $1 \times 10^{5}$ or $5 \times 10^{4}$ 
cells/well respectively in 96-well plates, primed with $20 \mathrm{U} / \mathrm{mL}$ interferon gamma (IFN $\gamma$ ) and incubated at $37^{\circ} \mathrm{C}$ with $5 \% \mathrm{CO}_{2}$ for $18 \mathrm{~h}$, before $200 \mathrm{ng} / \mathrm{mL}$ LPS and relevant treatment were added simultaneously to each well. Plates were incubated for a further $24 \mathrm{~h}$, before $170 \mu \mathrm{L}$ of supernatants were collected for cytokine and NO analyses, and cells were used for MTT (3-[4,5-dimethylthiazoyl2-yl]-2,5-diphenyltetrazolium bromide) assays.

The MTT assay measures the metabolic activity of the cells, using the MTT tetrazolium salt which is converted from yellow to purple via the activity of metabolically active cells. Essentially, upon removal of supernatant for cytokine and NO analyses, $50 \mu \mathrm{L}$ of CTCM (complete T cell media) and $20 \mu \mathrm{L}$ of $5 \mathrm{mg} / \mathrm{mL} \mathrm{MTT}$ solution in PBS were added to the 96 -well plate. The plates were incubated at $37^{\circ} \mathrm{C}$ with $5 \% \mathrm{CO}_{2}$ for $2 \mathrm{~h}$, before $100 \mu \mathrm{L}$ of MTT solubilizer solution (10\% SDS [sodium dodecyl sulfate], $45 \%$ DMF [dimethylformamide] in $\mathrm{ddH}_{2} \mathrm{O} \mathrm{pH}$ 4.5) was added. Plates were incubated overnight at $37^{\circ} \mathrm{C}$, before reading at 570nm using an Enspire Multi-Plate reader (Perkin Elmer). NO is an indicator of the inflammatory response, and is rapidly degraded to nitrite and nitrate in the supernatant. These products are measured as an indicator of NO production in macrophages. This was achieved by mixing $50 \mu \mathrm{L}$ of cell supernatant with $50 \mu \mathrm{L}$ of Griess reagents in 96-well plates, together with a standard curve prepared from $\mathrm{NaNO}_{2}$ (sodium nitrite). Absorbance was read at 570nm. Enzyme-linked immunosorbent assays (ELISA) were used to determine levels of IL-10, IL-12 and TNF $\alpha$ from supernatants, conducted as per manufacturer's instructions (BD Bioscience). Briefly, 96-well ELISA plates were coated overnight at $4^{\circ} \mathrm{C}$ with a capture antibody, and excess antibody was removed after incubation, and the plates were blocked with 5\% or $10 \%$ FCS in PBS for $2 \mathrm{~h}$. The plates were subsequently washed three times with $0.05 \%$ tween in PBS, before being loaded with $50 \mu \mathrm{L}$ cell supernatant or standards. Plates were washed four times, followed by the addition of $50 \mu \mathrm{L}$ of detection antibody, before plates were incubated at room temperature for $1 \mathrm{~h}$, washed eight times, and then $100 \mu \mathrm{L}$ of $\mathrm{TMB}$ reagent (BD Bioscience) was added into each well. After colour development, the reaction was stopped with $100 \mu \mathrm{L}$ of stop solution, and absorbance was read at $460 \mathrm{~nm}$. 


\subsection{Results}

\subsubsection{Genome-wide analyses of aqueous $P$. insularum extract}

Genome-wide analyses using mutant libraries of yeast have been used to successfully identify MOA of compounds (Baetz et al., 2004; Carroll et al., 2009; Giaever et al., 1999; Giaever et al., 2004; Hillenmeyer et al., 2008; Lumet al., 2004; Parsons et al., 2004; Robinson et al., 2014; Smith et al., 2009). To determine the mechanism of action of the aqueous extract of $P$. insularum, we first identified a concentration to conduct the viability-based genome-wide analysis; this is a concentration that resulted in 10-20\% growth inhibition in the wild-type BY4741 strain. With a 10$20 \%$ inhibition in mutant strains, there will be a large window to detect growth inhibitions up to 80 $90 \%$ in the deletion strains. Via growth analysis of a representative library plate in 1,536 format, we conducted an image analysis using Gitter (Wagih \& Parts, 2014) and identified that 6\% v/v of the aqueous extract inhibited approximately $15 \%$ of yeast growth across the entire plate compared to agar vehicle control (Table 3.1)

Table 3.1: Optimization of aqueous extract concentration for genome-wide analysis. Colony sizes were measured using R-Gitter (arbitrary units) and residual growths were calculated by comparison to control median colony size.

\begin{tabular}{|c|c|ccc|}
\hline \multirow{2}{*}{} & & \multicolumn{3}{|c|}{ Aq. P. insularum } \\
\cline { 3 - 5 } & Control & $2 \% \mathrm{v} / \mathrm{v}$ & $4 \% \mathrm{v} / \mathrm{v}$ & $6 \% \mathrm{v} / \mathrm{v}$ \\
\hline Conoly size (median, AU) & 516.3 & 525.3 & 488.7 & 440.5 \\
Residual Growth (\%) & 100.0 & 101.7 & 94.6 & 85.3 \\
Standard Deviation (\%) & 3.1 & 6.2 & 4.3 & 3.5 \\
& & & & \\
\hline
\end{tabular}

We thus grew the $\sim 4,300$ haploid deletion strains in the deletion library ( $70 \%$ of the yeast genome) in the presence or absence of $6 \% \mathrm{v} / \mathrm{v}$ of the aqueous extract of $P$. insularum. Specifically, these strains were either grown on SC agar with the aqueous extract at $6 \% \mathrm{v} / \mathrm{v}$ or with $\mathrm{dH}_{2} \mathrm{O}$ at $6 \%$ v/v, in triplicate per treatment. After $24 \mathrm{~h}$, colony sizes were calculated using Gitter (Wagih \& Parts, 2014), from which growth ratios were calculated using ScreenMill (Dittmar et al., 2010). The growth ratio was a comparison of the colony size of each vehicle-treated deletion mutant against the colony 
size of each $P$. insularum-treated deletion mutant. Essentially, a growth ratio of 1 suggests the treated deletion mutant grew comparatively to its control counterpart. A growth ratio lower than 1 suggests the treated deletion mutant grew better than its control equivalent, while a growth ratio greater than 1 indicates the treated deletion mutant exhibited less growth compared to control. The majority of gene deletions exhibited a growth ratio between 0.8 and 1.2 (Fig 3.1) suggesting that the treatment with the aqueous extract of $P$. insularum did not drastically improve or reduce growth compared to growth under control conditions. There were 23 deletion mutants that exhibited a growth ratio less than 0.8 indicating the treatment with the aqueous extract of $P$. insularum improved their growth by at least $10 \%$ compared to control conditions. The increased growth of these gene deletions suggested that the deletion of the gene suppressed the effect of the extract. There were 88 gene deletions that exhibited a growth ratio greater than 1.2 , indicative of $10 \%$ growth inhibition of the deletion mutants in the presence of the aqueous extract of $P$. insularum. The hypersensitivity of these gene deletions suggested that these genes are required to buffer the effect of the $P$. insularum aqueous extract.

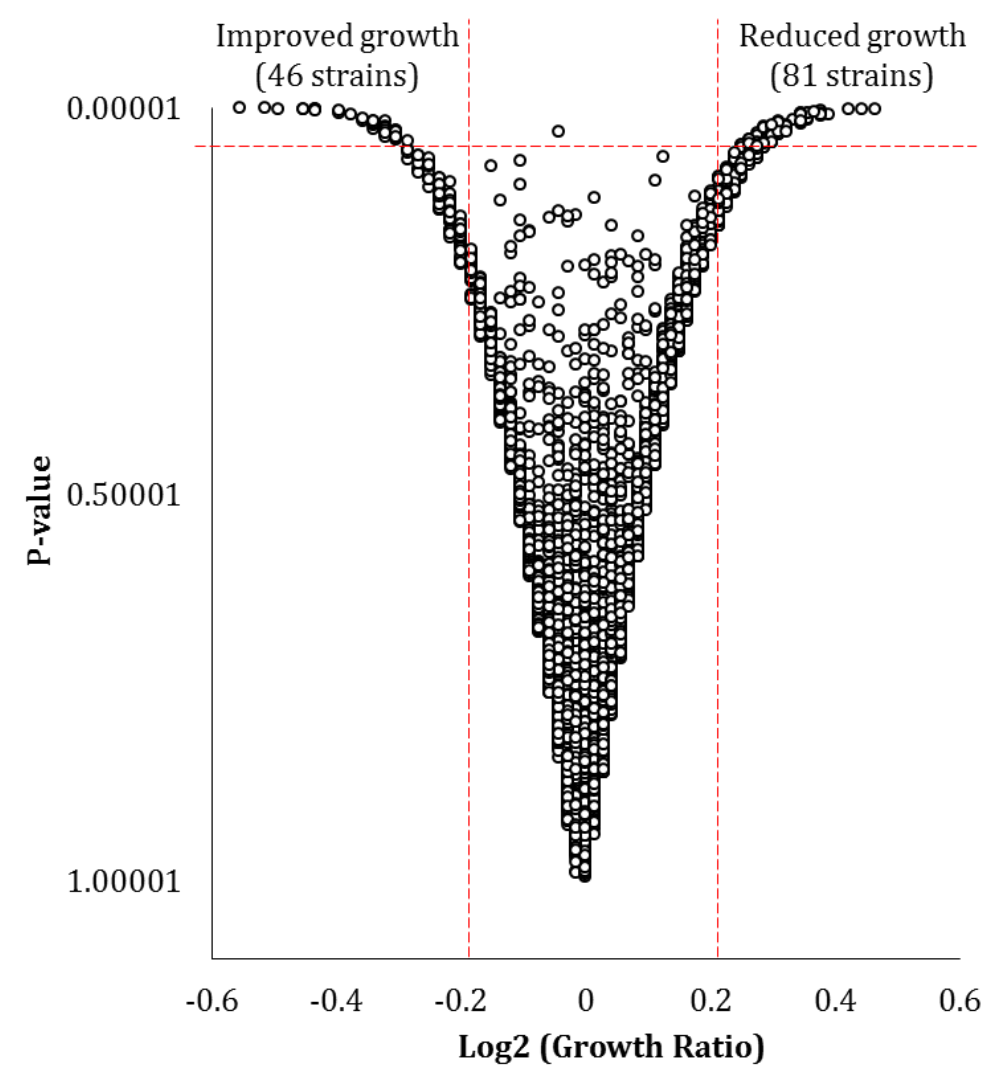


Figure 3.1: $\quad$ The scatterplot of gene deletion log2 growth ratio against aqueous $P$. insularum extract versus p-value. The haploid deletion mutant collection was analyzed against the aqueous extract of $P$. insularum extract in triplicate, and the growth of each deletion mutant was compared against its control counterpart. The average of the resulting fitness score across all three replicates was calculated and plotted as $\log 2$, against their corresponding p-values. The red-dashed lines mark the cut-off points of strains with improved and reduced growth, while the black-dashed line marks the p-value 0.05 . Based on this selection system, 46 strains had log2 growth ratio less than -0.2 and p-value less than 0.05 (improved growth) and 81 strains with $\log 2$ growth ratio greater than 0.2 and p-value less than 0.05 (reduced growth).

\subsubsection{Validations implicate various biological processes in P. insularum MOA}

Using the statistical evaluation of $p$-value cut-off of $<0.05$, we determined that the growth of 204,227 and 239 deletion mutants were significantly altered compared to the control, in replicates 1, 2 and 3 respectively. Across these 670 gene deletions, 128 mutants (Appendix IV) were present in two or more replicates. Sensitivity of these 128 gene deletion mutants was independently evaluated using a spot dilution assay. The gene deletion strains were grown in overnight cultures, and then serially diluted five-fold and inoculated on control agar or agar containing $2 \% \mathrm{v} / \mathrm{v}$ aqueous extract of $P$. insularum. The plates were visually analysed after $48 \mathrm{~h}$ of growth at $30^{\circ} \mathrm{C}$, and 29 of the 128 mutants were sensitive to the aqueous extract of $P$. insularum (Appendix V). Based on comparisons of growth at specific dilutions (red dashed circles in Fig 3.2), the most hypersensitive gene deletions to the aqueous $P$. insularum extract were bem $1 \Delta$, bem $4 \Delta, \operatorname{snf} 5 \Delta$, tef $4 \Delta$, and $y d r 269 c \Delta$. The BEM genes are involved in bud emergence. Specifically, BEM1 encodes a protein involved in establishing cell polarity and morphogenesis, while BEM4 encodes a protein involved in the establishment of cell polarity and bud emergence (Bender \& Pringle, 1991; Mack et al., 1996). SNF5 encodes a protein of the subunit of the SWI-SNF chromatin remodelling complex, while TEF4 encodes the gamma subunit of the eEF1B translational elongation factor (Kinzy et al., 1994; Peterson et al., 1994). YDR269C is a dubious open reading frame that overlaps CCC2 (Fig 3.3A), a copper transporter at the Golgi apparatus (Fisk et al., 2006; Fu et al., 1995). 


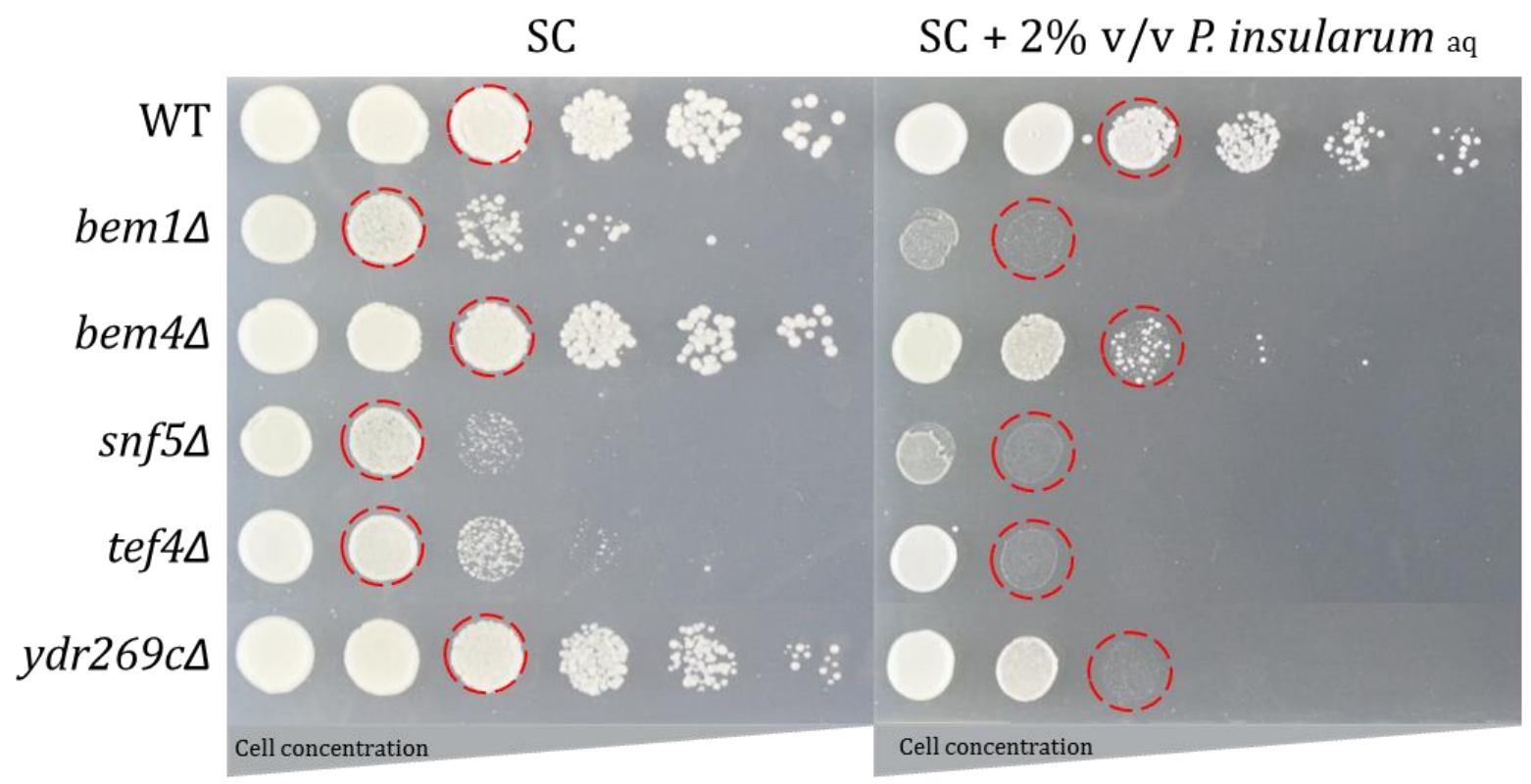

Figure 3.2: $\quad$ Spot dilution assay of five most hypersensitive gene deletions. Gene deletions were grown overnight then serially diluted, and subsequently spotted onto SC control agar, or SC agar containing $2 \% \mathrm{v} / \mathrm{v}$ aqueous extract of $P$. insularum( $P$. insularum ${ }_{\mathrm{aq}}$ ).Images for visual comparison were taken after $48 \mathrm{~h}$ growth at $30^{\circ} \mathrm{C}$. Gene deletions showing the most hypersensitivity to the aqueous extract of $P$. insularum are illustrated, including bem1 $1 \Delta$, bem $4 \Delta$, snf5 5 , tef $4 \Delta$ and $y d r 269 c \Delta$. Spots with red dashed circles indicate the circles that were compared against WT growth and treatment counterpart to illustrate degree of growth reduction.

\subsubsection{Deletion of $C C C 2$ is hypersensitive to the aqueous $P$. insularum extract}

As we determined above that $y d r 269 c \Delta$ is hypersensitive to the $P$. insularum aqueous extract (Fig 3.2) and that YDR269C is a dubious ORF that overlaps with CCC2 (Fig 3.3A), we sought to determine if $\operatorname{ccc} 2 \Delta$ was also hypersensitive to the $P$. insularum aqueous extract. WT, $y d r 269 c \Delta$ and $\operatorname{ccc} 2 \Delta$ were grown in increasing concentrations of $P$. insularum aqueous extract for $15 \mathrm{~h}$ at $30^{\circ} \mathrm{C}$. At concentrations less than $0.05 \% \mathrm{v} / \mathrm{v}$, there was not a dramatic difference in residual growth (treated/untreated) between the three strains; all strains exhibited residual growth values between $90-100 \%$ (0-10\% growth inhibition). In contrast, growth of $y d r 269 c \Delta$ and $\operatorname{ccc} 2 \Delta$ was significantly reduced at concentrations greater than $0.05 \% \mathrm{v} / \mathrm{v}$ compared to the WT, with residual growth values between $20-60 \%$ (40-80\% growth inhibition). These results suggest that the hypersensitivity of 
$y d r 269 c \Delta$ may be attributed to the deletion of nucleotides overlapping with $C C C 2$, particularly since YDR269C is a dubious ORF that does not encode a protein. These findings warranted further investigations into CCC2 and associated pathway(s).

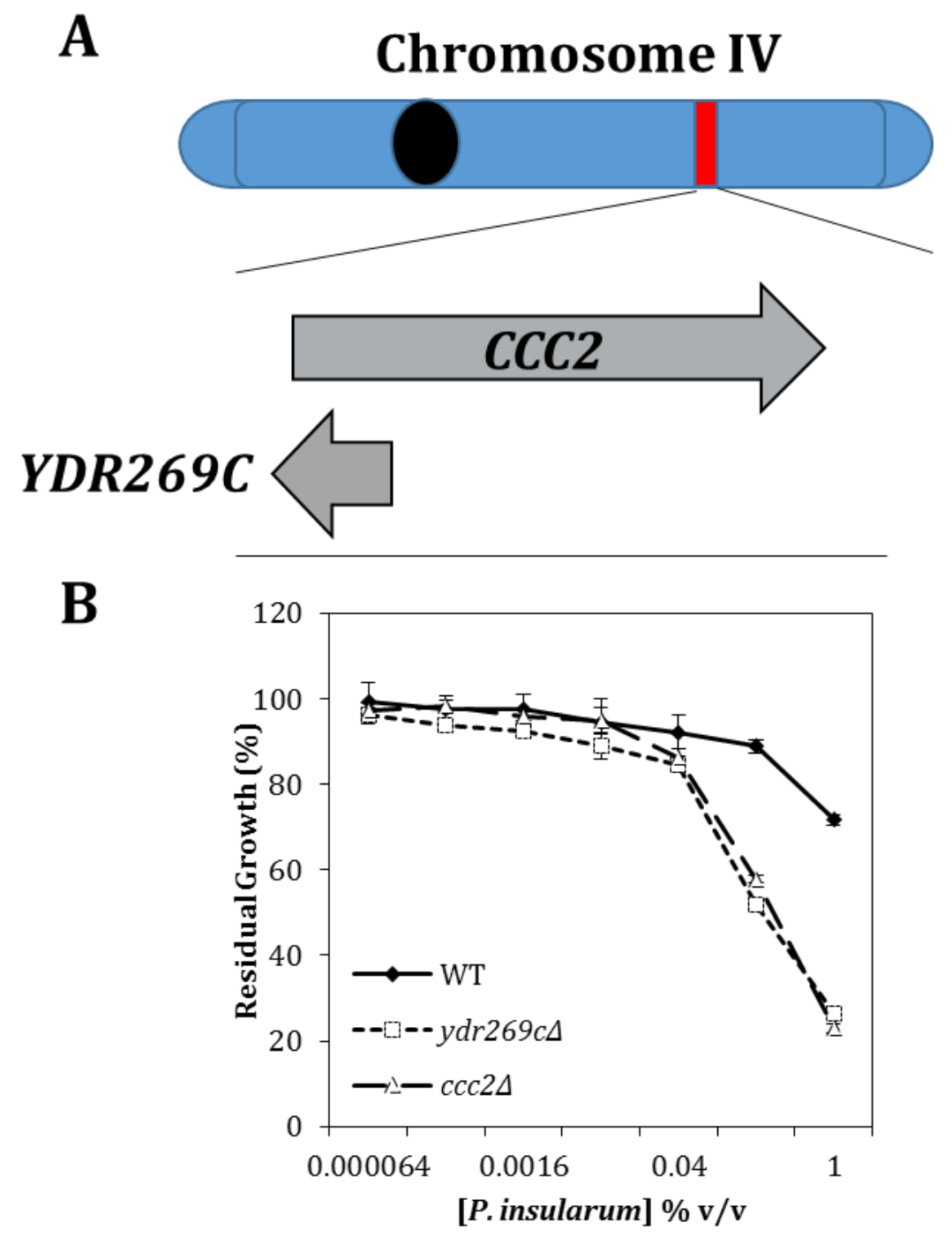

Figure 3.3: $\quad$ YDR269C and CCC2. A. The relationship between $Y D R 269 C$ and $C C C 2$, and how the two ORFs overlap. It is illustrated here that the deletion of YDR269C leads to the deletion of the starting sequence of CCC2. B: The residual growths of WT (solid black line), compared to the residual growths of $c c c 2 \Delta$ and $y d r 269 c \Delta$ (dashed black lines). WT and deletion strains were grown in the presence of increasing concentrations of the aqueous extract of $P$. insularum, at a concentration range of $6.4 \times 10^{-5}$ to $1 \% \mathrm{v} / \mathrm{v}$ with five-fold dilutions.

CCC2 encodes a copper transporter at the Golgi apparatus that is not under the control of the copper-sensing transcription factor Mac1p but is regulated by the iron-sensing transcription factor Aft1p. Indeed, CCC2 is primarily involved in iron transport into the cell (Yamaguchi-Iwai et al., 
1996). Iron transport in yeast occurs via three pathways: high-affinity iron transport, low-affinity iron transport, and an iron siderophore transport system (reviewed in Eide, 1998; van Ho et al., 2002). The high-affinity iron transport system involves the Fet3p-Ftr1p complex at the plasma membrane (Askwith et al., 1994; de Silva et al., 1995; Eide et al., 1994; Stearman et al., 1996). It is at this branch of iron transport that $\mathrm{Ccc} 2 \mathrm{p}$ is required as it functions to pump copper into the Golgi apparatus for its subsequent incorporation into Fet3p for its oxidase activity (Gaxiiola et al., 1998; Lin et al., 1997; Pufahl et al., 1997; Yuan et al., 1997). At the plasma membrane, Fet3p oxidase activity converts $\mathrm{Fe}^{2+}$ to $\mathrm{Fe}^{3+}$, which is successively transported into the cell by Ftr1p; this is specific as Ftr1p only transports $\mathrm{Fe}^{3+}$ from Fet3p (Askwith et al., 1994; de Silva et al., 1995; Stearman et al., 1996). The low-affinity iron transport system is composed solely of Fet4p, a non-specific metal transporter that pumps iron, zinc and copper into the cell (Dix et al., 1994; Dix et al., 1997; Hassett et al., 2000; Portnoy et al., 2001; Waters \& Eide, 2002). The iron siderophore transport system is composed of cell wall transporters (Fit1p, Fit2p, Fit3p and Fit4p) that scavenge iron siderophores, which are thenceforth taken into the cell via iron siderophore transporter vesicles (Arn1p, Arn2p, Arn3p and Arn4p) (Heymann et al., 2000; Philpott et al., 2002; Protchenko et al., 2001; Yuan et al., 2000). The involvement of $\operatorname{ccc} 2 p$ in iron transport necessitated an investigation of the iron transport systems and how it is affected by the aqueous $P$. insularum extract.

\subsubsection{Gene deletions of the iron transport systems are hypersensitive to the aqueous $P$. insularum extract}

To determine if the high-affinity iron transport system was sensitive to the aqueous extract of $P$. insularum, growth profiles of fet3 $\Delta$ and $f \operatorname{tr} 1 \Delta$ were compared to WT in the presence of the extract. The growth of $f e t 3 \Delta$ and $f t r 1 \Delta$ was reduced at concentrations greater than $0.05 \% \mathrm{v} / \mathrm{v}$ compared to WT with residual growth values between $10 \%-80 \%$ (20\%-90\% growth inhibition) (Fig 3.4A). To determine if the low-affinity iron transport system was sensitive to the aqueous extract of P. insularum, the growth profile of fet $4 \Delta$ was compared to WT in the presence of the extract. The 
growth profile of fet $4 \Delta$ also exhibited a prominent growth defect compared to WT at concentrations greater than $0.05 \% \mathrm{v} / \mathrm{v}$ with residual growth values between $80-10 \%$ (20\%-90\% growth inhibition). To determine if the iron siderophore transport system was sensitive to the aqueous extract of $P$. insularum, the growth profiles of $\operatorname{arn} 1 \Delta, \operatorname{arn} 2 \Delta, \operatorname{arn} 3 \Delta$ and $\operatorname{arn} 4 \Delta$ as well as fit1 $\Delta$, fit $2 \Delta$, fit $3 \Delta$ and fit $4 \Delta$ were compared to WT in the presence of the extract. All six representative gene deletions of the iron siderophore transport system were also all shown to be hypersensitive to the aqueous extract of $P$. insularum in comparison to WT at concentrations greater than $0.05 \% \mathrm{v} / \mathrm{v}$ with residual growth values between $80-10 \%$ (20\%-90\% growth inhibition). Although all gene deletions of the iron transport systems showed varying degrees of sensitivity to the aqueous extract of $P$. insularum, all exhibited pronounced growth defects compared to WT, suggesting each of these genes are required to buffer the effects of the extract.
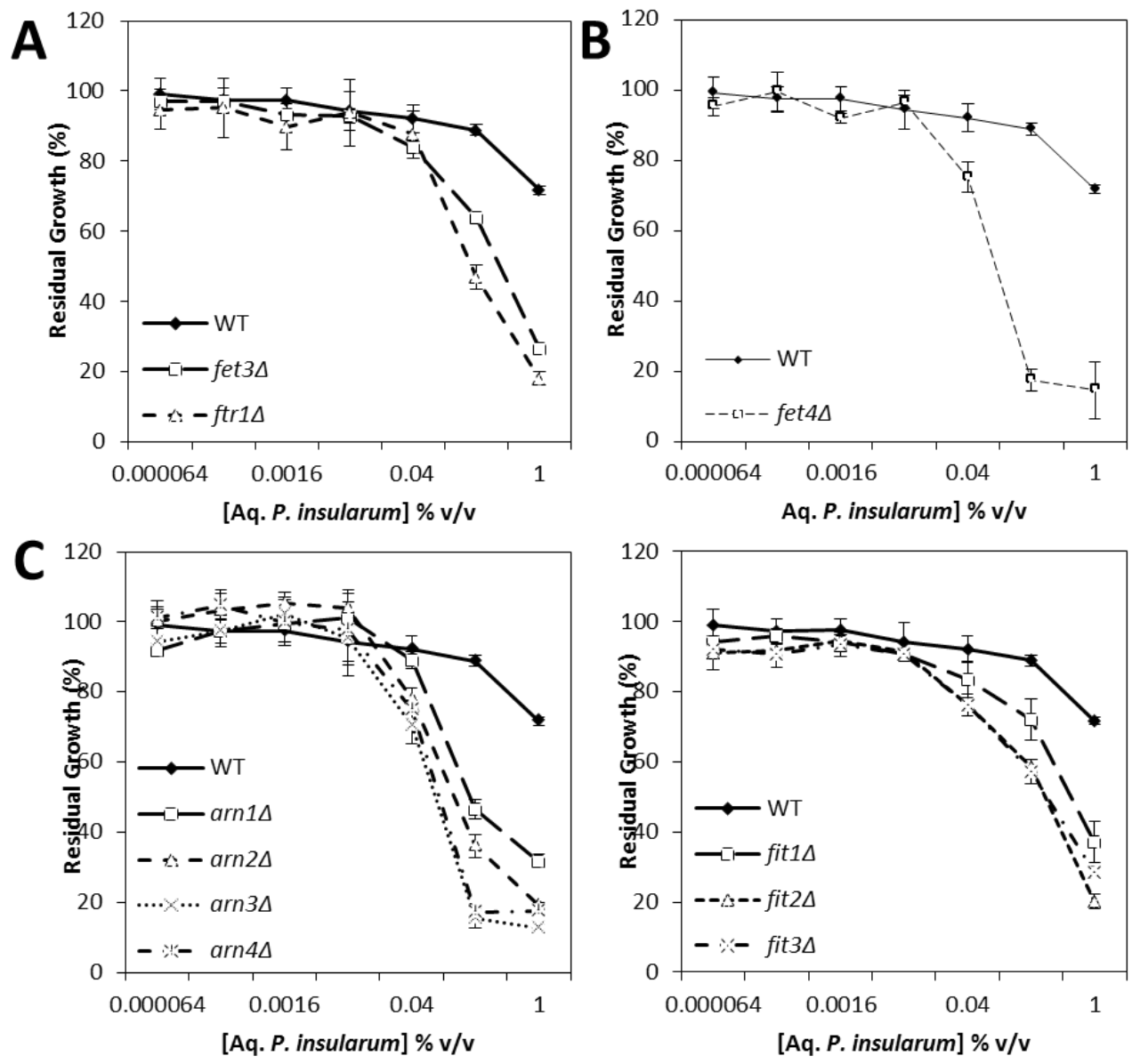
Figure 3.4: Growth profiles of gene deletions from the iron transport systems against the aqueous extract of $\boldsymbol{P}$. insularum. Liquid based assays were carried out using the aqueous extract of $P$. insularum over increasing concentrations from $6.4 \times 10^{-5}$ to $1 \% \mathrm{v} / \mathrm{v}$ with five-fold dilutions. Absorbance readings at $590 \mathrm{~nm}$ after $15 \mathrm{~h}$ growth at $30^{\circ} \mathrm{C}$ compared to their control treated counterparts were used to calculate residual growths. A: Residual growth comparison of WT to gene deletions of the high affinity iron transport system, fet $3 \Delta$ and ftr1 $\Delta$. B: Residual growth comparison of WT to gene deletions of the low affinity iron transport system fet4A. C: Residual growth comparison of WT to gene deletions of the iron

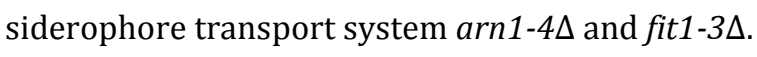

\subsubsection{Gene deletions of the iron transport systems are hypersensitive to the methanolic P. insularum extract}

On the principle that the bioactivity of crude extracts are often accounted for by a single bioactive compound or a single class of bioactive compounds such as peloruside A and B which are antitumor compounds that promote microtubule polymerisation and isolated from Mycale hentscheli (Singh et al., 2010), we proposed that the methanolic extract, which was shown to be bioactive in Chapter 2 (Figs $2.6 \& 2.7$ ), may follow a similar mechanism as that of the $P$. insularum aqueous extract. To determine if this was the case, the growth profiles of gene deletion strains involved in the high affinity transport system including the initially detected deletions of YDR269C and $\operatorname{CCC} 2(y d r 269 c \Delta, \operatorname{ccc} 2 \Delta, f e t 3 \Delta$ and $f \operatorname{tr} 1 \Delta)$, the low affinity iron transport system $(f e t 4 \Delta)$ and the iron siderophore transport systems $(\operatorname{arn} 1 \Delta, \operatorname{arn} 2 \Delta, \operatorname{arn} 3 \Delta, \operatorname{arn} 4 \Delta$ and fit1 $\Delta$, fit2 $\Delta$ and fit3 $\Delta$ ) were monitored over a range of concentration of the methanolic extract $\left(6.4 \times 10^{-5}\right.$ to $\left.1 \% \mathrm{v} / \mathrm{v}\right)$ relative to a WT control. Similar to their growth profiles in the presence of the aqueous extract of $P$. insularum, the gene deletions of the three iron transport systems all exhibited hypersensitivity to the methanolic extract indicated by their more pronounced growth defect compared to WT (Fig 3.5). Gene deletions of the high affinity iron transport system and those from the iron siderophore transport systems exhibited residual growth values of $80 \%-20 \%$ (20\%-80\% growth inhibition) at concentrations greater than $0.005 \% \mathrm{v} / \mathrm{v}$ compared to WT residual growth. The gene deletion of the low-affinity iron transport system $(f e t 4 \Delta)$ showed residual growth ranging from $80 \%-20 \%$ (20\%-80\% 
growth inhibition) at concentrations greater than $0.0005 \% \mathrm{v} / \mathrm{v}$ compared to WT. These results suggest that both the aqueous and methanolic extracts of $P$. insularum mediate their bioactivity via a similar mechanism involving iron transport. Although it was observed that all iron transporters tested were hypersensitive to the extracts of $P$. insularum compared to WT, it was unlikely that this was a general metal transporter phenotype, as gene deletions of zinc transporters did not exhibit hypersensitivity (data not shown).
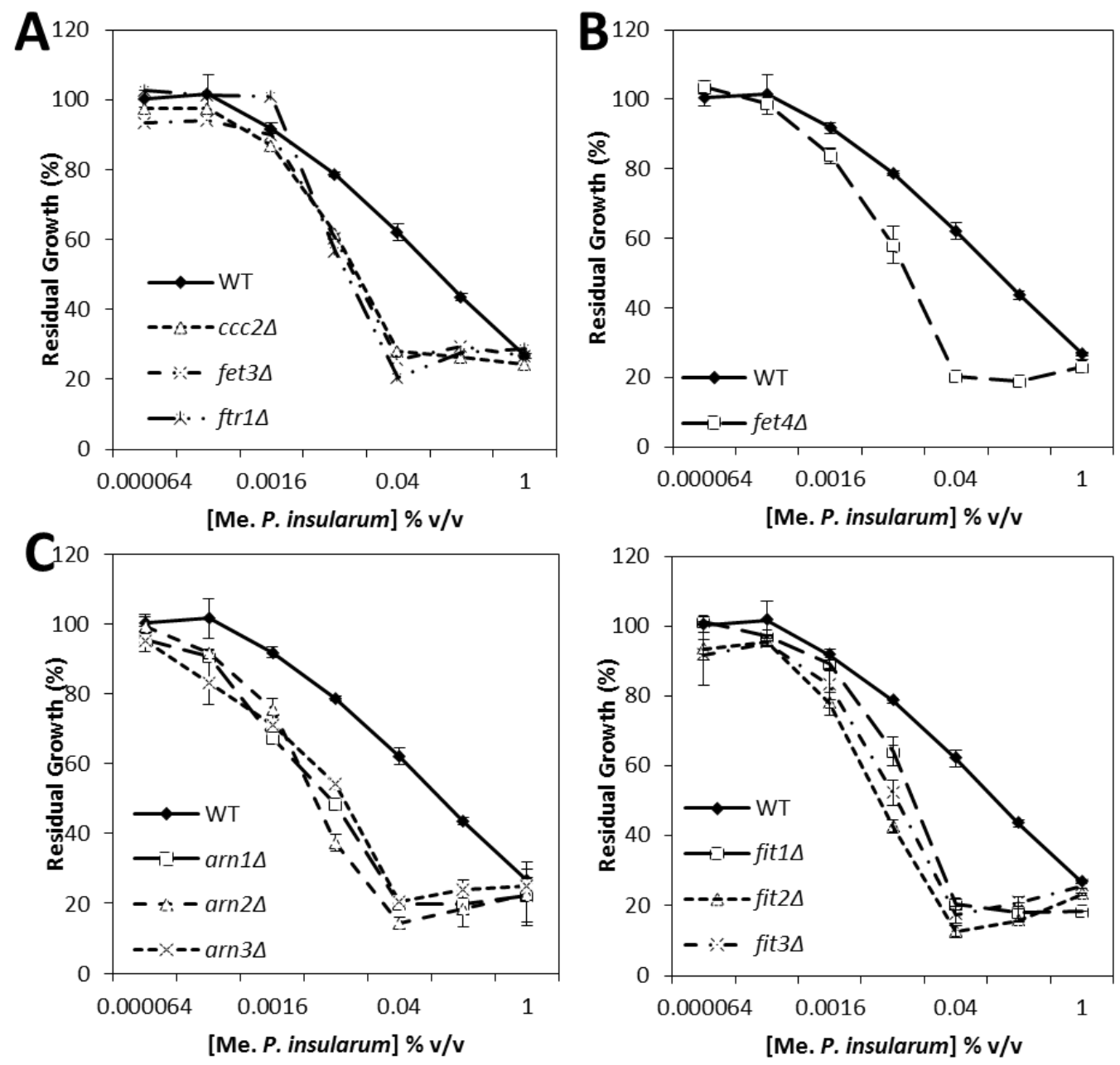

Figure 3.5: Growth profiles of gene deletions from the iron transport systems against the methanolic extract of $\boldsymbol{P}$. insularum. Liquid-based growth assays were carried out using the methanolic extract of $P$. insularum over increasing concentrations from of $6.4 \times 10^{-5}$ to $1 \% \mathrm{v} / \mathrm{v}$ with five-fold dilutions. Absorbance readings at $590 \mathrm{~nm}$ after $15 \mathrm{~h}$ growth at $30^{\circ} \mathrm{C}$ compared to their control treated counterparts were used to calculate residual growths A: Residual growth comparison of WT to gene deletions of the high affinity iron transport system, $y d r 269 c \Delta, c c c 2 \Delta$,fet $3 \Delta$ and $f t r 1 \Delta$. B: Residual growth comparison of WT to gene deletion of the low affinity iron transporter fet $4 \Delta$. C: Residual growth comparison of WT to

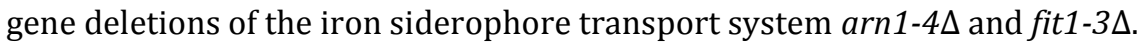




\subsubsection{Iron supplementation rescues the growth defect induced by aqueous $P$. insularum extract}

The hypersensitivity of gene deletions of the iron transport systems to the aqueous and methanolic extracts of $P$. insularum suggests that the iron transport genes are required to buffer the effects of the $P$. insularum extracts. Because the genes function to provide iron to the cell and their deletions led to hypersensitivity, we hypothesized the addition of iron will reduce the growth defect induced by extract treatment. To test this hypothesis, three sets of liquid-based assays using BY4741 WT were carried out with increasing concentrations of either the aqueous or methanolic extract of P. insularum, with supplementation of either $100 \mu \mathrm{M} \mathrm{FeCl}, 100 \mu \mathrm{M} \mathrm{FeSO}_{4}$, or no supplement at all. Supplementation with $\mathrm{FeCl}_{3}$ and $\mathrm{FeSO}_{4}$ ensured that both forms of iron (ferric and ferrous respectively) were investigated, each at the previously established $100 \mu \mathrm{M}$ iron concentration for optimal yeast growth (Shakoury-Elizeh et al., 2010). The BY4741 WT used here showed $70 \%-10 \%$ residual growth (30\%-90\% growth inhibition) at aqueous extract concentrations greater than $0.005 \%$ v/v (Fig 3.6A). On the other hand, BY4741 WT grown in the presence of the methanolic $P$. insularum extract exhibited residual growth values ranging from $70 \%-10 \%$ (30\%-90\% growth inhibition) at concentrations greater than $0.0001 \% \mathrm{v} / \mathrm{v}$ (Fig 3.6B). Supplementation with $100 \mu \mathrm{M} \mathrm{FeCl}{ }_{3}$ resulted in a shift of growth of WT in media containing the aqueous extract, indicating $\mathrm{FeCl}_{3}$ reduced the aqueous extract treatment-induced growth defect by $80 \%$ at $0.2 \% \mathrm{v} / \mathrm{v}$, and by $50 \%$ at $1 \% \mathrm{v} / \mathrm{v}$. Similarly, the growth profile of WT cells grown in the aqueous extract of $P$. insularum supplemented with $100 \mu \mathrm{M}$ of $\mathrm{FeSO}_{4}$ also exhibited a shift, however, it did not match the shift observed from $\mathrm{FeCl}_{3}$. For instance, $100 \mu \mathrm{M} \mathrm{FeCl}_{3}$ was able to rescue WT growth from $20 \%$ residual growth to $100 \%$ residual growth at $0.2 \% \mathrm{v} / \mathrm{v}$ aqueous extract treatment, while $\mathrm{FeSO}_{4}$ was only able to rescue to approximately $70 \%$ residual growth (Fig 3.6A, dashed lines). Likewise, $\mathrm{FeCl}_{3}$ and $\mathrm{FeSO}_{4}$ supplementation variable rescued the methanolic extract treatment-induced growth defect. Specifically, $100 \mu \mathrm{M} \mathrm{FeCl}$ fully rescued growth of WT (from $80 \%$ growth inhibition to $0 \%$ growth 
inhibition) from treatment with $0.008 \% \mathrm{v} / \mathrm{v}$ of methanolic extract, while $100 \mu \mathrm{M} \mathrm{FeSO}_{4}$ supplementation partially rescued growth of WT ( $80 \%$ growth inhibition to $20 \%$ growth inhibition) (Fig 3.6B). These results suggest that supplementation of extract treatment with exogenous iron reduced the effect of the extract and improved yeast growth. Further, these findings showed that ferric iron $\left(\mathrm{FeCl}_{3}\right)$ rescued yeast growth slightly better than ferrous iron $\left(\mathrm{FeSO}_{4}\right)$.

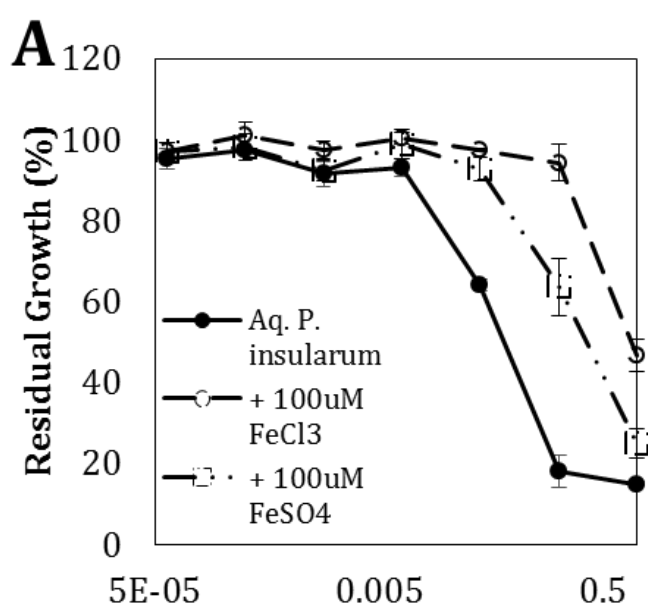

[Aq. P. insularum] \% v/v

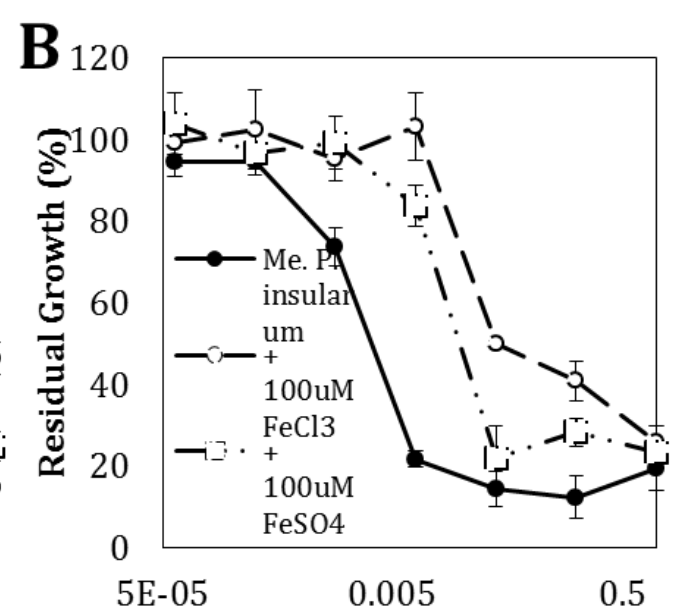

[Me. P. insularum] \% v/v

Figure 3.6: $\quad$ Iron supplementation against aqueous and methanolic extracts of $\boldsymbol{P}$. insularum. Liquid-based growth assays of BY4741 WT were prepared as previously described with increasing concentrations of extract without iron supplementation, compared to metal supplemented extract treatments with $100 \mu \mathrm{M}$ of the relevant metal salt solution. A: Growth profiles of BY4741 WT in the presence of aqueous $P$. insularum extract (solid line) without iron supplementation, with $100 \mu \mathrm{M} \mathrm{FeCl}_{3}$ (dashed line with circle) or with $100 \mu \mathrm{M} \mathrm{FeSO}_{4}$ (dashed line with square) B: Growth profiles of BY4741 WT in the presence of methanol $P$. insularum extract without iron supplementation (solid line), with 100 $\mu \mathrm{M} \mathrm{FeCl}$ (dashed line with circle) or with $100 \mu \mathrm{M} \mathrm{FeSO}_{4}$ (dashed line with square).

3.3.7 Iron rescue is specific to P. insularum extract treatment and is not a cytoprotective effect of iron supplementation

Iron has been shown to contribute to drug susceptibility and resistance (Prasad et al., 2006) suggesting that iron may exhibit a cytoprotective effect against a drug treatment independent of the drug's MOA. To investigate if the cytoprotective phenomenon of iron in suppressing sensitivity of WT cells to P. insularum extracts (Fig 3.6) is conserved for other drugs, BY4741 WT 
yeast cells were grown in the presence of two well characterized drugs, cycloheximide (which disrupts translation, Schneider-Poestch et al., 2010) and atorvastatin (which targets ergosterol

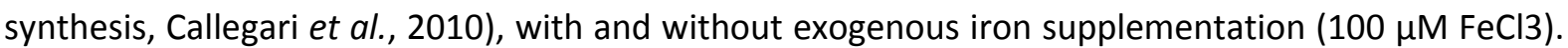
Whilst BY4741 demonstrated sensitivity to atorvastatin at increasing concentrations, no difference was observed between BY4741 growth in drug alone compared to the drug treatment with iron supplementation. For example, aqueous $P$. insularum extract treatment of WT with $0.2 \% \mathrm{v} / \mathrm{v}$ caused an $80 \%$ growth inhibition and supplementation with $100 \mu \mathrm{M} \mathrm{FeCl}$ diminished the extract-induced growth inhibition, however, iron supplementation of atorvastatin treatment (Fig 3.7A) or cycloheximide treatment (Fig 3.7B) failed to rescue even a growth inhibition of $20 \%$. These findings demonstrate that iron supplementation did not rescue the growth defects induced by atorvastatin or cycloheximide. Subsequently, these results indicate that the rescue observed from iron supplementation in $P$. insularum extract treatments was unlikely a consequence of iron playing a general cytoprotective role, but rather a specific function relating to the MOA of the $P$. insularum extracts.
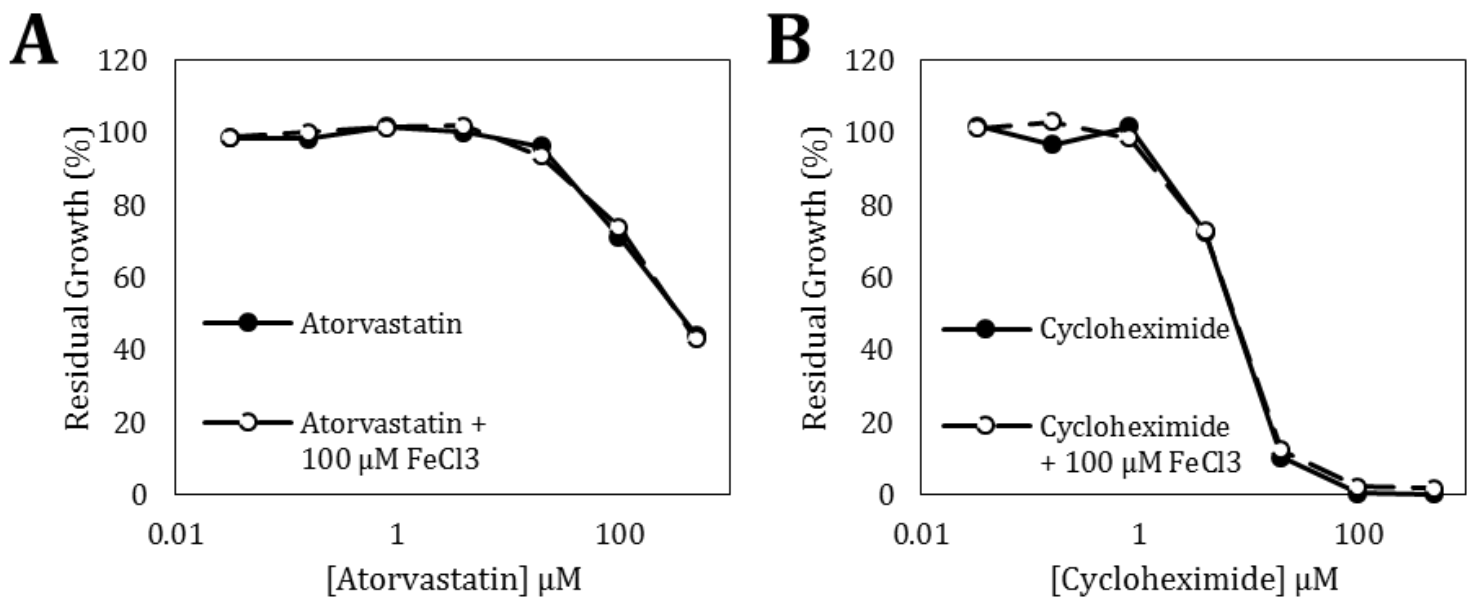

Figure 3.7: Iron supplementation of atorvastatin and cycloheximide drug treatment of BY4741 WT yeast. A: BY4741 WT treatment with atorvastatin. B: BY4741 WT treatment with cycloheximide. BY4741 cells were inoculated into SC media at $5 \times 10^{5} \mathrm{cells} / \mathrm{mL}$ and treated at increasing concentrations of drug, or drug with $100 \mu \mathrm{M} \mathrm{FeCl}_{3}$ supplementation. Residual growths were calculated by comparison to the control. Solid lines indicate treatment with drug alone, dashed lines correspond to treatment with drug and iron supplementation. 


\section{P. insularum extracts in a Fet3p-dependent manner}

Copper is required in the high affinity iron transport pathway for the oxidase activity of Fet3p (Askwith et al., 1994). We thus hypothesized that the addition of copper to P. insularum extract treatments may also rescue the extract-induced growth defect. To test this hypothesis, both WT and fet3 $\Delta$ yeast strains were grown in the presence of both aqueous and methanolic extracts of P. insularum, with or without the presence of copper supplementation $\left(100 \mu \mathrm{M} \mathrm{CuCl}_{2}\right)$. Supplementation with $\mathrm{CuCl}_{2}$ modestly reduced the growth defect resulting from aqueous extract treatment (Fig 3.8A). For instance, at $0.2 \% \mathrm{v} / \mathrm{v}$ extract treatment, copper supplementation was only able to rescue growth from $20 \%$ to $40 \%$ residual growth while $\mathrm{FeCl}_{3}$ was able to completely rescue at this concentration from $20 \%$ to $100 \%$ residual growth. A stronger rescue was observed from copper supplementation of WT treatment with the methanolic extract of $P$. insularum (Fig 3.8B). Similar to $\mathrm{FeCl}_{3}$ rescue, $\mathrm{CuCl}_{2}$ supplementation was able to rescue the growth defect induced by $0.008 \% \mathrm{v} / \mathrm{v}$ treatment with methanolic extract from $20 \%$ residual growth to $80 \%$. Contrastingly, $\mathrm{CuCl}_{2}$ supplementation failed to reduce the effect of the aqueous extract on fet3 $\Delta$ growth, while $\mathrm{FeCl}_{3}$ supplementation rescued the fet $3 \Delta$ growth defect resulting from treatment with the aqueous extract of $P$. insularum (Fig $3.8 \mathrm{C}$ ). A similar result was obtained from fet $3 \Delta$ treated with the methanolic extract of $P$. insularum, whereby supplementation with $\mathrm{CuCl}_{2}$ did not clearly exhibit a rescue phenotype, while supplementation with $\mathrm{FeCl}_{3}$ reduced the growth defect induced by the methanolic extract (Fig 3.8D). These findings suggest that the moderate rescue observed from copper supplementation in BY4741 WT but not fet $3 \Delta$ was mediated through the copper-dependent oxidase activity of the high-affinity iron transporter Fet3p, in the case of the aqueous extract of $P$. insularum. Further, the greater rescue observed from $\mathrm{CuCl}_{2}$ supplementation of the methanolic extract compared to the aqueous extract suggests the methanolic extract component responsible for the iron transport-related phenotype may also affect copper transport in yeast cells, given that the deletion of FET3 did not completely remove the copper rescue. 

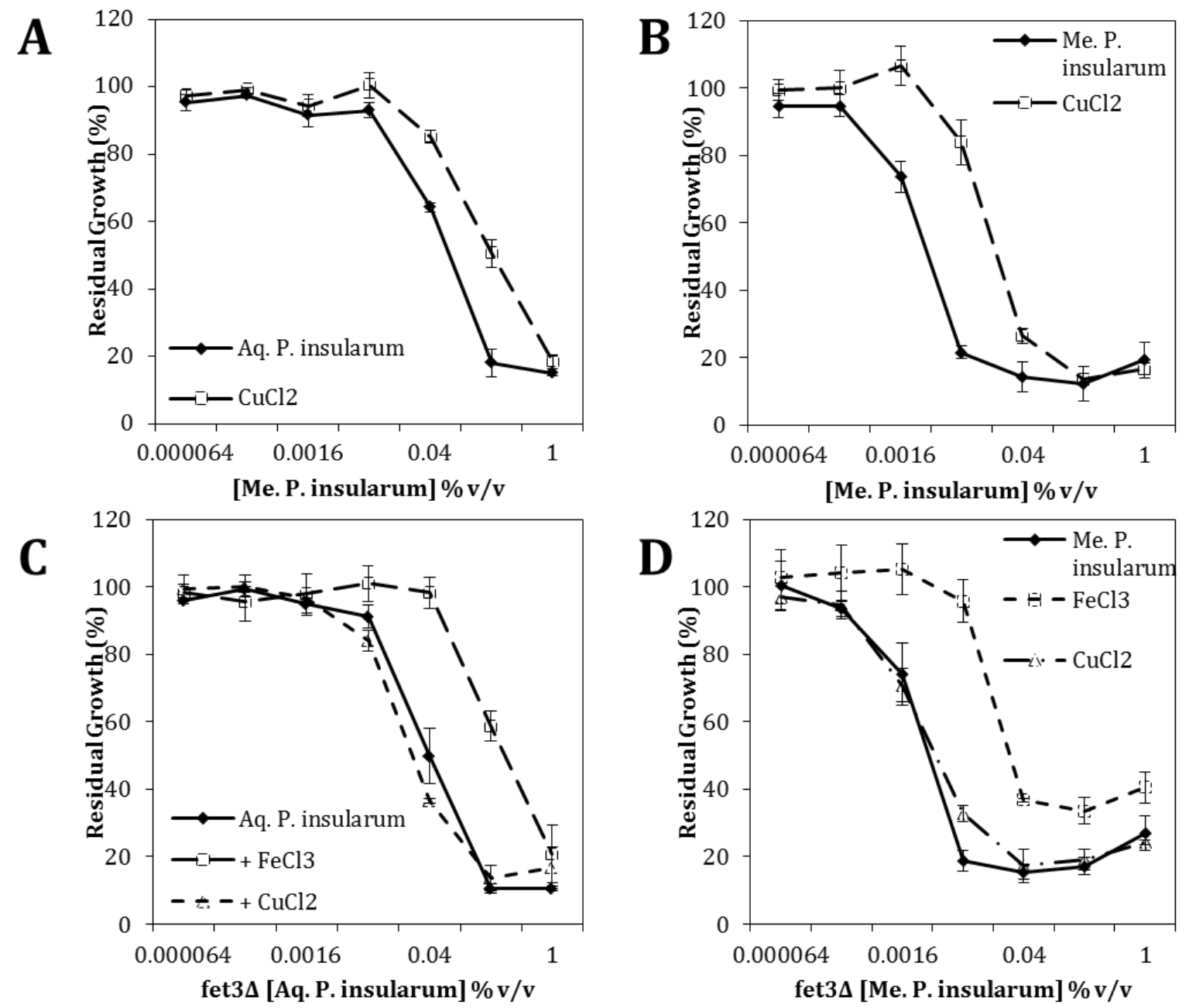

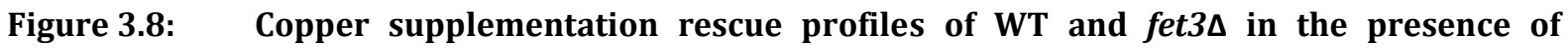
aqueous and methanolic extracts of $\boldsymbol{P}$. insularum. Liquid-based growth assays of BY4741 WT cells in increasing concentrations of extract, compared to metal supplemented extract treatments with $100 \mu \mathrm{M}$ of the relevant metal salt solution. A: Comparison of WT growth profiles in the presence of aqueous $P$. insularum extract (solid black line), and exogenous copper supplementation (dashed line). B: Comparison of WT growth profiles in the presence of methanolic P. insularum extract (solid black line) and WT growth with exogenous copper supplementation (dashed line). C: Growth profiles of fet $3 \Delta$ deletion mutant grown in the presence of aqueous $P$. insularum extract (solid black line), with exogenous $\mathrm{FeCl}_{3}$ supplementation (dashed line with sqaure markers) or copper supplementation (dashed lines with triangle markers). D: Growth profiles of fet $3 \Delta$ deletion mutant grown in the presence of methanolic P. insularum extract (solid black line), with exogenous $\mathrm{FeCl}_{3}$ supplementation (dashed line with square markers), or copper supplementation (dashed lines with triangle markers). 
3.3.9 Supplementation with other metals does not rescue the growth defect induced by extracts of $P$. insularum

Given that iron and copper supplementation rescued WT cells from the activity of $P$. insularum extracts, it was obligatory to investigate the effects of other metals on the activity of $P$. insularum extracts. This was achieved through growth assays of WT in the presence of either the aqueous or methanolic extracts of $P$. insularum supplemented with $100 \mu \mathrm{M} \mathrm{CaCl}_{2}, \mathrm{MgCl}_{2}, \mathrm{MnCl}_{2} \mathrm{Or}$ $\mathrm{ZnCl}_{2}$. Supplementation with $\mathrm{CaCl}_{2}, \mathrm{MgCl}_{2}, \mathrm{MnCl}_{2}$ or $\mathrm{ZnCl}_{2}$ did not exhibit any significant effects on aqueous extract activity, as the growth profiles of BY4741 treated with aqueous extract alone followed the same growth pattern as those with metal supplementation (Fig 3.9A). The same lack of difference was observed from BY4741 grown in the methanolic extract of $P$. insularum, suggesting that calcium, magnesium, manganese and zinc do not exert any effects on the activity of $P$. insularum extracts, unlike iron and copper.
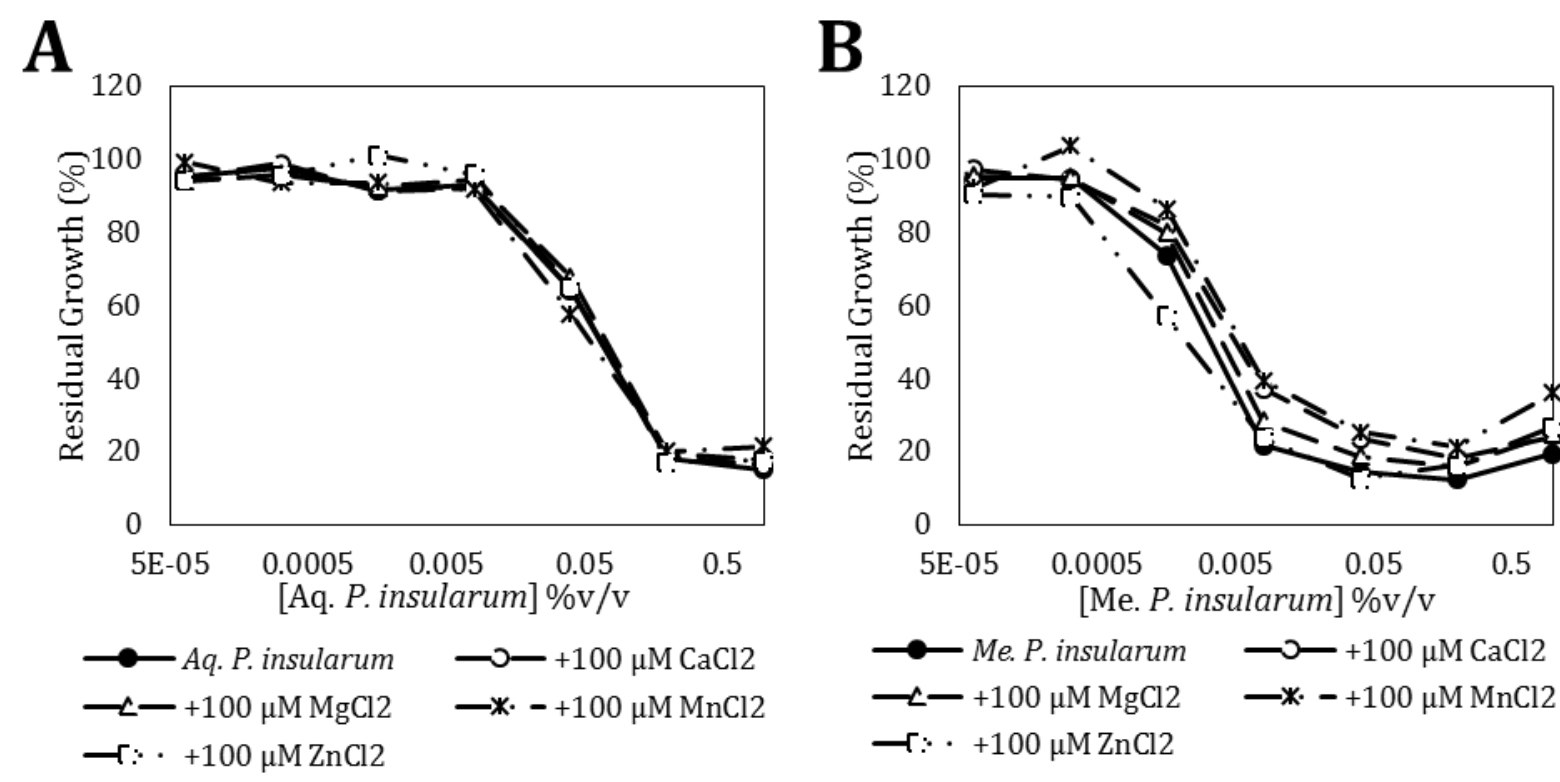

Figure 3.9: $\quad$ Growth profiles of WT against aqueous and methanolic extracts of $P$. insularum with metal supplementation. Liquid-based growth assays of BY4741 WT cells in increasing concentrations of extract, compared to metal supplemented extract treatments with $100 \mu \mathrm{M}$ of the relevant metal salt solution. A: Metal supplementation of WT grown in the aqueous extract of $P$. insularum. B: Metal supplementation of WT grown in the methanol extract of $P$. insularum. Extract treatment (solid line) and metal supplementation (dashed lines) of $\mathrm{CaCl}_{2}$ (circle marker), $\mathrm{MgCl}_{2}$ (triangle marker), $\mathrm{MnCl}_{2}$ (asterisk marker) and $\mathrm{ZnCl}_{2}$ (square marker). 


\subsubsection{Intracellular iron is decreased in the presence of $P$. insularum extracts}

Iron supplementation rescued the growth defect induced by the extract of $P$. insularum (Fig 3.6), implying that the treatment of cells with the extracts reduced intracellular iron. To determine if this was the case, inductively coupled plasma mass spectrometry (ICP-MS) was used to quantify intracellular iron in WT cells treated with the extracts of $P$. insularum. For comparison, cells were also grown in iron-free media, or treated with the iron chelator bathophenanthroline disulfonic acid (BPS). Treatment of WT BY4741 cells led to an approximate 50\% reduction in growth of cells treated with the aqueous $P$. insularum extract, the methanolic $P$. insularum extract or the iron chelator compared to control cells (Fig 3.10). Additionally, cells grown in iron-free media showed an approximate $80 \%$ reduction in total intracellular iron compared to the control cells. These results indicate that the $P$. insularum extracts chelate iron as effectively as an established iron chelator.

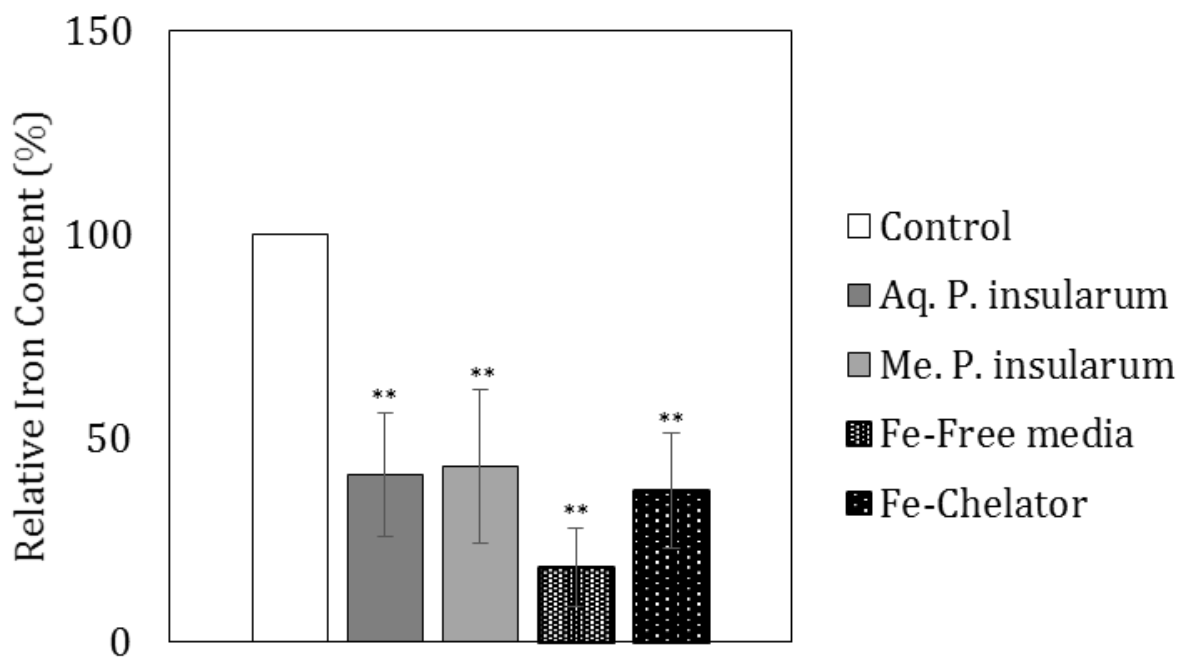

Figure 3.10: Comparison of relative intracellular iron between control and treated WT. BY4741 cells were grown in control media (Control), as well as in treatment media containing $0.05 \% \mathrm{v} / \mathrm{v}$ of aqueous extract (Aq. P. insularum) and $0.005 \%$ v/v of methanol extract (Me. P. insularum), as well as ironfree media (Fe-free media) and media containing $0.1 \mu \mathrm{M}$ bathophenanthroline disulfonic acid (FeChelator). Quantified iron is reported as relative total intracellular iron, compared to control BY4741, obtained from the average and standard deviation of two biological replicates (**: p-value $<0.01)$. 


\subsubsection{P. insularum extracts up-regulate the expression of iron transporters}

To determine if the expression of iron transporters is affected by extract treatment, the fluorescence of GFP-tagged iron transporters was quantified using fluorescent microscopy. A special feature of the GFP strains utilized here was the incorporation of an mCherry nuclear localising signal, a high intensity red fluorescence tag, and a RedStar2 cytoplasmic signal, a low intensity red fluorescence tag (Bircham et al., 2011), two signals that identified the nucleus and the cell periphery, allowing the quantification of GFP signal per cell per treatment relative to control. In these experiments, the green fluorescence intensity was an indicator of the protein expression of the iron transporters which the GFP tag was attached to. Contrary to the reduced intracellular iron content of extract-treated cells (Fig 3.10), proteins representative of the three iron transporter systems were significantly increased compared to control cells, when the green fluorescence intensity was assessed visually (Fig 3.11), and quantified(Fig 3.12).

Fet3p and Ftr1p were chosen to represent the high-affinity transport system. The fluorescence of GFP tagged onto both Fet3p and Ftr1p increased when the strains were treated with the extracts of $P$. insularum, the iron-chelator and when grown in iron-free media. Indeed, when quantified, the aqueous extract treatment induced a significant $50 \%$ increase in Fet3p-GFP fluorescence, while methanolic extract treatment caused a significant $30 \%$ increase compared to the control. Fet3p-GFP cells grown in iron-free media resulted in a significant $100 \%$ increase in GFP intensity, while the iron chelator treatment induced a significant $70 \%$ increase. The aqueous extract treatment of Ftr1p-GFP showed a modest but significant 30\% increases in GFP fluorescence. Both iron-free media and iron chelator treatments resulted in $50 \%$ and $40 \%$ increases in GFP fluorescence, respectively.

Fet $4 p$ represented the low-affinity iron transport system. The fluorescence of GFP tagged onto Fet $4 p$ increased when the strain was treated with the extracts of $P$. insularum, the ironchelator and when grown in iron-free media. This was statistically quantified to a $30 \%$ increase when 
treated with $P$. insularum extracts. When grown in iron-free media, a significant $80 \%$ increase in GFP fluorescence was seen, while growth in iron chelator treatment induced a significant $60 \%$ increase.

Arn1p and Arn3p were chosen to represent the iron siderophore transport system. The fluorescence of GFP tagged onto both Arn1p and Arn3p increased when the strains were treated with the extracts of $P$. insularum, the iron-chelator and when grown in iron-free media. When quantified, Arn1p-GFP showed a significant $30 \%$ increase in fluorescence intensity when treated with the aqueous extract of $P$. insularum. However, the $10 \%$ increase observed from the methanolic extract treatment was not statistically significant. Arn1p-GFP exhibited an extraordinary and significant $180 \%$ increase in fluorescence when grown in iron-free media, while a significant $50 \%$ increase was observed when grown in the presence of the iron chelator. Arn3p-GFP showed modest yet not significant $12-15 \%$ increases in GFP intensity from aqueous and methanolic extract treatments. Significant and dramatic increases of $80 \%$ and $50 \%$ in Arn1p-GFP fluorescence were observed in cells grown in iron-free media and the iron chelator, respectively.

Intriguingly, the increased expression of proteins representative of the three iron transport systems in extract-treated cells was significantly reduced to control levels with exogenous iron supplementation. These findings thus reveal that yeast cells, specifically iron transport proteins, respond to $P$. insularum extract in a comparable manner to responding to iron-free media or the presence of an iron chelator. The cells increase expression of the iron transporters as a cellular response to low iron, further supporting our hypothesis that there is low available iron in the cell as a consequence of $P$. insularum extract treatment. 
High Affinity

Iron

Transport
Low Affinity

Iron

Transport
Iron

Siderophore

Transport

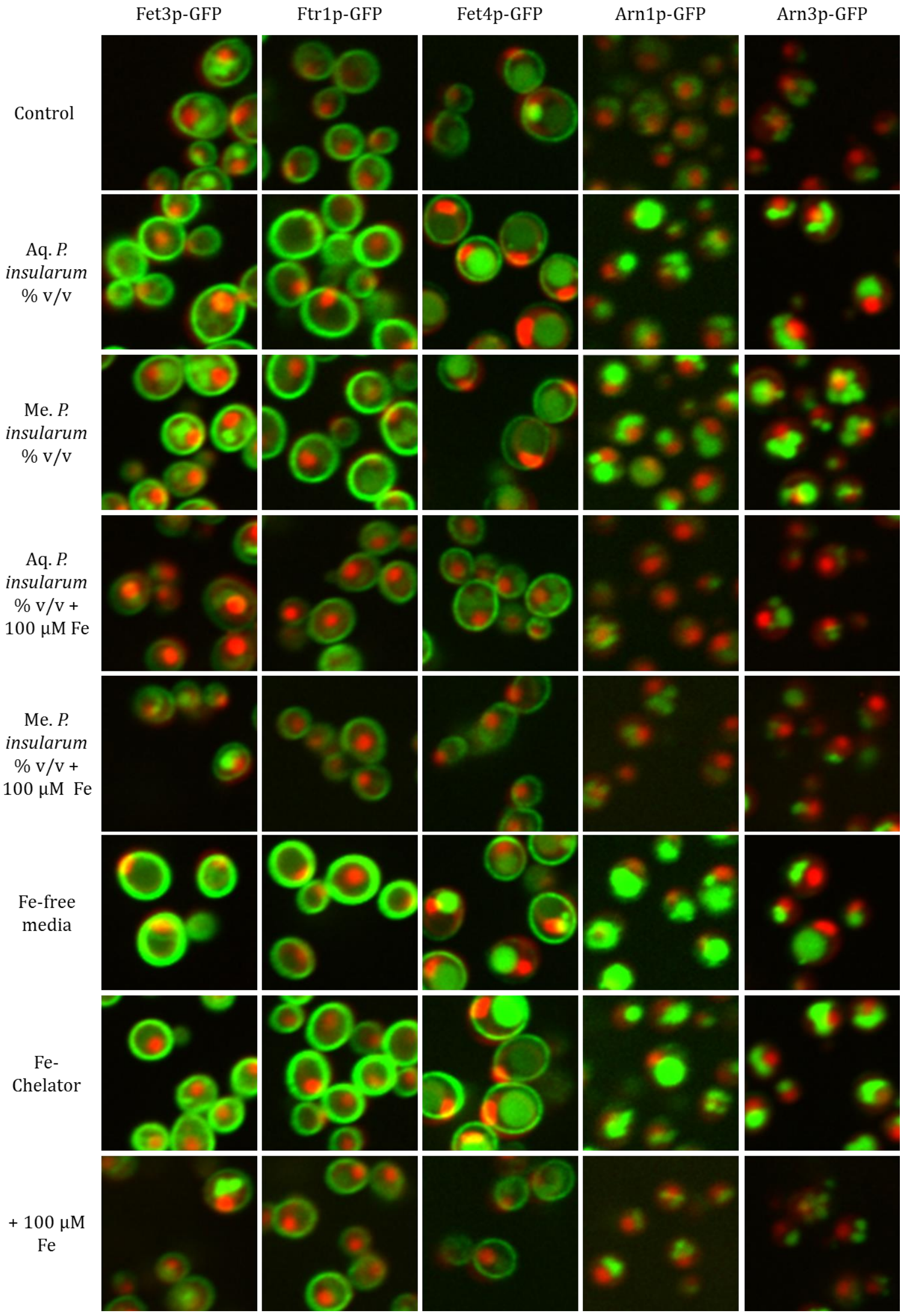


Figure 3.11: Expression levels of iron transporters under extract treatment, monitored via GFP

tagged proteins. Expression levels of iron transporters from the high affinity transport system (Fet3p and Ftr1p), low affinity iron transport system (Fet4p), and iron siderophore transport system (Arn1p and Arn3p) were monitored through their GFP-tagged counterparts, and grown under 8 treatment conditions, including control, aqueous $P$. insularum extract $(0.05 \% \mathrm{v} / \mathrm{v})$, methanol $P$. insularum extract $(0.005 \% \mathrm{v} / \mathrm{v})$, aqueous extract treatment supplemented with $100 \mu \mathrm{M} \mathrm{FeCl}_{3}$, methanol extract treatment supplemented with $100 \mu \mathrm{M} \mathrm{FeCl}_{3}$, cells grown in iron-free media, iron chelator control $(0.1 \mu \mathrm{M})$, and a $100 \mu \mathrm{M} \mathrm{FeCl}_{3}$ supplementation. The GFP-tagged strains were grown overnight, then inoculated into the relevant treatment media at $5 \times 10^{5}$ cells $/ \mathrm{mL}$, and incubated at $30^{\circ} \mathrm{C}$ whilst shaking overnight for $15 \mathrm{~h}$. Cell were diluted into fresh treatment media and $50 \mu \mathrm{L}$ were visualized using the Opera EvoTec spinning disc confocal microscope, where GFP and mCherry/RedStar2 were excitation was achieved at 488 and 561 $\mathrm{nm}$, and detection was through 520/35 and 600/40 filters respectively.
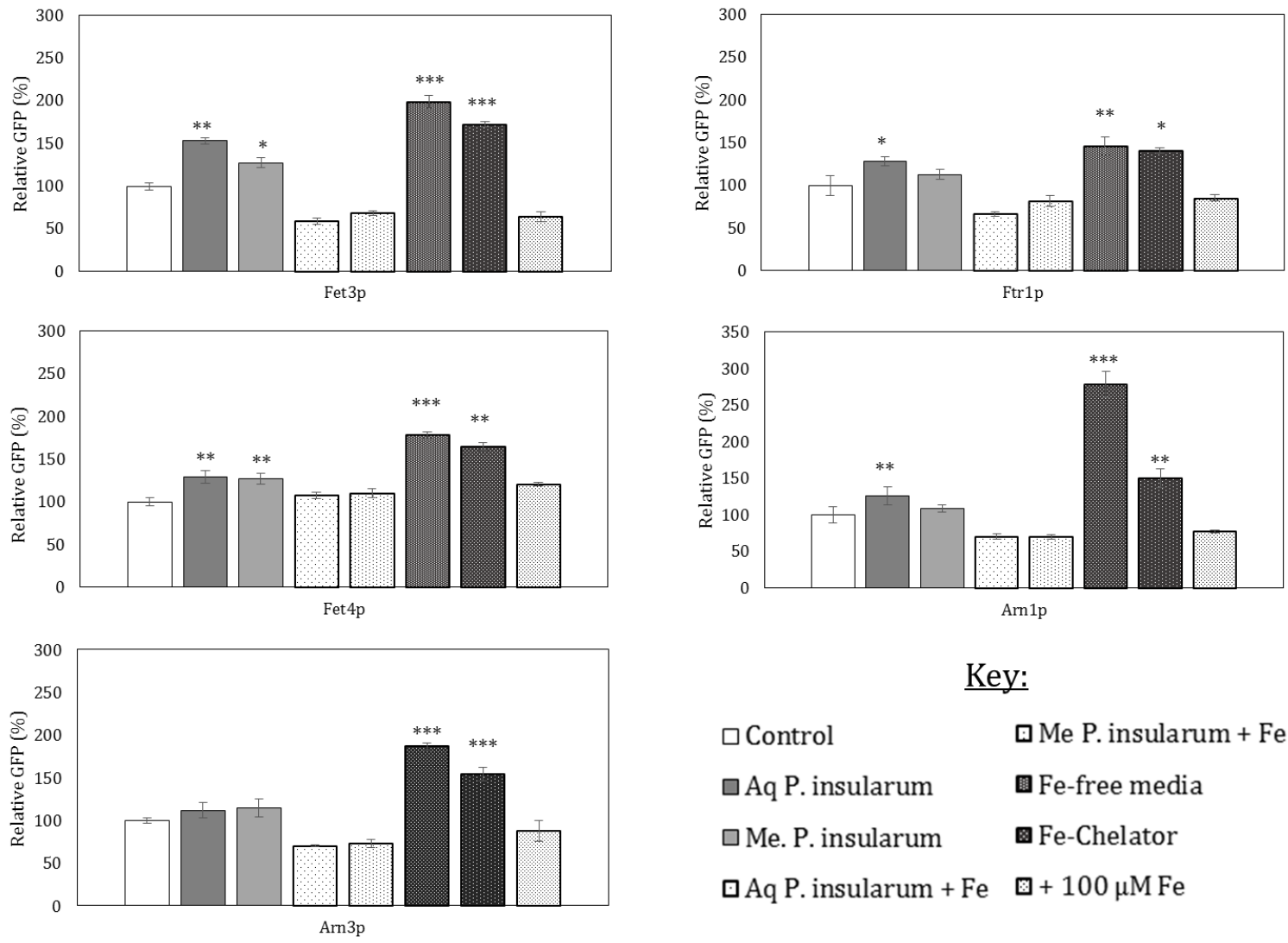

Key:

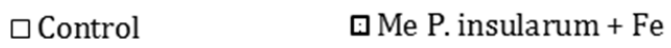

$\square$ Aq P. insularum

- Fe-free media

$\square$ Me. P. insularum

圆e-Chelator

$\square$ Aq P. insularum $+\mathrm{Fe} \quad$ o $+100 \mu \mathrm{MFe}$ 
Figure 3.12: Quantification of GFP intensity of iron transporters upon $P$. insularum treatment. Images obtained from confocal microscopy of iron transporters tagged with GFP were exposed to Acapella software to quantify GFP intensity, utilizing the mCherry and RedStar2 nuclear localising and the cytoplasmic signals respectively. Identification of the cellular periphery allowed for the quantification of the GFP signal, and the median GFP intensity was determined. Average of 3 replicates per treatment was calculated, and compared to control GFP intensity to determine relative GFP (\%) as illustrated. The 8 treatments tested were control, aqueous extract $(0.05 \% \mathrm{v} / \mathrm{v})$, methanol extract $(0.005 \% \mathrm{v} / \mathrm{v})$, aqueous extract treatment supplemented with $100 \mu \mathrm{M} \mathrm{FeCl}_{3}$, methanol extract treatment supplemented with 100 $\mu \mathrm{M} \mathrm{FeCl} 3$, iron free media, iron chelator control $(0.1 \mu \mathrm{M})$, and $100 \mu \mathrm{M} \mathrm{FeCl}{ }_{3}$ supplementation control. Statistical analyses using a one-tailed Student's t-test was carried out to identify if the increase in GFP signal from treatment was statistically significant compared to control $\left(^{*}=\right.$ p-value $<0.05,{ }^{* *}=$ p-value $<$ $0.01, * * *=$ p-value $<0.001)$.

\subsubsection{P. insularum extracts induce a respiratory deficient phenotype}

The growth pattern of yeast strains under specific media conditions often provides information regarding the effect of a growth condition on the strain; for instance, an extended lag phase and a lower stationary phase often indicates a defect in the respiratory system of yeast (Xu et al., 2012). Given that $P$. insularum extracts reduce intracellular iron in the cell (Fig 3.10), and the requirement of iron in heme and iron sulfur cluster (ISC) cofactors needed for respiration (Philpott, 2012), we hypothesized that the extracts of $P$. insularum would induce a growth profile indicative of respiratory deficiency. To test this hypothesis, BY4741 WT yeast was grown in the presence and absence of the aqueous $(0.05 \% \mathrm{v} / \mathrm{v})$ and methanolic $(0.005 \% \mathrm{v} / \mathrm{v})$ extracts of $P$. insularum. The growth pattern of BY4741 grown in the presence of $P$. insularum extracts compared to BY4741 grown under control conditions followed the predicted pattern, whereby extract treated cells exhibited a modestly extended lag phase, with a discernible reduced stationary phase. These findings support the hypothesis that the extracts of $P$. insularum induce a respiratory deficient phenotype. 

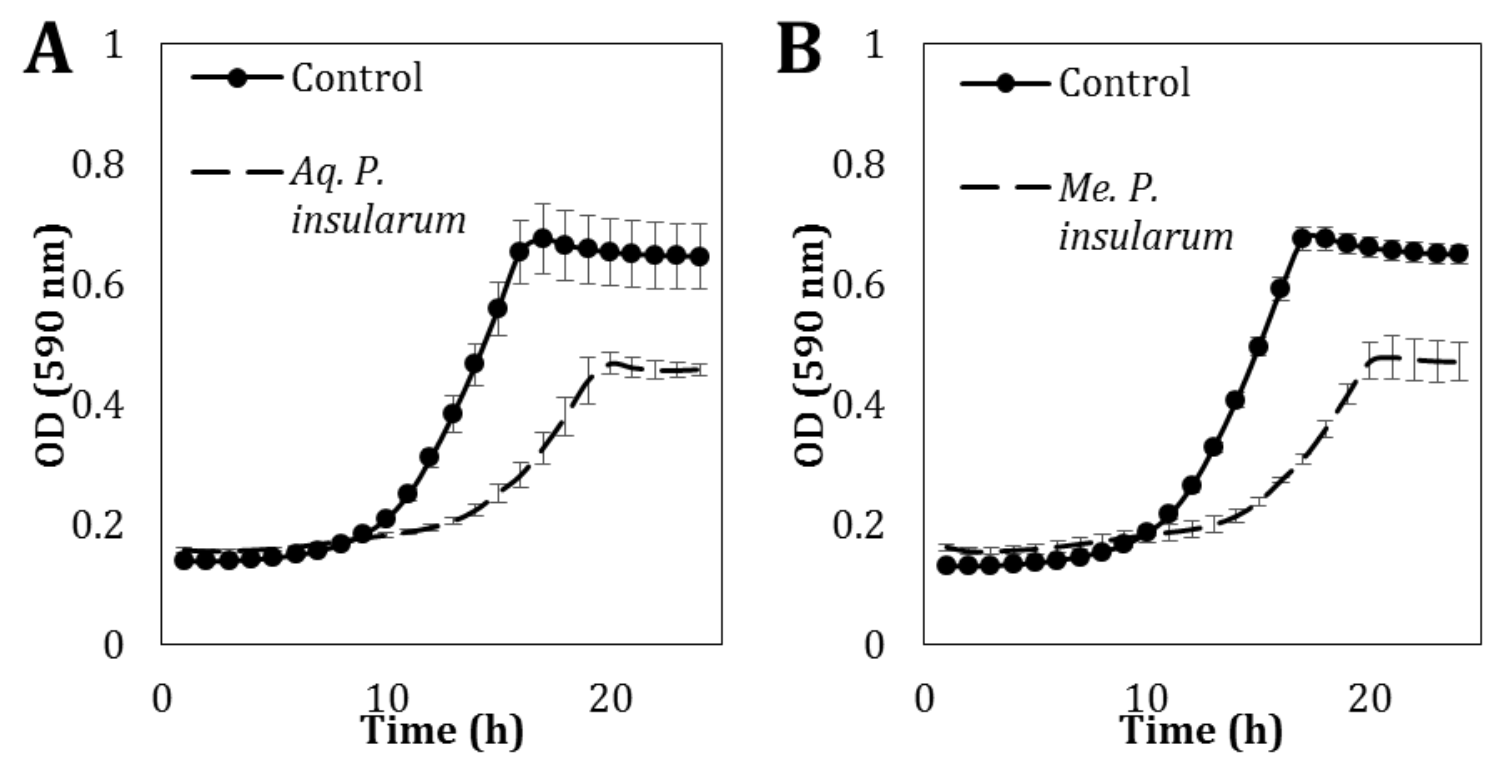

Figure 3.13: Respiratory deficient phenotype of BY4741. Cells grown to log phase were inoculated into the relevant media at $5 \times 10^{5} \mathrm{cells} / \mathrm{mL}$ and grown at $100 \mu \mathrm{L}$ in 96 -well plates, with absorbance readings every hour for $48 \mathrm{~h}$ at $30^{\circ} \mathrm{C}$. A: Growth curve of BY4741 WT under control conditions compared to growth in the presence of the aqueous extract of $P$. insularum at $0.05 \% \mathrm{v} / \mathrm{v}$ (dashed line). B: Growth curve of BY4741 WT under control conditions (solid black line) and in the presence of the methanol extract of $P$. insularum at $0.005 \% \mathrm{v} / \mathrm{v}$ (dashed line).

\subsubsection{P. insularum extracts reduce heme synthesis}

The quantified low levels of iron in the cell (Fig 3.10), in conjunction with the respiratory deficient phenotype (Fig 3.13) in the presence of the extracts of $P$. insularum suggested that the treatment of cells with the extracts led to reduced synthesis of iron-requiring co-factors and a downregulation of iron-requiring pathways. Given that iron is utilized in the cell as an essential transition metal in the activity of heme-dependent and iron-sulfur cluster (ISC) -dependent enzymes and protein complexes (Philpott, 2012) in cellular respiration, we quantified heme in cells that were grown in the presence and absence of $P$. insularum extracts. This was achieved by using the triton methanol method (Padney et al., 1999) on cell lysates of cells harvested from several treatments, including control, aqueous extract treatment, methanolic extract treatment, iron-free media and iron chelator treatment. The quantified heme content of BY4741 WT cells showed marked differences between the control and the four treatments (Fig 3.14). Cells treated with aqueous and 
methanolic $P$. insularum extract showed approximately $50 \%$ reduction in heme content compared to control cells. Likewise, cells grown in iron-free media or in the presence of the iron chelator showed approximately $30 \%$ reduction in heme content compared to control cells. These findings suggest that the reduced levels of heme detected from the four treatments were a consequence of low bioavailable iron, although this is not reflection in status of iron-requiring proteins.

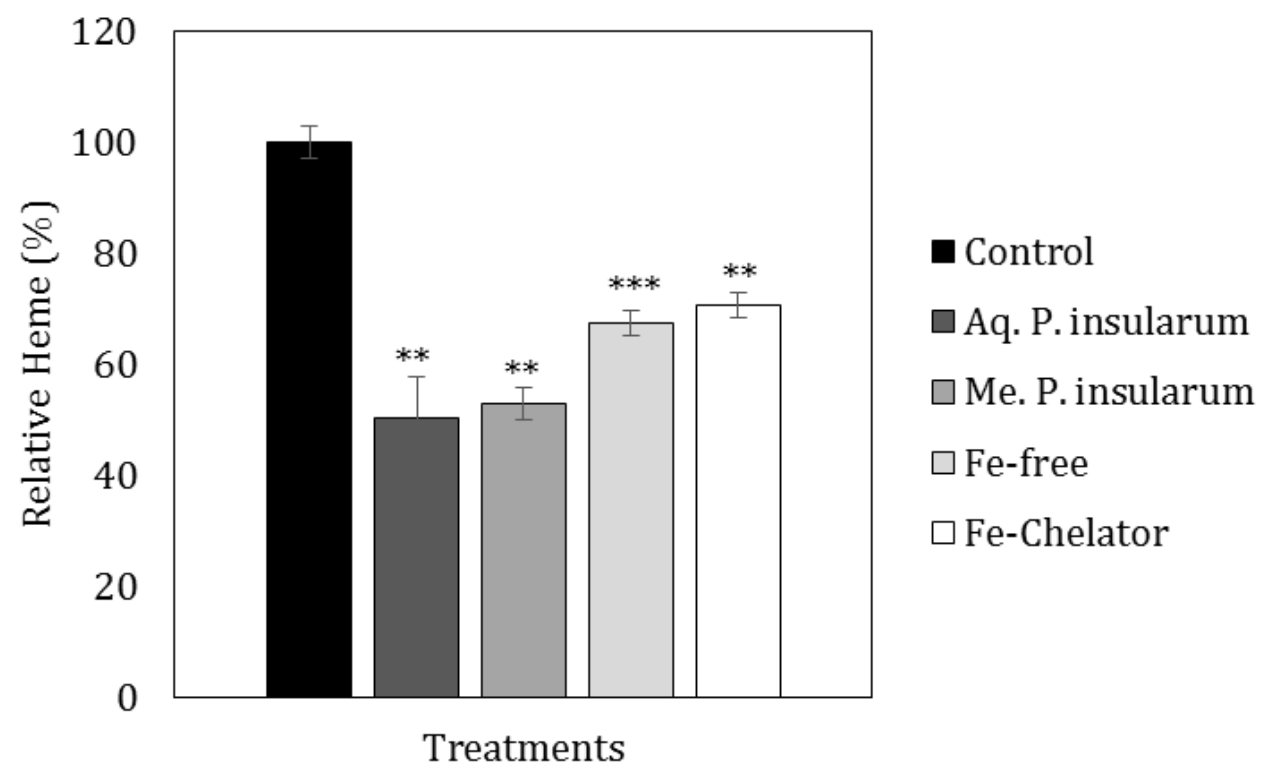

Figure 3.14: $\quad$ Relative heme content of WT treated with P. insularum extracts. BY4741 WT cells were grown in five (5) conditions, including control, aqueous $P$. insularum extract treatment, methanol $P$. insularum extract treatment, iron free media and BPS chelator treatment. Cells were harvested and protein prepared. The protein content was assayed for heme content using the triton methanol method. The standard curve was used to calculate total heme, and the protein content per sample was used to normalize heme content. Relative heme was calculated using the control heme content. Statistical analyses was carried out using Microsoft Excel paired two sample for means, one tailed t-test $(*=p$-value $<0.05,{ }^{* *}=$ p-value $<0.01,{ }^{* * *}=$ p-value $<0.001$ ) .

\subsubsection{P. insularum extracts chelate iron}

Our findings thus far have shown comparable results between the $P$. insularum extracts and the iron chelator BPS. For instance, both $P$. insularum extracts and iron chelator decreased total intracellular iron content (Fig 3.10). Further, treatment of cells with extracts as well as iron chelator caused an up-regulation of iron transporters (Fig 3.11 \& 3.12), indicative of the induction of the low iron response in yeast. Moreover, we have shown that treatment with $P$. insularum extracts and iron 
chelator led to reduced synthesis of heme (Fig 3.14) that was indicative of low bioavailable iron yeast. Therefore, we hypothesized that the bioactive component of the aqueous and methanolic extracts of $P$. insularum mediate its therapeutic effect via an iron chelation mechanism. To test this hypothesis, we employed the chrome azurol S (CAS) assay, a colorimetric assay that indicates whether a compound chelates iron (Alexander \& Zuberer, 1991). The assay was carried out over a concentration range of the $P$. insularum aqueous extract $(0.005 \%-10 \% \mathrm{v} / \mathrm{v})$ and the $P$. insularum methanolic extract $(0.0005 \%-1 \% \mathrm{v} / \mathrm{v})$, as well as EDTA as an established iron chelator to give the required blue-to-yellow colour reaction. The BPS iron chelator used in previous experiments was not used since it produced a different colour reaction as it forms a red complex with iron. The CAS assay results against the aqueous and methanolic extracts of $P$. insularum show moderate blue-to-yellow colour change as soon as the extracts were added to the CAS solution (Fig $3.15 \mathrm{~A}$ ), and this colour change intensified after $1 \mathrm{~h}$ incubation (Fig 3.15B). However, unlike EDTA that showed this positive blue-to-yellow colour change down to $0.0001 \mu \mathrm{M}$ EDTA, the aqueous extract of $P$. insularum was only able to induce a colour change down to $0.5 \% \mathrm{v} / \mathrm{v}$, while the methanol extract of $P$. insularum was able to induce a colour change at concentrations as low as $0.1 \% \mathrm{v} / \mathrm{v}$. These findings show that although the extracts of $P$. insularum chelate iron, their iron chelation activity was not as potent as that of EDTA. However, these findings are not directly comparable as the $P$. insularum extracts tested are crude extracts and may contain miniscule amounts of the bioactive component.

\begin{tabular}{|c|c|c|c|c|c|c|c|c|c|}
\hline A & 1 & 0.5 & 0.1 & 0.05 & \multirow{2}{*}{$\begin{array}{l}\text { B } \\
\text { Aq. P. insularum } \\
(\% \mathrm{v} / \mathrm{v})\end{array}$} & 1 & 0.5 & 0.1 & 0.05 \\
\hline $\begin{array}{r}\text { Aq. P. insularum } \\
(\% \mathrm{v} / \mathrm{v})\end{array}$ & & & & & & & & & \\
\hline & 0.1 & 0.05 & 0.01 & 0.005 & & 0.1 & 0.05 & 0.01 & 0.005 \\
\hline $\begin{array}{r}\text { Me. P. insularum } \\
(\% \mathrm{v} / \mathrm{v})\end{array}$ & 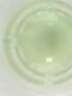 & & & & $\begin{array}{r}\text { Me.P. insularum } \\
(\% \mathrm{v} / \mathrm{v})\end{array}$ & & & & \\
\hline & 0.05 & 0.01 & 0.005 & 0.001 & & 0.05 & 0.01 & 0.005 & 0.001 \\
\hline $\begin{array}{r}\text { EDTA } \\
(\mu \mathrm{M})\end{array}$ & & & & & $\begin{array}{r}\text { EDTA } \\
(\mu \mathrm{M})\end{array}$ & & & & \\
\hline
\end{tabular}


Figure 3.15: $\quad$ CAS assay illustrating iron chelation activity of $\boldsymbol{P}$. insularum extracts. $100 \mu \mathrm{L}$ of CAS assay solution was aliquot in replicate into 96-well plates, to which $100 \mu \mathrm{L}$ of the relevant dilution of extract (\% v/v) or EDTA $(\mu \mathrm{M})$ was added, and images were taken at $0 \mathrm{~min}$ and $1 \mathrm{~h}$ after leaving at RT. A: CAS assay at t0, soon after extracts and EDTA were added to the CAS solution. B: CAS assay at $1 \mathrm{~h}$ after extracts and EDTA were added to the CAS solution. Color change from blue to yellow indicates a positive iron chelation activity.

\subsubsection{P. insularum extracts exhibit an anti-inflammatory response in murine macrophages}

Aside from its extensive use in ailments generally attributed to spirits and ghosts, the aqueous extract from $P$. insularum leaves is also used to treat skin infections, skin wounds and general body aches and swellings in Samoa (Whistler, 1996) indicative of the extracts playing a modulating role in inflammation. To test if $P$. insularum targets inflammation as it is currently prescribed in traditional Samoan medicine, the effects of varying concentrations of the aqueous and methanolic extracts of $P$. insularum on the cellular metabolic rate (MTT), the anti-inflammatory cytokine interleukin-10 (IL-10), the pro-inflammatory cytokine interleukin-12 (IL-12), tumour necrosis factor $\alpha(\mathrm{TNF} \alpha)$ and nitrous oxide (NO) levels were monitored in activated primary macrophages derived from mice (C57BL/6). Primary macrophages were also treated with varying concentrations of the iron chelator BPS to ascertain if the results obtained from each extract treatment were a consequence of its iron chelator activity. Additionally, varying concentrations of Risperidone, an antiinflammatory control (O'Sullivan et al., 2014) was included to determine if the $P$. insularum extracts exhibited the hallmark phenotypes of an anti-inflammatory compound particularly in increasing IL10 and decreasing IL-12 levels.

All four concentrations of the aqueous $P$. insularum extract $(0.5,1,2$ and $3 \% \mathrm{v} / \mathrm{v})$ and the two higher concentrations of the methanolic extract $(0.05$ and $0.1 \% \mathrm{v} / \mathrm{v})$ increased levels of MTT by 50-75\% compared to untreated macrophages (Fig 3.16A). In contract, the two lower concentrations of the methanolic $P$. insularum extract $(0.005$ and $0.01 \% \mathrm{v} / \mathrm{v})$, the three lower concentrations of iron chelator $(0.01,0.1$ and $1 \mu \mathrm{M})$ and all four Risperidone concentrations $(6.25,12.5,25$ and $50 \mu \mathrm{M})$ did 
not increase MTT levels, suggesting that these treatments did not alter metabolic activity of treated cells compared to the control. However, the highest tested concentration of iron chelator $(10 \mu \mathrm{M})$ showed reduced metabolic activity, which may indicate reduced viability. While it is ideal that MTT levels were not lower compared to control in extract treatments, the increased MTT levels suggest that a component of the crude extracts may function to increase the metabolic activity of macrophages. The increased metabolic activity of macrophages in the presence of the extracts can also be interpreted as a bioactivity marker, aside from cytokine measurements.

Levels of the anti-inflammatory cytokine IL-10 were markedly increased in a dosedependent manner with the four concentrations of the aqueous extract of $P$. insularum (Fig 3.16B), with increases from $50 \%$ at the lowest concentration tested $(0.5 \% \mathrm{v} / \mathrm{v})$ to $800 \%$ at the highest concentration tested $(3 \% \mathrm{v} / \mathrm{v})$. Interestingly, the $P$. insularum methanolic extract did not alter IL-10 levels at any concentration compared to untreated macrophages. The two higher concentrations of the iron chelator BPS increased IL-10 levels by $600-700 \%$ (6-7-fold) compared to control macrophages, a result that is comparable to the $800 \%$ increase in IL-10 levels observed from the highest concentration tested of the aqueous extract of $P$. insularum. The four Risperidone concentrations increased IL-10 levels by $100 \%$ compared to untreated macrophages.

Levels of the pro-inflammatory cytokine IL-12 were markedly reduced with the four treatments of the aqueous or methanolic extracts of $P$. insularum, compared to untreated macrophages (Fig 3.16C). The lowest dose of the aqueous extract reduced IL-12 levels by 40\%, while the three other doses reduced IL-12 levels by $95-100 \%$ compared to untreated macrophages. The four $P$. insularum methanolic extract concentrations significantly reduced IL-12 levels by $25-80 \%$ compared to untreated macrophages. The two higher concentrations of the iron chelator as well as the two higher concentrations of Risperidone reduced IL-12 levels by $60-75 \%$ compared to untreated macrophages.

Levels of TNF $\alpha$ were dramatically increased with the two higher concentrations of the aqueous $P$. insularum extract with increases of $650-4000 \%$ compared to untreated macrophages (Fig 
3.16D). Three of the four $P$. insularum methanolic extract treatments $(0.005,0.01,0.1 \% \mathrm{v} / \mathrm{v})$ increased TNF $\alpha$ levels by $20-110 \%$ compared to untreated macrophages. The iron chelator at all four tested concentrations and Risperidone at the two lower concentrations did not significantly alter TNF $\alpha$ levels compared to untreated macrophages, while the two higher risperidone concentrations reduced TNF $\alpha$ levels by approximately 50\% compared to untreated macrophages. Interestingly, NO levels were increased in cytokines from macrophages treated with the aqueous extract of $P$. insularum, yet were unaltered in macrophages treated with the methanolic extract of $P$. insularum, the iron chelator or risperidone (Fig 3.16E).

The analyses of cytokine analyses from primary macrophages treated with the aqueous and methanolic extracts of $P$. insularum produced varied responses. The aqueous extract increased IL-10 levels and decreased IL-12 levels, hallmark indicators of an anti-inflammatory compound, consistent with the anti-inflammatory application of the plant in Samoan traditional medicine. However, the methanolic extract was only able to reduce IL-12 levels without affecting IL-10 levels. Further, these findings showed that the aqueous extract of $P$. insularum was more potent in regulating IL-10, IL-12, TNF $\alpha$ and NO levels compared to the methanolic extract.

Figure 3.16: Macrophage response to treatment with $P$. insularum extracts. Primary macrophages were isolated from mice and treated with LPS to induce the inflammatory response for $24 \mathrm{~h}$. The macrophages were then treated with the relevant aqueous (Aq. P. insularum) or methanolic (Me. P. insularum) extract concentration as well as the iron chelator control (Fe-Chelator) and the antiinflammatory control (Risperidone) at various concentrations. A: MTT results for the extract and control treatments. B: IL-10 levels from the macrophages reported as relative IL-10 compared to the relevant treatment control. C: IL-12 levels from the macrophages reported as relative IL-12 compared to the relevant treatment control. D: TNFA levels from the macrophages reported as relative TNFA compared to the relevant control. E: NO levels from the treated macrophages reported as relative NO compared to the relevant control. Statistical analyses were carried out using a one-tailed Student's t-test ${ }^{*}$ : p-value $<0.05$; **: p-value $<0.01 ; * *$ : p-value $<0.001)$. 
A
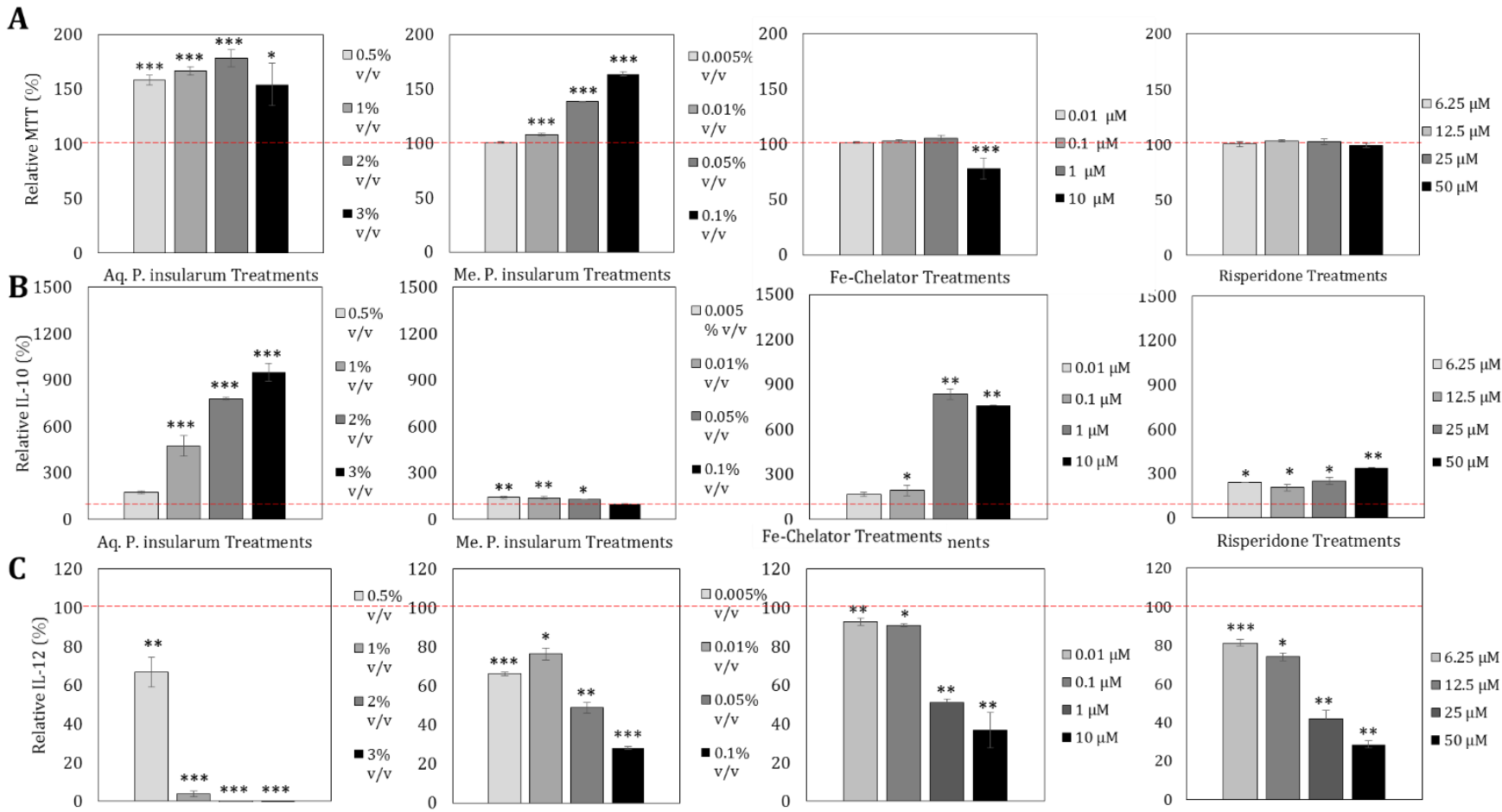

Me.P. insularum Treatments
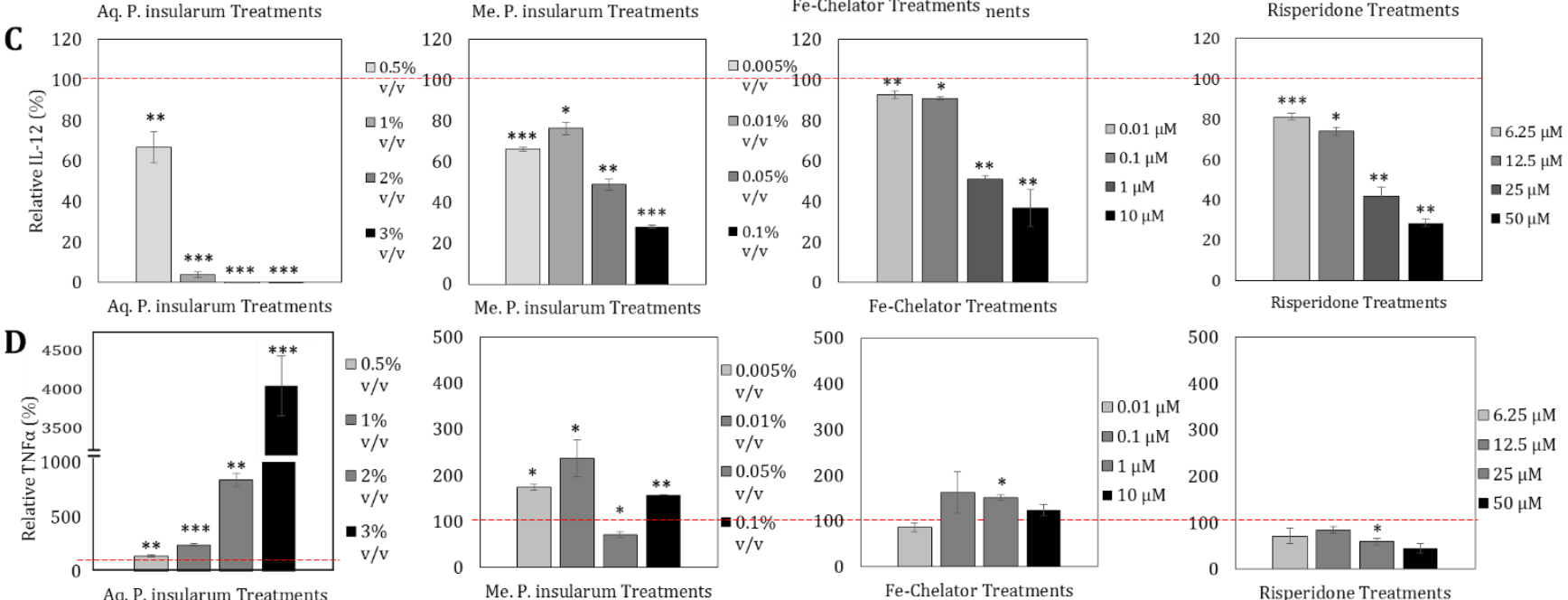

Fe-Chelator Treatments

Risperidone Treatments
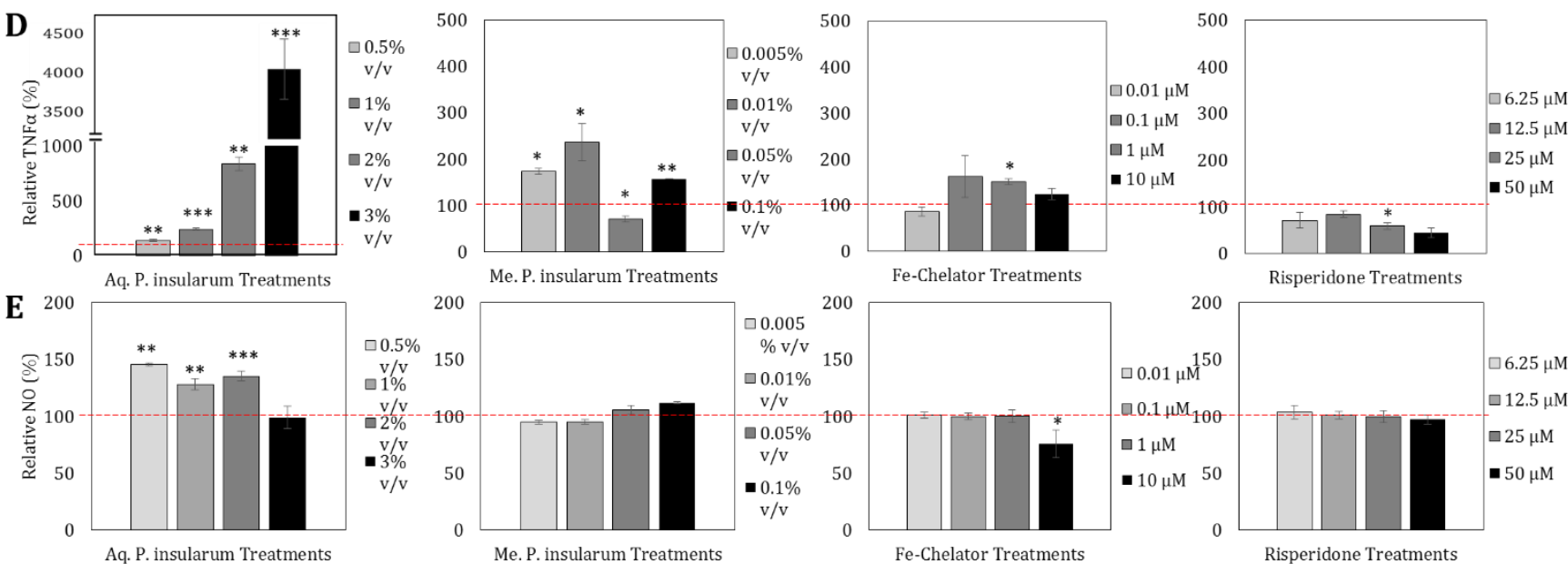

\section{macrophages}

We previously determined from analyses in yeast that the extracts of $P$. insularum affected mitochondria respiration (Fig 3.13) via reduced heme synthesis (Fig 3.14). To investigate if mitochondrial function was affected by $P$. insularum extracts in murine macrophages, the enzymatic activity of aconitase, an iron-sulfur dependent enzyme involved in the TCA cycle, was measured in macrophages treated with the extracts of $P$. insularum, the iron chelator BPS or the anti- 
inflammatory control Risperidone. Compared to untreated control, the relative aconitase activity was significantly decreased with the aqueous $P$. insularum extract, the methanolic $P$. insularum extract, and the iron chelator treatments (Fig 3.17). Importantly, Risperidone-treated macrophages had aconitase activity levels that were significantly increased compared to the control. These results implied that similar to yeast cells, treatment of mammalian cells with extracts of $P$. insularum affected the mitochondrial machinery involved in cellular respiration.

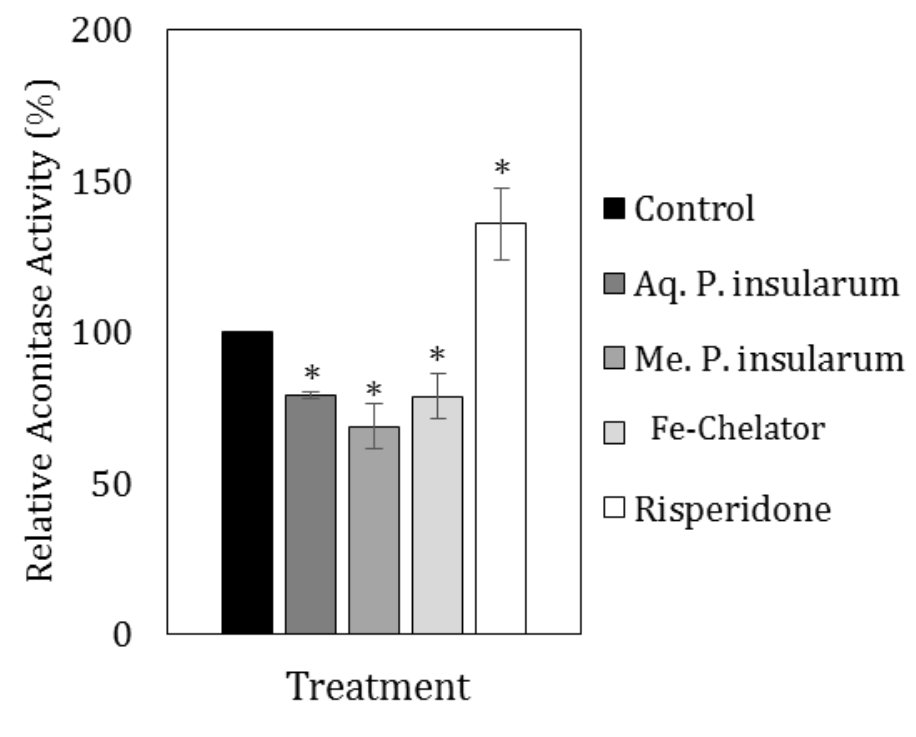

Figure 3.17: The aconitase activity of macrophages. Macrophages were induced with LPS followed by treatment with extracts, BPS or risperidone. Protein was isolated from the macrophages, which were used for the aconitase assay. Activity of aconitase from protein samples were calculated, followed by comparisons between each treatment and its relevant vehicle control. Presented is the relative aconitase activity per treatment normalized to its vehicle control. Statistical analyses were carried out using a onetailed Student's t-test $(*$ : p-value $<0.05)$. 


\subsection{Discussion}

This chapter aimed to elucidate the MOA of the leaf extracts from the plant $P$. insularum, administered in traditional Samoan medicine to treat illnesses accredited to supernatural causes as well as inflammation related ailments. Results that have been presented herein have indicated that the extracts of $P$. insularum, despite different preparations as aqueous and methanolic extractions, exhibited an iron chelator MOA. It is postulated that despite the different extractions, the bioactive components of the crude extracts may belong to the same group of compounds with the same underlying iron chelating mechanism, as has been observed from other natural products isolated from the same source, such as the microtubule stabilizing mechanism of Peloruside A (extracted with aqueous methanol) and B (extracted with methanol) from Mycale hentscheli (Brakovic \& Harvey, 2015; Singh et al., 2010; West et al., 2000).

In yeast cells, the extracts of $P$. insularum were shown to induce a hypersensitive growth phenotype in strains lacking iron transport genes (Figs 3.3, 3.4 \& 3.5), which indicated that iron transporters were required to buffer the effects of the extracts. This suggested that either iron transport into the cell was blocked, or that iron was not available for cellular processes. However, because all iron transporters were shown to exhibit the hypersensitive phenotype (Figs 3.4 \& 3.5), it was unlikely that an iron transporter was a physical target of the extracts because deletion of the extract target would render the deletion mutant resistant to the extract (Smith et al., 2010). We therefore proposed the extracts exhibited their activity by making iron unavailable to the cell via the chelation of iron. This hypothesis was supported when the addition of exogenous iron rescued the extract-induced growth defect (Fig 3.6), the interpretation being that iron pools which have been depleted by the extract would have been replenished to some extent depending on the concentration of the extract treatment. Our iron chelator hypothesis was further corroborated when we determined that extracts reduce intracellular iron compared to control cells to the same extent that the established iron chelator BPS reduced intracellular iron by (Fig 3.10). This phenotype was also observed in yeast cells treated with curcumin, which was also proposed to possess iron 
chelating activity (Minear et al., 2011).Interestingly, data from the ICP-MS quantification of iron did not allow for the determination of whether iron was chelated inside the cell or outside the cell.

Theoretically, the chelation of iron intracellularly would suggest the complex remained inside the cell, and complexed iron would be released upon treatment for quantification, leading to increased intracellular total iron (Tamarit et al., 2006). However, despite the internalization of curcumin by the cell, its iron content remained reduced (Minear et al., 2011), suggesting that the curcumin-iron complex is removed before iron was quantified by ICP-MS possibly when insoluble cell debris is removed. Further, the iron chelator control used in this study chelates iron extra-cellularly, which may suggest that the reduced iron content determined from the extract treatment, similar to that obtained for the chelator control, was a consequence of extracellular iron chelation. However, because both internalized curcumin iron chelation and extracellular BPS iron chelation produce reduced iron content in treated cells, our results were thus unable to provide insight into the site of iron chelation by the $P$. insularum extracts.

Yeast cells undergo a molecular response under low iron conditions that includes the down-regulation of iron-requiring pathways, and the up-regulation of iron transporter expression (Chen et al., 2004; Kaplan et al., 2006; Philpott et al., 2012; Philpott \& Protchenko, 2008; Puig et al., 2005; Shakoury-Elizeh et al., 2004; Shakoury-Elizeh et al., 2012). When yeast strains with GFP-tagged iron transporters were treated with $P$. insularum extracts, it was determined that the expression levels of the iron transporters were noticeably increased similar to cells treated with the iron chelator control as well as cells grown in iron-free media (Fig 3.11). Our results are consistent with previous work that monitored the expression of the iron transporter Fet3p using a lacZ reporter system and found that Fet3p expression was upregulated under low iron conditions (Chen et al., 2004; Puig et al., 2005).

As proposed by Xu et al. (2012), an extended lag-phase and lower stationary phase were indicative of a defect in cellular respiration. This phenotype was observed from the growth curves of yeast treated with $P$. insularum extracts, indicating that the extracts caused a defect in cellular 
respiration. The requirement of iron in cellular respiration in the form of iron sulfur cluster proteins and heme is well understood (reviewed in Philpott et al., 2012). Thus, heme was quantified and our findings showed that heme levels were significantly reduced in extract treated cells compared to control cells, a result that is consistent with the downregulation in heme biosynthesis (Puiget al., 2005; Shakoury-Elizeh et al., 2010). The reduced heme(Fig 3.14), and presumably reduced activity of iron sulfur cluster dependent enzymes (Puig et al., 2005) contribute to the reduced stationary phase of extract-treated cells, as it is during this phase that yeast cells are shunted to cellular respiration as the more readily fermented glucose carbon source is depleted (Shakoury-Elizeh et al., 2010).

Results from the CAS assay definitively showed that the $P$. insularum extracts chelate iron (Fig 3.17). The CAS solution turned from blue (with complexed iron) to yellow (iron is removed by a chelator) upon the addition of both the aqueous and methanolic extracts of $P$. insularum. Interestingly, the CAS assay carried out here showed a similar chelating strength between the aqueous and methanolic extracts, although it appeared that the aqueous extract chelated iron more readily than the methanolic extract, as the aqueous extract caused a colour change more readily than the methanolic extract. These findings may suggest that the aqueous extract iron chelating component more readily chelates iron, while its variant in the methanolic extract, present at higher concentrations may not readily chelate iron, and may explain its higher potency yet slower iron chelating activity as observed. It is important to note here that given the well documented metalchelating activity of plant natural products such as plant polyphenols and flavonoids (Hatcher et al., 2008; Hider et al., 2001; Morel et al., 1993), it would be unsurprising to obtain a positive colour change if we were to include another plant extract in the CAS assay.

In addition to iron transport gene deletions, the deletions of TEF4, SNF5, BEM1 and BEM4 that were determined to be hypersensitive to the aqueous extract in the genome-wide analysis (Fig 3.2) may be secondarily affected by the low iron condition resulting from the $P$. insularum extracts. The tef4 strain exhibits fragmented vacuoles (Esposito \& Kinzy, 2010) and deletion mutants with fragmented vacuoles are hypersensitive to low iron conditions (Szcypka et al., 1997). SNF4 is a 
component of the SWI/SNF (SWItch/Sucrose Non-Fermentable) complex, which is important for transcriptional regulation of iron uptake at the chromatin level (Monahan et al., 2008). BEM1 and BEM4 are involved in cell budding and therefore the cell cycle, a process that can be arrested by iron chelators (Fu et al., 2007; Rao, 2013; Siriwardana \& Seligman, 2013; Zhang, 2014).

Collectively, we use our results in yeast to propose a model wherein the bioactive compound from the aqueous and methanolic extracts of $P$. insularum chelates iron either intracellularly or extracellularly, leading to reduced bioavailable iron for cellular processes required for growth, such as cellular respiration (Fig 3.18). The cell subsequently responds by upregulating the expression of iron transporters to import more iron into the cell and downregulate iron-requiring cellular processes. This is a working model that requires further investigation in mammalian cells, such as the measurements of intracellular iron in murine macrophages. As heme is required for ergosterol synthesis (Lewis et al., 1985), it would be interesting to incorporate ergosterol synthesis into this model.
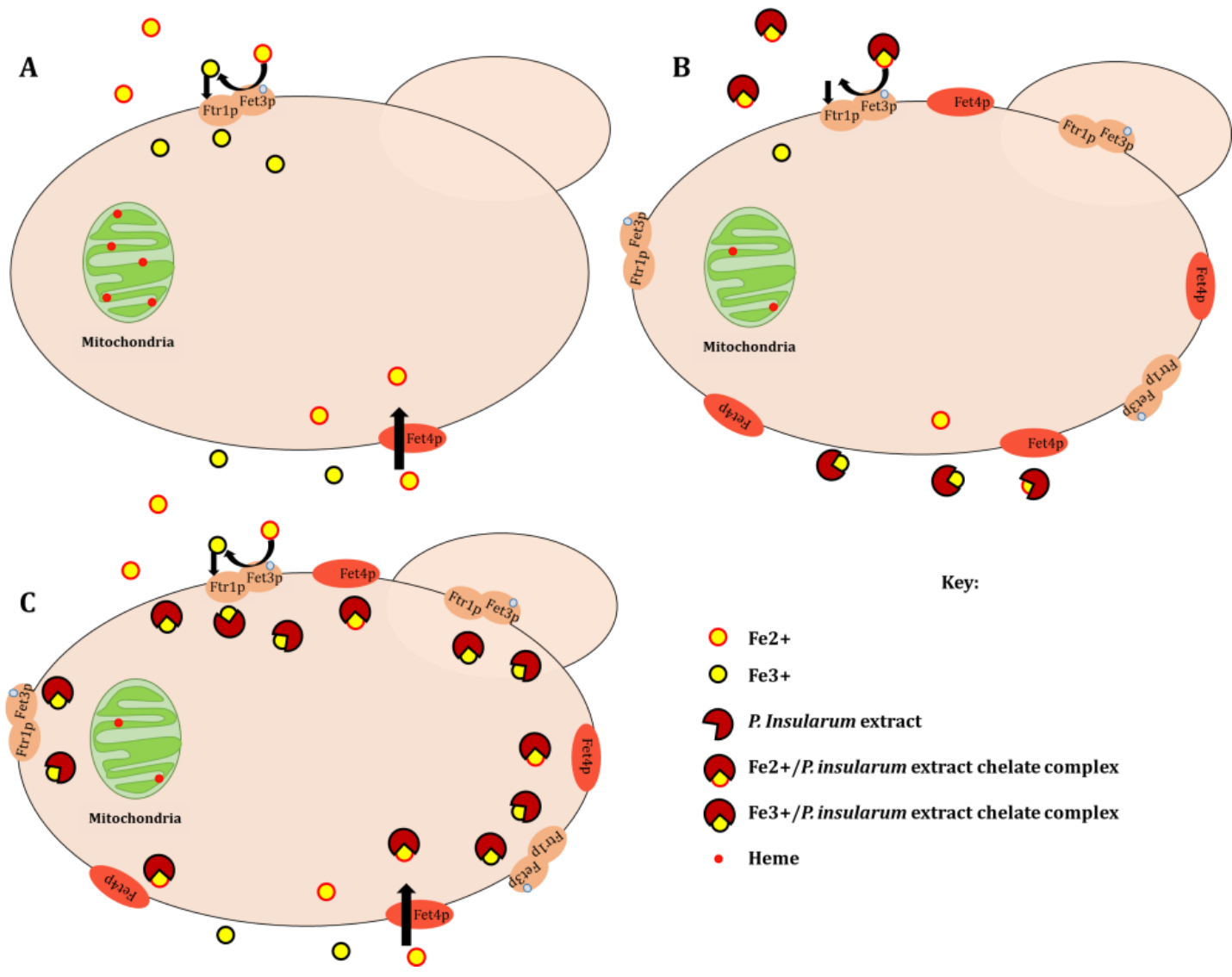
Figure 3.18: Model of the mechanism of action of $\boldsymbol{P}$. insularum extracts. A: Iron replete conditions. The cell has sufficient iron for iron requiring proteins, enzymes and co-factors. B: Extracellular iron chelation. $P$. insularum extracts chelate iron outside of the cell, and the cell subsequently is unable to internalize more iron, despite up-regulation of iron transporter expression; reduced heme production results from low iron. C: Intracellular iron chelation. P. insularum extracts chelate iron inside of the cell, and the cell subsequently is unable to access the iron complexed in the chelate complex, leading to reduced heme production. Despite up-regulation of iron transporter expression, iron that is internalized is chelated.

Investigations into the effects of the $P$. insularum extracts on inflammation in murine macrophages clearly showed a strong anti-inflammatory activity for the aqueous extract of $P$. insularum and a mild anti-inflammatory activity for the methanolic extract (Fig 3.16). Interestingly, the responses from the iron chelator control in the macrophages closely mirrored the trends observed from the extracts, suggesting the anti-inflammatory activity observed from the extracts was a consequence of iron chelation. These results are consistent with the intimate connection between iron and inflammation (Ganz \& Nemeth, 2015; Wessling-Resnick, 2010). It has been generally reported that high iron promotes infection and subsequently inflammation (Jurado, 1997).Indeed, various iron chelators have been reported to possess anti-inflammatory activities, such as ibuprofen, and curcumin (Islam et al., 2016; Kennedy et al. 1990; Menon \& Sudheer, 2007).

The mechanisms by which iron chelators mediate inflammation are not completely understood. The anti-inflammatory effects of iron chelators were largely attributed to their reactive oxygen species (ROS) scavenging ability, as free iron has the ability to generate ROS and other oxidative stressors (Lehmann et al., 2015). However, evidence has revealed that iron plays a role in the activation of the NF-KB, which drives the inflammatory response (Xiong et al., 2003). Logically then, the removal of iron via iron chelation leads to reduced activation of NF-kB, resulting in an antiinflammatory phenotype.

Intriguingly, the results obtained from the positive anti-inflammatory control Risperidone (O'Sullivan et al., 2014), although similar for IL-10 and IL-12 trends, proved variant in the case of the TNF $\alpha$ results. Reduced TNF $\alpha$ levels are expected under anti-inflammatory conditions, so it is 
paradoxical that despite the anti-inflammatory activity of the extracts of $P$. insularum, the extracts induced increased TNFa levels, indicative of an inflammatory response arising from its pivotal role in the inflammatory response (Esposito \& Cuzzocrea, 2009). However, Zakharova \& Ziegler (2005) showed that injection of mice with TNF $\alpha$ led to the down-regulation of the pro-inflammatory cytokine IL-12, suggesting that TNF $\alpha$ may also possess anti-inflammatory effects, as observed from the macrophage response in $P$. insularum treatment.

In summary, this chapter has proposed a mechanism by which the aqueous extract of $P$. insularum is used in traditional medicine in Samoa. Using yeast as a model organism, we identified that the deletion of iron transporters in the presence of $P$. insularum extracts markedly reduced growth, which was rescued upon supplementation with exogenous iron. Reduced intracellular iron, upregulated iron transporter expression, and reduced heme synthesis together with a respiratory deficient phenotype were the effects the extracts had on yeast cells. These findings were collated to present a model by which the extracts chelate iron away from iron-requiring cellular processes. Additionally, this iron chelation has been further shown to mediate an anti-inflammatory activity in murine macrophages, which can attest to how the plant is used to treat inflammation-related ailments in traditional medicine. 


\section{Chapter 4:}

\section{Haploinsufficiency and Homozygous Profile Analyses of the Aqueous and Methanolic Extracts of Psychotria insularum}

\subsection{Introduction}

Thus far, we have identified the extracts of Psychotria insularum as the most potent extracts from the 11 Samoan medicinal plants collected for this work. We used an agar-based analysis of $\sim 4,300$ non-essential genes to identify iron transport and iron chelation as the mechanisms by which the extracts of $P$. insularum mediated its anti-inflammatory activity. Despite these findings, the agar-based genome-wide analysis of the non-essential genes comprised only $\sim 70 \%$ of the yeast genome against only the aqueous extract of $P$. Insularum, leaving the remaining non-essential genes to be screened against the aqueous extract, and both essential and nonessential genes to be investigated against the methanolic extract of $P$. Insularum. In this chapter, we aimed to utilize the diploid yeast libraries, both in heterozygous and homozygous forms to obtain complete chemical-genetic profiles of both the aqueous and methanolic extracts, via the happloinsufficiency profiling (HIP) and homozygous profiling (HOP techniques.

Complete genome-wide analyses conducted in yeast via the HIP technique utilizes heterozygous diploid mutants of essential and non-essential genes. This approach identifies the most hypersensitive mutant as the genetic target of the compound tested (Giaever et al., 1999). For instance, Giaever et al. (1999) used this approach in a proof-of-concept work using tunicamycin and benomyl, where the correct targets of these two drugs ( $A L G 7$ and TUB1, respectively) were identified. Further, multiple other compounds have been tested using this approach, as well as several other growth conditions such as media, pH and temperature (Giaever et al., 2004; Hillenmeyer et al., 2008; Pierce et al., 2007; Yan et al., 2008). 
Complementary to HIP analysis is the utilization of HOP technique, utilizing diploid deletion mutants of non-essential genes. This approach, given its nature, inherently does not allow for the incorporation of essential genes and therefore only determines the fitness of the deletion mutants against a compound. Instead of identifying genetic targets, the HOP technique identifies gene deletions that are hypersensitive in the presence of a compound, thereby indicating that the gene products are required to buffer the effects of the compound. This in turn provides an outlook to the compound's mechanism of action, based on the buffering pathways identified from the hypersensitive deletion mutants. This approach has been used for instance, by Parsons et al. (2006) to identify calcium disruption as the mechanism of action of the breast cancer drug tamoxifen. The buffering mechanisms of yeast against many other compounds have been determined using this approach (Giaever et al., 2004; Hillenmeyer et al., 2008; Pierce et al., 2007; Yan et al., 2008).

In this chapter, we aim to use the competitive pooled HIP and HOP analyses of the aqueous and methanolic extracts of $P$. insularum to identify genetic targets of both, and any further buffering mechanisms that were not previously identified in the agar-based genome-wide analysis of haploid deletion mutants. The HIP analyses did not identify genetic targets for either the aqueous or methanolic extracts of $P$. insularum, while the HOP analyses identified buffering genes that complemented the iron chelating mechanism of action identified in Chapter 3. 


\subsection{Methods}

\subsubsection{Pooling the HET and HOM libraries}

The heterozygous (HET) and homozygous (HOM) diploid libraries (Open Biosystems) were pinned on YPD+G418 $(100 \mu \mathrm{g} / \mathrm{mL})$ plates in 384 colony format on rectangular petri plates (Singer). After $24 \mathrm{~h}$ incubation at $30^{\circ} \mathrm{C}$, the colonies were pooled twice with $5 \mathrm{~mL}$ of YPD+G418 by agitation with a glass rod across the agar surface and collected in a sterile conical flask on ice. Glycerol was added to a final concentration of $15 \% \mathrm{v} / \mathrm{v}$ before cell density was calculated using a Coulter counter. Aliquots of each library were prepared at $1 \times 10^{9}$ cells and stored at $-80^{\circ} \mathrm{C}$.

\subsubsection{Competitive growth of HET and HOM libraries}

A single aliquot of the pools of HET and HOM libraries was used to inoculate $10 \mathrm{~mL} \mathrm{SC}$ and each culture was grown whilst shaking at $30^{\circ} \mathrm{C}$. After $18 \mathrm{~h}$, this $10 \mathrm{~mL}$ overnight culture was used to inoculate fresh $100 \mathrm{~mL} \mathrm{SC}$ that was incubated at $30^{\circ} \mathrm{C}$ whilst shaking for a further $5 \mathrm{~h}$. This was to ensure cells were in log phase of growth at the initiation of the treatment. Fresh $2 \mathrm{~mL} \mathrm{SC}$ with the relevant concentration of extract (optimized at $0.005 \% \mathrm{v} / \mathrm{v}$ for aqueous extract, and $0.001 \% \mathrm{v} / \mathrm{v}$ for methanolic extract, optimized in Appendix $\mathrm{VI}$ ); was then inoculated to a final concentration of $5 \times 10^{6}$ cells $/ \mathrm{mL}$ (i.e., approximately 1000 representations of each strain) and incubated at $30^{\circ} \mathrm{C}$ whilst shaking for $15 \mathrm{~h}$. This became the first 10 generations. The $\mathrm{OD}_{600}$ was measured to ensure cells were at log phase $\left(\mathrm{OD}_{600}=4-6\right)$ and to ensure the concentration of the extract was sub-lethal $(10-20 \%$ growth inhibition), and cell density was calculated using a Coulter counter. Then the relevant volume of 10 generation culture was used to inoculate fresh $2 \mathrm{~mL} \mathrm{SC}$ containing the relevant treatment $(P$. insularum extract or control) to a final concentration of $5 \times 10^{6}$ cells $/ \mathrm{mL}$ and incubated at $30^{\circ} \mathrm{C}$ for $15 \mathrm{~h}$ (second 10 generations). After $15 \mathrm{~h}$, cell density was again determined using a Coulter counter, before cells were harvested for genomic DNA isolation. 


\subsubsection{Genomic DNA Isolation}

Genomic DNA (gDNA) was isolated using the YeaStar Genomic DNA Kit (ZymoResearch), according to Protocol I in the manufacturer's instructions. Briefly, $5 \times 10^{7}$ cells were harvested by centrifugation at $500 \times \mathrm{g}$ for $2 \mathrm{~min}$, the pellet was resuspended in $120 \mu \mathrm{L}$ of YD digestion buffer and 5 $\mu \mathrm{L}$ of R-Zymolase by vortexing, and the tubes were incubated at $37^{\circ} \mathrm{C}$ for $40-60 \mathrm{~min}$. Then $120 \mu \mathrm{L}$ of lysis buffer was added, followed by gentle mixing by inversion, and $250 \mu \mathrm{L}$ of chloroform was then added, followed by mixing for $1 \mathrm{~min}$. Tubes were then centrifuged at 10,000 rpm for $2 \mathrm{~min}$, and the upper layer (gDNA) was transferred onto a Zymo spin III column. The gDNA was bound to the column, the liquid phase was removed by centrifugation at 10,000 rpm for $1 \mathrm{~min}$, and $300 \mu \mathrm{L}$ of DNA wash buffer was added and removed twice by centrifugation at 10,000 rpm for $1 \mathrm{~min}$. The Zymo column was then transferred into a sterile $1.5 \mathrm{~mL}$ centrifuge tube and the gDNA was eluted with 60 $\mu \mathrm{L}$ sterile $\mathrm{dH}_{2} \mathrm{O}$ onto the membrane, incubated for 1 min at room temperature, and centrifuged at $10,000 \mathrm{rpm}$ for $10 \mathrm{~s}$ to yield $10-20 \mu \mathrm{g}$ of gDNA.

\subsubsection{Barcode Sequencing}

The unique 20 bp barcodes flanking each gene deletion (UPTAG upstream and DNTAG downstream of each gene deletion) were amplified and sequenced as previously described (Robinson et al., 2014). In brief, $10 \mathrm{ng} / \mathrm{uL}$ of gDNA was used as a template in a PCR using universal primers that are upstream and downstream of every barcode. All UPTAG amplicon libraries were pooled separately from all the DNTAG amplicon libraries, and $60 \mathrm{ng}$ of each pool underwent PCR amplification for the incorporation of the Illumina P5 adapter sequence (primer sequences in Appendix VII). The multiplexed UPTAG and DNTAG libraries were mixed together at a $1: 1$ ratio and $50 \mathrm{bp}$ single end reads were sequenced on an Illumina flow cell using an IlluminaHiSeq 2000 platform. Read matching and statistical analyses were carried out as previously described to record logFC (measure of change in mutant barcode sequence abundance in treatment compared to 
control), FDR (false discovery rate) and logCPM (quality of barcode sequencing results) for every gene deletion in the HET and HOM diploid libraries (Robinson et al., 2014).

\subsubsection{Validation of Fitness Phenotype}

Heterozygous or homozygous strains that were selected for validations were arrayed and grown in 96-format on YPD agar at $30^{\circ} \mathrm{C}$ overnight, which was subsequently used to inoculate a fresh 96-well plate containing $100 \mu \mathrm{L}$ of YPD and incubated at $30^{\circ} \mathrm{C}$ overnight. Cells were centrifuged, washed, and resuspended in $100 \mu \mathrm{L}$ of $\mathrm{dH}_{2} \mathrm{O}$, of which $1 \mu \mathrm{L}$ was used to inoculate fresh SC containing the relevant amount of extract or control. All validations and controls were carried out in triplicate and grown at $30^{\circ} \mathrm{C}$. Absorbance readings at $590 \mathrm{~nm}$ using an Envision plate reader (Perkin Elmer) were recorded at $0,15,18$ and $24 \mathrm{~h}$. Growth comparisons of treated and control cells were conducted to quantify growth inhibition and determine the heterozygous and homozygous deletion strains that were sensitive to $P$. insularum extracts. 


\subsection{Results}

\subsection{1. $\quad$ HIP Analysis of the P. insularum Aqueous Extract}

To determine the haploinsufficiency profile (HIP) of S. cerevisiae in the presence of the aqueous extract of $P$. insularum, the pooled heterozygous library was exposed to the aqueous extract $(0.001 \% \mathrm{v} / \mathrm{v})$, and grown for 20 generations. The gDNA was harvested and the barcodes flanking each gene deletion were amplified, sequenced and quantified using next-generation sequencing and bioinformatics analyses. Comparison of the growth profiles of the heterozygous mutants in the presence and absence of the aqueous extract resulted in calculations of logCPM and logFC. In our analyses, logCPM is the log counts per million, and is a measure of the reliability of the results. If an ORF does not produce sufficient reads/counts, it is discounted from the analyses. Conversely, $\operatorname{logFC}$ is the log fold change, and is a measure of the effect of the extract treatment. In this work, a negative logFC correlated to a reduction in read counts from ORFs treated with the extract compared to those without extract treatment, indicative of hypersensitivity and reduced growth of a particular deletion strain in the presence of the extract. A positive logFC correlated to an increase in read counts from ORFs treated with the extract compared to those without extract treatment, indicative of improved growth when treated with extract. Of the 5,814 heterozygous mutants, the majority exhibited logCPM greater than 3 indicative of a high quality of sequencing (Fig 4.1). While a $\operatorname{logFC}$ value at 0 indicates the treated heterozygous strain grew comparatively to the control, positive logFC values indicate the treated heterozygous mutants grew better than the control and negative logFC values show the treated heterozygous mutants grew worse than the control and are hypersensitive to the extract treatment. Using a False Discovery Rate (FDR) cutoff of $<0.05,26$ strains with identified with positive $\operatorname{logFC}$ and 25 strains with negative logFC. When the 51 genes were analysed through GO enrichment, no biological processes, molecular functions or cellular components were enriched.

The 25 heterozygous deletions that exhibited hypersensitivity (negative logFC) to the aqueous extract of $P$. insularum treatment are involved in gene expression at the genomic, 
transcription and translation stages, as well as trafficking in the cell involving the ER, the Golgi and the vacuole (Table 4.1). Interestingly, the iron-transporter FET4 was identified as one of the most hypersensitive genes in the HIP analysis, a result that is consistent with our previous finding that the FET4-deficient haploid deletion strain was hypersensitive to the extracts of $P$. insularum (Chapter 3 ).

There were 26 heterozygous strains that exhibited better growth in the presence of the $P$. insularum aqueous extract compared to the control (Table 4.2). Heterozygous mutations that conferred better growth included genes involved in histone structure, autophagy, and cell trafficking involving the Golgi. Of most interest from this set of results is the better growth profile of FRA1, (Fe Repressor of Activation) which is a negative regulator of the iron regulon.

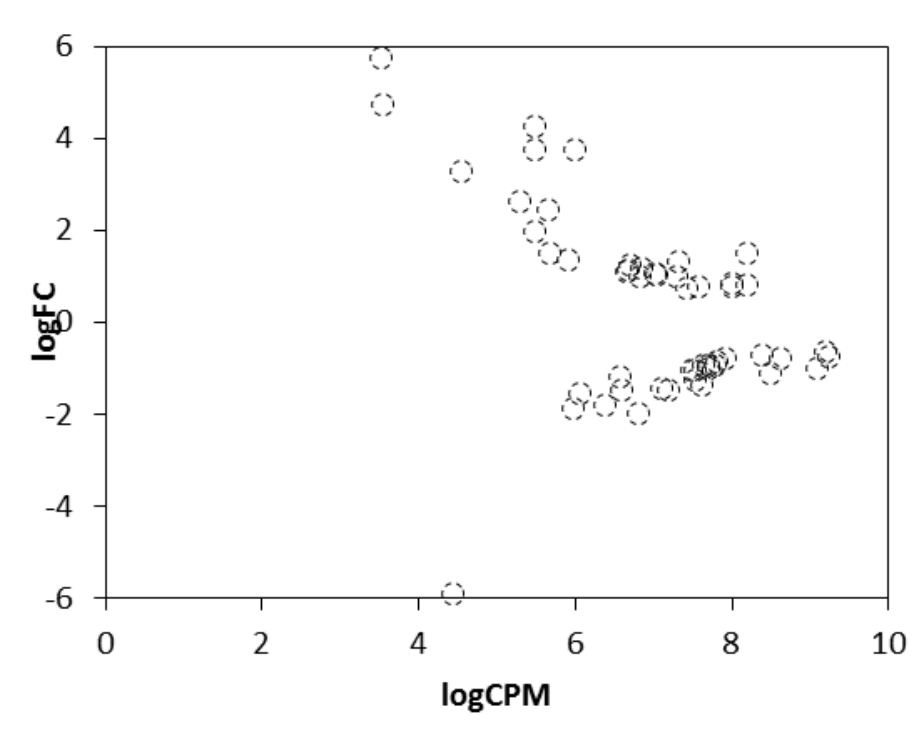

Figure 4.1: Scatterplot of heterozygous mutants with false discovery rate values less than 0.05 against the aqueous extract of $P$. insularum, plotted as $\operatorname{logCPM}$ vs $\operatorname{logFC}$. Read count of treated mutants were compared to read count of control mutants and is represented as the logFC, whereby $\operatorname{logFC}$ 0 indicates no change/difference between control and treated strains, while a positive or negative $\operatorname{logFC}$ indicates that treated heterozygotes grew better or worse (respectively) compared to the control. The plotted $\log$ CPM is a measure of data quality, whereby higher logCPM (e.g., greater than 2$)$ is considered good quality data. 
Table 4.1: The 25 hypersensitive heterozygotes against the aqueous extract of $\boldsymbol{P}$. insularum. The data summarized herein are sorted by the logFC, with the lowest $\operatorname{logFC}$ at the top of the table, indicating the genes are sorted from most hypersensitive to the least hypers ensitive true hits. logCPM, p-value and FDR values for all gene hits are shown, together with their gene names and biological functions obtained from the Saccharomyces Gene Database.

\begin{tabular}{|c|c|c|c|c|c|c|}
\hline & $\operatorname{logFC}$ & $\log \mathrm{CPM}$ & PValue & FDR & Gene & Biological Function \\
\hline YER157W & -5.89255 & 4.425087 & 7.07E-05 & $2.25 \mathrm{E}-02$ & COG3 & Essential component of the conserved oligomeric Golgi complex \\
\hline YMR319C & -1.97577 & 6.788245 & 7.23E-05 & $2.25 \mathrm{E}-02$ & FET4 & Low affinity iron transporter at the plasma membrane \\
\hline YJL172W & -1.84687 & 5.951847 & $6.20 \mathrm{E}-05$ & $2.25 \mathrm{E}-02$ & CPS1 & Vacuolar carboxy peptidase S \\
\hline YPL260W & -1.78916 & 6.365672 & $9.43 \mathrm{E}-06$ & $8.68 \mathrm{E}-03$ & YPL260W & Putative substrate of cAMP-dependent protein kinase \\
\hline YAL007C & -1.54478 & 6.046772 & $1.52 \mathrm{E}-04$ & $3.19 \mathrm{E}-02$ & ERP2 & Member of the p24 family involved in ER to Golgi transport \\
\hline YLR315W & -1.45443 & 7.183376 & $1.18 \mathrm{E}-05$ & 8.78E-03 & NKP2 & Central kinetochore protein and subunit of the Ctf19 complex \\
\hline YOL069W & -1.44614 & 6.574941 & 3.57E-04 & $4.82 \mathrm{E}-02$ & NUF2 & Component of the kinetochore-associated Ndc80 complex \\
\hline YCR028C & -1.40387 & 7.084485 & $1.64 \mathrm{E}-04$ & $3.25 \mathrm{E}-02$ & FEN2 & Plasma membrane $\mathrm{H}+$-pantothenate symporter \\
\hline YGL207W & -1.34066 & 7.596771 & $2.08 \mathrm{E}-05$ & 1.37E-02 & SPT16 & Subunit of the heterodimeric FACT complex \\
\hline YEL054C & -1.22951 & 7.527092 & $1.15 \mathrm{E}-04$ & $2.84 \mathrm{E}-02$ & RPL12A & Ribosomal 60S subunit protein L12A \\
\hline YCR020W-B & -1.18159 & 6.560867 & $1.56 \mathrm{E}-04$ & $3.19 \mathrm{E}-02$ & HTL1 & Component of the RSC chromatin remodeling complex \\
\hline YJL211C & -1.07773 & 8.472511 & $1.19 \mathrm{E}-09$ & 7.07E-06 & YJL211C & Dubious open reading frame, overlaps with PEX2 \\
\hline YPL021W & -1.02942 & 7.511751 & $7.85 \mathrm{E}-05$ & $2.33 \mathrm{E}-02$ & ECM23 & Non-essential protein of unconfirmed function \\
\hline YJL190C & -1.00397 & 7.478848 & $3.50 \mathrm{E}-04$ & $4.82 \mathrm{E}-02$ & RPS22A & Protein component of the small (40S) ribosomal subunit \\
\hline YML104C & -0.99336 & 7.691982 & $5.22 \mathrm{E}-05$ & $2.22 \mathrm{E}-02$ & MDM1 & PtdIns-3-P binding protein that tethers the ER to vacuoles at NVJs \\
\hline YHL027W & -0.96755 & 9.066119 & $6.56 \mathrm{E}-05$ & $2.25 \mathrm{E}-02$ & RIM101 & Cys2His2 zinc-finger transcriptional repressor \\
\hline YLR068W & -0.91841 & 7.770453 & $1.53 \mathrm{E}-04$ & 3.19E-02 & FYV7 & Essential protein required for maturation of $18 \mathrm{~S}$ rRNA \\
\hline YOR340C & -0.91718 & 7.622687 & 2.19E-04 & $3.71 \mathrm{E}-02$ & RPA43 & RNA polymerase I subunit A43 \\
\hline YCR102W-A & -0.9145 & 7.691793 & $3.78 \mathrm{E}-04$ & $4.85 \mathrm{E}-02$ & YCR102W-A & Dubious open reading frame \\
\hline YNR068C & -0.82746 & 7.787097 & $2.11 \mathrm{E}-04$ & $3.71 \mathrm{E}-02$ & YNR068C & Putative protein of unknown function \\
\hline YMR220W & -0.77389 & 8.612453 & $4.01 \mathrm{E}-04$ & $4.85 \mathrm{E}-02$ & ERG8 & Phosphomevalonate kinase involved in ergosterol synthesis \\
\hline YLL044W & -0.76131 & 7.898162 & $2.30 \mathrm{E}-04$ & 3.79E-02 & YLL044W & Dubious open reading frame \\
\hline YDL240W & -0.7137 & 9.219504 & $8.90 \mathrm{E}-05$ & 2.39E-02 & LRG1 & GTPase-activating protein \\
\hline YNL044W & -0.69931 & 8.372763 & $1.84 \mathrm{E}-04$ & $3.52 \mathrm{E}-02$ & YIP3 & Protein localized to COPII vesicles \\
\hline YGL213C & -0.63938 & 9.175094 & $1.26 \mathrm{E}-04$ & $2.88 \mathrm{E}-02$ & SKI8 & Ski complex component and WD-repeat protein \\
\hline
\end{tabular}


Table 4.2: The 26 heterozygotes that grew better in the aqueous extract of $\boldsymbol{P}$. insularum. The data summarized herein are sorted by the logFC, with the lowest $\log \mathrm{FC}$ at the top of the table, indicating the genes are sorted from least resistant to the most resistant to the aqueous extract of $P$. insularum. logCPM, p-value and FDR values for all gene hits are shown, together with their gene names and biological functions obtained from the Saccharomyces Gene Database. NA: no associated gene, simply an identified ORF.

\begin{tabular}{|c|c|c|c|c|c|c|}
\hline & $\log \mathrm{FC}$ & $\log \mathrm{CPM}$ & PValue & FDR & Gene & Biological Function \\
\hline YDL170W & 0.779259 & 7.561222 & $9.28 \mathrm{E}-05$ & 2.39E-02 & UGA3 & Transcriptional activator for GABA (gamma-aminobutyrate) genes \\
\hline YOR304C-A & 0.802629 & 7.981128 & $3.08 \mathrm{E}-04$ & $4.68 \mathrm{E}-02$ & BIL1 & Protein that binds Bud6p, role in actin cable assembly \\
\hline YLR150W & 0.830896 & 8.188551 & $1.02 \mathrm{E}-05$ & 8.68E-03 & STM1 & Protein required for optimal translation under nutrient stress \\
\hline YLR262C-A & 0.877725 & 7.993725 & 3.87E-04 & $4.85 \mathrm{E}-02$ & TMA7 & Unknown function, associates with ribosomes \\
\hline YDL134C-A & 1.009552 & 6.809188 & $3.36 \mathrm{E}-04$ & $4.82 \mathrm{E}-02$ & NA & NA \\
\hline YLL029W & 1.013686 & 7.273951 & 3.07E-04 & 4.68E-02 & FRA1 & Involved in negative regulation of iron regulon transcription \\
\hline YGR071C & 1.050189 & 7.035567 & $5.61 \mathrm{E}-05$ & $2.22 \mathrm{E}-02$ & ENV11 & Protein proposed to be involved in vacuolar functions \\
\hline YIL005W & 1.100702 & 7.00332 & $2.82 \mathrm{E}-04$ & $4.52 \mathrm{E}-02$ & EPS1 & ER protein with chaperone and co-chaperone activity \\
\hline YNL051W & 1.133584 & 6.636807 & $2.13 \mathrm{E}-04$ & $3.71 \mathrm{E}-02$ & COG5 & Component of the conserved oligomeric Golgi complex \\
\hline YFL014W & 1.175569 & 6.663223 & 3.57E-04 & 4.82E-02 & HSP12 & Plasma membrane protein involved in maintaining membrane organization \\
\hline YPR026W & 1.186516 & 6.824689 & $1.02 \mathrm{E}-05$ & 8.68E-03 & ATH1 & Acid trehalase required for the utilization of extracellular trehalose \\
\hline YBL002W & 1.2581 & 6.675206 & 2.09E-04 & $3.71 \mathrm{E}-02$ & HTB2 & Histone $\mathrm{H} 2 \mathrm{~B}$ \\
\hline YOL019W & 1.34473 & 7.306941 & $5.42 \mathrm{E}-05$ & $2.22 \mathrm{E}-02$ & YOL019W & Protein of uknown function \\
\hline YOR365C & 1.361513 & 5.897571 & $1.24 \mathrm{E}-04$ & $2.88 \mathrm{E}-02$ & YOR365C & Putative protein of unknown function \\
\hline YPR049C & 1.512557 & 5.667632 & 3.99E-04 & 4.85E-02 & ATG11 & Adapter protein for pexophagy and the Cvt targeting pathway \\
\hline YNL062C & 1.523893 & 8.168126 & $1.20 \mathrm{E}-06$ & $2.86 \mathrm{E}-03$ & GCD10 & Subunit of the tRNA methyltransferase with Gcd14p \\
\hline YBR133C & 2.006621 & 5.468299 & 4.24E-04 & 4.93E-02 & HSL7 & Arginine N-methyltransferase \\
\hline YBL003C & 2.458397 & 5.633028 & $2.35 \mathrm{E}-06$ & $3.48 \mathrm{E}-03$ & HTA2 & Histone $\mathrm{H} 2 \mathrm{~A}$ \\
\hline YBL005W & 2.634583 & 5.273181 & $3.48 \mathrm{E}-05$ & 2.03E-02 & PDR3 & Transcriptional activator of the pleiotropic drug resistance network \\
\hline YPL158C & 3.295032 & 4.53157 & 4.19E-04 & 4.93E-02 & AIM44 & Regulates Cdc42p and Rho1p \\
\hline YFL033C & 3.757344 & 5.987579 & $1.45 \mathrm{E}-06$ & $2.86 \mathrm{E}-03$ & RIM15 & Protein kinase involved in cell proliferation in response to nutrients \\
\hline YPR021C & 3.778685 & 5.467699 & 4.07E-05 & 2.03E-02 & AGC1 & Mitochondrial amino acid transporter \\
\hline YDR506C & 4.265256 & 5.474882 & $4.11 \mathrm{E}-05$ & 2.03E-02 & GMC1 & Involved in meiotic progression \\
\hline YEL072W & 4.765103 & 3.5177 & 3.93E-04 & 4.85E-02 & RMD6 & Required for sporulation \\
\hline YGR062C & 5.773421 & 3.49702 & 8.57E-05 & 2.39E-02 & CoX18 & Required for membrane insertion of C-terminus of $\operatorname{Cox} 2 p$ \\
\hline
\end{tabular}


As false positives are inherent in high throughput analyses such as the genome-wide Barseq analyses conducted in this chapter, results of such analyses must be validated using independent methods. We previously focussed on the haploid mutants with impaired growth in the presence of the extracts (in Chapter 3 ) and validation work in this chapter also only investigates the validity of diploid mutants on that basis. We thus grew the 25 heterozygous strains with negative logFC in the presence of the aqueous extract or the control treatment, and measured their growth (absorbance at $590 \mathrm{~nm}$ ) at $15 \mathrm{~h}$, a time point where yeast strains are at mid-log phase of growth. Growth (percent residual growth) of six of the 25 strains was significantly reduced by the aqueous $P$. insularum treatments compared to the BY4743 WT control (Fig 4.2). These mutants were Fet4p (the lowaffinity iron transporter), Cps1p (the vacuolar caboxypeptidase S), Lrg1p (a GTPAse activating protein), Yip3p (a protein localised to COP II vesicles), and two uncharacterized genes YPL260W and YNR068C (Bordallo et al., 1991; Dix et al., 1994; Fitch et al., 2004; Genget al., 2005).

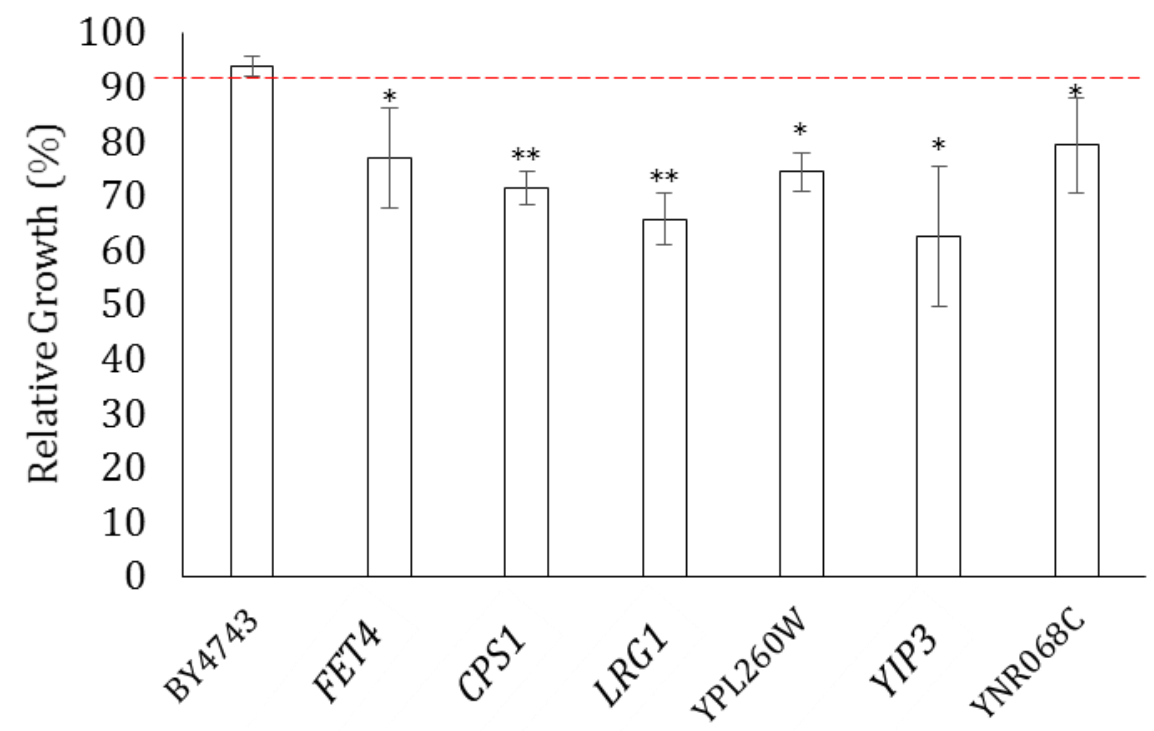

Figure 4.2: Heterozygous genes validated to be hypersensitive to the aqueous extract of $P$. insularum. Heterozygous mutants were individually grown in the presence of the aqueous extract in triplicate, and compared to the control treatment. Mutants exhibiting growth less than $90 \%$ consistently across the three triplicates compared to the WT (BY4743) are plotted as residual growth as mean \pm SD. The p-values were calculated using one tailed Student's t-test $\left({ }^{*}\right.$ : p-value $<0.05,{ }^{* *}$ : p-value $\left.<0.01\right)$. 


\subsubsection{HOP Analysis of the P. insularum Aqueous Extract}

To determine the homozygous profile of the aqueous extract of $P$. insularum, the pooled collection of homozygous deletion mutants was exposed to $0.001 \% \mathrm{v} / \mathrm{v}$ of the aqueous extract and the growth profiles (barcode abundance) of each deletion mutant in the presence of the extract was compared to their control counterparts. The logFC and $\log C P M$ values for each deletion mutant were determined and the majority of the deletion mutants were largely unaffected by the extract treatment with most of the ORFs scattered around a logFC value of 0 (Fig 4.3). Using FDR $<0.05$ as a cutoff for significance, there were 18 deletion mutants with negative logFC values ranging from 0.5 to -5.5 (Table 4.3) indicative of impaired growth in the presence of the extract, and 12 deletion mutants with positive logFC values ranging from 0.3 to 3.5 (Table 4.4) indicating better growth in the presence of the extract. When the 30 genes were analysed through GO enrichment, no biological processes, molecular functions or cellular components were enriched.

The 18 genes with negative logFC values function in a variety of cellular processes (Table 4.2). The gene deletion most hypersensitive (i.e., the lowest $\operatorname{logFC}$ value) to the aqueous extract treatment was ERG2, an enzyme involved in ergosterol synthesis (Arthington et al., 1991). Interestingly, the ergosterol synthesis pathway requires the co-factor heme at the Erg11p step of the pathway (Turi \& Loper, 1992). Several genes can be observed as belonging to distinct molecular components and functions. For instance, several gene deletions involved in ribosomal structure were detected (e.g.,rpp1a $, r p / 37 a \Delta, r p s 4 b \Delta, j j j 1 \Delta, r p / 17 b \Delta$ and $r p / 4 a \Delta)$ (Cyr et al., 1994; Planta \& Mager, 1998; Stolc \& Altman, 1997). Several gene deletions were determined to be involved in

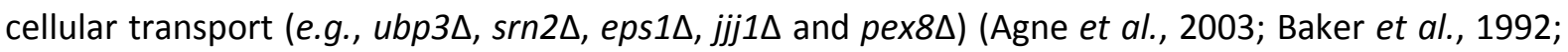
Robinson et a., 1988; Wang \& Chang, 1999). Two deletion mutants were observed to regulate transcription of specific genes and pathways, including $m t q 2 \Delta$ and fyv5 (Bianchi et a., 1999; Polevodaet al., 2006). Dubious ORFs and putative proteins were also detected, including yn/338w $y d 1242 w \Delta$ and yil001wA. 
The 12 gene deletions that showed improved growth exhibited varied functions (Table 4.4). Some are involved in the pleiotropic drug response (PDR3), autophagy (ATG11), oxidative stress (YAP1) and protein breakdown (PNG1). Interestingly, the null deletion mutant of ROX1, a hemedependent repressor of hypoxic genes, exhibited improved growth in the presence of the aqueous extract.

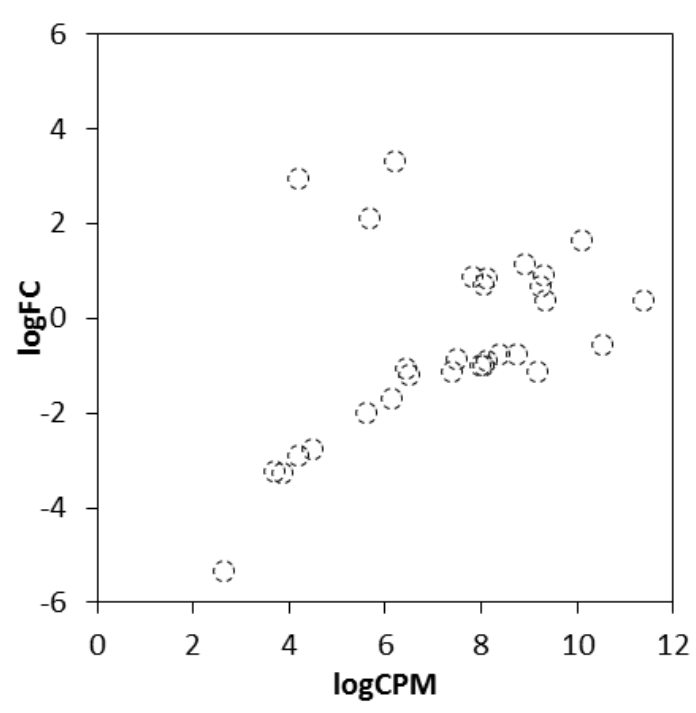

Figure 4.3: Scatterplot of the $\mathbf{3 0}$ homozygous deletion mutants with false discovery rate values less than 0.05 against the aqueous extract of $P$. insularum, plotted as $\operatorname{logCPM}$ vs $\log$. Pooled homozygous mutants were treated with $0.001 \% \mathrm{v} / \mathrm{v}$ aqueous extract of $P$. insularum and exposed to a total of 20 generations, DNA extracted and barcode sequenced. Charted are deletion mutants with FDR values calculated to be less than 0.05 , plotted using logCPM vs logFC. 
Table 4.3: $\quad$ The 18 hypersensitive homozygous deletion mutants against the aqueous extract of $P$. insularum. The data summarized herein are sorted by the $\log \mathrm{FC}$, with the lowest $\log \mathrm{FC}$ at the top of the table, indicating the genes are sorted from most hypersensitive to the least hypersensitive true hits. logCPM, pvalue and FDR values for all gene hits are shown, together with their gene names and biological functions obtained from the Saccharomyces Gene Database.

\begin{tabular}{|c|c|c|c|c|c|c|}
\hline & $\log \mathrm{FC}$ & $\log \mathrm{CPM}$ & PValue & FDR & Gene & Biological Function \\
\hline YMR202W & -5.32403 & 2.627291 & $1.58 \mathrm{E}-04$ & $3.60 \mathrm{E}-02$ & ERG2 & C-8 sterol isomerase, involved in ergosterol synthesis \\
\hline YDR140W & -3.25781 & 3.847427 & $7.41 \mathrm{E}-05$ & $2.44 \mathrm{E}-02$ & MTQ2 & S-adenosylmethionine-dependent methyltransferase \\
\hline YER151C & -3.22246 & 3.67191 & $1.06 \mathrm{E}-04$ & $3.13 \mathrm{E}-02$ & UBP3 & Ubiquitin-specific protease involved in transport and osmotic response \\
\hline YCL058C & -2.87062 & 4.177405 & $8.70 \mathrm{E}-06$ & 7.04E-03 & FYV5 & Protein involved in regulation of the mating pathway \\
\hline YDL081C & -2.73501 & 4.471486 & $1.32 \mathrm{E}-04$ & $3.26 \mathrm{E}-02$ & RPP1A & Ribosomal stalk protein P1 alpha \\
\hline YLR185W & -1.96176 & 5.587265 & 1.57E-06 & $1.86 \mathrm{E}-03$ & RPL37A & Ribosomal 60S subunit protein L37A \\
\hline YHR203C & -1.66012 & 6.116239 & 4.32E-05 & 1.97E-02 & RPS4B & Protein component of the small (40S) ribosomal subunit \\
\hline YLR119W & -1.18286 & 6.46769 & $1.46 \mathrm{E}-04$ & $3.46 \mathrm{E}-02$ & SRN2 & Component of the ESCRT-I complex \\
\hline YIL005W & -1.115 & 9.141387 & $2.31 \mathrm{E}-04$ & 4.57E-02 & EPS1 & ER protein with chaperone and co-chaperone activity \\
\hline YOR275C & -1.11443 & 7.373076 & $2.01 \mathrm{E}-04$ & $4.26 \mathrm{E}-02$ & RIM20 & Protein involved in proteolytic activation of Rim101p; part of response to alkaline $\mathrm{pH}$ \\
\hline YNL227C & -1.02762 & 6.398809 & $1.21 \mathrm{E}-04$ & 3.13E-02 & JJJ1 & Co-chaperone that stimulates the ATPase activity of Ssa1p, ribosome biogenesis \\
\hline YJL177W & -0.98441 & 8.021737 & $5.66 \mathrm{E}-05$ & $2.24 \mathrm{E}-02$ & RPL17B & Ribosomal 60S subunit protein L17B \\
\hline YNL338W & -0.97276 & 7.958258 & $1.16 \mathrm{E}-04$ & 3.13E-02 & YNL338W & Dubious open reading frame \\
\hline YKR027W & -0.88167 & 8.102546 & $1.07 \mathrm{E}-06$ & $1.58 \mathrm{E}-03$ & $\mathrm{BCH} 2$ & Member of the ChAPs (Chs5p-Arf1p-binding proteins) family \\
\hline YBR031W & -0.85451 & 7.458257 & $6.14 \mathrm{E}-05$ & 2.27E-02 & RPL4A & Ribosomal 60S subunit protein L4A \\
\hline YGR077C & -0.7311 & 8.359912 & $1.71 \mathrm{E}-04$ & $3.75 \mathrm{E}-02$ & PEX8 & Intraperoxisomal organizer of the peroxisomal import machinery \\
\hline YDL242W & -0.72361 & 8.735494 & $9.50 \mathrm{E}-06$ & $7.04 \mathrm{E}-03$ & YDL242W & Dubious open reading frame \\
\hline YIL001W & -0.53614 & 10.49215 & $2.29 \mathrm{E}-04$ & 4.57E-02 & YIL001W & Putative protein of unknown function \\
\hline
\end{tabular}


Table 4.4: $\quad$ The 12 homozygous gene deletions that grew better in the presence of the aqueous $P$. insularum extract. The data summarized herein are sorted by the $\operatorname{logFC}$, with the lowest logFC at the top of the table, indicating the genes are sorted from least resistant to the most resistant to the aqueous extract of P. insularum. $\log$ CPM, p-value and FDR values for all gene hits are shown, together with their gene names and biological functions obtained from the Saccharomyces Gene Database.

\begin{tabular}{|c|c|c|c|c|c|c|}
\hline & $\operatorname{logFC}$ & $\log \mathrm{CPM}$ & PValue & FDR & common & Function \\
\hline YFL033C & 0.391197 & 11.37304 & $2.78 \mathrm{E}-05$ & $1.50 \mathrm{E}-02$ & RIM15 & Protein kinase involved in cell proliferation in response to nutrients \\
\hline YBL005W & 0.404761 & 9.318519 & $1.18 \mathrm{E}-04$ & 3.13E-02 & PDR3 & Transcriptional activator of the pleiotropic drug resistance network \\
\hline YLR150W & 0.698048 & 9.211196 & $6.96 \mathrm{E}-05$ & $2.43 \mathrm{E}-02$ & STM1 & Protein required for optimal translation under nutrient stress \\
\hline YLR187W & 0.740099 & 8.034502 & 8.89E-05 & $2.77 \mathrm{E}-02$ & SKG3 & Protein of unknown function \\
\hline YPL096W & 0.857543 & 8.109192 & $3.84 \mathrm{E}-07$ & $7.58 \mathrm{E}-04$ & PNG1 & Conserved peptide N-glycanase \\
\hline YPR049C & 0.894325 & 7.810629 & $2.98 \mathrm{E}-06$ & $2.95 \mathrm{E}-03$ & ATG11 & Adapter protein for pexophagy and the Cvt targeting pathway \\
\hline YPR064W & 0.919732 & 9.279071 & $3.06 \mathrm{E}-05$ & $1.51 \mathrm{E}-02$ & YPR064W & Dubious ORF \\
\hline YML007W & 1.173474 & 8.876484 & $2.77 \mathrm{E}-05$ & $1.50 \mathrm{E}-02$ & YAP1 & Transcription factor required for oxidative stress tolerance \\
\hline YDL225W & 2.135633 & 5.651854 & $1.25 \mathrm{E}-05$ & 8.23E-03 & SHS1 & Component of the septin ring required for cytokinesis \\
\hline YDR401W & 2.984504 & 4.179569 & $4.96 \mathrm{E}-05$ & $2.10 \mathrm{E}-02$ & YDR401W & Dubious ORF \\
\hline YDR392W & 3.34067 & 6.188196 & $9.27 \mathrm{E}-10$ & $2.75 \mathrm{E}-06$ & SPT3 & Subunit of the SAGA transcriptional regulatory complexes \\
\hline
\end{tabular}


To validate the results of the Bar-seq analysis, we grew the 18 homozygous gene deletion mutants with negative logFC values (between -0.5 and -5.5 ) and FDR $<0.05$ in the presence and absence of the $P$. insularum aqueous extract and measured growth (absorbance 590nm) at $15 \mathrm{~h}$, a time point where cells were at mid-log of growth. The residual growth (treated/untreated $x$ 100) for each deletion mutant was calculated and then compared to that of the BY4743 WT (Fig 4.4). Seven homozygous deletion mutants were hypersensitive to the extract compared to the WT control. The most sensitive gene was the ergosterol synthesis gene ERG2 (Arthington et al., 1991), a result that is consistent with the ERG2 mutant being the most sensitive strain in the Bar-seq analyses. Also sensitive were JJJ1, RPL4b and RPS4B that are all involved in ribosomal biogenesis (Planta \& Mager, 1998; Walsh et al., 2004), the dubious ORFs YGR151C and YDL242W, and the putative protein encoded for by YILOOTW.

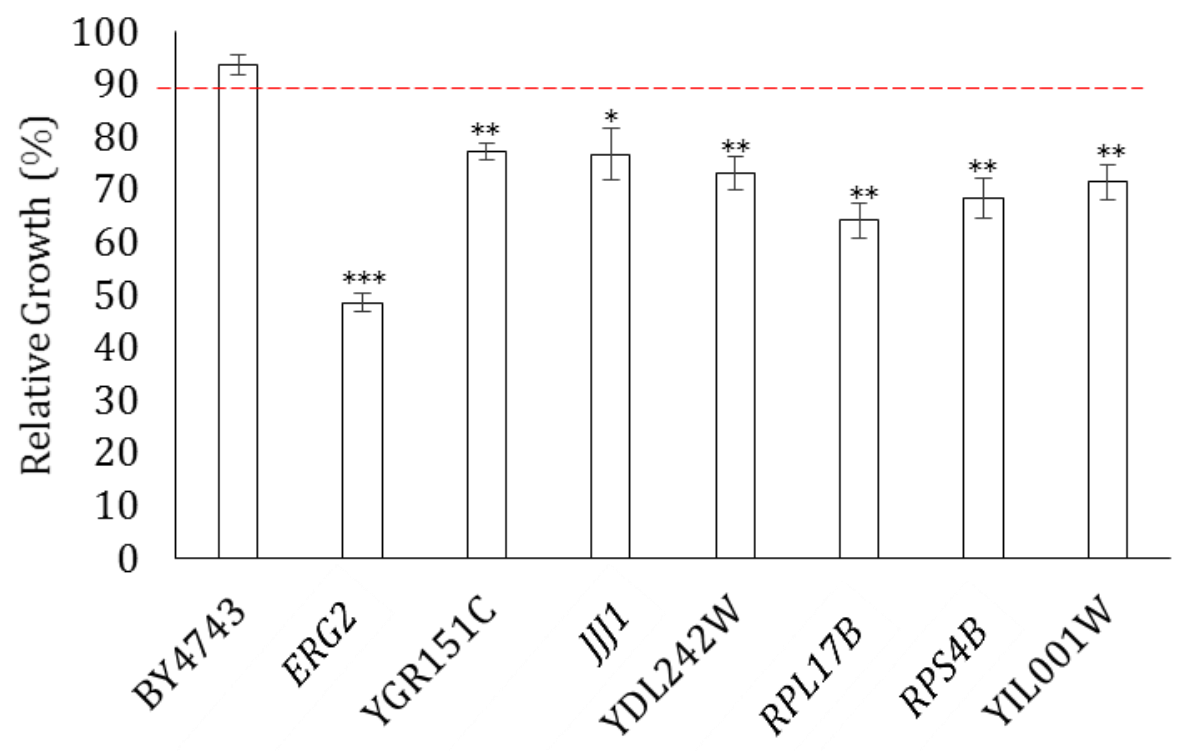

Figure 4.4: Validations of the homozygous gene deletions detected to be hypersensitive to the aqueous extract of $\boldsymbol{P}$. insularum. Homozygous deletion mutants were individually grown in the presence of the aqueous extract in triplicate, and compared to the control treatment. Mutants exhibiting growth less than $90 \%$ consistently across the three triplicates compared to the WT (BY4743) are plotted as mean residual growth $\pm \mathrm{SD}$. The p-values were calculated using a one tailed Student's t-test $(*$ : p-value $<0.05,{ }^{* *}$ : p-value $<0.01,{ }^{* * *}$ : p-value $\left.<0.001\right)$. 


\subsubsection{HIP Analysis of the P. insularum Methanolic Extract}

As the priority aim of this thesis is primarily gaining understanding of the biological and chemical mechanisms of action of $P$. insularum as it is prepared by traditional healers in Samoa, it was only the aqueous extract that was used in the agar-based genome-wide analyses in Chapter 3. To explore the haploinsufficiency profile of the methanolic extract of $P$. insularum, the pooled heterozygous library was grown in the presence and absence of $0.0001 \% \mathrm{v} / \mathrm{v}$ of the methanolic extract. Similar to the previous Bar-seq results collected for the aqueous $P$. insularum extract, the majority of the mutant strains showed logFC values around 0 , indicating that the $P$. insularum methanolic extract did not affect growth compared to the control (Fig 4.5). Using FDR $<0.05$ as a cutoff for significance, there were 20 heterozygous mutants that exhibited impaired growth in the presence of the methanolic extract compared to the control, with negative logFC values ranging from -0.75 to -2.65 . On the same basis, there were 31 heterozygous mutants that grew better in the presence of the methanolic extract compared to the control, with logFC values ranging from 0.75 to 1.65. When the 51 genes detected here were analysed for GO enrichment, no enrichment was detected from biological processes, molecular functions or cellular components.

Of the 20 genes with negative logFC with FDR $<0.05$ (Table 4.5), the heterozygous ORF shown to be the most hypersensitive with the lowest logFC was IRC7, which encodes a $\beta$-lyase that exhibits increased expression in response to copper levels (Gross et al., 2000; Roncoroni et al., 2011). Several ORFs were observed to be clearly implicated in specific pathways. For instance, RPL10, RPL24B and RPS29A are involved in ribosomal structure (Planta \& Mager, 1998), while SWC5, SPT16, ITC1 and SET5 are involved in chromatin remodelling (Krogan et al., 2003; Malone et al., 1991; Nislow et al., 1997; Sugiyama \& Nikawa, 2001). Interestingly, HEM2 that encodes aminolevulinate dehydratase, an enzyme involved in heme synthesis (Gollub et al., 1977), was also detected. Several other ORFs involved in autophagy (ATG15), cell cycle (BUB1 and SFI1), transcriptional regulation (CDC39), translational control (SBP1), as well as proteins found at the plasma membrane (STL1) and the cell wall (FLO11) were recovered (Breter et al., 1983; Hoyt et al., 
1991; Jong et al., 1986; Klonsky et al., 2003; Lo \& Dranginis, 1996; Ma et al., 1999; Zhao et al., 1994). Dubious ORFs and putative proteins of unknown functions were also detected, including YAR043C and YKLO36C.

The 31 heterozygous mutants that grew better in the presence of the methanolic extract of P. insularum were involved in a variety of functions (Table 4.6), a few of which were intriguing. For instance, STM1 required for optimal translation under nutrient stress (van Dyke et al., 2006) and BIL1 which binds Bud6p and is involved in actin cable assembly (Graziano et al., 2013) were detected in the HIP of the aqueous extract of $P$. insularum, were also detected in the HIP of the methanolic extract. Other genes were detected from pathways involving cellular transport and trafficking (MUK1, PEP12 and EXO84), mitochondrial ribosomal structure (RSM25 and MRPL22), transcriptional regulation (RSF2, SPN1 and SPT6), as well as protein recycling and degradation (UBP6, CDC34 and HRD3) (Carbrera et al., 2013; Gracck \& Wittmann-Lliebold, 1998; Gardener et al., 2000;Goebl et al., 1988; Guo et al., 1999; Krogan et al., 2002; Park et al., 1997;Rothman \& Stevens, 1986; Saveanu et al., 2001; Tkach et al., 2012; Youdell et al., 2008).

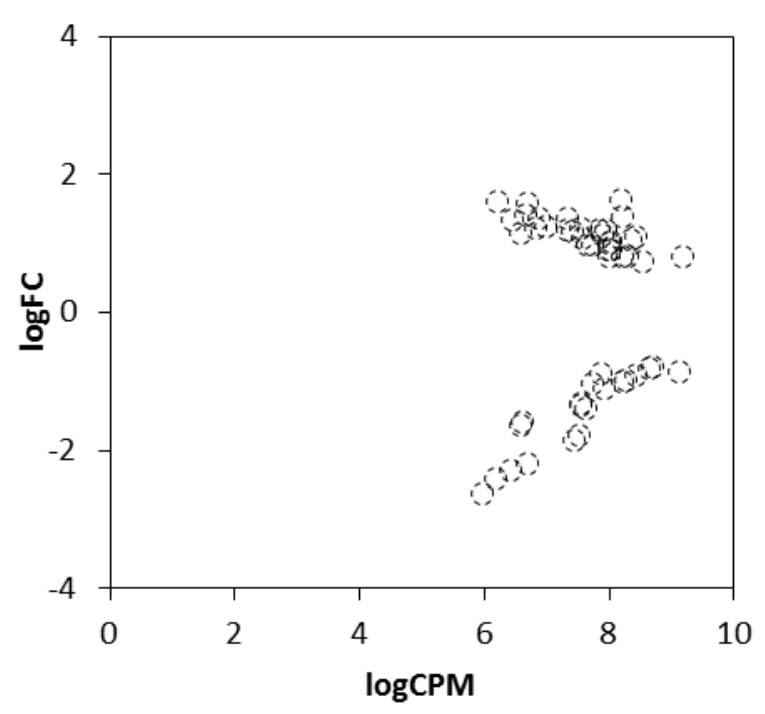

Figure 4.5: Scatterplot of the $\mathbf{5 1}$ heterozygous mutants with false discovery rate values less than 0.05 against the methanolic extract of $P$. insularum, plotted as $\operatorname{logCPM}$ vs $\operatorname{logFC}$. Pooled heterozygous mutants were treated with $0.0001 \% \mathrm{v} / \mathrm{v}$ of methanolic extract over 20 generations, DNA extracted and barcode sequenced. The mutants with FDR $<0.05$, are plotted using logCPM against $\log F C$. 
Table 4.5: The 20 hypersensitive heterozygotes resulting from treatment with the methanol extract of $P$. insularum. The data summarized herein are sorted by the $\log \mathrm{FC}$, with the lowest $\log \mathrm{FC}$ at the top of the table, indicating the genes are sorted from most hypersensitive to the least hypersensitive true hits. $\log \mathrm{CPM}$, p-value and FDR values for all gene hits are shown, together with their gene names and biological functions obtained from the Saccharomyces Gene Database.

\begin{tabular}{|c|c|c|c|c|c|c|}
\hline & $\operatorname{logFC}$ & $\log \mathrm{CPM}$ & PValue & FDR & Gene & Biological Function \\
\hline YFR055W & -2.60733 & 5.959965 & $2.74 \mathrm{E}-05$ & 0.010818 & IRC7 & Beta-lyase involved in the production of thiols \\
\hline YHL034C & -2.39046 & 6.17154 & $1.92 \mathrm{E}-05$ & 0.009467 & SBP1 & Protein that binds eIF4G and has a role in repression of translation \\
\hline YAR043C & -2.29016 & 6.416529 & $2.20 \mathrm{E}-04$ & 0.040517 & YAR043C & Does not encode a protein \\
\hline YBR281C & -2.18888 & 6.670253 & $5.70 \mathrm{E}-05$ & 0.016075 & DUG2 & Component of glutamine amidotransferase (GATase II) \\
\hline YBR231C & -1.84337 & 7.406921 & 4.19E-07 & 0.001242 & SWC5 & Component of the SWR1 complex \\
\hline YCR068W & -1.76099 & 7.493509 & $2.17 \mathrm{E}-05$ & 0.009467 & ATG15 & Putative lipase required for lysis of autophagic and Cvt bodies \\
\hline YDR156W & -1.62516 & 6.570473 & $4.22 \mathrm{E}-04$ & 0.049541 & RPA14 & RNA polymerase I subunit A14 \\
\hline YLR075W & -1.58644 & 6.587466 & 8.90E-05 & 0.021095 & RPL10 & Ribosomal 60S subunit protein L10 \\
\hline YGL207W & -1.3877 & 7.596771 & $2.34 \mathrm{E}-04$ & 0.040719 & SPT16 & Subunit of the heterodimeric FACT complex (Spt16p-Pob3p) \\
\hline YGR148C & -1.32513 & 7.529734 & $4.21 \mathrm{E}-04$ & 0.049541 & RPL24B & Ribosomal 60S subunit protein L24B \\
\hline YCR093W & -1.30638 & 7.517605 & $4.26 \mathrm{E}-04$ & 0.049541 & CDC39 & Component of the CCR4-NOT1 core complex \\
\hline YGR188C & -1.09262 & 7.911316 & $1.28 \mathrm{E}-04$ & 0.027152 & BUB1 & Protein kinase involved in the cell cycle checkpoint into anaphase \\
\hline YLR388W & -1.02036 & 7.70285 & $2.26 \mathrm{E}-04$ & 0.040517 & RPS29A & Protein component of the small (40S) ribosomal subunit \\
\hline YIR019C & -0.98499 & 8.187611 & $5.28 \mathrm{E}-05$ & 0.016 & FL011 & GPI-anchored cell surface glycoprotein (flocculin) \\
\hline YKL036C & -0.9641 & 8.243288 & $1.90 \mathrm{E}-04$ & 0.03639 & YKL036C & Dubious open reading frame \\
\hline YDR536W & -0.90094 & 8.406403 & $2.40 \mathrm{E}-04$ & 0.040719 & STL1 & Glycerol proton symporter of the plasma membrane \\
\hline YGL040C & -0.88203 & 7.855504 & $4.05 \mathrm{E}-04$ & 0.049541 & HEM2 & Aminolevulinate dehydratase; a homo-octameric enzyme, heme synthesis \\
\hline YGL133W & -0.84329 & 9.108457 & $2.02 \mathrm{E}-05$ & 0.009467 & ITC1 & Subunit of ATP-dependent Isw2p-Itc1p chromatin remodeling complex \\
\hline YHR207C & -0.81185 & 8.620949 & $5.40 \mathrm{E}-05$ & 0.016 & SET5 & Methyltransferase involved in methylation of histone H4 Lys5, -8, -12 \\
\hline YLL003W & -0.77468 & 8.670692 & $2.93 \mathrm{E}-04$ & 0.044455 & SFI1 & Centrin (Cdc31p)-binding protein required for SPB duplication \\
\hline
\end{tabular}


Table 4.6: The 31 heterozygous gene deletions with positive logFC and FDR $<\mathbf{0 . 0 5}$. The data summarized herein are sorted by the logFC, with the lowest $\operatorname{logFC}$ at the top of the table, indicating the genes are sorted from least resistant to the most resistant to the methanolic extract of $P$. insularum. logCPM, p-value and FDR values for all gene hits are shown, together with their gene names and biological functions obtained from the Saccharomyces Gene Database.

\begin{tabular}{|c|c|c|c|c|c|c|}
\hline & $\operatorname{logFC}$ & $\log C P M$ & PValue & FDR & common & Function \\
\hline YHL019C & 0.8082392 & 9.15137 & $3.11 \mathrm{E}-04$ & 0.045973049 & APM2 & Protein of unknown function \\
\hline YLR150W & 0.8150514 & 8.188551 & $1.76 \mathrm{E}-04$ & 0.035772533 & STM1 & Protein required for optimal translation under nutrient stress \\
\hline YLR207W & 0.8223704 & 7.986241 & $7.66 \mathrm{E}-05$ & 0.018915263 & HRD3 & ER membrane protein that plays a central role in ERAD \\
\hline YOR084W & 0.8265312 & 8.28 & $1.19 \mathrm{E}-04$ & 0.026229726 & LPX1 & Peroxisomal matrix-localised lipase \\
\hline YJR127C & 0.9049082 & 7.996086 & $4.00 \mathrm{E}-04$ & 0.04954053 & RSF2 & Zinc-finger protein involved in transcriptional control \\
\hline YOR304C-A & 0.9281514 & 7.981128 & $2.48 \mathrm{E}-04$ & 0.040821618 & BIL1 & Protein that binds Bud6p, role in actin cable assembly \\
\hline YDR078C & 0.9837623 & 7.645733 & 3.24E-04 & 0.045973049 & SHU2 & Component of the SHU complex, functions in promoting error-free DNA repair \\
\hline YLR241W & 0.9923575 & 7.711077 & 3.67E-04 & 0.048372791 & CSC1 & Calcium permeable gated cation channel \\
\hline YLR262C-A & 1.0079829 & 7.993725 & $3.26 \mathrm{E}-04$ & 0.045973049 & TMA7 & Protein of unknown function, associates with ribosomes \\
\hline YKR037C & 1.046689 & 7.997847 & $1.12 \mathrm{E}-04$ & 0.025605971 & SPC34 & Essential subunit of the Dam1 complex \\
\hline YKR019C & 1.0715253 & 8.323317 & $1.32 \mathrm{E}-07$ & 0.000783566 & IRS4 & Protein involved in regulating phosphatidylinositol 4,5-biphosphate levels \\
\hline YNL138W & 1.0798731 & 7.540887 & $1.81 \mathrm{E}-04$ & 0.035772533 & SRV2 & Cyclase-associated protein \\
\hline YGR116W & 1.1179655 & 8.407091 & $4.41 \mathrm{E}-05$ & 0.014538754 & SPT6 & Nucleosome remodelling protein \\
\hline YPL070W & 1.1331816 & 7.868743 & $4.42 \mathrm{E}-05$ & 0.014538754 & MUK1 & Guanine nucleotide exchange factor, involved in transport and trafficking \\
\hline YOL052C & 1.151685 & 6.55401 & 3.63E-04 & 0.048372791 & SPE2 & S-adenosylmethioine decarboxylase, biosynthesis of spermidine and spermine \\
\hline YNL126W & 1.1862742 & 7.383182 & 9.37E-06 & 0.009255003 & SPC98 & Component of the microtubule-nucleating gamma-tubulin complex \\
\hline YIL093C & 1.2049165 & 7.832839 & 2.81E-06 & 0.003462807 & RSM25 & Mitochondrial ribosomal protein of the small subuni \\
\hline YPR133C & 1.2066575 & 7.915618 & 3.87E-04 & 0.04954053 & SPN1 & Protein involved in RNA polymerase II transcription \\
\hline YGL008C & 1.2176259 & 7.3241 & $1.62 \mathrm{E}-05$ & 0.009466682 & PMA1 & Plasma membrane P2-type H+-ATPase, pumps protons out of the cell \\
\hline YOR036W & 1.2323278 & 7.533333 & $2.10 \mathrm{E}-05$ & 0.009466682 & PEP12 & Target membrane receptor, t-SNARE, transport and trafficking \\
\hline YBR102C & 1.2350682 & 6.803939 & 7.12E-05 & 0.018885621 & EX084 & Exocyst subunit with dual roles in exocytosis and spliceosome assembly \\
\hline YBR265W & 1.2465347 & 6.964784 & $3.52 \mathrm{E}-04$ & 0.048372791 & TSC10 & 3-ketospinganine reductase \\
\hline YMR072W & 1.3562235 & 6.439788 & $2.24 \mathrm{E}-05$ & 0.009466682 & ABF2 & Mitochondrial DNA-binding protein \\
\hline YOL019W & 1.3691733 & 7.306941 & $2.70 \mathrm{E}-04$ & 0.042688993 & YOL019W & Protein of unknown function \\
\hline YFR010W & 1.3863256 & 6.847775 & 7.33E-05 & 0.018885621 & UBP6 & Ubiquitin-specific protease \\
\hline YIL141W & 1.4026674 & 8.192527 & 8.90E-07 & 0.001757784 & YIL141W & Dubious ORF \\
\hline YDR054C & 1.4332071 & 6.6478 & $1.64 \mathrm{E}-05$ & 0.009466682 & CDC34 & Ubiquitin-conjugation enzyme \\
\hline YGL232W & 1.5966002 & 6.65792 & $3.79 \mathrm{E}-05$ & 0.01405686 & TAN1 & Putative tRNA acetyletransferase \\
\hline YNL177C & 1.6220951 & 6.186091 & 2.23E-05 & 0.009466682 & MRPL22 & Mitochondrial ribosomal protein of the large subunit \\
\hline YNL062C & 1.6388785 & 8.168126 & $2.92 \mathrm{E}-06$ & 0.003462807 & GCD10 & Subunit of tRNA (1-methyladenosine) methyltransferase with Gcd14p \\
\hline
\end{tabular}


To ascertain the legitimacy of the mutants identified with negative $\log F C<-0.75$ and FDR $<$ 0.05, the 20 heterozygous mutants mentioned above (Table 4.5) were grown in the presence and absence of the $P$. insularum methanolic extract $(0.0002 \% \mathrm{v} / \mathrm{v})$. Seven mutants were shown to be significantly hypersensitive compared to the WT BY4743 control in the presence of the extract (Fig 4.6). These strains include RPL10 involved in ribosomal biogenesis (Planta \& Mager, 1998), SPT16 involved in FACT(facilitator of chromatin transactions) complex (Malone et al., 1991), FLO11 involved in flocculation (Lo \& Dranginis, 1996), SET5 involved in methylation of histones (Nislow et al., 1997), CDC39 involved in the CCR4-NOT complex, which is a complex involved in mRNA metabolism, including the repression and activation of mRNA initiation (Breter et al., 1983), and ATG15 involved in the lysis of autophagic and Cvt (cytoplasm-to-vacuole) transport bodies (Klonsky et al., 2003). The growth of the dubious ORF YKLO36C was also shown to be significantly impaired in the presence of the methanolic extract of $P$. insularum.

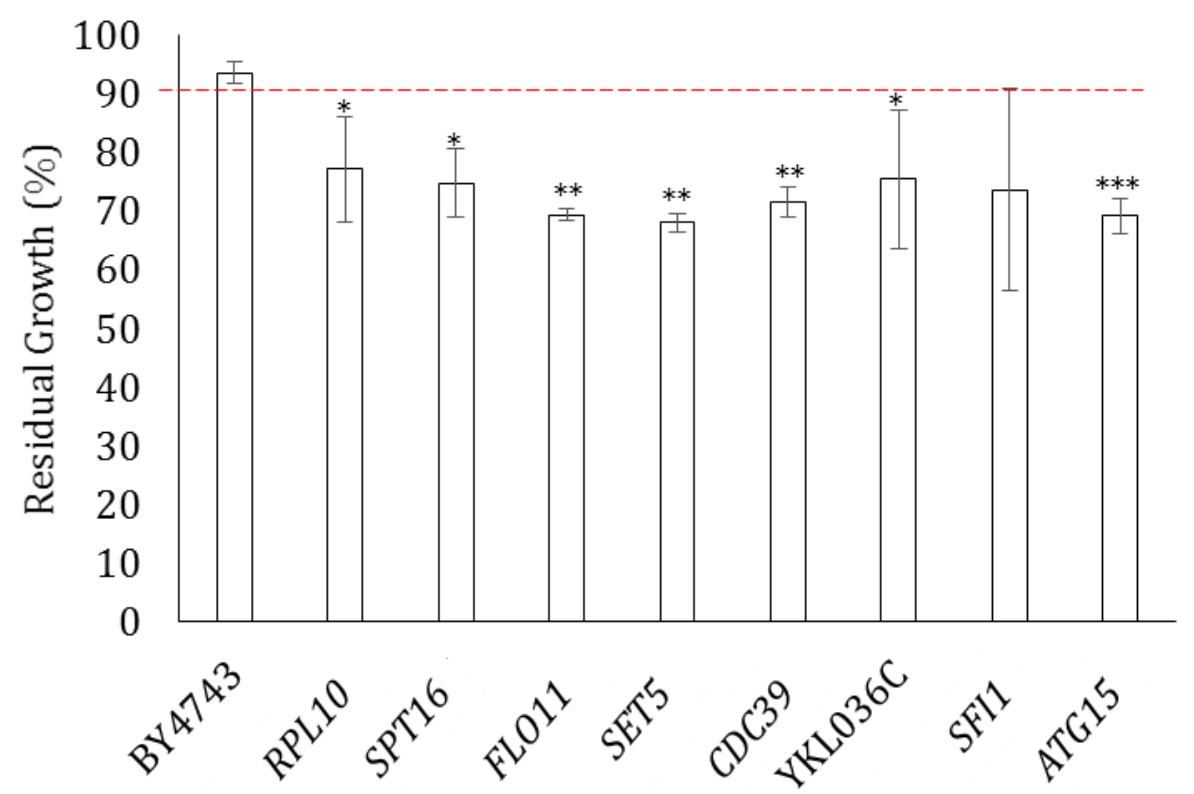

Figure 4.6: Validations of the heterozygote genes detected to be hypersensitive to the methanolic extract of $\boldsymbol{P}$. insularum. Heterozygous mutants were individually grown in the presence of the methanolic extract in triplicate, and compared to the control treatment. Mutants exhibiting growth less than $90 \%$ consistently across the three triplicates are plotted compared to WT (BY4743), are reported as mean residual growth $\pm \mathrm{SD}$. The p-values were calculated using a one tailed Student's t-test $\left(*\right.$ : p-value $<0.05,{ }^{* *}$ : p-value $<0.01,{ }^{* * *}$ : p-value $\left.<0.001\right)$. 


\subsubsection{HOP Analysis of the P. insularum Methanolic Extract}

To identify the genes buffering the bioactivity of the methanolic extract from $P$. insularum leaves, a HOP analysis was conducted using Bar-seq analysis. The pooled homozygous library was grown in the presence and absence of $0.0001 \% \mathrm{v} / \mathrm{v}$ of the methanolic extract for 20 generations. Using FDR < 0.05 as a cutoff for statistical significance, there were 106 deletion mutants with positive logFC ranging from 0.5 to 4.6, and 182 deletion mutants with negative logFC ranging from0.5 to -5.3 , suggesting these homozygous deletion strains grew significantly different from that of WT as a result of the methanolic extract treatment (Fig 4.7). The recovery of 288 total mutants sensitive in the HOP analysis of the methanolic $P$. insularum extract was markedly greater than the 30 mutants that were sensitive in the HOP analysis of the aqueous $P$. insularum extract. Analysis of this list of 306 genes for GO enrichment found 4 biological processes were enriched, including the negative regulation of transcription from RNA polymerase II promoter (21 genes, p-value 0.006881), cytoplasmic translation (23 genes, p-value 0.020136), histone deacetylation ( 8 genes, 0.023809) and protein deacetylation (8 genes, p-value 0.046855). One molecular function was enriched, which involved structural constituent of ribosome (26 genes, p-value 0.010379). Eight molecular components were enriched, including cytosolic large ribosomal subunit (18 genes, p-value $1.084534^{-}$ ${ }^{4}$ ), cytosolic ribosome (24 genes, p-value $2.613199^{-4}$ ), Set3 complex (5 genes, p-value 0.003051), large ribosome subunit (20 genes, $p$-value 0.003838$)$, ribosomal subunit (27 genes, p-value $0.006386)$ and cytosolic part (26 genes, p-value 0.014001).

The 182 gene deletions with reduced growth due to the $P$. insularum methanolic extract are involved in a variety of functions (Table 4.7), including cytoplasmic translation, ribosomal biogenesis, and transcriptional regulation (NOT3, NAP1, HDA2, HDA3, RRM3 and SWP82) (Cairns et al., 1994; Collart \& Struhl, 1994; Ishimi \& Kikuchi, 1991; Ivessa et al., 2000; Wu et al., 2000; Inadome et al., 2005; Muren et al., 2001; Piper et al., 1995; Rothman et al., 1989; Wichmann et al., 1992; Yompakdee et al., 1996). Several dubious ORFs and proteins of unknown functions were also detected to be hypersensitive, including YLR407W, RIM9, YPL261C, YCR050C, YCR049C, FYV1, 
YERO84W and YDR417C. Interestingly, both YCRO49C and YCRO50C detected as hypersensitive hits overlap ARE1, which encodes acyl-CoA:sterolacyltransferase, an ER enzyme involved in sterol esterification (Yang et al., 1996; Yu et al., 1996).

The 106 genes corresponding to the deletion mutants with improved growth in the presence of the extract (Table 4.8) are involved in a variety of biological processes, including DNAtemplated regulation of transcription (31 genes, e.g. SIF2, MRC1, SNT1, GIS1, HTA1, YAP6, SPT2, SPT3, MIG3, LRS4, IES1 and PGD1), regulation of cellular macromolecule biosynthetic pathway (32 genes) and the regulation of nitrogen compound metabolic process involving 33 genes. As these processes are related, genes were detected from all three enriched biological processes, such as SIF2, SPT2 and SPT3.

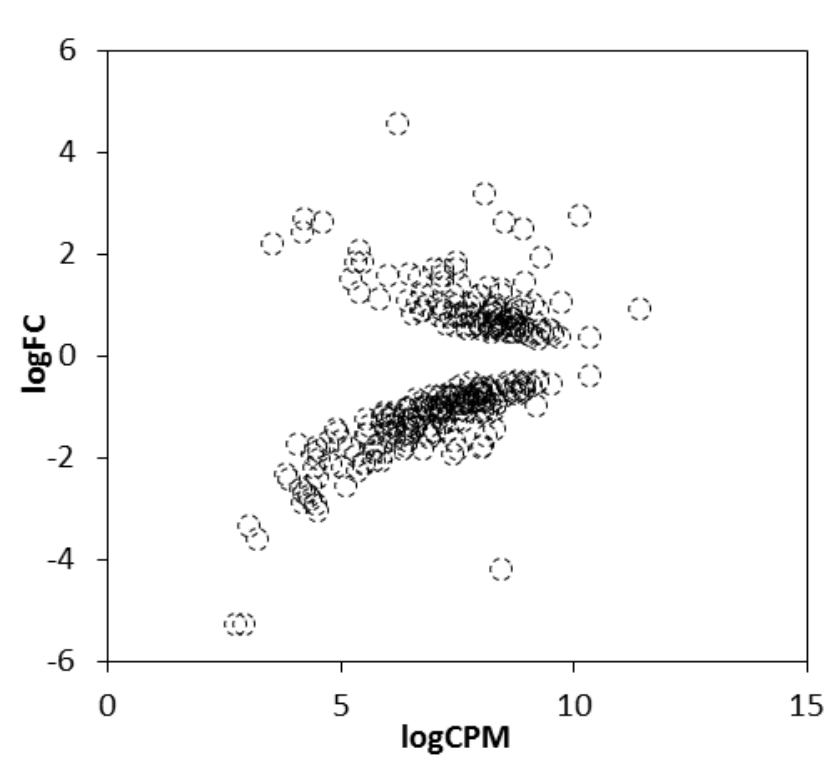

Figure 4.7: Scatterplot of 288 hypersensitive homozygous mutants against the methanolic extract of $\boldsymbol{P}$. insularum. Pooled homozygous deletion mutants were grown in the presence of $0.0001 \%$ $\mathrm{v} / \mathrm{v}$ of methanolic P. insularum extract over 20 generations, before DNA extraction and barcode sequenced. Mutants with calculated FDR values less than 0.05 were plotted, using $\log$ CPM vs $\log F C$. 
Table 4.7: $\quad$ Hypersensitive homozygous deletions against treatment with the methanol extract of $P$. insularum. The data summarized herein are sorted by the $\log \mathrm{FC}$, with the lowest logFC at the top of the table, indicating the genes are sorted from most hypersensitive to the least hypersensitive true hits. logCPM, pvalue and FDR values for all gene hits are shown, together with their gene names and biological functions obtained from the Saccharomyces Gene Database.

\begin{tabular}{|c|c|c|c|c|c|c|}
\hline & $\log \mathrm{FC}$ & $\log \mathrm{CPM}$ & PValue & FDR & Gene & Biological Function \\
\hline YLR131C & -4.17683 & 8.402519 & $9.20 \mathrm{E}-05$ & 4.83E-03 & ACE2 & Transcription factor required for septum destruction after cytokinesis \\
\hline YFL025C & -3.57606 & 3.189629 & $1.08 \mathrm{E}-04$ & $5.26 \mathrm{E}-03$ & BST1 & GPI inositol deacylase of the endoplasmic reticulum (ER) \\
\hline YDL047W & -3.336 & 3.010566 & $2.85 \mathrm{E}-04$ & $1.08 \mathrm{E}-02$ & SIT4 & Type 2A-related serine-threonine phosphatase \\
\hline YDL081C & -3.0367 & 4.471486 & $3.21 \mathrm{E}-05$ & $2.29 \mathrm{E}-03$ & RPP1A & Ribosomal stalk protein P1 alpha \\
\hline YML001W & -2.88744 & 4.426691 & $1.12 \mathrm{E}-06$ & $1.64 \mathrm{E}-04$ & YPT7 & Rab family GTPase \\
\hline YML073C & -2.88005 & 4.1334 & $6.02 \mathrm{E}-05$ & $3.43 \mathrm{E}-03$ & RPL6A & Ribosomal $60 \mathrm{~S}$ subunit protein L6A \\
\hline YOL052C & -2.79799 & 4.312444 & 1.07E-03 & $2.71 \mathrm{E}-02$ & SPE2 & S-adenosylmethionine decarboxylase; required for the biosynthesis of spermidine and spermine \\
\hline YJL121C & -2.74276 & 4.292456 & $1.03 \mathrm{E}-03$ & 2.63E-02 & RPE1 & D-ribulose-5-phosphate 3-epimerase \\
\hline YCL058C & -2.66391 & 4.177405 & $1.85 \mathrm{E}-05$ & $1.46 \mathrm{E}-03$ & FYV5 & Protein involved in regulation of the mating pathway \\
\hline YNL055C & -2.59417 & 4.104447 & $6.75 \mathrm{E}-04$ & $1.95 \mathrm{E}-02$ & POR1 & Mitochondrial porin (voltage-dependent anion channel) \\
\hline YKL184W & -2.54098 & 4.340093 & $1.33 \mathrm{E}-03$ & $3.15 \mathrm{E}-02$ & SPE1 & Ornithine decarboxylase; catalyzes the first step in polyamine biosynthesis \\
\hline YLL043W & -2.53832 & 5.065306 & $3.81 \mathrm{E}-05$ & $2.54 \mathrm{E}-03$ & FPS1 & Aquaglyceroporin, plasma membrane channel \\
\hline YBL027W & -2.40995 & 3.853045 & $2.00 \mathrm{E}-05$ & $1.56 \mathrm{E}-03$ & RPL19B & Ribosomal 60S subunit protein L19B \\
\hline YNR006W & -2.38934 & 4.473799 & $8.20 \mathrm{E}-05$ & 4.38E-03 & VPS27 & Endosomal protein that forms a complex with Hse1p \\
\hline YJR074W & -2.29337 & 3.781547 & 2.77E-04 & $1.06 \mathrm{E}-02$ & MOG1 & Conserved nuclear protein that interacts with GTP-Gsp1p \\
\hline YBR106W & -2.2413 & 5.333605 & $6.13 \mathrm{E}-04$ & $1.82 \mathrm{E}-02$ & PHO88 & Probable membrane protein; involved in phosphate transport, secretory protein maturation \\
\hline YOR070C & -2.1744 & 5.012808 & $1.52 \mathrm{E}-03$ & $3.48 \mathrm{E}-02$ & GYP1 & Cis-golgi GTPase-activating protein (GAP) for yeast Rabs \\
\hline YMR269W & -2.16336 & 4.381987 & $2.18 \mathrm{E}-05$ & $1.62 \mathrm{E}-03$ & TMA23 & Nucleolar protein implicated in ribosome biogenesis \\
\hline YJL204C & -2.1117 & 5.473082 & $4.81 \mathrm{E}-06$ & $4.68 \mathrm{E}-04$ & RCY1 & F-box protein involved in recycling endocytosed proteins \\
\hline YGL124C & -2.09976 & 4.765319 & 3.48E-05 & 2.37E-03 & MON1 & Subunit of a heterodimeric guanine nucleotide exchange factor (GEF) \\
\hline YDR435C & -2.08545 & 5.45808 & $6.05 \mathrm{E}-06$ & $5.78 \mathrm{E}-04$ & PPM1 & Carboxyl methyltransferase \\
\hline YIL038C & -2.06305 & 5.678219 & $2.65 \mathrm{E}-06$ & $2.90 \mathrm{E}-04$ & NOT3 & Subunit of CCR4-NOT global transcriptional regulator \\
\hline YEL054C & -2.04165 & 5.827001 & $2.66 \mathrm{E}-04$ & $1.02 \mathrm{E}-02$ & RPL12A & Ribosomal 60S subunit protein L12A \\
\hline YNR051C & -2.02342 & 4.5108 & $2.30 \mathrm{E}-03$ & $4.71 \mathrm{E}-02$ & BRE5 & Ubiquitin protease cofactor \\
\hline YAL053W & -1.95589 & 5.273208 & $2.38 \mathrm{E}-05$ & $1.74 \mathrm{E}-03$ & FLC2 & Putative calcium channel involved in calcium release under hypotonic stress \\
\hline YOR275C & -1.9199 & 7.373076 & $1.54 \mathrm{E}-09$ & $5.38 \mathrm{E}-07$ & RIM20 & Protein involved in proteolytic activation of Rim101p \\
\hline YPL065W & -1.90951 & 4.342689 & $1.04 \mathrm{E}-03$ & 2.65E-02 & VPS28 & Component of the ESCRT-I complex \\
\hline YKL149C & -1.88056 & 5.683507 & $1.27 \mathrm{E}-06$ & $1.80 \mathrm{E}-04$ & DBR1 & RNA lariat debranching enzyme \\
\hline YLR407W & -1.87084 & 5.886563 & $1.95 \mathrm{E}-04$ & $7.99 \mathrm{E}-03$ & YLR407W & Putative protein of unknown function \\
\hline YMR063W & -1.85593 & 4.48586 & $4.50 \mathrm{E}-04$ & $1.48 \mathrm{E}-02$ & RIM9 & Plasma membrane protein of unknown function \\
\hline
\end{tabular}




\begin{tabular}{|c|c|c|c|c|c|c|}
\hline & $\log \mathrm{FC}$ & $\log \mathrm{CPM}$ & PValue & FDR & Gene & Biological Function \\
\hline YKL176C & -1.82436 & 5.671488 & $2.19 \mathrm{E}-06$ & $2.64 \mathrm{E}-04$ & LST4 & Subunit of the Lst4p-Lst7p GTPase activating protein complex for Gtr2p \\
\hline YPL261C & -1.82045 & 6.729207 & 3.79E-09 & $1.12 \mathrm{E}-06$ & YPL261C & Dubious open reading frame \\
\hline YIL110W & -1.81577 & 7.487026 & $5.88 \mathrm{E}-05$ & $3.39 \mathrm{E}-03$ & HPM1 & AdoMet-dependent methyltransferase \\
\hline YKR007W & -1.80334 & 6.279483 & $1.66 \mathrm{E}-07$ & $3.51 \mathrm{E}-05$ & MEH1 & Component of the EGO and GSE complexes \\
\hline YCR050C & -1.80029 & 4.444633 & 7.75E-04 & 2.17E-02 & YCR050C & Non-essential protein of unknown function \\
\hline YOR043W & -1.78456 & 7.960328 & $4.74 \mathrm{E}-04$ & $1.53 \mathrm{E}-02$ & WHI2 & Protein required for full activation of the general stress response \\
\hline YIL148W & -1.77135 & 5.131166 & $1.56 \mathrm{E}-04$ & $6.68 \mathrm{E}-03$ & RPL40A & Ubiquitin-ribosomal 60S subunit protein L40A fusion protein \\
\hline YPL170W & -1.762 & 8.022655 & $2.02 \mathrm{E}-10$ & $9.22 \mathrm{E}-08$ & DAP1 & Heme-binding protein; involved in regulation of cytochrome P450 protein Erg11p \\
\hline YKR048C & -1.74429 & 6.358735 & $4.28 \mathrm{E}-04$ & $1.42 \mathrm{E}-02$ & NAP1 & Histone chaperone \\
\hline YML017W & -1.73984 & 5.710861 & $3.81 \mathrm{E}-04$ & $1.31 \mathrm{E}-02$ & PSP2 & Asn rich cytoplasmic protein that contains RGG motifs, role in mitochondria mRNA splicing \\
\hline YDR195W & -1.71316 & 4.04087 & 2.33E-03 & $4.72 \mathrm{E}-02$ & REF2 & RNA-binding protein; involved in the cleavage step of mRNA 3 '-end \\
\hline YAR014C & -1.68019 & 4.691206 & $2.55 \mathrm{E}-03$ & $4.98 \mathrm{E}-02$ & BUD14 & Protein involved in bud-site selection \\
\hline YOR030W & -1.66988 & 5.887894 & $2.60 \mathrm{E}-04$ & $1.01 \mathrm{E}-02$ & DFG16 & Probable multiple transmembrane protein, possible involvement with Rim proteins \\
\hline YDR389W & -1.65668 & 4.984338 & $1.84 \mathrm{E}-04$ & $7.59 \mathrm{E}-03$ & SAC7 & GTPase activating protein (GAP) for Rho1p \\
\hline YFR008W & -1.6454 & 6.388552 & $2.44 \mathrm{E}-06$ & 2.83E-04 & FAR7 & Protein involved in recovery from pheromone-induced cell cycle arrest \\
\hline YPR179C & -1.64492 & 7.404422 & 3.07E-08 & $7.28 \mathrm{E}-06$ & HDA3 & Subunit of the HDA1 histone deacetylase complex \\
\hline YDR025W & -1.63371 & 6.100309 & $6.03 \mathrm{E}-07$ & $9.93 \mathrm{E}-05$ & RPS11A & Protein component of the small (40S) ribosomal subuni \\
\hline YBR296C & -1.5986 & 6.867357 & $1.62 \mathrm{E}-06$ & $2.09 \mathrm{E}-04$ & PHO89 & Plasma membrane $\mathrm{Na}+$ /Pi cotransporter \\
\hline YKR027W & -1.56471 & 8.102546 & $2.01 \mathrm{E}-16$ & $1.32 \mathrm{E}-13$ & $\mathrm{BCH} 2$ & Member of the ChAPs (Chs5p-Arf1p-binding proteins) family \\
\hline YHL007C & -1.54929 & 6.260396 & $1.11 \mathrm{E}-05$ & $9.39 \mathrm{E}-04$ & STE20 & Cdc42p-activated signal transducing kinase \\
\hline YDR500C & -1.53462 & 5.55685 & $1.23 \mathrm{E}-03$ & $3.00 \mathrm{E}-02$ & RPL37B & Ribosomal 60S subunit protein L37B \\
\hline YGL031C & -1.52388 & 6.817099 & $5.54 \mathrm{E}-07$ & 9.39E-05 & RPL24A & Ribosomal 60S subunit protein L24A \\
\hline YDL130W & -1.51924 & 6.441736 & $5.34 \mathrm{E}-05$ & $3.13 \mathrm{E}-03$ & RPP1B & Ribosomal protein P1 beta \\
\hline YNL294C & -1.51679 & 6.352535 & $6.13 \mathrm{E}-05$ & $3.43 \mathrm{E}-03$ & RIM21 & pH sensor molecule, component of the RIM101 pathway \\
\hline YHR031C & -1.50966 & 5.511805 & $2.20 \mathrm{E}-04$ & $8.75 \mathrm{E}-03$ & RRM3 & DNA helicase involved in rDNA replication and Ty1 transposition \\
\hline YCR049C & -1.50055 & 6.98131 & $2.53 \mathrm{E}-07$ & $4.84 \mathrm{E}-05$ & YCR049C & Dubious open reading frame \\
\hline YDR024W & -1.49943 & 6.121972 & $1.06 \mathrm{E}-04$ & $5.24 \mathrm{E}-03$ & FYV1 & Dubious open reading frame \\
\hline YNL323W & -1.4747 & 4.880125 & $1.99 \mathrm{E}-03$ & $4.25 \mathrm{E}-02$ & LEM3 & Membrane protein of the plasma membrane and ER \\
\hline YDR295C & -1.47261 & 7.262973 & $6.50 \mathrm{E}-07$ & $1.04 \mathrm{E}-04$ & HDA2 & Subunit of the HDA1 histone deacetylase complex \\
\hline YER177W & -1.47016 & 6.978217 & $9.66 \mathrm{E}-04$ & $2.50 \mathrm{E}-02$ & BMH1 & 14-3-3 protein, major isoform \\
\hline
\end{tabular}




\begin{tabular}{|c|c|c|c|c|c|c|}
\hline & $\log \mathrm{FC}$ & $\log \mathrm{CPM}$ & PValue & FDR & Gene & Biological Function \\
\hline YER084W & -1.45349 & 6.405747 & $6.31 \mathrm{E}-06$ & $5.94 \mathrm{E}-04$ & YER084W & Protein of unknown function \\
\hline YKR035W-A & -1.42932 & 4.860043 & $1.35 \mathrm{E}-03$ & 3.17E-02 & DID2 & Class E protein of the vacuolar protein-sorting (Vps) pathway \\
\hline YKL190W & -1.39118 & 6.582962 & 1.04E-04 & $5.23 \mathrm{E}-03$ & CNB1 & Calcineurin B; regulatory subunit of calcineurin which regulates Crz1p \\
\hline YGL045W & -1.37923 & 6.536361 & $1.08 \mathrm{E}-04$ & $5.26 \mathrm{E}-03$ & RIM8 & Protein involved in proteolytic activation of Rim101p \\
\hline YDR417C & -1.36016 & 6.395482 & $4.43 \mathrm{E}-05$ & $2.79 \mathrm{E}-03$ & YDR417C & Dubious open reading frame \\
\hline YOR246C & -1.34796 & 6.075999 & $5.75 \mathrm{E}-04$ & $1.72 \mathrm{E}-02$ & ENV9 & Protein proposed to be involved in vacuolar functions \\
\hline YLR185W & -1.34586 & 5.587265 & 4.15E-04 & 1.39E-02 & RPL37A & Ribosomal 60S subunit protein L37A \\
\hline YMR238W & -1.34429 & 6.092986 & $1.84 \mathrm{E}-04$ & $7.59 \mathrm{E}-03$ & DFG5 & Putative mannosidase \\
\hline YGL253W & -1.34219 & 6.855512 & 1.79E-04 & 7.47E-03 & HXK2 & Hexokinase isoenzyme 2 \\
\hline YLR047C & -1.32811 & 5.868224 & $1.23 \mathrm{E}-03$ & $3.00 \mathrm{E}-02$ & FRE8 & Protein with sequence similarity to iron/copper reductases; involved in iron homeostasis \\
\hline YHR203C & -1.32 & 6.116239 & 7.89E-04 & 2.19E-02 & RPS4B & Protein component of the small (40S) ribosomal subunit \\
\hline YMR052C-A & -1.29154 & 7.172126 & $2.43 \mathrm{E}-06$ & 2.83E-04 & YMR052C-A & Dubious open reading frame \\
\hline YAR042W & -1.29037 & 7.200968 & 4.12E-04 & 1.39E-02 & SWH1 & Protein similar to mammalian oxysterol-binding protein \\
\hline YHR200W & -1.27628 & 6.365135 & $1.51 \mathrm{E}-04$ & $6.62 \mathrm{E}-03$ & RPN10 & Non-ATPase base subunit of the 19S RP of the 26S proteasome \\
\hline YNL032W & -1.26573 & 7.451078 & 4.57E-07 & 8.47E-05 & SIW14 & Tyrosine phosphatase involved in actin organization and endocytosis \\
\hline YMR071C & -1.2503 & 7.572523 & 4.43E-05 & 2.79E-03 & TVP18 & Integral membrane protein; localized to late Golgi vesicles along with the v-SNARE Tlg2p \\
\hline YCR087W & -1.24338 & 6.654061 & 1.73E-04 & 7.28E-03 & YCR087W & Dubious open reading frame \\
\hline YGL005C & -1.24176 & 5.510767 & $2.35 \mathrm{E}-03$ & $4.74 \mathrm{E}-02$ & COG7 & Component of the conserved oligomeric Golgi complex \\
\hline YGR133W & -1.24127 & 6.420837 & 8.45E-04 & $2.28 \mathrm{E}-02$ & PEX4 & Peroxisomal ubiquitin conjugating enzyme \\
\hline YHR066W & -1.24042 & 5.988224 & $1.96 \mathrm{E}-03$ & 4.22E-02 & SSF1 & Constituent of $66 \mathrm{~S}$ pre-ribosomal particles \\
\hline YGL046W & -1.2401 & 6.4959 & 1.97E-03 & 4.22E-02 & YGL046W & Merged ORF, does not encode a protein, overlaps with RIM8 \\
\hline YGL035C & -1.23062 & 7.198744 & $9.40 \mathrm{E}-07$ & 1.47E-04 & MIG1 & Transcription factor involved in glucose repression \\
\hline YGR054W & -1.20427 & 7.077133 & $1.41 \mathrm{E}-06$ & $1.94 \mathrm{E}-04$ & YGR054W & Eukaryotic initiation factor (eIF) $2 \mathrm{~A}$ \\
\hline YBR277C & -1.19526 & 6.067662 & $1.15 \mathrm{E}-03$ & 2.88E-02 & YBR277C & Dubious open reading frame \\
\hline YFL049W & -1.19309 & 6.652561 & $1.22 \mathrm{E}-04$ & $5.59 \mathrm{E}-03$ & SWP82 & Member of the SWI/SNF chromatin remodeling complex \\
\hline YBR077C & -1.1619 & 5.891383 & $9.79 \mathrm{E}-04$ & $2.51 \mathrm{E}-02$ & SLM4 & Component of the EGO and GSE complexes, microautophagy and amino acid sorting \\
\hline YEL053C & -1.15791 & 5.880754 & $2.30 \mathrm{E}-03$ & $4.71 \mathrm{E}-02$ & MAK10 & Non-catalytic subunit of N-terminal acetyltransferase of the NatC type \\
\hline YBR300C & -1.12914 & 8.095591 & 4.36E-06 & 4.31E-04 & YBR300C & Dubious open reading frame \\
\hline YIL133C & -1.11967 & 6.087205 & $5.20 \mathrm{E}-04$ & $1.64 \mathrm{E}-02$ & RPL16A & Ribosomal 60S subunit protein L16A \\
\hline YNR032W & -1.10894 & 8.115949 & $6.60 \mathrm{E}-09$ & $1.78 \mathrm{E}-06$ & PPG1 & Putative serine/threonine protein phosphatase \\
\hline
\end{tabular}


Table 4.8: $\quad$ Top 90 gene deletions exhibiting improved growth in the presence of the methanolic extract of $P$. insularum. The data summarized herein are sorted by the $\operatorname{logFC}$, with the lowest $\operatorname{logFC}$ at the top of the table, indicating the genes are sorted from most hypersensitive to the least hypersensitive true hits. $\log \mathrm{CPM}, \mathrm{p}$-value and FDR values for all gene hits are shown, together with their gene names and biological functions obtained from the Saccharomyces Gene Database.

\begin{tabular}{|c|c|c|c|c|c|c|}
\hline & $\log \mathrm{FC}$ & $\log \mathrm{CPM}$ & PValue & FDR & Gene & Biological Function \\
\hline YDR392W & 4.5919828 & 6.188196 & $8.80 \mathrm{E}-17$ & $7.00 \mathrm{E}-14$ & SPT3 & Subunit of the SAGA and SAGA-like transcriptional regulatory complex \\
\hline YGL255W & 3.2012021 & 8.034208 & $1.72 \mathrm{E}-04$ & $7.28 \mathrm{E}-03$ & ZRT1 & High-affinity zinc transporter of the plasma membrane \\
\hline YPR065W & 2.7891216 & 10.089961 & $2.01 \mathrm{E}-36$ & $1.19 \mathrm{E}-32$ & ROX1 & Heme-dependent repressor of hypoxic genes \\
\hline YDR401W & 2.718588 & 4.179569 & 2.33E-04 & $9.21 \mathrm{E}-03$ & YDR401W & Dubious ORF \\
\hline YLR055C & 2.6536338 & 8.470276 & $1.29 \mathrm{E}-21$ & $2.55 \mathrm{E}-18$ & SPT8 & Subunit of the SAGA transcriptional regulatory complex \\
\hline YGL025C & 2.6302753 & 4.555716 & $7.39 \mathrm{E}-04$ & $2.10 \mathrm{E}-02$ & PGD1 & Subunit of the RNA polymerase II mediator complex \\
\hline YML007W & 2.5193522 & 8.876484 & 8.08E-20 & $1.20 \mathrm{E}-16$ & YAP1 & Transcriptional factor required for oxidative stress tolerance \\
\hline YNL236W & 2.441835 & 4.138878 & $1.00 \mathrm{E}-06$ & $1.52 \mathrm{E}-04$ & SIN4 & Subunit of the RNA polymerase II mediator complex \\
\hline YNL107W & 2.2009873 & 3.504036 & $2.51 \mathrm{E}-03$ & 4.94E-02 & YAF9 & Subunit of the NuA4 histone $\mathrm{H} 4$ acetyltransferase and SWR1 complexes \\
\hline YML010C-B & 2.0865442 & 5.373827 & $1.53 \mathrm{E}-04$ & $6.64 \mathrm{E}-03$ & YML010C-B & Dubious ORF \\
\hline YPR064W & 1.9660034 & 9.279071 & $1.05 \mathrm{E}-19$ & $1.25 \mathrm{E}-16$ & YPR064W & Dubious ORF \\
\hline YDL002C & 1.8663376 & 7.433041 & $9.44 \mathrm{E}-17$ & $7.00 \mathrm{E}-14$ & NHP10 & Non-essential IN080 chromatin remodeling complex subunit \\
\hline YBL047C & 1.8554673 & 5.274969 & 3.37E-04 & $1.22 \mathrm{E}-02$ & EDE1 & Scaffold protein involved in the formation of early endocytic sites \\
\hline YNL079C & 1.8413202 & 5.446698 & $1.23 \mathrm{E}-04$ & $5.59 \mathrm{E}-03$ & TPM1 & Major isoform of tropomyosin, stabilizes cables and filaments \\
\hline YGR102C & 1.7393452 & 7.425531 & $3.67 \mathrm{E}-13$ & $2.18 \mathrm{E}-10$ & GTF1 & Subunit of the trimeric GatFAB Amido Transferase (AdT) complex \\
\hline YBL065W & 1.73587 & 7.144636 & $4.68 \mathrm{E}-04$ & $1.53 \mathrm{E}-02$ & YBL065W & Dubious ORF \\
\hline YDL082W & 1.7347822 & 6.987405 & $1.97 \mathrm{E}-03$ & $4.22 \mathrm{E}-02$ & RPL13A & Ribosomal 60S subunit protein L13A \\
\hline YDR268W & 1.6410044 & 6.431576 & $2.37 \mathrm{E}-07$ & 4.69E-05 & MSW1 & Mitochondrial tryptophanyl-tRNA synthetase \\
\hline YKL032C & 1.5996274 & 7.160644 & $2.60 \mathrm{E}-09$ & $8.55 \mathrm{E}-07$ & IXR1 & Transcriptional repressor that regulates hypoxic genes during normoxia \\
\hline YML094W & 1.5928865 & 5.975458 & $2.57 \mathrm{E}-03$ & $4.98 E-02$ & GIM5 & Subunit of the heterohexameric cochaperone prefoldin complex \\
\hline YDR439W & 1.5765991 & 6.641002 & 3.09E-06 & $3.21 \mathrm{E}-04$ & LRS4 & Nucleolar protein that forms complex with Csm1p \\
\hline YLR233C & 1.5382738 & 5.194209 & $5.75 \mathrm{E}-04$ & $1.72 \mathrm{E}-02$ & EST1 & TLC1 RNA-associated factor involved in telomere length regulation \\
\hline YLL013C & 1.4729478 & 8.923422 & $4.06 \mathrm{E}-18$ & $4.01 \mathrm{E}-15$ & PUF3 & Protein of the mitochondrial outer surface \\
\hline YEL050C & 1.4576679 & 7.15631 & $8.10 \mathrm{E}-04$ & $2.22 \mathrm{E}-02$ & RML2 & Mitochondrial ribosomal protein of the large subunit (L2) \\
\hline YDL146W & 1.4153258 & 7.523979 & 5.19E-05 & $3.11 \mathrm{E}-03$ & LDB17 & Protein involved in the regulation of endocytosis \\
\hline YBR216C & 1.3548683 & 8.137438 & 3.07E-09 & $9.59 \mathrm{E}-07$ & YBP1 & Protein involved in cellular response to oxidative stress \\
\hline YCR033W & 1.3379052 & 8.412615 & $1.39 \mathrm{E}-12$ & $7.50 \mathrm{E}-10$ & SNT1 & Subunit of the Set3C deacetylase complex \\
\hline YOR002W & 1.2553948 & 5.371411 & 4.89E-04 & $1.57 \mathrm{E}-02$ & ALG6 & Alpha 1,3 gluosyltransferase \\
\hline YER028C & 1.2424489 & 8.419123 & $2.92 \mathrm{E}-10$ & $1.24 \mathrm{E}-07$ & MIG3 & Transcriptional regulator \\
\hline YDR314C & 1.2295853 & 6.720442 & 1.59E-03 & $3.62 \mathrm{E}-02$ & RAD34 & Protein involved in nucleotide excision repair (NER) \\
\hline
\end{tabular}


Table 4.8 Cont'd

\begin{tabular}{|c|c|c|c|c|c|c|}
\hline & $\operatorname{logFC}$ & $\log \mathrm{CPM}$ & PValue & FDR & Gene & Biological Function \\
\hline YBR103W & 1.2090631 & 7.931573 & $4.56 \mathrm{E}-10$ & $1.80 \mathrm{E}-07$ & SIF2 & WD40 repeat-containing subunit of Set3C histone deacetylase complex \\
\hline YFL013C & 1.189818 & 7.957044 & 8.59E-08 & $1.89 \mathrm{E}-05$ & IES1 & Subunit of the INO80 chromatin remodeling complex \\
\hline YKL114C & 1.1306098 & 5.80748 & 4.77E-04 & $1.54 \mathrm{E}-02$ & APN1 & Major apurinic/apyriminidinic endonuclease \\
\hline YDR225W & 1.0936336 & 6.400817 & $2.32 \mathrm{E}-03$ & $4.72 \mathrm{E}-02$ & HTA1 & Histone $\mathrm{H} 2 \mathrm{~A}$ \\
\hline YGL043W & 1.0738261 & 6.622582 & $3.79 \mathrm{E}-04$ & $1.31 \mathrm{E}-02$ & DST1 & General transcription elongation factor TFIIS \\
\hline YJL052W & 1.0688412 & 9.686078 & $2.58 \mathrm{E}-08$ & $6.38 \mathrm{E}-06$ & TDH1 & Glyceraldehyde-3-phosphate dehydrogenase (GAPDH) isozyme 1 \\
\hline YKL115C & 1.0546653 & 7.845915 & $8.45 \mathrm{E}-08$ & $1.89 \mathrm{E}-05$ & YKL115C & Dubious ORF \\
\hline YIR037W & 1.0424254 & 9.106641 & $1.41 \mathrm{E}-08$ & $3.62 \mathrm{E}-06$ & HYR1 & Thiol peroxidase \\
\hline YOR251C & 1.0382226 & 7.098727 & $9.24 \mathrm{E}-04$ & $2.43 \mathrm{E}-02$ & TUM1 & Rhodanese domain sulfur transferase \\
\hline YOR189W & 1.0197604 & 8.262246 & $5.00 \mathrm{E}-05$ & $3.05 \mathrm{E}-03$ & IES4 & Component of the INO80 chromatin remodeling complex \\
\hline YMR156C & 1.0159901 & 7.44248 & $3.90 \mathrm{E}-04$ & $1.33 \mathrm{E}-02$ & TPP1 & DNA 3'-phosphatase \\
\hline YKL134C & 1.0111059 & 6.797414 & 1.37E-03 & 3.19E-02 & oCT1 & Mitochondrial intermediate peptidase \\
\hline YFL013W-A & 0.9959433 & 8.886304 & $1.21 \mathrm{E}-10$ & $5.99 \mathrm{E}-08$ & YFL013W-A & Dubious ORF \\
\hline YJL169W & 0.9917292 & 7.714283 & $1.44 \mathrm{E}-06$ & $1.94 \mathrm{E}-04$ & YJL169W & Dubious ORF \\
\hline YER092W & 0.9706077 & 8.25769 & $2.51 \mathrm{E}-06$ & $2.86 \mathrm{E}-04$ & IES5 & Non-essential INO80 chromatin remodeling complex subunit \\
\hline YFL033C & 0.9464532 & 11.37304 & $4.19 \mathrm{E}-25$ & $1.24 \mathrm{E}-21$ & RIM15 & Protein kinase involved in cell proliferation in response to nutrients \\
\hline YGR012W & 0.9434509 & 7.120897 & 4.03E-05 & 2.63E-03 & MCY1 & Putative cysteine synthase \\
\hline YHR009C & 0.9346516 & 8.731417 & $5.17 \mathrm{E}-10$ & $1.92 \mathrm{E}-07$ & TDA3 & Putative oxidoreductase involved in late endosome to Golgi transport \\
\hline YGL028C & 0.9106868 & 6.718832 & $6.42 \mathrm{E}-04$ & $1.88 \mathrm{E}-02$ & SCW11 & Cell wall protein with similarity to glucanases \\
\hline YPL049C & 0.9062566 & 7.204247 & $1.16 \mathrm{E}-03$ & $2.90 \mathrm{E}-02$ & DIG1 & MAP kinase-resonsive inhibitor of the Ste12p transcription factor \\
\hline YGL087C & 0.9054502 & 8.186316 & $2.77 \mathrm{E}-06$ & $2.98 \mathrm{E}-04$ & MMS2 & Ubiquitin-conjugating enzyme variant \\
\hline YCL060C & 0.9043242 & 6.676263 & $1.96 \mathrm{E}-03$ & $4.22 \mathrm{E}-02$ & $<\mathrm{NA}>$ & Merged ORF with MRC1: S-phase checkpoint protein \\
\hline YLR150W & 0.9013719 & 9.211196 & $2.24 \mathrm{E}-07$ & $4.58 \mathrm{E}-05$ & STM1 & Required for optimal translation under nutrient stress \\
\hline YIL035C & 0.8969374 & 8.202944 & $1.53 \mathrm{E}-06$ & $2.02 \mathrm{E}-04$ & CKA1 & Alpha catalytic subunit of casein kinase 2 (CK2) \\
\hline YER161C & 0.8947903 & 8.848305 & 4.83E-07 & 8.68E-05 & SPT2 & Protein involved in negative regulation of transcription \\
\hline YPL182C & 0.8799259 & 7.697786 & $1.21 \mathrm{E}-04$ & $5.59 \mathrm{E}-03$ & YPL182C & Dubious ORF \\
\hline YBR058C & 0.858321 & 8.38698 & $1.21 \mathrm{E}-05$ & $1.01 \mathrm{E}-03$ & UBP14 & Ubiquitin-specific protease \\
\hline YDR158W & 0.8485721 & 7.555994 & 2.47E-03 & 4.89E-02 & HOM2 & Aspartic beta semi-aldehyde dehydrogenase \\
\hline YIL093C & 0.8481479 & 8.633073 & 2.09E-06 & $2.58 \mathrm{E}-04$ & RSM25 & Mitochondrial ribosomal protein of the small subunit \\
\hline
\end{tabular}


Table 4.8 Cont'd

\begin{tabular}{|c|c|c|c|c|c|c|}
\hline & $\log \mathrm{FC}$ & $\log \mathrm{CPM}$ & PValue & FDR & Gene & Biological Function \\
\hline YCL005W & 0.8322289 & 6.525532 & $1.68 \mathrm{E}-03$ & $3.78 \mathrm{E}-02$ & LDB16 & Protein involved in lipid droplet assembly \\
\hline YDR391C & 0.8199758 & 7.870653 & 9.87E-05 & $5.00 \mathrm{E}-03$ & YDR391C & Putative protein of unknown function \\
\hline YPL150W & 0.8107782 & 7.097978 & $3.35 \mathrm{E}-05$ & $2.34 \mathrm{E}-03$ & YPL150W & Protein kinase of unknown cellular role \\
\hline YIL112W & 0.8104066 & 8.153484 & 8.47E-05 & $4.48 \mathrm{E}-03$ & HOS4 & Subunit of the Set3 complex \\
\hline YPL202C & 0.8015511 & 7.422982 & $1.81 \mathrm{E}-03$ & $4.01 \mathrm{E}-02$ & AFT2 & Iron-regulated transcriptional activator \\
\hline YOR371C & 0.7975345 & 7.529178 & $3.31 \mathrm{E}-04$ & $1.20 \mathrm{E}-02$ & GPB1 & Multistep regulator of cAMP-PKA signalling \\
\hline YBL013W & 0.7941395 & 7.664035 & 2.14E-04 & $8.56 \mathrm{E}-03$ & FMT1 & Methionyl-tRNA formyltransferace \\
\hline YCR006C & 0.7802903 & 7.32165 & $7.66 \mathrm{E}-04$ & $2.15 \mathrm{E}-02$ & YCR006C & Dubious ORF \\
\hline YHR206W & 0.7698855 & 8.578876 & $3.25 \mathrm{E}-04$ & $1.19 \mathrm{E}-02$ & SKN7 & Nuclear response regulator and transcription factor \\
\hline YDR096W & 0.753302 & 8.4082 & $7.71 \mathrm{E}-05$ & 4.27E-03 & GIS1 & Histone demethylase and transcription factor \\
\hline YPL148C & 0.7516617 & 8.650217 & $5.28 E-07$ & $9.20 \mathrm{E}-05$ & РPT2 & Phosphopantethrein:protein transferase \\
\hline YJL017W & 0.7416848 & 8.674153 & $2.02 \mathrm{E}-03$ & 4.27E-02 & $<\mathrm{NA}>$ & Merged ORF \\
\hline YIL123W & 0.7075129 & 8.333465 & $1.18 \mathrm{E}-04$ & $5.53 \mathrm{E}-03$ & SIM1 & Protein of the SUN family \\
\hline YBR148W & 0.699351 & 8.701616 & 8.63E-06 & $7.75 \mathrm{E}-04$ & YSW1 & Protein required for normal prospore membrane formation \\
\hline YOR267C & 0.6864597 & 8.614525 & $3.50 \mathrm{E}-04$ & $1.25 \mathrm{E}-02$ & HRK1 & Protein kinase \\
\hline YMR075C-A & 0.6839848 & 8.173032 & 1.64E-04 & $7.00 \mathrm{E}-03$ & YMR075C-A & Dubious ORF \\
\hline YMR037C & 0.6818021 & 8.285859 & $1.56 \mathrm{E}-05$ & $1.28 \mathrm{E}-03$ & MSN2 & Stress-responsive transcriptional activator \\
\hline YGL194C & 0.6774102 & 7.898106 & $1.05 \mathrm{E}-04$ & $5.23 \mathrm{E}-03$ & HOS2 & Histone-deacetylase and subunit of Set 3 and Rpd3L complexes \\
\hline YDR259C & 0.674842 & 9.130055 & $2.08 \mathrm{E}-05$ & $1.56 \mathrm{E}-03$ & YAP6 & Transcriptional factor \\
\hline YJL168C & 0.6564513 & 7.212665 & 2.18E-03 & 4.53E-02 & SET2 & Histone methyltransferase with a role in transcriptional elongation \\
\hline YLL015W & 0.6508324 & 8.273196 & $6.41 \mathrm{E}-04$ & $1.88 \mathrm{E}-02$ & BPT1 & ABC type transmembrane transport of MRP/CFTR family \\
\hline YLR304C & 0.6239353 & 7.395869 & 8.88E-04 & $2.37 \mathrm{E}-02$ & ACO1 & Aconitase \\
\hline YNL116W & 0.6238826 & 8.487863 & $4.90 \mathrm{E}-05$ & $3.05 \mathrm{E}-03$ & DMA2 & Ubiquitin-protein ligase (E3) \\
\hline YPL203W & 0.6201166 & 7.955273 & $9.36 \mathrm{E}-04$ & $2.44 \mathrm{E}-02$ & TPK2 & cAMP-dependent protein kinase catalytic subunit \\
\hline YHR163W & 0.6198133 & 7.620024 & $3.23 \mathrm{E}-04$ & 1.19E-02 & SOL3 & 6-phosphogluconolactonase \\
\hline YGL151W & 0.6183187 & 8.184705 & $1.24 \mathrm{E}-03$ & $3.00 \mathrm{E}-02$ & NUT1 & Component of the RNA polymerase II mediator complex \\
\hline YNL105W & 0.6155233 & 8.708582 & $3.48 \mathrm{E}-05$ & 2.37E-03 & RRT16 & Dubious ORF \\
\hline YLR393W & 0.6132971 & 7.785249 & $9.55 \mathrm{E}-04$ & $2.48 \mathrm{E}-02$ & ATP10 & Assemb ly factor for the F0 sector of mitochondrial F1F0 ATP synthase \\
\hline YJL057C & 0.6105927 & 7.989382 & 8.98E-04 & $2.39 \mathrm{E}-02$ & IKS1 & Protein kinase of unknown cellular role \\
\hline
\end{tabular}


To validate the Bar-seq results, we grew the 90 homozygous gene deletion strains with the lowest $\log F C$ values $(F D R<0.05)$ in the presence and absence of the methanolic extract $(0.0002 \%$ $\mathrm{v} / \mathrm{v}$ ) compared to the growth of the BY4743 WT strain. Of the 90 strains, 30 exhibited significantly reduced growth in the methanolic extract (Fig 4.8). The most hypersensitive gene deletion detected with approximately $80 \%$ growth inhibition compared to WT growth inhibition at $10 \%$ was $H D A 3$, a component of the HDA1 histone deacetylase complex (Carmen et al., 1996). The deletion of RPL12A, the $12 \mathrm{~A}$ subunit of the $60 \mathrm{~S}$ ribosomal subunit (Planta \& Mager, 1998), was found to be the next most hypersensitive gene deletion, exhibiting approximately $70 \%$ growth inhibition. Other gene deletions significantly sensitive to the methanolic extracts were involved in ribosomal structure and biogenesis (RPL19B, RPP1B, RPL37A), GTPase activation (YPT7, GYP1, MOG1, LST4, SAC7), and also calcium, sodium and phosphate transport (FLC2, PHO89). Gene deletions related to the iron chelation phenotype in Chapter 3 were also recovered, such asDAP1, WHI2, BUD14, DID2 and NAP1. These gene deletions are particularly interesting since the homozygous diploid deletion strains (this chapter) and the haploid deletion strains (Chapter 3 ) independently identified iron metabolism as a mechanism of action of $P$. insularum extracts.

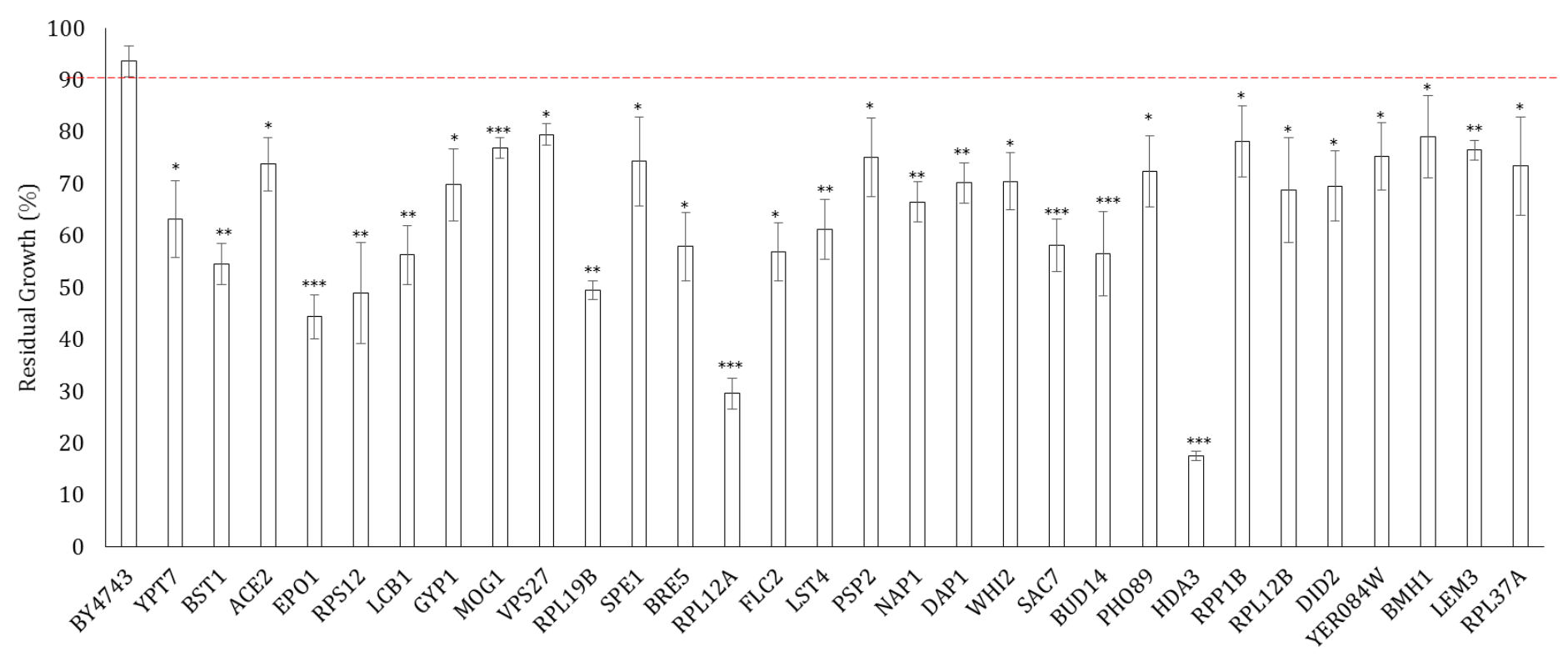


Figure 4.8: Validations of the HOP hits of the methanolic extract of $\boldsymbol{P}$. insularum. The 90 gene deletions with the lowest $\log \mathrm{FC}$ values were selected for liquid-based growth validations, the residual growths of which were compared to the BY4743 WT control. The red line indicates the $90 \%$ residual growth mark for the WT control, and the 30 gene deletions plotted were those with residual growths less than $90 \%$ consistently across the three triplicates are plotted, reported is mean residual growth \pm SD. Plotted is the average across the three triplicates, the error bars signifying the standard deviation. The $\mathrm{p}$ values were calculated using a one tailed Student's t-test $\left(*\right.$ : p-value $<0.05,{ }^{* *}$ : p-value < 0.01, ***: p-value $<0.001)$. 


\subsection{Discussion}

This chapter employed genome-wide, barcode sequencing-based HIP-HOP analyses to elucidate the genes and pathways regulated by the aqueous and methanolic extracts of $P$. insularum. Homozygous and heterozygous gene deletions were identified as hypersensitive to the extracts based on barcode sequence abundance. Specifically, the HIP analysis of the aqueous extract of $P$. insularum identified a total 51 mutants with altered growth, 25 of which were identified as hypersensitive, while 26 were identified as having improved growth in the presence of the extract. Similarly, the HIP analysis of the methanolic extract also identified 51 mutants with altered growth, although 20 were identified as hypersensitive, with the remaining 31 exhibiting positive logFC indicative of improved growth. The HOP analysis of the aqueous extract produced 30 genes with affected growth in the presence of the aqueous extract of $P$. insularum, with 18 having reduced growth, and 12 with improved growth. Contrastingly, the HOP analysis of the methanolic extract identified a large number of 307 gene deletions with affected growth, 182 of which were hypersensitive, 106 of which exhibited improved growth. However, the results for the heterozygous mutants did not validate when the strains were grown independently in contrast to the competitive pool condition in the Bar-seq analysis. Intriguingly, growth defects of 30 of the top 90 homozygous mutants with negative logFC were validated when grown independently.

Our lack of identifying the physical targets of the extracts in the HIP analyses is possibly the consequence of employing too high a concentration for the Bar-seq analyses. Screening a drug against its drug target at too high of a concentration risks the failure to identify the drug target. For example, TUB2, the known target of benomyl was not identified when benomyl was screened at $50 \mu \mathrm{g} / \mathrm{mL}\left(I_{90}\right)$ although it was identified when the heterozygous library was screened at $25 \mu \mathrm{g} / \mathrm{mL}$ $\left(I C_{20}\right)$ (Giaever et al., 1999). Likewise, when tunicamycin was screened at $2.0 \mu \mathrm{g} / \mathrm{mL}\left(I C_{80}\right)$, its target ALG7 was not identified because it was equally hypersensitive as the WT control, although it was identified when the concentration for the screen was reduced to $0.5 \mu \mathrm{g} / \mathrm{mL}\left(\mathrm{IC}_{20}\right)($ Giaever et al., 1999). However, it is unlikely that this is the case observed here, as consideration was taken to 
ensure that $\mathrm{IC}_{20}$ concentrations of the aqueous and methanolic extracts of $P$. insularum were employed in our Bar-seq analyses. Alternatively, it is plausible that based on work carried out in Chapter 3, the bioactive components of the $P$. insularum extracts do not possess a genetic target, as the extracts directly bind iron.

Interestingly, despite the lack of a genetic target identified from these HIP analyses, the additional gene deletions that were identified from the HOP analyses are buffering mechanisms that include mechanisms related to the iron chelating mechanism of action identified in chapter 3 . For instance, ERG2 involved in the heme-dependent pathway of ergosterol synthesis, was found to be significantly hypersensitive to the aqueous and methanolic extracts. Although this may be a consequence of reduced membrane rigidity resulting from defective ergosterol biosynthesis under low iron conditions (Abe \& Hiraki, 2009; Shakoury-Elizeh et al., 2010), it is also likely that this hypersensitivity is a consequence of the vacuolar defect of the erg2 $\Delta$ mutant, as mutants with vacuolar defects are hypersensitive under iron deficient conditions (Beh \& Rine, 2004; Szczypka et al., 1997). Furthermore, our results are consistent with a relationship between iron-sulfur proteins and ribosomal proteins (Yarunin et al., 2005) wherein a deletion of the iron-sulfur cluster requiring protein Rli1p leads to defective processing of pre-rRNA, as well as defective export of both $40 \mathrm{~S}$ and 605 ribosomal subunits. This link may explain why genes involved in ribosomal biogenesis, ribosomal subunits and ribosomal assembly were detected from our genome-wide analyses with the extracts of $P$. insularum (e.g., JJJ1 from aqueous extract HOP, RPL10 from methanolic extract HIP andRPS12 and RPL12A from methanolic extract HOP), our logic being that a defect in iron-sulfur cluster synthesis resulting from reduced iron content upon treatment with the extracts of $P$. insularum exacerbates a defect already brought about by the deletion of subunits of the ribosomal machinery (e.g., RPL12A and RPS12).

The gene deletion bre5 $\Delta$ was found to be hypersensitive to the methanolic extract. The Ubp3p/Bre5p ubiquitin protease complex functions in various cellular processes including telomeric silencing, transcriptional regulation, cell wall integrity, autophagy and DNA damage (Bilsland et al., 
2007; Kraft et al., 2008). While a buffering link between these processes and the iron chelation activity of the extracts is not apparent, it is likely that iron chelation exacerbates these phenotypes resulting from the above gene deletions. For instance, iron chelation can reduce the effectiveness of iron-dependent proteins in their function to repair damaged DNA (Shakoury-Elizeh et al., 2004), which coupled with the deletion of BRE5 can lead to accumulated DNA damage resulting in hypersensitivity.

Another gene of interest arising from the HOP analysis of the methanolic $P$. insularum extract is DAP1, which encodes a heme-binding protein in which mutations of which result in

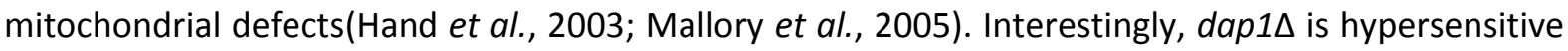
under iron-limiting conditions, possibly due to a defect in vacuolar morphology, which was previously reported to cause hypersensitivity in iron limited conditions (Craven et al., 2007; Szczypka et al., 1997). The same can be proposed for did2 $\Delta$ involved in the vacuolar protein sorting pathway (Amerik et al., 2000).

The homozygous deletion of HDA3, a subunit of the HDA1 histone deacetylase complex (Carmen et al., 1996), conferred the greatest hypersensitivity to the methanolic extract of $P$. insularum. This result suggests that the homeostasis between histone acetylation and deacetylation is disrupted by one or multiple compounds of the crude methanolic extract. Interestingly, the deletion of HDA3 appears more sensitive against the methanolic extract of $P$. insularum at $0.0002 \%$ $\mathrm{v} / \mathrm{v}$ with a growth inhibition of $\sim 80 \%$, compared to the deletion of iron transporters FET3 and FTR1 which exhibited a $20 \%$ reduction in growth at similar concentrations. This may suggest fewer compensating pathways for histone acetylation than iron transport, or it may be a consequence of the different backgrounds at which these two growth inhibition levels were obtained as the HDA3 deletion was diploid and the FET3 and FTR1 deletions were haploid. The homozygous deletion of NAP1, which is critical to the function of histone chaperones (Ishimi \& Kikuchi, 1999), was also found to be hypersensitive to the methanolic extract of $P$. insularum. Intriguingly, iron deprivation induced by curcumin treatment led to modifications in histone acetylation and degradation of Sml1p, an 
inhibitor of ribonucleotide reductase that also functions in regulating dNTP production (Azad et al., 2013; Chabes et al., 1999; Zhao et al., 1998). A similar cumulative effect of iron deprivation on dNTP levels could explain why $h d a 3 \Delta$ and nap1 $\Delta$ were hypersensitive to $P$. insularum extract treatment in our HOP analysis. Additionally, given these findings, further research is required to investigate the possibility of the methanolic $P$. insularum extract being a natural histone deacetylase (HDAC) inhibitor. From a different perspective, given the nature of some iron chelators to also chelate $\mathrm{Zn}$, it may not be surprising that $P$. insularum extracts induced a HDA3 response if it were a $\mathrm{Zn}$ chelator as well, given that $\mathrm{Zn}$ regulates some histone deacetylases (Tan \& Liu, 2015). However, our ICP-MS data (Appendix VIII) and metal-rescue data (Fig 3.9) suggested that extracts of $P$. insularum do not chelate $\mathrm{Zn}$. For instance, ICP-MS data showed that there was no significant increase or decrease in Zn levels when cells were treated with the extracts. Furthermore, addition of $\mathrm{Zn}$ back into media with extracttreated cells did not partially rescue the growth defect induced by the extracts, suggesting that no part of the growth defect observed was attributable to any $\mathrm{Zn}$ chelation.

Intriguingly, the profile obtained from this chapter shared some similarities to the findings from the haploid screen carried out in the preceding chapter. In particular, both sets of screens did not identify the iron-sensing transcription factors or transporters the various iron transport systems FET4 excluded. Although this was identified to be an inherent caveat of agar screening in this particular instance (i.e., we probed transporter and transcription factor deletion mutants on agar against the aqueous extract but the mutants did not appear hypersensitive), whether this is the case in liquid needs to be clarified, by validating the diploid strains (both heterozygous and homozygous) of the iron transporters and the transcription factors. Additionally, both sets of screens identified genes involved in ribosomal biogenesis, ribosomal subunits and ribosomal assembly. This indicates an intricate relationship between iron and these cellular processes that warrants further investigation. 
Although our HIP analyses did not identify genetic targets for either the aqueous or the methanolic extracts of $P$. insularum, and albeit the iron transporters detected in the agar-based genomic analysis in Chapter 3 were not detected in the HOP analyses in this chapter, we used these Bar-seq analyses to identify new pathways that were regulated by the extracts of $P$. insularum as well as present evidence of their complementation of data in Chapter 3. For instance, ribosomal biogenesis and organization was detected from these analyses that were not previously identified in the agar-based analysis in Chapter 3. Furthermore, a possible link with histone acetylation/deacetylation was also detected here, that was not detected in Chapter 3, but is consistent with the acetylation/deacetylation effect of the iron chelator curcumin previously reported (Azad et al., 2013). For future work, characterization of the validated hits to determine their mechanistic role under extract treatment is required to fully comprehend the underlying molecular response to the $P$. insularum extracts. These investigations will also provide information on iron metabolism, regulation of iron homeostasis and how cells respond to changing iron status particularly in the presence of an iron chelator. Furthermore, investigations into the gene deletions that conferred better growth in the presence of the $P$. insularum extracts will also be helpful in identifying genes that can improve growth in the absence of iron for normal cellular processes. In conclusion, this chapter provided complementary findings that we have correlated to the proposed iron chelating mechanism of action of the $P$. insularum extracts. 


\section{Chapter 5:}

\section{Identification of the Iron Chelating Condensed Tannin from the Aqueous Extract of Psychotria insularum}

\subsection{Introduction}

The preceding chapters described our work in ascertaining the mechanism of action of $P$. insularum extracts and associated buffering mechanisms without any knowledge of the bioactive compound or compounds. While that part of the work was highly crucial, it was also of great importance to determine the identity of the responsible bioactive compound, to assess its novelty and potential for pharmaceutical exploitation. Plant secondary metabolites are interesting sources of bioactive compounds, and they represent a hugely diverse group of compounds that are not usually necessary for plant cell structure and function, but are produced as part of the plant defence system against stresses and contribute to plant pigmentation (Rispail et al., 2005). Secondary metabolites include terpenoids, alkaloids and phenolics, some of which are of therapeutic significance, currently used or undergoing trials for the prevention or treatment of several diseases such as respiratory infection, anxiety disorders, sleep disorders, autoimmune diseases, inflammatory bowel diseases, Alzheimer's disease and various types of cancer (Raskin et al., 2002; Reddy et al., 2003; Watson et al., 2001). Indeed, condensed tannins, epicatechins and flavanols possess an iron binding moiety which bears resemblance to the catechol motif that has been shown to bind iron (Baker et al., 2003; Heim et al., 2002). However, natural iron chelators are chemically diverse, and may also include iron siderophores that iron-dependent microorganisms have evolved to secrete to scavenge iron from their surrounds like the well-known iron chelator Deferoxamine (DFX) in clinical use (Hatcher et al., 2009). There is also gathering evidence of phytochemicals, chemicals of plant origin, being potent antioxidants and iron chelators (Hatcher et al., 2008; Hider et al., 2001; Kell, 2009; Morel et al., 1993). 
Bioassay guided fractionation is a compound isolation technique that makes use of the step-wise fractionation of extracts directed by the bioactivity of the fractions obtained from each fractionation step (Fig 5.1). Indeed, this fractionation approach has led to the isolation and identification of bioactive compounds from crude plant extracts. For instance, $\alpha$-glucosidase inhibitors were successfully isolated and identified following bioassay-guided fractionation of leaf extracts from the plant Aquilaria sinensis, with a potential clinical significance in the therapy of diabetes (Feng et al., 2011). Additionally, fractionated compounds from the crude leaf extracts of the garden croton (Codiaeum variegatum) used by Cameroons to treat bloody diarrhoea, were shown to be bioactive, via a unique mechanism of action, by targeting ceramide, a lipid involved in cell membrane function (Njoya et al., 2014). The antibacterial compound okundoperoxide was isolated from crude extracts of the medicinal plant Scleria striatinux following a bioassay guided fractionation approach (Mbah et al., 2012). Most importantly, this bioassay guided fractionation approach was used to isolate compounds bearing the iron-binding catechol moiety from flowers of the Spartium junceum plant used in Turkish folk medicine (Yeşilada et al., 2000).

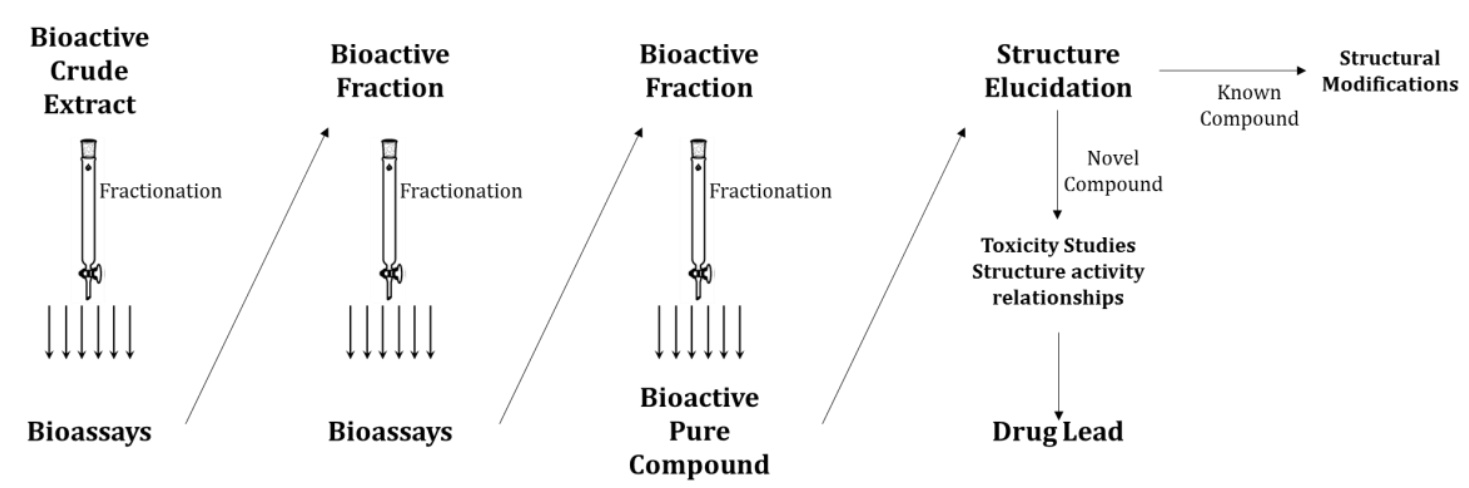

Figure 5.1: Schematic of bioassay guided fractionation. Further purification of each subsequent fraction is dictated by its bioactivity.

Given the success of bioassay guided fractionation of crude medicinal plant extracts to isolate bioactive compounds (some of which with iron chelating capacity), this approach was adopted for the determination of the bioactive component of the crude aqueous extract of $P$. 
insularum leaves responsible for its iron chelation activity. Our approach incorporated yeast growth inhibition and iron chelation assays to track the compound responsible for the iron rescue activity. Polar fractionation and size-exclusion chromatography were used for the fractionation of our aqueous extract. In conjunction with our bioassays, NMR spectra were also obtained. Further, the protein expression profiles of the high affinity iron transporters Fet3p and Ftr1p were evaluated in the presence of the fractions of interest, via monitoring the GFP intensity of their GFP-tagged counterparts. We found that the fractions with the iron chelating activity of interest tracked to a 67ppm resonance using ${ }^{1} \mathrm{H}$ NMR, which was subsequently determined to be a condensed tannin. Further, the expression levels of the iron transporters were up-regulated in the presence of the fractions displaying bioactivity, similar to the result obtained when cells were treated with the crude extracts. 


\subsection{Methods}

\subsubsection{HP20 column chromatography fractionation}

Supelco Diaion ${ }^{\circledast}$ HP20 resin columns were prepared based on the principle that $1 \mathrm{~mL}$ of HP20 resin binds approximately $25 \mathrm{mg}$ of extractable compounds. The aqueous extract of $P$. insularum was passed through the column four times at a flow rate of $4 \mathrm{~mL} / \mathrm{min}$. The HP20 column was eluted with three column volumes of $30 \%, 75 \%$ and $100 \%$ acetone in water. The $100 \%$ acetone fraction was evaporated under reduced pressure while the $30 \%$ and $75 \%$ fractions were subjected to backloading.

\subsubsection{Backloading}

Backloading of fractions eliminates long evaporating times particularly with samples of larger volumes and reduces bumping arising from samples containing water such as those eluted with $30 \%$ and $75 \%$ acetone/methanol in water. The $30 \%$ and $75 \%$ fractions that required backloading were diluted two-fold with $\mathrm{dH}_{2} \mathrm{O}$ before passing them through a column with half the column volume of resin as the previous fractionation step. Fractions were diluted two-fold and passed through the column to a final concentration of $12.5 \%$ acetone. The column was eluted with three column volumes of $100 \%$ acetone, essentially exchanging the aqueous solvent elution to a purely organic solvent, and dried under pressure as previously described. The mass of the fraction was determined by solubilising in methanol: dichloromethane $(4: 1)$, followed by transferring and drying into a pre-weighed vial.

\subsubsection{HP20ss column chromatography fractionation}

Columns for HP20ss fractionation were prepared similar to HP20 columns at a ratio of $1 \mathrm{~mL}$ of resin to $25 \mathrm{mg}$ extractable compounds. Loaded HP20ss was transferred to a larger column bed of 
HP20ss (nine times the volume of the loaded resin). Columns were eluted with the relevant acetone concentration as described in specific sections.

\subsubsection{LH20 column chromatography fractionation}

LH20 size-exclusion chromatography fractionation was achieved by soaking $100 \mathrm{~mL}$ of LH20 resin in $90 \%$ methanol in water overnight before set up. The relevant fraction was passed through the column at approximately $0.3 \mathrm{~mL} / \mathrm{min}$ flow rate, and $5 \mathrm{~mL}$ fractions were collected in $10 \mathrm{~mL}$ test tubes ( $330 \times 5 \mathrm{~mL}$ fractions) using an automated fraction collector. To ensure all material was eluted, $2 \times 1 \mathrm{~L}$ bulk collections were subsequently collected.

\subsubsection{Thin Layer Chromatography (TLC)}

To determine the complexity of samples and to ascertain those with similar composition, TLC analyses were carried out to permit the pooling of multiple samples in order to reduce the number of samples to be subsequently assayed for bioactivity and NMR spectroscopy. TLC was performed using Polygram ${ }^{\circledast}$ SilG/UV254 plates (Machery-Nagel) in $30 \%$ methanol in dichloromethane as the running solvent, followed by $0.1 \% \mathrm{w} / \mathrm{v}$ vanillin in ethanol, heated for analyses, and visualized under UV light (254 nm and $350 \mathrm{~nm}$ ).

\subsubsection{Nuclear Magnetic Resonance spectroscopy}

NMR spectra were acquired using a Varian DirectDrive spectrometer with triple resonance $\mathrm{HCN}$ cryogenic probe operating at $600 \mathrm{MHz}$ for ${ }^{1} \mathrm{H}$ using deuterated methanol (Cambridge Isotope Laboratories Inc). 


\subsubsection{Tannin test}

The tannin test was conducted as previously described (Dhandapani \& Sabna, 2008). Briefly, approximately $2 \mathrm{mg}$ of a fraction was dissolved in $3 \mathrm{~mL}$ methanol, to which three drops of $100 \mathrm{mM} \mathrm{FeCl}_{3}$ was added. The sample colour was then observed ranging from a brownish green to blue-black colouration.

\subsubsection{Condensed tannin test}

The hydrolysis of proanthocyanidin by condensed tannin assay was conducted as previously described (Porter et al., 1986). Concisely, butanol:hydrochloric acid (95:5 v/v) solution was added to the fraction, leading to the auto-oxidation and hydrolysis of proanthocyanidin, detected as the red coloured anthocyanidin monomer precipitate.

\subsubsection{Bioactivity assay}

Liquid-based growth assays of bioactivity were carried out as previously described in Section 2.2.3.

\subsubsection{Iron supplementation assay}

Rescue of growth defects via iron supplementation was carried out as previously described in Section 3.2.4.

\subsubsection{Protein expression}

Monitoring of the abundance of GFP-tagged proteins was carried out as described previously in Section 3.2.6. 


\subsection{Results}

\subsubsection{The $30 \%$ and $75 \%$ acetone fractions are bioactive}

The first fractionation step of the aqueous extract of $P$. insularum involved reversed-phase column chromatography utilizing HP20 resin. Fractions were eluted with 30\%, 75\% and $100 \%$ acetone in water. Upon evaporation of the samples, it was determined that the $100 \%$ fraction did not contain sufficient material for bioassays. The fractionated $30 \%$ and $75 \%$ material was solubilized in DMSO and assayed for bioactivity as well as the iron rescue assay to determine if they retained the bioactivity profile described in the preceding chapter. The $30 \%$ and $75 \%$ fractions of the aqueous extract of $P$. insularum reduced the growth of BY4741 WT yeast strain (Fig 5.2). At their lowest tested concentration of $6.4 \times 10^{-5} \% \mathrm{v} / \mathrm{v}$, the $30 \%$ HP20 fraction inhibited growth of BY4741 by approximately $15 \%$, while the $75 \%$ HP20 fraction inhibited growth of BY4741 by approximately $30 \%$, compared to the control. Interestingly, both fractions reduced growth of BY4741 by close to $100 \%$ at $1.6 \times 10^{-3} \% \mathrm{v} / \mathrm{v}$. Supplementation with $100 \mu \mathrm{M}$ exogenous $\mathrm{FeCl}_{3}$ rescued the growth defect induced by both fractions, suggesting that the bioactivity determined from treatment with the fractions alone is mediated by the iron chelation mechanism that was previously established in Chapter 3. Further, these findings indicate that both the $30 \%$ and $75 \%$ fractions contain the bioactive, iron chelating component.
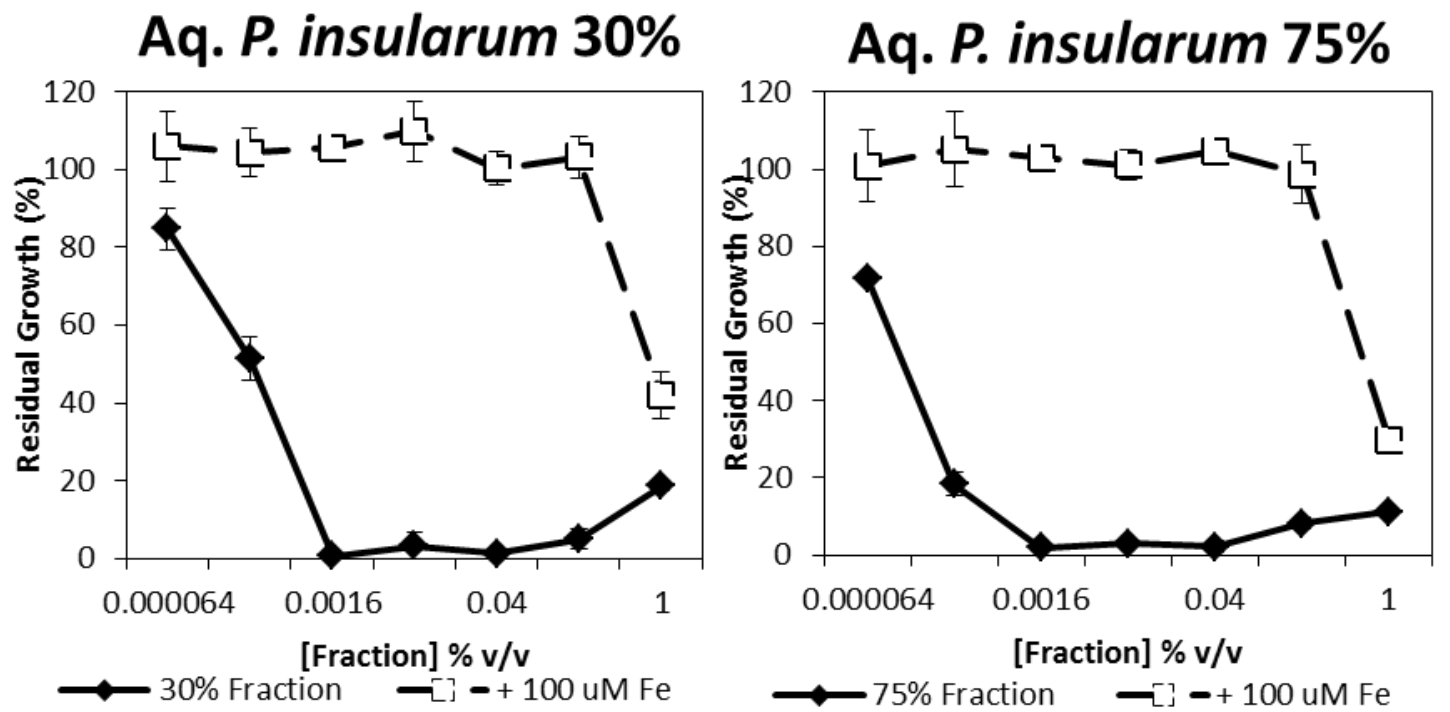
Figure 5.2: Bioassays of the $30 \%$ and $75 \%$ HP20 fractions of the aqueous $P$. insularum extract. Liquid based bioactivity and iron rescue assays of the 30\% and 75\% HP20 fractions of the aqueous extract of $P$. insularum was achieved by exposing BY4741 WT yeast to increasing concentrations of the fractions, and grown with (open squares) or without (closed diamonds) supplementation with $100 \mu \mathrm{M}$ $\mathrm{FeCl}_{3}$. Residual growth was obtained by comparing the growth of treated cells against DMSO control. The solid black lines indicate treatment with the fraction alone, while the dashed lines indicate fraction treatment with $100 \mu \mathrm{M} \mathrm{FeCl}_{3}$ supplementation.

\subsubsection{The ${ }^{1} \mathrm{H}$ NMR analyses of the $30 \%$ and $75 \%$ fractions from $\mathrm{HP} 20$ fractionation show similar spectra profiles}

${ }^{1} \mathrm{H}$ NMR spectroscopy is a technique that provides information regarding the chemical composition of a given sample based upon the different 3D spatial environments that each hydrogen atom exists in the so-called "chemical environment". In this work, NMR spectroscopy was employed to complement the bioassay-guided fractionation, to provide insight into the possible composition and complexity of the fractions. As such, sub-samples of the bioactive $30 \%$ and $75 \%$ HP20 fractions were solubilized in deuterated methanol and run at $600 \mathrm{MHz}$ for ${ }^{1} \mathrm{H}$ using a Varian DirectDrive spectrometer. The spectra of $30 \%$ and $75 \%$ HP20 fractions showed similar profiles (Fig 5.3), a result that is consistent with these fractions exhibiting similar bioactivity profiles. Similarities between the two samples (black dashed boxes in Fig 5.3) were observed at 2-3 ppm where hydrogen atoms adjacent to double bonds often resonate, and at 6-7 ppm where alkene hydrogen atoms resonate. However, there also existed a difference between the samples. This difference (red dashed box in Fig 5.3) was noted between 3-4 ppm where the hydrogen attached to oxygenated carbon atoms (e.g., carbohydrates) normally resonate. The fact that these resonances were missing in the $75 \%$ acetone fraction imply they were irrelevant to the observed bioactivity. The large peaks observed at 4.90 ppm and $3.31 \mathrm{ppm}$ correlate to water and the residual protons of deuterated methanol, respectively. 


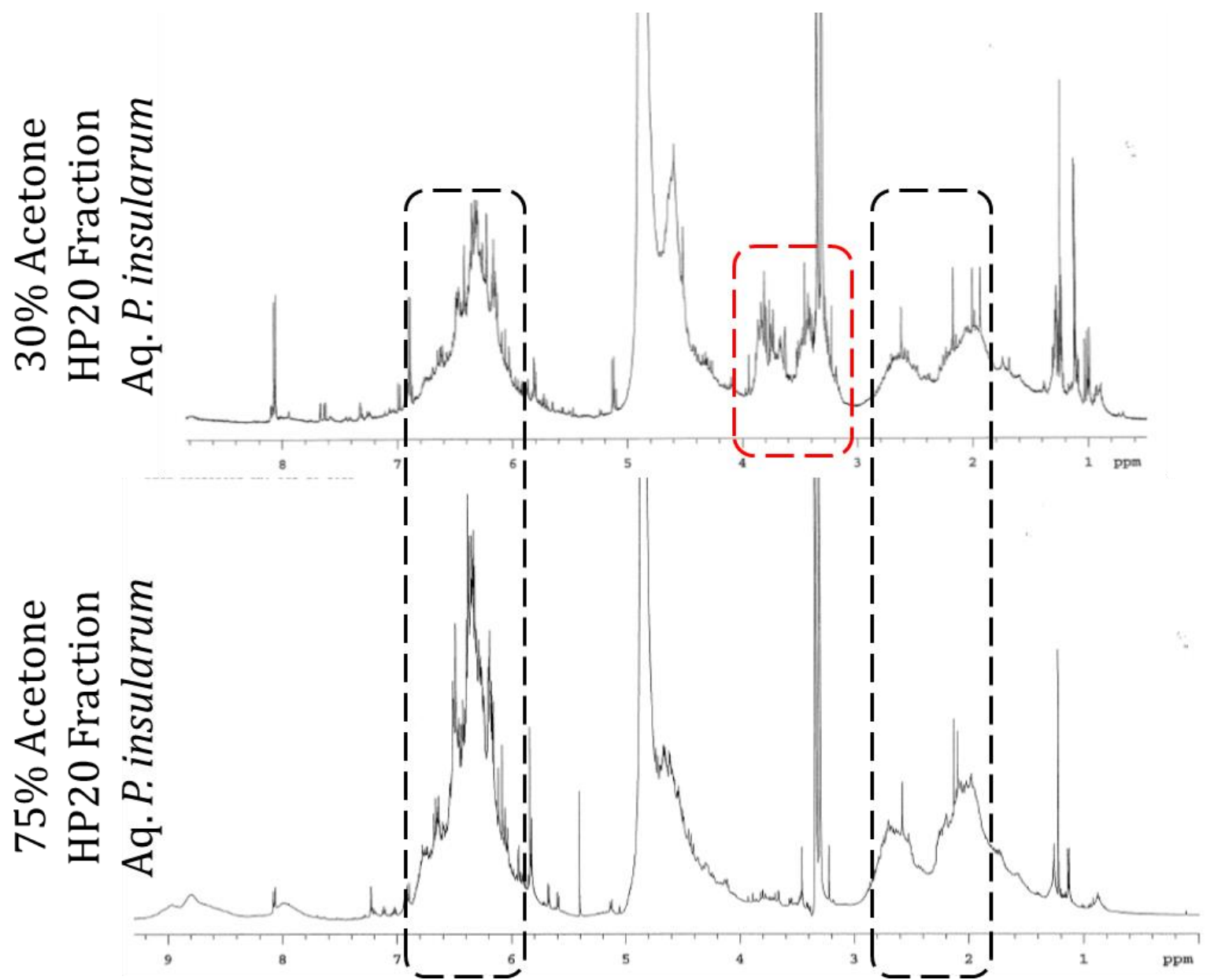

Figure 5.3: NMR spectra of the $30 \%$ and $\mathbf{7 5 \%}$ HP2O fractions of the aqueous extract of $P$. insularum. NMR spectra of the $30 \%$ and $75 \%$ HP20 fractions of the aqueous extract of P. insularum, solubilized in deuterated methanol, and obtained from a Varian DirectDrive spectrometer with a triple resonance $\mathrm{HCN}$ cryogenic probe run at $600 \mathrm{MHz}$ for ${ }^{1} \mathrm{H}$. The large peaks observed at $4.90 \mathrm{ppm}$ and 3.31 ppm correlate to water and the residual protons of deuterated methanol, respectively.

\subsubsection{Sub-fractions of HP20ss fractionation of 30\% fraction are bioactive and variably potent}

To further purify the components of the $30 \%$ HP20 fraction, it was processed through a HP20ss column and eluted with 10\%, 20\%, 30\%, 50\% and 100\% acetone in water. The fractions were backloaded and dried in vacuo before solubilizing in DMSO. Minimal material $(<0.5 \mathrm{mg})$ was obtained from the $10 \%, 20 \%$ and $30 \%$ fractions and work with these fractions thus stopped at this stage. However, to determine if the $30-50$ (50\% HP20ss fraction of the $30 \%$ HP20 fraction of the aqueous extract of $P$. insularum) and the $30-100$ (100\% HP20ss fraction of the $30 \%$ HP20 fraction of the aqueous extract of $P$. insularum) fractions were bioactive, growth inhibition and iron- 
supplementation assays were conducted against the BY4741 WT yeast strain. The $50 \%$ and $100 \%$ HP20ss fractions of the 30\% HP@0 fraction of the aqueous extract of $P$. insularum were bioactive based on inhibited growth of the yeast strain illustrated by the downward slope of the residual growth curves (solid black line in Fig 5.4). Interestingly, the 100\% HP20ss fraction caused an approximate $30 \%$ inhibition in BY4741 growth at $6.4 \times 10^{-5} \% \mathrm{v} / \mathrm{v}$, while the $50 \%$ HP20ss fraction caused an approximate $10 \%$ inhibition at the same concentration. This suggests the $100 \%$ HP20ss fraction was marginally more potent than the 50\% HP20ss fraction. Supplementation of fraction treatments with $100 \mu \mathrm{M} \mathrm{FeCl}$ greatly improved BY4741 WT growth, indicating the bioactive component responsible for the iron chelation activity is present in both fractions. Interestingly, $\mathrm{FeCl}_{3}$ rescued the $100 \%$ HP20ss fraction better than the $50 \%$ HP20ss fraction, where $100 \mu \mathrm{M} \mathrm{FeCl}$ supplementation of the $1 \% \mathrm{v} / \mathrm{v}$ concentration of the $100 \%$ HP20ss fraction treatment rescued residual growth from $20 \%$ to $80 \%$, while the same concentration of $50 \%$ HP20ss fraction treatment only produced a residual growth rescue from $30 \%$ to $50 \%$.
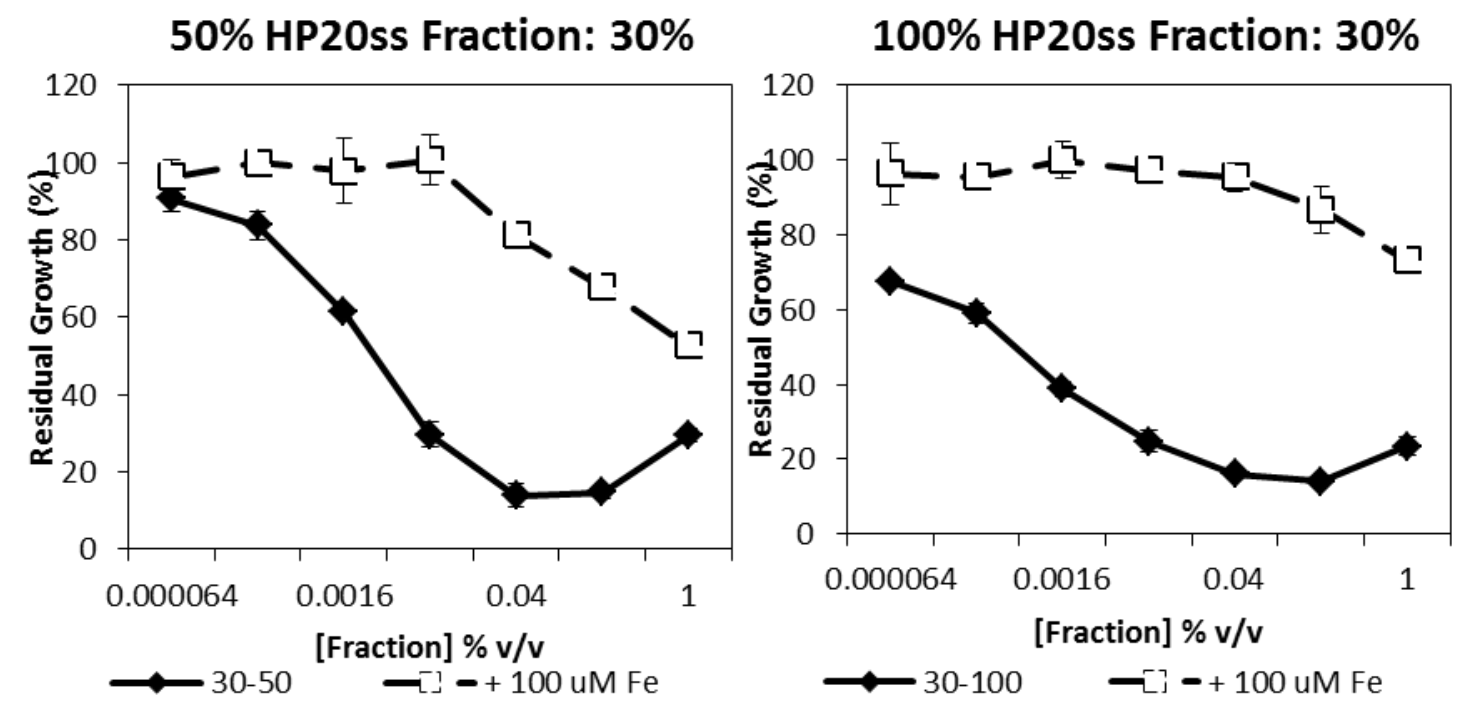

Figure 5.4: Bioactivity and iron rescue assays of the HP20ss fractions of the $30 \%$ HP20 fraction of the aqueous extract of $\boldsymbol{P}$. insularum. BY4741 WT yeast strain was grown in the presence of the 50\% (30-50) and 100\% (30-100) HP20ss fractions of the 30\% HP20 fraction, across a concentration range of $6.4 \times 10^{-5}$ to $1 \% \mathrm{v} / \mathrm{v}$. Cells were grown in either the fraction alone to determine its bioactivity (solid black line), or the fraction treatment with $100 \mu \mathrm{M} \mathrm{FeCl}_{3}$ supplementation (dashed black line) to assess iron rescue. Residual growth in each case was calculated by comparison to the DMSO control. 


\subsubsection{The NMR spectra of the $50 \%$ and $100 \%$ HP20ss fractions of the $30 \%$}

aqueous extract fraction show highly similar profiles

The activities of both the $50 \%$ and $100 \%$ HP20ss fractions as shown above suggest that both fractions contain a similar bioactive component with the iron chelation activity of interest. To assess the chemical composition of the fractions, the ${ }^{1} \mathrm{H}$ NMR spectrum of each fraction was acquired using a Varian DirectDrive spectrometer at $600 \mathrm{MHz}$ for ${ }^{1} \mathrm{H}$. The NMR spectra of the $50 \%$ and $100 \%$ HP20ss fractions from the 30\% HP20 fraction of the aqueous extract of $P$. insularum showed near identical spectral profiles (Fig 5.5). The two areas of similarity previously identified from the bioactive $30 \%$ and $75 \%$ HP20 fractions of the aqueous $P$. insularum are again present in these two fractions (highlighted in black in Fig 5.5). The area which differentiated the $30 \%$ and $75 \%$ HP20 fractions (highlighted in red) was present in both fractions although it is hypothesized that because it was uniquely present in the $30 \%$ fraction but not in the $75 \%$ HP20 fraction, it is unlikely to contribute to the bioactivity of the extracts despite its presence in both the 50 and $100 \%$ fractions. However the HP20ss fractionation did not appear to have separated out any other components of the mixture from its $30 \%$ HP20 fractionation form.

Figure 5.5: NMR spectra of the $50 \%$ and $100 \%$ HP20ss fractions from $30 \%$ HP20 fraction of the aqueous $\boldsymbol{P}$. insularum extract. NMR spectra of the $50 \%$ and $100 \%$ HP20ss fractions of the $30 \%$ HP20 fraction of the aqueous extract of $P$. insularum, solubilized in deuterated methanol, and obtained with a Varian DirectDrive spectrometer at $600 \mathrm{MHz}$ for ${ }^{1} \mathrm{H}$. The black dashed boxes indicate areas of similarity. The red box indicates the area that was unique to the $30 \%$ HP20 fraction. 


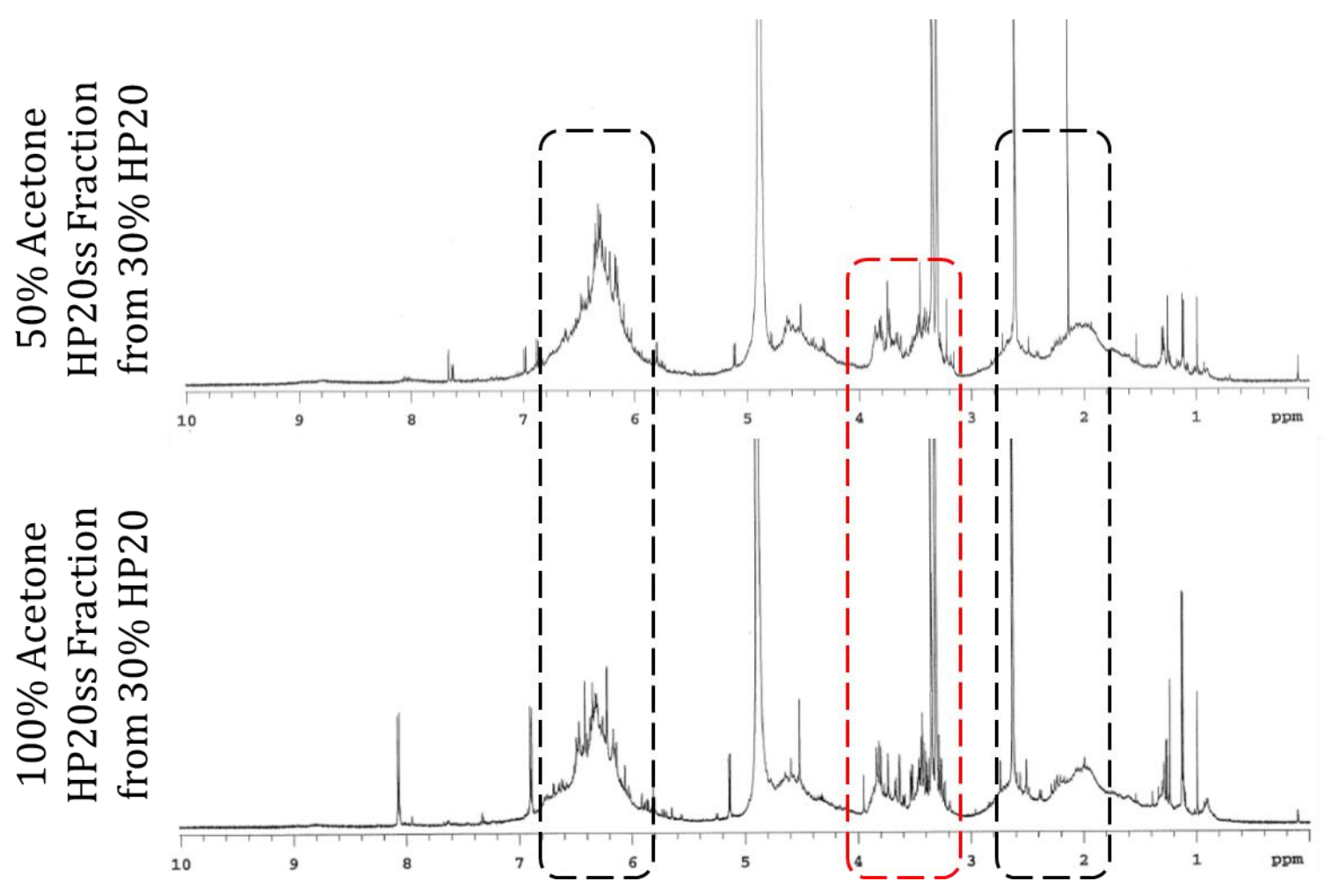

5.3.5. Sub-fractions of HP20ss fractionation of $75 \%$ fraction are bioactive and variably potent

To further purify the $75 \%$ HP20 fraction of the aqueous extract of $P$. insularum, it was loaded onto a HP20ss column and eluted with three column volumes of $30 \%, 40 \%, 50 \%, 60 \%, 75 \%$ and $100 \%$ acetone in water. TLC was performed to identify fractions that had similar chemical compositions in order to pool chemically similar samples for subsequent biological and chemical characterization of the fraction (data not shown). Samples of similar composition were pooled and evaporated in vacuo. However, insufficient material was recovered from the $30 \%$ and $40 \%$ HP20ss fractions and work with these fractions ceased. The 60\%, 75\% and 100\% HP20ss fractions however were pooled. Growth inhibition bioactivity assays and iron rescue assays were carried out on the $50 \%$ and pooled $60-100 \%$ fractions. Bioactivity assays of the $50 \%$ and $60-100 \%$ pooled fractions from the HP20ss fractionation of the 75\% HP20 fraction from the aqueous extract of $P$. insularum clearly showed bioactivity, demonstrated through the decreasing residual growth of BY4741 WT as the concentration of the fractions increased to $1 \% \mathrm{v} / \mathrm{v}$ (Fig 5.6). The potency between the two fractions was comparable as these fractions both reduced growth of the yeast cells by $90 \%$ at $0.04 \% \mathrm{v} / \mathrm{v}$ 
compared to untreated cells. Further, supplementation of both fraction treatments with $100 \mu \mathrm{M}$ $\mathrm{FeCl}_{3}$ rescued the growth defect. These findings indicate that both fractions were bioactive, and that their bioactivity was most likely due to a bioactive component with the iron chelation activity of interest.

50\% HP20ss Fraction: 75\%

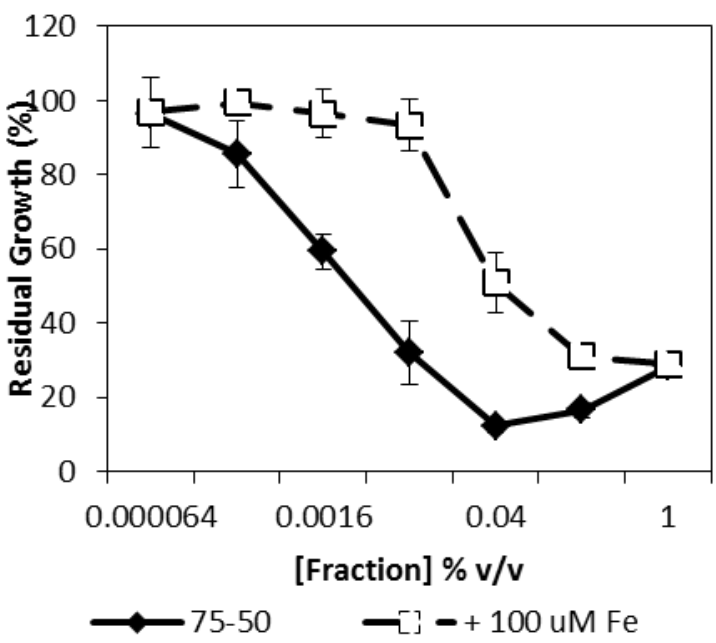

60:100\% HP20ss Fraction: 75\%

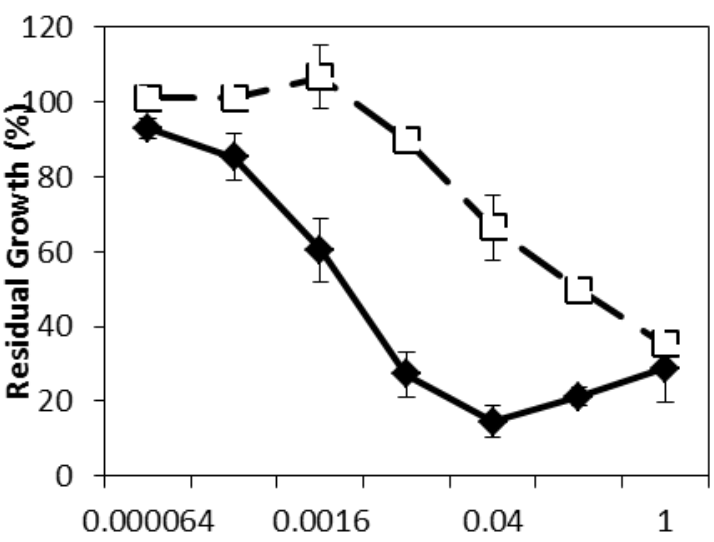

[Fraction] \% v/v

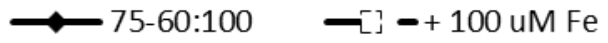

Figure 5.6: HP20ss fractions of the 75\% HP20 fraction from the aqueous extract of $P$. insularum. Bioactivity and iron rescue assays of the 50\% and 60:100\% pooled fractions from HP20ss fractionation of the 75\% HP20 fraction of the aqueous extract of $P$. insularum. Solid black lines indicate WT grown in the presence of increasing concentrations of the fractions. Dashed black lines indicate fraction treatment with $100 \mu \mathrm{M} \mathrm{FeCl}_{3}$ supplementation.

5.3.6. NMR spectra of the 50\% and $60-100 \%$ HP20ss fractions of the $75 \%$ HP20 fraction display extremely similar profiles

To obtain insight into the chemical complexity of the $50 \%$ and $60-100 \%$ fractions from the HP20ss fractionation of the $75 \%$ HP20 fraction from the aqueous extract of $P$. insularum, ${ }^{1} \mathrm{H}$ NMR spectroscopy was carried out on the two fractions at $600 \mathrm{MHz}$ for ${ }^{1} \mathrm{H}$ using a Varian DirectDrive spectrometer (Fig 5.7). Both the $50 \%$ and $60-100 \%$ pooled fractions displayed the previously observed areas of interest, at 2-3 and 6-7 ppm. These two areas have been consistently observed across all bioactive fractions, and may singly or together contribute to the iron chelation bioactivity 
of the aqueous extract of $P$. insularum. Also observed from the spectra was the apparent lack of separation of the two broad resonances of interest that have both been consistently present in the bioactive fractions, which may suggest that these peaks form part of one suite of compounds, or are from different groups of compounds but with similar polarity, owing to their co-isolation in the bioactive fractions using polarity-based fractionation.

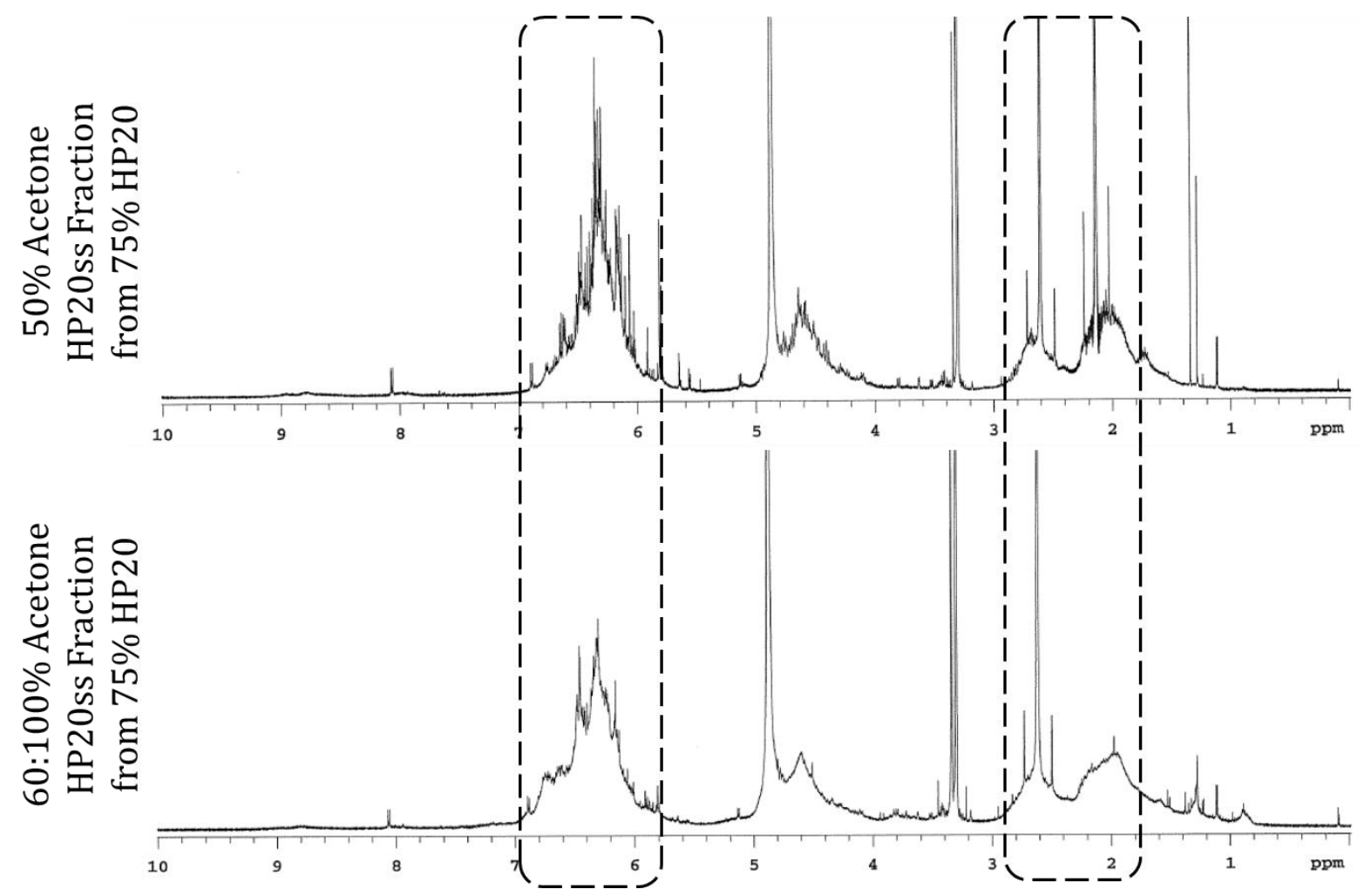

Figure 5.7: NMR spectra of the 50\% and 60:100\% HP20ss fractions of the 75\% HP20 fraction of the aqueous extract from P. insularum. Sub samples of the 50\% HP20ss fraction and the $60: 100 \%$ pooled fractions from the HP20ss fractionation of the 75\% HP20 fraction of the aqueous extract of $P$. insularum were dissolved in deuterated methanol then exposed to NMR spectroscopy using a Varian DirectDrive spectrometer at $600 \mathrm{MHz}$ for ${ }^{1} \mathrm{H}$. The black dashed lines indicate areas identified from the original 75\% HP20 fraction. 
5.3.7. The 75-50\% LH20 pooled fractions A, E, F, G, H, I, K and P are not bioactive

It was evident from the HP20ss fractionation and their respective NMR spectra that separation of extract components based on their polarity was not efficiently separating extract constituents from the bioactive component. It was thus determined that a size exclusion column be used to separate extract components by molecular size for the next step of fractionation. This approach, utilizing LH2O resin, permits larger molecular entities to pass through the column before smaller molecules are eluted due to the smaller compounds being temporarily trapped within pores on the resin surface. The attempt to further purify the iron chelating bioactive compound of $P$. insularum extract via LH20 size exclusion column chromatography was carried out on the $50 \%$ HP20ss fraction of the $75 \%$ HP20 fraction from the aqueous extract of $P$. insularum. This partial purification approach produced $3305 \mathrm{~mL}$ eluents, which were pooled to eight fractions. Growth inhibition bioactivity assay and NMR spectral analyses were conducted for the eight pooled fractions, from which it was determined that none of the eight pooled fractions was able to inhibit yeast growth sufficiently past the $80 \%$ residual growth cut-off at the $0.0001 \% \mathrm{v} / \mathrm{v}, 0.0005 \% \mathrm{v} / \mathrm{v}$ and $0.001 \% \mathrm{v} / \mathrm{v}$ concentrations (Fig 5.8). Interestingly, the NMR spectra of the 8 fractions clearly showed the absence of the peaks of interest at 2-3 ppm and 6-7 ppm, suggesting that one or both of these peaks are responsible for the iron chelation bioactivity. 

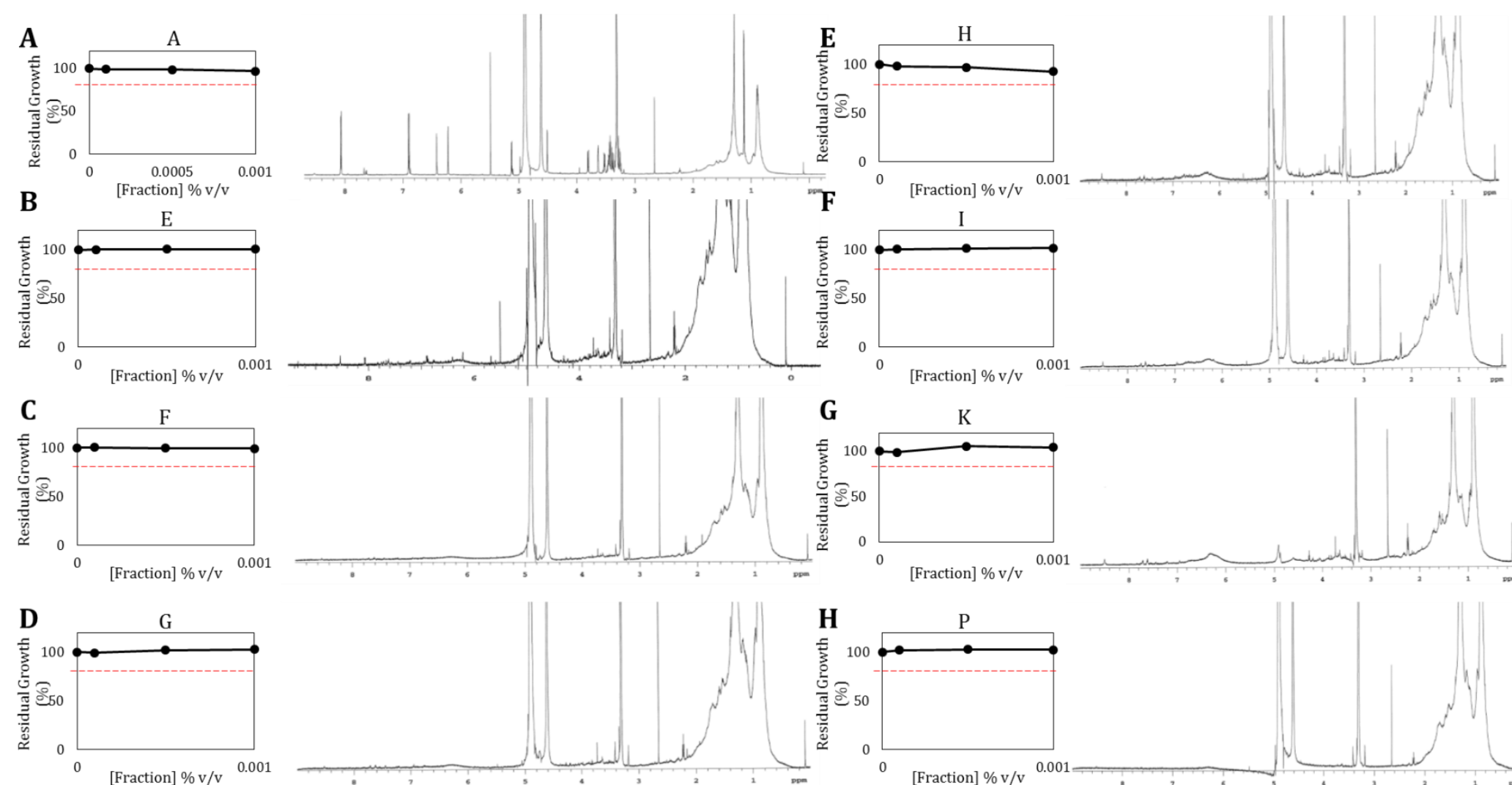

Figure 5.8:

Bioactivity assay and NMR spectra of the pooled fractions from the LH20 fractionation of the 75-50 fraction from the aqueous extract of

P. insularum. A: Bioassay and NMR spectra of fraction 75-50-A. B Bioassay and NMR spectra of fraction 75-50-E. C: Bioassay and NMR spectra of fraction 75-50-F.

D: Bioassay and NMR spectra of fraction 75-50-G. E: Bioassay and NMR spectra of fraction 75-50-H. F: Bioassay and NMR spectra of fraction 75-50-I. G: Bioassay and NMR spectra of fraction 75-50-K. H: Bioassay and NMR spectra of fraction 75-50-P. Bioassays was carried out using BY4741 WT cells, grown in three concentrations of the fractions dissolved in DMSO, and growth profiles of fraction treated cells were compared to the growth profile of the DMSO control $(0 \% \mathrm{v} / \mathrm{v}$ fraction). Red dashed lines indicate $80 \%$ residual growth. NMR spectra were obtained by dissolving sub samples of each pooled fraction in deuterated methanol, and spectroscopy was carried out using a Varian DirectDrive spectrometer, at $600 \mathrm{MHz}$ for ${ }^{1} \mathrm{H}$. 


\subsubsection{The 75-50\% LH20 1L bulk fractions are bioactive}

The two remaining fractions (at $1 \mathrm{~L}$ each) from the $\mathrm{LH} 20$ fractionation of the $75-50 \%$ fraction of the $P$. insularum aqueous extracts were hypothesized to contain the bioactive component of the extracts. This was assessed by bioassays including analyses for yeast growth in presence and absence of iron supplementation. Furthermore, NMR spectroscopic analyses were carried out on sub-samples of the fractions at $600 \mathrm{MHz}$ for ${ }^{1} \mathrm{H}$ using a Varian DirectDrive spectrometer. Fractions $\mathrm{Q}$ and R obtained from the LH20 size exclusion column chromatography of the $75-50 \%$ fraction of the aqueous extract of $P$. insularum were able to reduce yeast growth (Fig 5.9). Further, this growth inhibition was rescued with the addition of exogenous $\mathrm{FeCl}_{3}$ (Fig 5.9). Interestingly, the 75-50- $\mathrm{R}$ fraction was marginally more bioactive than the 75-50-Q fraction. The NMR spectra of both fractions displayed similar profiles (Fig 5.9). Of most importance is the presence of the broad peak resonating at 6-7 ppm (red dashed box). This broad peak was not present in the fractions tested in the previous experiment (Fig 5.8) and may indicate the area of the spectra corresponding to the compound or compounds exhibiting the iron chelation bioactivity of interest.
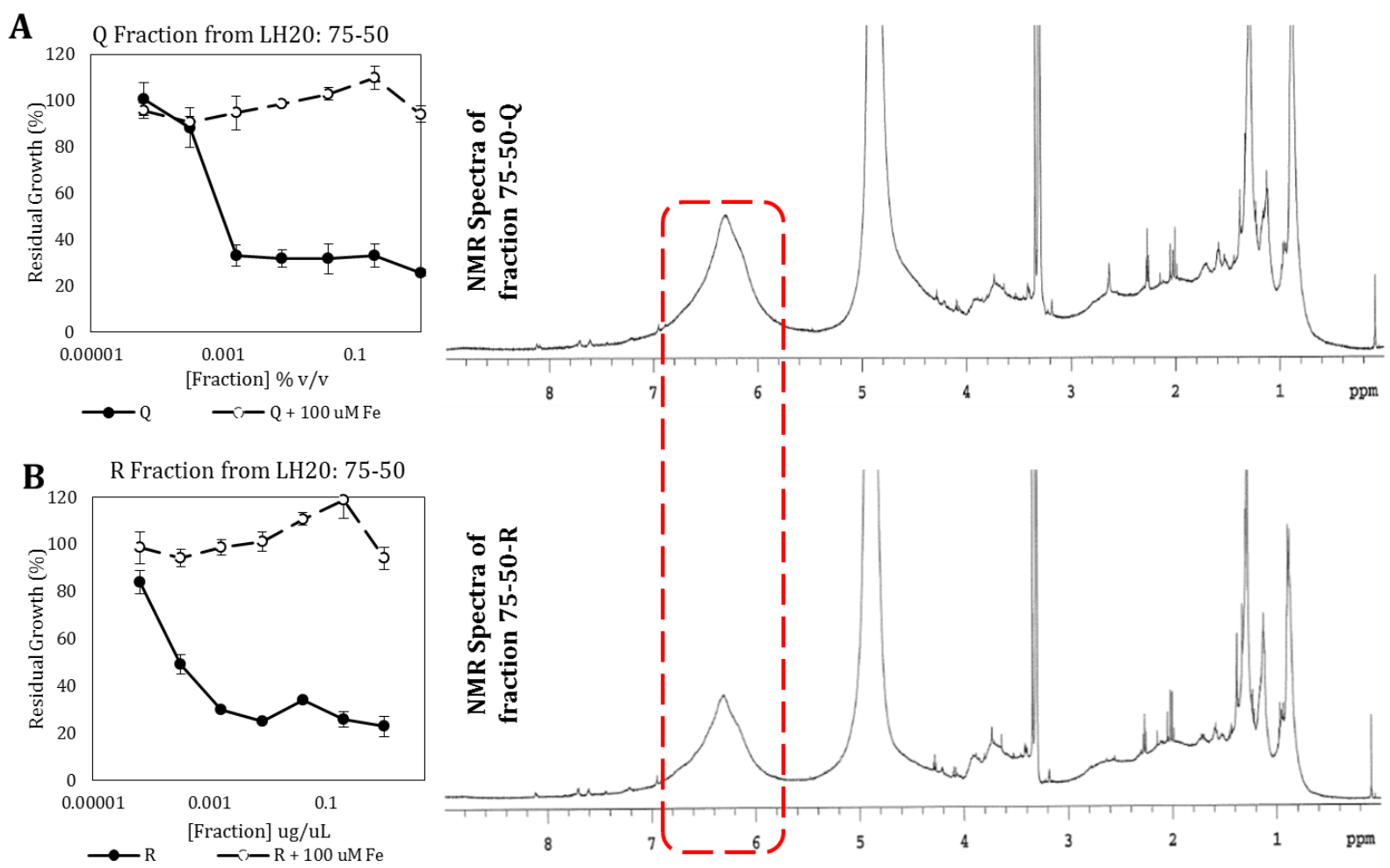
Figure 5.9: Bioassays and NMR spectra of the 75-50-Q and 75-50-R LH20 fractions. A: Bioassay and ${ }^{1} \mathrm{H}$ NMR spectrum of fraction 75-50-Q. B: Bioassay and ${ }^{1} \mathrm{H}$ NMR spectrum of fraction 75-50-R. Bioassays were carried out using BY4741 WT yeast strain, grown in the presence of increasing concentration of the fractions, ranging from $6.4 \times 10^{-5}$ to $1 \%$ observed in the A, E, F, G, H, I, K and P fractions.

5.3.9. Expression of Fet3p and Ftr1p iron transporters are upregulated in the presence of the selected bioactive fractions

The expression levels of the high-affinity iron transport system of yeast in the presence of the selected bioactive fractions were monitored as previously described in Chapter 3. Tracking the intensity of the green fluorescence resulting from the GFP tagged onto the protein of interest, we observed that Fet $3 p$ and Ftr1 $p$ forming the high-affinity iron transport system of yeast at the plasma membrane, were shown to be upregulated when yeast cells were treated with the aqueous extract of $P$. insularum (Fig 3.11). To determine if the fractions leading to fractions $Q$ and $\mathrm{R}$ elicited the same effect on the expression of iron transporters as the aqueous extract of $P$. insularum, Fet3p-GFP and Ftr1p-GFP yeast strains were grown in the presence of each of the fractions of interest (i.e., $75 \%$ fraction, $75-50 \%$ fraction, $75-50-Q$ and $75-50-R$ fractions) as well as iron free media and the iron chelator control (Fig 5.10). The crude extract was also included for comparative purposes. The intensity of green fluorescence observed from the GFP tagged onto Fet3p and Ftr1p were visually monitored, and we visually detected increased green fluorescence from GFP tagged onto both Fet3p (Fig 5.10A) and Ftr1p (Fig 5.10B) when treated with all the tested fractions, as well as the crude extract, compared to the control.

The expression profiles of Fet3p-GFP showed significantly increased expression levels in the presence of the crude aqueous extract as well as the fractions from the various steps of column chromatography (Fig 5.10A). While treatment of Fet3p-GFP with $0.05 \% \mathrm{v} / \mathrm{v}$ of the crude aqueous extract of $P$. insularum elicited a significant $30 \%$ increase in the expression of Fet $3 p$, treatment with $0.01 \% \mathrm{v} / \mathrm{v}$ of the $75 \% \mathrm{HP} 20$ fraction, or the $50 \%$ HP20ss fraction produced a significant $60 \%$ increase 
in the expression of Fet3p. Impressively, treatment with $0.01 \% \mathrm{v} / \mathrm{v}$ of the $\mathrm{Q}$ and $\mathrm{R}$ fractions from LH20 column chromatography led to increases of more than $100 \%$ in the expression of Fet $3 p$ which were statistically significant. Significant increases were also observed from Fet3p-GFP strains grown in iron free media and in the presence of the iron chelator by approximately $60 \%$ and $30 \%$, respectively. These findings indicate that fractions $75 \%$ and $75-50 \%$ induced an upregulation of Fet3p similar to that observed under iron-free conditions, while the crude aqueous extract elicited a response similar to that of the iron chelator control.

The expression of Ftr1p-GFP was also increased in the presence of the crude extract as well as the fractions, as observed from the higher intensity of the GFP signal from the extract treatments compared to the control (Fig 5.10B). Treatment with $0.05 \% \mathrm{v} / \mathrm{v}$ of the crude aqueous extract produced a significant approximate $30 \%$ increase in the expression of Ftr1p, whilst treatment with $0.01 \%$ of the fractions produced statistically significant increased expression ranging from $70-100 \%$. Growth in iron free media or in the presence of the iron chelator produced approximately $30 \%$ increased Ftr1p expression which were statistically significant. Together, the Fet $3 p$ and the Ftr1p results indicate that the step-wise fractionation carried out did not compromise the activity of the chemical components of interest, and that the fractions isolated with bioactivity retained the ironmediated activity of interest. 


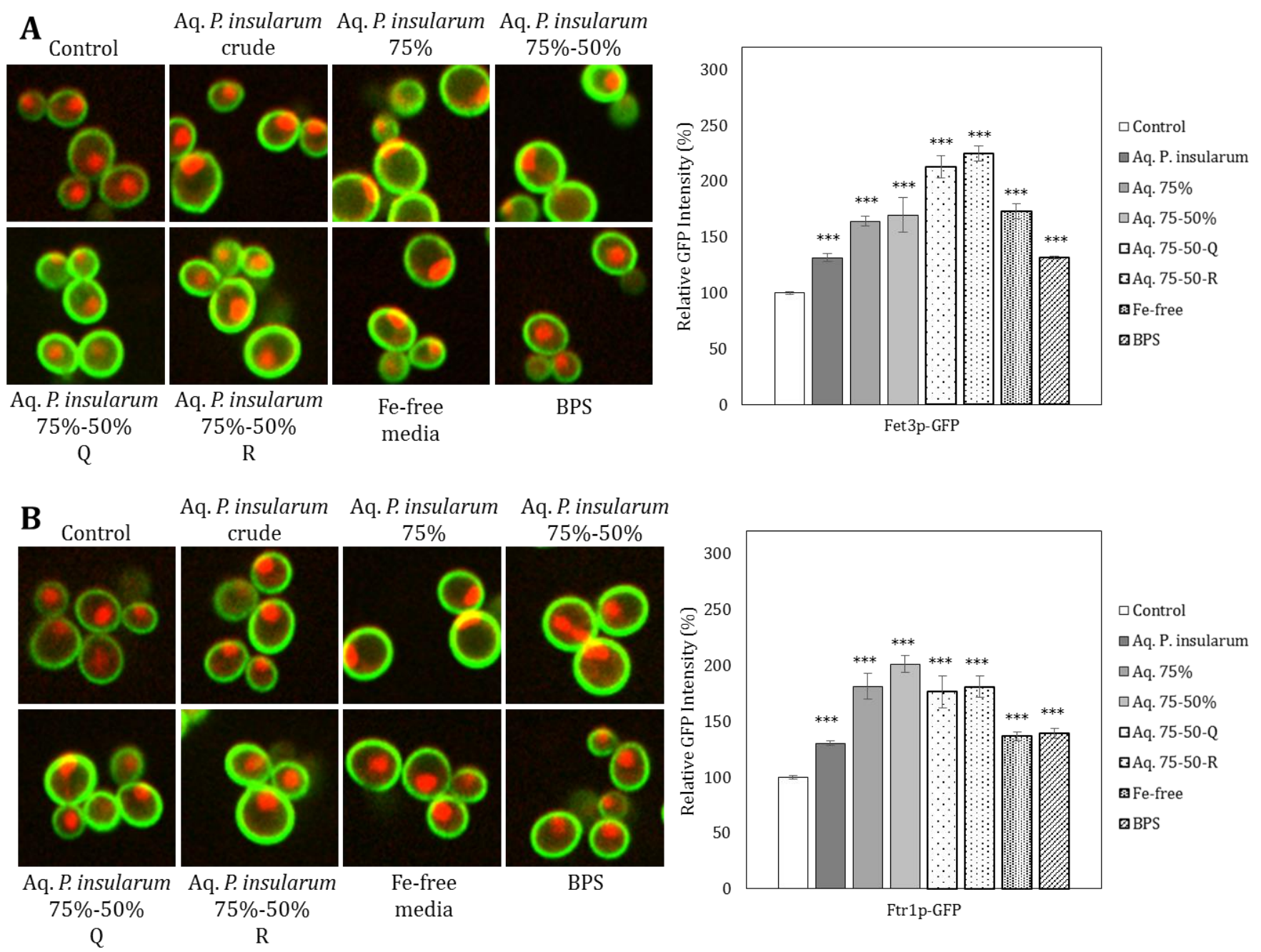

Figure 5.10: Effects of the fractions leading to partially purified LH-20 bioactive fractions on the expression of the high affinity iron transporters Fet3p and Ftr1p. A: GFP profile of Fet3p-GFP across the 8 treatments tested, including control, $0.05 \% \mathrm{v} / \mathrm{v}$ of the crude aqueous extract of $P$. insularum extract (Aq. P. insularum crude), $0.01 \% \mathrm{v} / \mathrm{v}$ of the $75 \%$ HP20 fraction from the aqueous extract (Aq. $P$. insularum 75\%), $0.01 \% \mathrm{v} / \mathrm{v}$ of the 50\% HP20ss fraction from the 75\% HP20 fraction (Aq. P. insularum), $0.01 \% \mathrm{v} / \mathrm{v}$ of Q and R fractions from the LH20 fractionation of 75-50\% fractions (Aq. P. insularum 75\%50\% Q and Aq. P. insularum 75\%-50\% R), and Fet3p-GFP grown in iron free media (Fe-free media) and in the presence of BPS. B: GFP profile of Ftr1p-GFP across the 8 treatments tested. Microscopy images were obtained using a Perkin Elmer Evotec spinning disc Opera confocal microscope. Quantification shown in the bar-graphs was obtained using Acapella software. To determine if the increase in GFP intensity observed was significant, statistical analyses were carried out using Microsoft Excel paired two sample for means, one tailed t-test. (*** $=$ p-value $<0.001)$. 


\subsubsection{Fraction R from LH20 column chromatography is a condensed tannin}

The NMR profiles obtained from the aqueous extracts of $P$. insularum are characteristic of tannins (Lavoie et al., 2015). The tannin assay employs ferric chloride, which leads to the formation of tannin-iron complexes that are brownish-green or blue-black in colour (Dhandapani \& Sabna, 2008) was used to determine if fraction $R$ contained tannins. Indeed, when assayed against this simple colorimetric test, fraction $\mathrm{R}$ produced the blue-black colour indicative of the presence of tannins in fraction R (Fig 5.11A). Tannins are polyphenolic secondary metabolites that are widely distributed in plants. The three classes of tannins include hydrolysable tannins, condensed tannins and complex tannins (Cos et al., 2003). As NMR analyses of fraction R showed that it did not contain the typical building blocks of hydrolysable tannins (data not shown), we sought to determine if the tannin is a condensed tannin. An easy colorimetric assay that can distinguish condensed tannin from the others is the acid-butanol assay (Fig 5.11B), which results in the hydrolysis of the condensed tannin and formation of red anthocyanidin precipitate (Schofield et al., 2001). To determine if fraction $\mathrm{R}$ comprises condensed tannins, it was exposed to the acid-butanol test, which resulted in the formation of the positive reaction and the red precipitate (Fig 5.11C). However, despite these promising findings, due to the time limitation of this work, we were not able to continue with further purification and identification efforts.
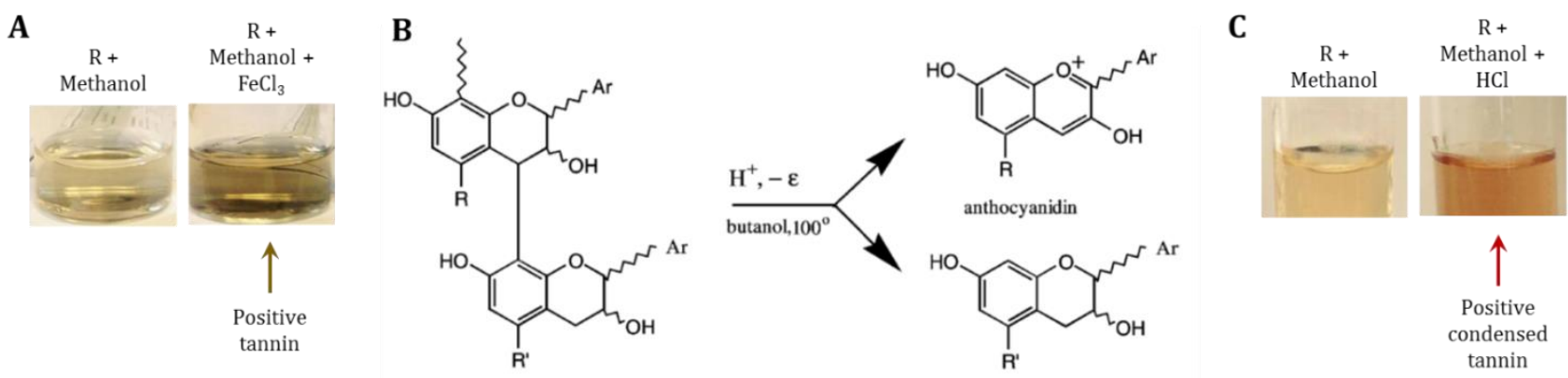

Figure 5.11: Tannin and condensed tannin tests. A: Tannin test of the R fraction from LH20 column chromatography. The ferric chloride test indicates positive for tannins with a brownish-green/blue-black colouring (brown arrow). B: The chemical basis of the acid-butanol condensed tannin test. The reaction involves oxidation of the condensed tannin and the formation of the red coloured anthocyanidin unit (Schofield et al., 2001). C: Condensed tannin test of fraction R from the LH20 column chromatography. The red precipitate is indicative of a positive results is highlighted by red arrow. 


\subsection{Discussion}

The aim of this chapter was to identify the compound responsible for the iron chelation activity that was determined to be the mechanism of action of the $P$. insularum extracts investigated in Chapter 3. The findings that have been presented herein traced the iron chelation activity of the aqueous extract through several column chromatography fractionations to a compound or compounds with ${ }^{1} \mathrm{H}$ NMR resonance at 6-7 ppm indicative of condensed tannins (Fig 5.12).

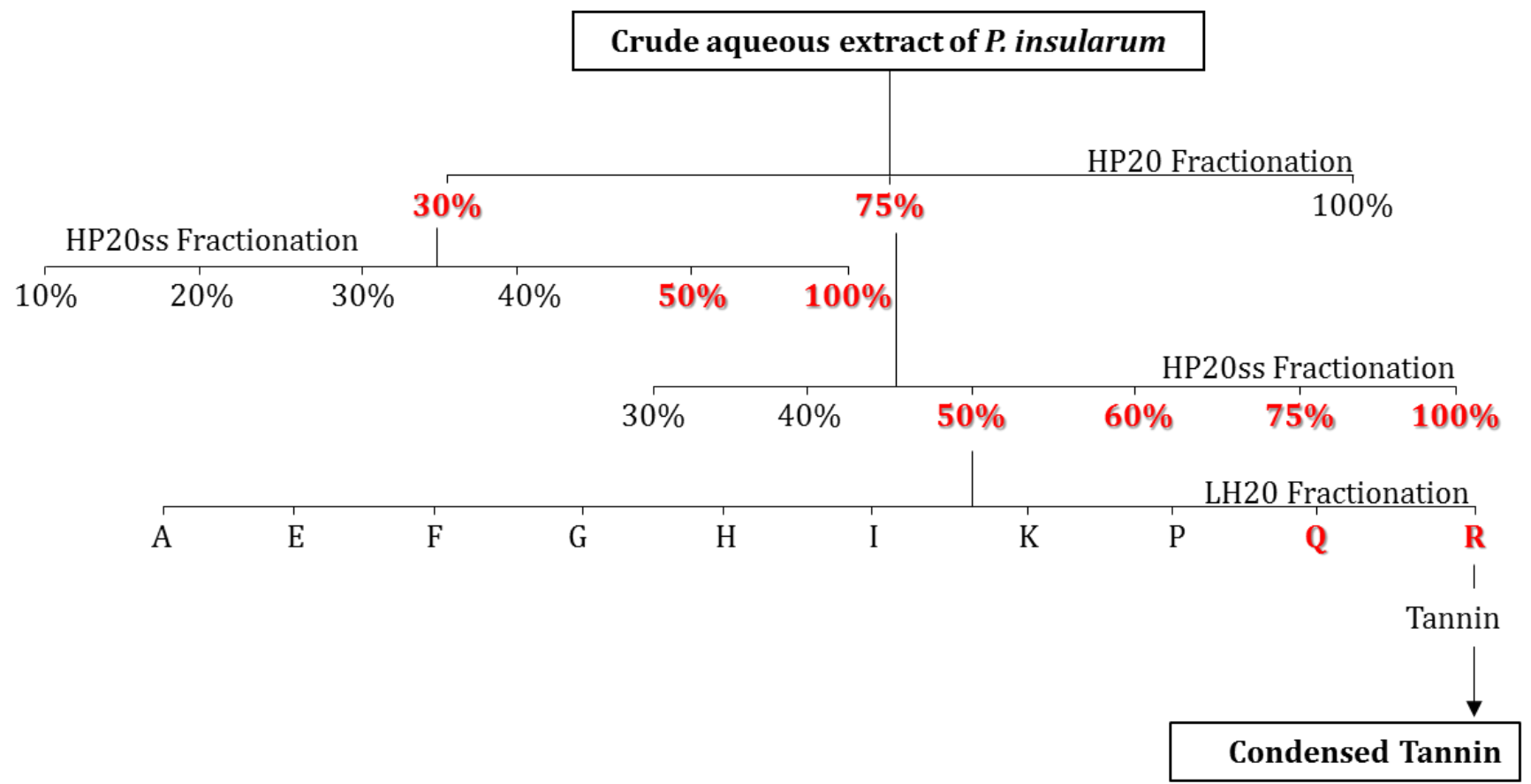

Figure 5.12: Summary of the bioassay guided fractionation of the crude aqueous extract of $\boldsymbol{P}$. insularum. HP20 fractionation produced $30 \%$ and $75 \%$ fractions that were bioactive (in red). The 30\% fraction was further fractionated with an HP20ss column, producing bioactive (in red) 50\% and 100\% fractions. HP20ss fractionation of the 75\% HP20 fraction produced bioactive (in red) fractions $50 \%$ and $60-100 \%$ which were pooled. The $50 \%$ fraction from the HP20ss fractionation of the $75 \%$ was further fractionated with an LH20 column, producing 10 fractions, from which only Q and R were shown to be bioactive (in red). Fraction $\mathrm{R}$ tested positive for tannin and condensed tannin.

The fractionation scheme utilized in the first two fractionation steps here were on the basis of polarity of extract components (Fig 4.13). Essentially, most of the components of the extract elute at higher polarity elution solvent (i.e., low acetone concentration) and are often composed of polysaccharides, sugars, soluble proteins and nucleotides. At lower polarity elution concentrations (i.e., high acetone concentration), lipids and fatty acids are eluted. The area highlighted (red dashed 
box in Fig 4.13) is often the area of most interest because this is the polarity range where secondary metabolites elute that are often responsible for bioactivity of small molecules. Interestingly, detecting the iron chelation bioactivity from the original 30\% and 75\% acetone HP20 fractions, and later from the $50 \%$ acetone HP20ss fraction, correlated to the area of the basic concept of polarity fractionation curve of natural products where plant secondary metabolites often elute. This suggested the compound responsible for the activity of interest is a plant secondary metabolite.

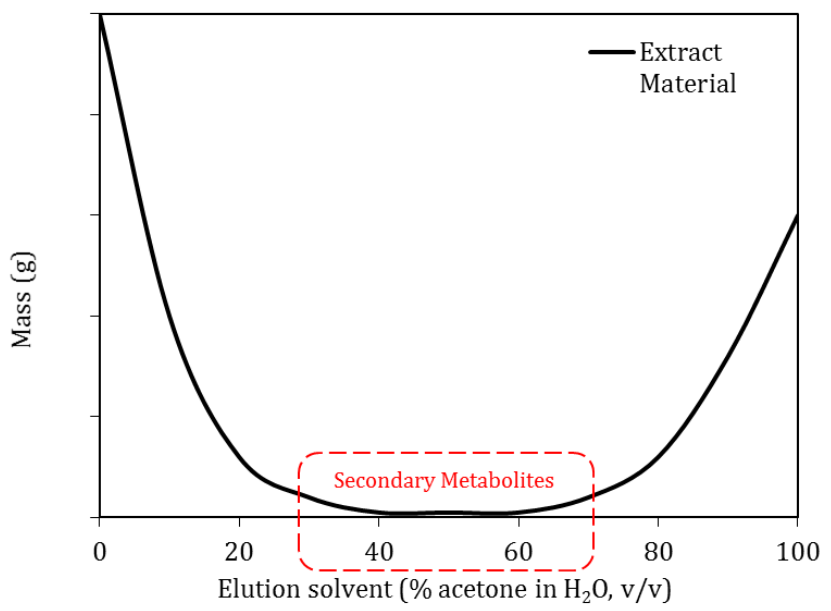

Figure 5.13: Basic concept of polar fractionation of crude extract material. Polysaccharides and sugars elute at higher polarity (i.e., low acetone concentrations) while lipids elute at lower polarity (i.e., high acetone concentrations). The area highlighted by the red dashed box is the area of most interest where secondary metabolites elute, around $30-70 \%$ acetone in water, v/v (West, 2001).

LH20 column chromatography employing separation on a size exclusion basis separated components based on size, where larger molecules pass through the columns more quickly than the smaller molecules. The lack of bioactivity from the originally collected $5 \mathrm{~mL}$ fractions (330 fractions) indicated that the larger molecules and subsequently smaller molecules that were eluted during these fractions were not responsible for the bioactivity. Further, it suggested that the molecular entity responsible for the iron chelation bioactivity of interest was one that was problematic to elute off an LH20 column with methanol most likely due to the strong adsorption of aromatic (benzene) components to the resin surfaces. This is in line with tannins, which often require a combination of acetone or alcohol in water for their elution off an LH20 column (Dai \& Mumper, 2010). 
Our bioassay guided fractionation approach required a well characterized bioassay probing a biological activity of interest. Given our previous findings, we employed two assays: the yeast growth inhibition assay, and the iron rescue assay. As such, our approach allowed the tracking of the iron chelating bioactivity of interest throughout the different fractionation steps. However, this approach is also disadvantageous; because of its inherent pursuit of a specific bioactivity, this approach ignores novel compounds that do not exhibit the activity of interest. Further, in instances where bioactivity is a consequence of the synergistic activities of two or more compounds, fractionation leads to the loss of activity. Additionally, this approach often leads to the isolation of already known compounds, although this can be rectified, as was carried out here, through the incorporation of spectroscopic analyses to prevent further fractionation where an already known compound is identified as the bioactive component.

Tannins are a type of secondary metabolite that belong to the phenolics group, and have been widely reported to have antimicrobial and anti-inflammatory properties used in medicinal application as diuretics, astringents and antiseptics (Kabera et al., 2014; Khanbabaee \& van Ree, 2001). Mechanisms by which tannins exert their effects include enzyme inhibition and substrate deprivation, activity on cellular membranes and metal complexation (Scalbert, 1991). Indeed, iron chelation has been reported from various tannins including tannic acid, procyanidin and catechins (Hagerman et al., 1998; South \& Miller, 1998). The presence of condensed tannins in our final fraction producing the iron chelation activity of interest is therefore consistent with the long history behind the metal deprivation activity of tannins (Scalbert, 1991). It is also of interest given the positive result obtained from our iron chelation assay (Fig 3.15) meaning that it is likely that another plant extract possessing iron-chelating tannins would have produced a similar positive result from this CAS assay. Also of interest and requiring consideration is the ability of tannins to precipitate proteins. This is of interest because bioactivities depending on proteins could be confounded by this activity of tannins. However, this cannot be the case here, because we have demonstrated that iron chelation carried out in a non-cell lysate environment (i.e., in chemical solutions only with the 
extract) exhibited iron chelation, indicating that iron chelation of $P$. insularum is independent of proteins, and therefore cannot be attributed to the protein precipitation activity of tannins.

Condensed tannins have a wide array of therapeutic functions in mammalian models of various human diseases. For instance, condensed tannins isolated from cacao inhibited the development of cataracts in diabetic rats (Osakabe et al., 2003). Additionally, condensed tannins from grapeseed protected cardiomyocites from ischemia, reduced cardiotoxicity resulting from Doxorubicin treatment, and reduced angiogenesis resulting from colon cancer (Huang et al., 2012; Li et al., 2010; Shao et al., 2009). Other biological activities that have been observed from condensed tannins include antimicrobial (Avorn et al., 1994; de Bryune et al., 1999; Howell et al., 2002; Kolodziej et al., 1999; Kontiokari et a., 1999), antiviral (Barnard et al., 1993; de Bruyne et al., 1999; Ma et al., 2000; Shahat et al., 2002), and antiprotozoal activities (Calzada et al., 1999; Calzada et al., 2000; Kolodziej et al., 2001). Antioxidant effects of condensed tannins have also been studied and attributed to their ability to scavenge radicals (Nanjo et al., 1996; Plumb et al., 1993; Yokozawa et al., 1998), inhibit pro-oxidant enzymes such as lipoxygenase (Moini et al., 2002; Schewe et al., 2001; Schewe et al., 2002) and chelate metals (Facino et al., 2002; Yoneda \& Nakatsubo, 1998). Antiinflammatory effects of condensed tannins have also been investigated and proposed to be mediated through the NF-KB pathway (Park et al., 2000; Saliou et al., 2001; Yang et al., 1998), which is the same pathway proposed herein to mediate the iron chelating anti-inflammatory activities demonstrated by the aqueous extract of $P$. insularum.

Up to this point, we have not considered the possibility of multiple bioactive compounds in the extracts from P. insularum. The findings from our LH2O bioassay guided fractionation however showed that bioactivity was completely absent from fractions without the 6-7 ppm hump (Fig 5.8). These findings support the premise that bioactivity is restricted to the unresolved 6-7 ppm hump. However, the possibility that the unresolved hump may contain more than one compound still suggests that there may be more bioactive compounds, although this will be further clarified upon further purification of the unresolved hump or the R fraction. 
In summary, we have identified condensed tannins as the chemical group of secondary metabolites that mediate the iron-chelating bioactivity of $P$. insularum extracts in yeast and mammalian cells. However, the complex nature of the condensed tannin isolated from the aqueous extract of $P$. insularum is currently unknown and requires further investigation to identify the condensed tannin. Furthermore, the applicability of the condensed tannin isolated herein as a lead compound needs to be investigated, where for instance, modifications to its structure which is yet to be determined, may improve its activity and drug potential. 


\section{Chapter 6:}

\section{Implications \& Future Directions}

\subsection{Summary}

The overall aim of this project was to ascertain the pharmaceutical potential of traditional Samoan medicinal plants via determining their mechanism of action and their chemical composition. In Chapter 2, the bioactivity of Samoan medicinal plants was assessed from which 15 of 22 extracts were shown to be bioactive in yeast. More significantly, 10 of 11 plants produced an extract that reduced yeast growth to an extent amenable to genome-wide analyses to identify gene deletions that significantly enhance bioactivity. Extracts from the medicinal plant Pscyhotria insularum were identified as the most potent against yeast growth. In Chapter 3, iron chelation was established as the mechanism of action for the extracts from P. insularum. This iron chelation activity was also shown to mediate the anti-inflammatory effects of the extracts in murine macrophages. In Chapter 4, it was further determined that the iron chelation mechanism does not include targeting a single gene, a result consistent with the extracts targeting iron directly. In Chapter 5, bioassay-guided fractionations were used to determine that condensed tannins were the class of compounds responsible for the iron chelation activity of the extract. In this chapter, the implications of the findings presented thus far will be discussed.

\subsection{Implications on P. insularum as a potential pharmaceutical}

Leaf extracts of the Samoan medicinal plant $P$. insularum have been used since the 1800 's to treat supernaturally induced ailments, abdominal distress and ailments associated with inflammation (e.g., wounds, infections, fevers) (Cox et al., 1989; Whistler, 1996). Various studies have demonstrated extracts from $P$. insularum reduced hypertension, motor function, ear oedema as well as prostaglandin synthesis, and were toxic at high concentrations in mice (Cox et al., 1989; 
Dunstan et al., 1997; Norton et al., 1974). However, the underlying molecular mechanism by which P. insularum extracts mediated these different effects was not investigated. In this thesis, the iron chelation mechanism of action of the $P$. insularum extracts was identified, and in combination with available literature, can account for these activities. For instance, it has been demonstrated that iron chelation promoted vasodilation and subsequently lowered blood pressure (Duffy et al., 2001), thus providing a tentative connection between the iron chelation mechanism of action identified in this dissertation and the hypotensive activity of $P$. insularum (Norton et al., 1974). Additionally, the work described herein proposed a NF-KB dependent link between iron chelation and the antiinflammatory response detected in murine macrophages treated with $P$. insularum extracts. Indeed, this link corroborates the previous report that $P$. insularum reduced prostaglandin biosynthesis (Dunstan et al., 1997), since cyclooxygenase-2 (COX-2) that regulates prostaglandin synthesis is in turn regulated by NF-KB (Inoue \& Tanabe, 1998, Newton et al., 1997; Schmedtje et al., 1997). Therefore, a reduction in NF-KB activation caused by iron chelation would lead to reduced COX-2 activity and prostaglandin synthesis. In contrast, work by Tanji et al. (2001) on the iron chelator desferroxamine (DFX) demonstrated that DFX upregulated the production of prostaglandin, which promoted the inflammatory process. The discrepancy between the results of the iron chelator DFX and the iron chelation activity of $P$. insularum may be a consequence of the different cells used, as the work described herein utilized primary murine macrophages, while Tanji et al. (2001) used the immortalized U937 human macrophage cell line.

Although elucidating the MOA of the $P$. insularum leaf extracts is a novel contribution of this thesis to the fields of genetics and molecular biology, identification of the class of bioactive compounds responsible for the iron chelating activity as condensed tannins is also a noteworthy outcome of this thesis. Condensed tannins have a wide array of therapeutic functions in mammalian models such as inhibiting the development of cataracts in diabetic rats (Osakabe et al., 2003); protection of cardiomyocites from ischemia, reduced cardiotoxicity resulting from Doxorubicin treatment, and reduced angiogenesis resulting from colon cancer (Huang et al., 2012; Li et al., 2010; 
Shao et al., 2009); as well as antimicrobial (Avorn et al., 1994; de Bryune et al., 1999; Howell et al., 2002; Kolodziej et al., 1999; Kontiokari et a., 1999), antiviral (Barnard et al., 1993; de Bruyne et al., 1999; Ma et al., 2000; Shahat et al., 2002), and antiprotozoal activities (Calzada et al., 1999; Calzada et al., 2000; Kolodziej et al., 2001). Condensed tannins have also been to chelate metals (Facino et al., 2002; Yoneda \& Nakatsubo, 1998) and have anti-inflammatory properties (Park et al., 2000; Saliou et al., 2001; Yang et al., 1998).

Iron chelators are largely becoming of great interest as drug candidates in regulating diseases exhibiting iron overload. Primarily, excess iron in the body often arises from a genetic condition (e.g., hereditary haemochromatosis), but can also result from chronic blood transfusion (e.g., to treat $\beta$-thalassemia major patients) (Faa \& Crispon, 1999). In addition, iron deposits accumulate to toxic levels in neurodegenerative disorders such as Alzheimer's disease (Connor et al., 1992), Parkinson's disease (Sofic et al., 1988), Huntington's disease (Dexter et al., 1991) and HIV encephalopathy (Miszkiel et al., 1997). While DFX has been the iron chelator of choice for the treatment of hereditary haemochromatosis and $\beta$-thalassemia major patients, it requires extended subcutaneous infusion because it has a short half-life and is inactive when administered orally (Aouad et al., 2002). Deferiprone (DFP) is another iron chelator that is approved for use in the EU, Canada and the US particularly in $\beta$-thalassemia major patients who do not respond to other iron chelation therapy (Poggiali et al., 2012). Interestingly, analogues of DFP have been investigated for potentially treating neurodegenerative diseases (Molina-Holgado et al., 2008). Deferasirox (DFS) is the most orally-administered iron chelator approved and can be used in patients as young as two years old (Piga et al., 2006). However, despite these successes in the development of iron chelators as therapeutic agents, these drugs elicit toxic adverse effects such as growth retardation, proteinuria, elevated liver enzymes and allergic reactions (Poggiali et al., 2012). This highlights the need for a new iron chelator in the clinic, which ideally should be able to cross the gastro-intestinal tract, be selective for iron and have low toxicity but should not be able to cross the blood-placenta 
barrier for the treatment of general iron imbalance; in the cases of neurodegenerative diseases, the iron chelator should also be able to cross the blood-brain barrier (Faa \& Crisponi, 1999).

Condensed tannins have demonstrated great potential as drug candidates given primary beneficial effects on basic cellular processes such as oxidative stress and secondary health effects on complications arising from diseases. By extension, this makes the condensed tannin isolated from the aqueous extract of $P$. insularum a potential drug candidate, made even more convincing given its applicability as an iron chelator. However, the applicability of compounds such as condensed tannins as iron chelators in modern day medicine has not yet been investigated. Indeed, there is contradicting literature regarding the uptake of condensed tannins from the intestinal environment into the bloodstream and given the size complexity of condensed tannins, this may be a hindrance. Further investigation is warranted into identifying the compositional complexity of the condensed tannin and establishing a connection between iron chelation arising from $P$. insularum extracts and the proposed downregulation of NF-KB manifesting in the anti-inflammatory effects of the $P$. insularum extracts.

\subsection{Implications on P. insularum application in Samoan medicine}

While the demonstrated anti-inflammatory effects of $P$. insularum extracts are in line with the application of the plant to treat inflammation-related ailments in Samoa, this research has also brought to light that $P$. insularum may treat additional diseases given its iron chelating activity and the relevance of iron chelating agents to diseases with iron imbalance. As mentioned previously, hereditary haemochromatosis is a genetic condition that manifests in iron overload (Faa \& Crisponi, 1999). From a sample of 169 Polynesian individuals, there was a $0.89 \%$ prevalence of a mutation that results in this condition (Cullen et al., 1998). By extrapolation, this suggests that of the whole population of Samoa ( 200,000 people), approximately 1,780 people have haemochromatosis specific to this mutation, not including haemochromatosis of other mutations. Alzheimer's disease, which has increasing evidence of iron imbalance (Connor et al., 1992), recorded a move up in 
ranking for the cause of premature death in Samoa, from $56^{\text {th }}$ in 1990 to $25^{\text {th }}$ in 2010 ("GBD Profile: Samoa", 2010), indicative of increasing prevalence in Samoa. Overall, there is increasing prevalence of diseases including disrupted iron homeostasis in Samoa, and there is no better place to turn than to one of Samoa's own traditional medicinal plants.

\subsection{Implications on the P. insularum plant}

Whistler (1986) reported the Psychotria genus of the Rubiaceae family as one of the most diverse genera across the Pacific, with the Samoan archipelago home to 20 Pscyhotria species. Although this genus was reported as abundant in 1986, a more recent report of its abundance, or more specifically that of $P$. insularum, is not available. To that end, the medicinal plant $P$. insularum has not yet been assessed by the International Union for Conservation of Nature (IUCN) for their list of endangered and threatened plant species (www.iucnredlist.org). When plants were being collected for this study, P. insularum was not particularly abundant in national parks and had to be sourced from a single private property owned by local healers actively cultivating the species. Barring the possibility of this was not due to environmental factors (e.g., seasonal fluctuation) this suggests that the abundance of the species in the wild is reduced. Therefore, the wild populations of P. insularum need to be evaluated particularly to avoid the future scenario where pharmaceutical potential is realized but wild populations are endangered.

This study utilized a single source of $P$. insularum from a private property of relatively low elevation (40-50 m). The study cannot however report on the bioactivity of leaves obtained from $P$. insularum plants at higher elevations or from different locations. Indeed, different populations of the Samoan medicinal plant Homalanthus nutans contained different amounts of the anti-HIV compound prostratin (Johnson et al., 2008). Therefore, to broaden the applicability and the validity of the findings from this project, it is important to perform similar bioactivity studies on extracts from additional $P$. insularum plants. We recommend that yeast growth assays, iron rescue assays and NMRs be conducted to determine if the iron chelating activity of condensed tannins that we 
identified in P. insularum extracts from one location (Chapters $3 \& 4$ ) are also active in new extracts from additional locations and elevations in Samoa. In addition, it would be interesting to quantify the iron-chelating potency of condensed tannins in plant parts other than $P$. insularum leaves, particularly since different parts of $H$. nutans plant contained varying amounts of prostratin (Johnson et al., 2008).

\subsection{Implications on natural products in Samoa}

Previous work demonstrated the great potential of Samoan medicinal plants, illustrating bioactivity in animal and human cells, albeit with minimal knowledge on the underlying molecular mechanisms mediating their bioactivities (Cox et al., 1989; Dunstan et al., 1997; Norton et al., 1974). This thesis on $P$. insularum has produced results that further corroborates the pharmaceutical potential of Samoan medicinal plants via the determination of the mechanism of action of $P$. insularum leaf extracts, and thus warrants extension of this research into under-studied Samoan medicinal plants. Perhaps it may also warrant an investigation into other Samoan resources as sources of natural products as a whole (e.g., marine products). A Samoan saying goes "E fofo ele alamea le alamea", which translates into "An injury from a crown-of-thorns starfish will be cured by the crown-of-thorns starfish" because the crown-of-thorns starfish (Acanthaster planci), when placed onto the injury site, will suck the toxin out itself. Indeed, bioactive compounds have been isolated from the toxins of $A$. planci that induce apoptosis, oxidative stress and ER stress (Lee et al., 2014; Lee et al., 2015). These findings reiterate that there is traditional knowledge to be exploited in Samoa regarding native plants and marine organisms, specifically with realizing the pharmaceutical potential of native natural products isolated from these sources.

\subsection{Future Experiments}

In Chapter 3, we identified a link between iron chelation and inflammation work, and had proposed a NF-KB mediated connection. This relationship requires further investigation, potentially 
by monitoring kinase activities of inhibitors of NF- $\mathrm{KB}$ in the presence of the extract, control treatment, as well as in high iron conditions. It is postulated that the kinase activity of IKK (inhibitor of NF-KB kinase) will be reduced in the presence of the $P$. insularum, preventing the inhibitors of NF$\mathrm{KB}$ dissociating from NF-KB and ultimating inhibiting the downregulation of IL-10, thereby resulting in an anti-inflammatory response. In Chapter 4, it was determined that a similarity between the diploid pooled genome-wide analyses of the extracts of $P$. insularum and the agar-based haploid screen was the absence of iron transporters and their transcription factor from hypersensitive mutants. To confirm that this was a caveat of the assay as observed in agar, we propose a validation of diploid iron transporter mutants individually against the extracts of $P$. insularum. Given that the haploid strains were hypersensitive in liquid, we expect the diploid strains will also be hypersensitive, and that the lack of detection from the pooled assays may be a consequence of high-throughput screening. Alternatively, it is also possible that in a pooled environment, there is an environment of multiple strains that may survive less well than iron-dependent mutants that were thus killed, and their waste material recycled including iron, leading to better growth of iron-related mutants. We were not able to fully isolate the bioactive component of the $P$. insularum extracts in our efforts described in Chapter 5, and we were only able to partially purify the crude extract and determine that the bioactivity was attributable to a condensed tannin. We propose further chemical analyses utilizing HPLC in combination with mass spectrometry may isolate the compound and permit its identification.

Given the potential of iron chelators to mediate diseases with aberrant iron regulation, there is a possibility our iron chelating condensed tannin may be beneficial in such diseases. For instance, iron dysregulation has been reported in diseases such as Alzheimer's, Parkinson's, obesity and diabetes. We propose that disease models for these be treated with varying concentrations of our condensed tannin to investigate whether our condensed tannin can alleviate some of these disease symptoms. Furthermore, it would be interesting to survey the prevalence of $P$. insularum use in traditional Samoan medicine, and its reported efficacy and whether the reported efficacy 
correlated to the anti-inflammatory activity or iron chelating activity of $P$. insularum extracts as determined from this dissertation.

\subsection{Conclusion}

In conclusion, this dissertation used genome-wide analyses to establish the MOA of crude extracts of a medicinal plant. We established that the leaf extracts of the Samoan medicinal plant $P$. insularum mediated its bioactivity via an iron chelating mechanism. We also determined that the bioactive component of the $P$. insularum extracts mediating this activity belonged to the chemical class of condensed tannins. Indeed, findings from this work have made novel contributions to the broad fields of biology and chemistry, with multiple implications not only on the potential of this particular Samoan medicinal plant as a pharmaceutical agent, but also on its application in traditional Samoan medicine. Overall, these findings provide a strong and positive outlook for the potential for further discoveries to be made from Samoan medicinal plants. 


\section{References:}

Abe, F. and Hiraki, T. (2009). Mechanistic role of ergosterol in membrane rigidity and cycloheximide resistance in Saccharomyces cerevisiae. Biochimica et Biophysica Acta. 1788(3): 743-752

Aerts, AM.; Fracois, I.; Cammue, BPA. and Thevissen, K. (2008) The mode of antifungal action of plant, insect and human defensins. Cellular and Molecular Life Sciences 65: 2069-2079

Agne, B.; Meindl, NM.; Niderhoff, K.; Einwachter, H.; Rehling, P.; Sickmann, A.; Meyer, JE.; Girzalsky, W. and Kunau, WH. (2003). Pex8p: an intraperoxisomal organizer of the peroxisomal import machinery. Molecular Cell. 11(3): 635-646

Alanis-Garza, BA.; Gonzales-Gonzales, GM.; Salazar-Aranda, R.; Waksman de Torres, N. and RivasGalindo, VM. (2007). Screening of antifungal activity of plants from the northeast of Mexico. Journal of Ethnopharmacology. 114(3): 468-471

Alexander, DB. and Zuberer, DA. (1991). Use of chrome azurol S reagents to evaluate siderophore production by rhizosphere bacteria. Biology and Fertility of Soils. 12: 39-45

Ali-Shtayeh, MS. and Abu Ghdeib, SI. (1999). Antifungal activityof plant extracts against dermatophytes. Mycoses 41(5-6): 243-248

Alves, RRN. and Albuquerque, UP. (2013). Animals as a source of drugs: bioprospectiing and biodiversity conservation. In Alves, RRN and Rosa, IL (eds). Animals in Traditional Folk Medicine. Springer-Verlag Berlin Heidelberg. pp 67-89

Amerik, AY.; Nowak, J.; Swaminathan, S. and Hochstrasser, M. (2000). The Doa4 deubiquitinating enzyme is functionally linked to the vacuolar protein-sorting and endocytic pathways. Molecular Biology of the Cell. 11(10): 3365-3380

Aouad, F.; Florence, A.; Zhang, Y.; Collins, F.; Henry, C.; Ward, RJ. and Crichton, RR. (2002). Evaluation of new iron chelators and their therapeutic potential. Inorganic Chimica Acta. 339: $470-480$

Archin, NN. and Margolis, DM. (2014). Emerging strategies to deplete the HIV reservoir. Current Opinion in Infectious Diseases. 27(1): 29-35

Aronson, JK. (1977). Medicine in Staffodshire. British Medical Journal 16(1): 1031-1032 
Arthington, BA.; Hoskins, J.; Skatrud, PL. and Bard, M. (1991). Nucleotide sequence of the gene encoding yeast C-8 sterol isomerase. Gene. 107(1): 173-174

Askwith, C.; Eide, D.; van Ho, A.; Bernard, PS.; Li, L.; Davis-Kaplan, S.; Sipe, DM.; Kaplan, J. (1994). The FET3 gene of $S$. cerevisiae encodes a multicopper oxidase required for ferrous iron uptake. Cell. 76(2): 403-410

Atheron, J. and Jefferies, B. (2012). Rapid biodiversity assessment of upland Savaii, Samoa. Apia, Samoa: Ministry of Natural Resources \& Environment, Secretariat of the Pacific Regional Environment Programme (SPREP), retrieved from www.cepf.net/SiteCollectionDocuments/poly micro/59745 TechnicalReport RAP Upland Savaii.pdf

Amberg, DC.; Burke, DJ. and Strathern, JN. (2005). Methods in Yeast Genetics: A Cold Spring Harbor Laboratory Course Manual. New York: Cold Spring Harbor Lab Press

Avorn, J.; Monane, M.; Gurwitz, JH.; Glynn, RJ.; Choodnovskiy, I.; Lipsitz, LA. (1994). Reduction of bacteriuria and pyuria after ingestion of cranberry juice. JAMA. 27(10): 751-753

Azad, K.; Hossain, F. and Halim, MA. (2013). Screening of cellulose, pectinase and xylanase activities and optimization of radial mycelia growth of two thermophilic fungi. Bangladesh Journal of Botany. 42: 207-213

Baetz, K.; McHardy, L.; Gable, K.; Tarling, T.; Reberioux, D.; Bryan, J.; Andersen, RJ.; Dunn, T.; Hieter, P. and Roberge, M. (2004). Yeast genome-wide drug-induced haploinsufficiency screen to determine drug mode of action. Proceedings of the National Academy of Science 101(13): $4525-4530$

Baker, RT.; Tobias, JW. and Varshavsky, A. (1992). Ubiquitin-specific proteases of Saccharomyces cerevisiae. Cloning of UBP2 and UBP3, and functional analysis of the UBP gene family. Journal of Biological Chemistry. 267(32): 23364-23375

Baker, HM.; Anderson, BF. and Baker, EN. (2003). Dealing with iron: common structural principles in proteins that transport iron and heme. Proceedings of the National Academy of Science 100: $3579-3583$

Barnard, DL.; Smee, DF.; Huffman, JH.; Meyerson, LR. and Sidwell, RW. (1993). Antiherpes virus activity and mode of action of SP-303, a novel plant flavonoid. Chemotherapy. 39(3): 203211

Beh, CT. and Rine, J. (2004). A role for yeast oxysterol-binding protein homologs in endocytosis and in the maintenance of intracellular sterol-lipid distribution. Journal of Cell Science. 15: 29842996 
Bender, A. and Pringle, JR. (1991). Use of a screen for synthetic lethal and multicopy suppressee mutants to identify two new genes involved in morphogenesis in Saccharomyces cerevisiae. Molecular and Cellular Biology 11(3): 1295-1305

Benjamin, D.; Colombi, M.; Moroni, C. and Hall, MN. (2011). Rapamycin passes the torch: a new generation f mTOR inhibitors. Nature Reviews: Drug Discovery. 10(11): 868-880

Best, HA.; Matthews, JH.; Heathcott, RW.; Hanna, R.; Leahy, DC.; Coorey, NV.; Bellows, DS.; Atkinson, PH. and Miller, JH. (2013). Laulimalinde and peloruside A inhibit mitosis of Saccharomyces cerevisiae by preventing microtubule depolymerisation-dependent steps in chromosome separation and nuclear positioning. Molecular Biosystems. 9(11): 2842-2852

Bianchi, MM.; Sartori, G.; Vandenbol, M.; Kaniak, A.; Uccelletti, D.; Mazzaoni, C.; di Rago, JP.; Garignani, G.; Slonimski, PP. and Frontali, L. (1999). How to bring orphan genes into functional families. Yeast. 15(6): 513-526

Bilsland, E.; Hult, M.; Bell, SD.; Sunnerhagen, P. and Downs, JA. (2007). The Bre5/Ubp3 ubiquitin protease complex from budding yeast contributes to the cellular response to DNA damage. DNA Repair. 6(10): 1471-1484

Bircham, PW.; Maass, DR.; Roberts, CA.; Kiew, PY.; Low, YS.; Yegambaram, M.; Matthews, JH.; Jack, CA. and Atkinson, PH. (2011). Secretory pathway genes assessed by high-throughput microscopy and synthetic genetic array analysis. Molecular Biosystems. 7(9): 2589-2598

Blunt, JW.; Copp, BR.; Keyzers, RA.; Munro, MHG. and Prinsep, MR. (2012). Marine natural products. Natural Products Reports. 29: 144-222

Boman, HG. (1991). Antibacterial peptides: key components needed in immunity. Cell. 65: 205-207

Boone, C.; Bussey, H. and Andrews, BJ. (2007). Exploring genetic interactions and networks with yeast. Nature Reviews: Genetics. 8: 437-449

Bordallo, J.; Bordallo, C.; Gascon, S.; Suarez-Rendueles, P. (1991). Molecular cloning and sequencing of genomic DNA encoding yeast vacuolar carboxypeptidase yscS. FEBS Letters. 283(1): 2732

Brackovic, A. and Harvey, JE. (2015). Synthetic, semisynthetic and natural analogues of peloruside A. Chemical Communications (Camb). 51(23): 4750-4765

Breter, HJ.; Ferguson, J.; Peterson, TA. and Reed, SI. (1983). Isolation and transcriptional characterization of three genes with function at start, the controlling event of the 
Saccharomyces cerevisiae cell division cycle: CDC36, CDC37 and CDC39. Molecular and Cellular Biology. 3(5): 881-891

Brune, K. and Hinz, B. (2004). The discovery and development of anti-inflammatory drugs. Arthritis \& Rheumatism. 50(8): 2391-2399

Cairns, BR.; Kim, YJ.; Sayre, MH.; Lauren, BC. and Kornberg, RD. (1994). A multisubunit complex containing the SWI1/ADR6, SWI2/SNF2, SWI3, SNF5 and SNF6 gene products isolated from yeast. Proceedings of the National Academy of Science. 91(5): 1950-1954

Callegari, S.; McKinnon, RA.; Andrews, S.; de Barros Lopes, MA. (2010). Atorvastatin-induced cell toxicity in yeast is linked to disruption of protein isoprenylation. FEMS Yeast Research. 10(2): 188-198

Calzada, F.; Cerda-Garcia-Rojas, CM.; Meckes, M.; Cedillo-Rivera, R.; Bye, R. and Mat, R. (1999). Geranins A and B, new antiprotozoal A-type proanthocyanidins from Geranium niveum. Journal of Natural Products. 62: 705-709

Calzada, F.; Cedillo-Rivera, R. and Mata, R. (2001). Antiprotozoal activity of the constituents of Conyza filaginoids. Journal of Natural Products. 64: 671-673

Campbell, WC. (2012). History of avermectin and ivermectin, with notes on the history of other macrocyclic lactone antiparasitic agents. Current Pharmaceutical Biotechnology. 13(6): 853865

Carbrera, M.; Arlt, H.; Epp, N.; Lachmann, J.; Griffith, J.; Perz, A.; Reggiori, F. and Ungermann, C. (2013). Functional separation of endosomal fusion factors and the class $\mathrm{C}$ core vacuole/endosome tethering (CORVET) complex in endosome biogenesis. Journal of Biological Chemistry. 288(7): 5166-5175

Carmen, AA.; Rundlett, SE. and Grunstein, M. (1996). HDA1 and HDA3 are componenets of a yeast histone deacetylase (HDA) complex. Journal of Biological Chemistry. 271(26): 15837-15844

Carroll, SY.; Stirling, PC.; Stimpson, HE.; Giesselmann, E.; Schmitt, MJ. and Drubin, DG. (2009). A yeast killer toxin screen provides insights into a/b toxin entry, trafficking, and killing mechanisms. Developmental Cell. 17(4): 552-560

Chabes, A.; Domkin, V. and Thelander, L. (1999). Yeast Sml1, a protein inhibitor of ribonucleotide reductase. Journal of Biological Chemistry. 274(51): 36679-36683

Chen, OS.; Crisp, RJ.; Valachovic, M.; Bard, M.; Winge, DR. and Kaplan, J. (2004). Transcription of the yeast iron regulon does not respond directly to iron but rather to iron-sulfur cluster biosynthesis. Journal of Biological Chemistry. 279(28): 29513-29518 
Collart, MA. and Struhl, K. (1994). NOT1 (CDC39), NOT2 (CDC36), NOT3 and NOT4 encode a globalnegative regulator of transcription that differentially affects TATA-element utilization. Genes \& Development. 8(5): 525-537

Connor, JR.; Menzies, SL.; St. Martin, SM. and Mufson, EJ. (1992). A histochemical study of iron, transferring, and ferritin in Alzheimer's diseased brains. Journal of Neuroscience Research. 31(1): 75-83

Coorey, NV.; Matthews, JH.; Bellows, DS. and Atkinson, PH. (2015). Pleiotropic drug-resistance attenuated genomic library improves elucidation of drug mechanisms. Molecular Biosystems. 11(11): 3129-3136

Cos, P.; de Bruyne, N.; Hermans, S.; Apers, D.; Berghe, V. and Vlietinck, AJ. (2003). Proanthocyanidins, in helath care current and new trends. Current Medicinal Chemistry. 11(10): $1345-1359$

Cox, PA.; Sperrym LR.; Tuominen, M. and Bohlin, L. (1989). Pharmacological activity of the Samoan ethnopharmacopoeia. Economic Botany. 43(4): 487-497

Cragg, GM. and Newman, DJ. (2013). Natural products: a continuing source of novel drug leads. Biochimica Biophysica Acta 1830(6): 3670-3695

Cragg, GM.; Newman, DJ. and Snader, KM. (1997). Natural products in drug discovery and development. Journal of Natural Products. 60(1): 52-60

Craven, RJ.; Mallory, JC. and Hand, RA. (2007). Regulation of iron homeostasis mediated by the heme-binding protein Dap1 (damage resistance protein 1) via the P450 protein Erg11/Cyp51. Journal of Biological Chemistry. 282(50): 36543-36551

Cui, L. and Su, XZ. (2009). Discovery, mechanisms or action and combination therapy of artemisinin. Expert Review of Anti-infective Therapy. 7(8): 999-1013

Cullen, LM.; Gao, X.; Easteal, S. and Jazwinska, EC. (1998). The hemochromatosis 845 G>A and 187 $\mathrm{C}>\mathrm{G}$ mutations: prevalence in non-Caucasian populations. American Journal of Human Genetics. 62(6): 1403-1407

Cushman, DW. and Ondetti, MA. (1999). Design of angiotensin converting enzyme inhibitors. Nature Medicine. 5(10): 1110-1113

Cyr, DM.; Langer, T. and Douglas, MG. (1994). DnaJ-like proteins: molecular chaperones and specific regulators of Hsp70. Trends in Biochemical Sciences. 19(4): 176-181 
Dai, J. and Mumper, R. (2010). Phant phenolics: Extraction, analysis and their antioxidant and anticancer properties. Molecules. 15(10): 7313-7352

Davies, MK. and Hollman, A. (2002). The opium poppy, morphine, and verapamil. Heart. 88: 3

De Bruyne, T.; Pieters, L.; Witvrouw, M.; de Clercq, E.; van den Berghe, D. and Vlietinck, AJ. (1999). Biological evaluation of proanthocyanidin dimers and related polyphenols. Journal of Natural Products. 62(7): 954-958

De Silva, DM.; Askwith, CC.; Eide, D. and Kaplan, J. (1995). The FET3 gene product required for high affinity iron transport in yeast is a cell surface ferroxidase. Journal of Biological Chemistry. 270(3): 1098-1101

Dexter, DT.; Carayon, A.; Javoy-Agid, F.; Agid, Y.; Wells, FR.; Daniel, SE.; Lees, AJ.; Jenner, P. and Marsden, CD. (1991). Alternations in the levels of iron, ferritin and other trace metals in Parkinson's disease and other neurodegenerative diseases affecting the basal ganglia. Brain. 114(4): 1953-1975

Dhandapani, R. and Sabna, B. (2008). Phytochemical constituents of some Indian medicinal plants. Ancient Science of Life. 27(4): 1-8

Dias, DA.; Urban, S. and Roessner, U. (2012). A historical overview of natural products in drug discovery. Metabolites. 2(2): 303-336

Dittmar, JC.; Reid, RJD. and Rothstein, R. (2010). ScreenMill: a freely available software suite for growth measurement, analysis and visualization of high-throughput screen data. $B M C$ Bioinformatics. 11: 353

Dix, DR.; Bridgham, JT.; Broderius, MA.; Byersdorfer, CA. and Eide, DJ. (1994). The FET4 gene encodes the low affinity Fe(II) transport protein of Saccharomyces cerevisiae. Journal of Biological Chemistry. 269(42): 26092-26099

Dix, DR.; Bridgham, J.; Broderius, M. and Eide, DJ. (1997). Characterization of the FET4 protein of yeast. Evidence for a direct role in the transport of iron. Journal of Biological Chemistry. 272(18): 11770-11777

Dudley, AM.; Janse, DM.; Tanay, A.; Shamir, R. and Church, GM. (2005). A global view of pleiotropy and phenotypically derived gene function in yeast. Molecular Systems Biology. 1: 1-11

Duffy, SJ.; Biegelsen, ES.; Holbrook, M.; Russell, JD.; Gokce, N.; Keaney, JF. and Vita, JA. (2001). Iron chelation improves endothelial function in patients with coronary artery disease. Ciculation. 103: $2799-2804$ 
Dunn, B. and Wobbe, CR. (2001). Preparation of protein extracts from yeast. Current Protocols in Molecular Biology. Chapter 13: Unit 13

Dunstan, CA.; Noreen, Y.; Serrano, G.; Cox, PA.; Perera, P. and Bohlin, L. (1997). Evaluation of some Samoan and Peruvian medicinal plants by prostaglandin biosynthesis and rat ear oedema assays. Journal of Ethnopharmacology. 57(1): 35-56

Eide, DJ. (1998). The molecular biology of metal iron transport in Saccharomyces cerevisiae. Annual Reviews. 18: 441-469

Endo, A. (2010). A historical perspective on the discovery of statins. Proceedings of the Japan Academy, Series B Physical and Biological Sciences. 86(5): 484-493

Eng, J.; Kleinman, W.; Singh, L.; Sings, G. and Raufman, J. (1992). Isolations and characterization of exendin-4, an exendin-3 analog from Heloderma suspectum venom. Journal of Biological Chemistry. 267(11): 7402-7405

Esposito, E. and Cuzzocrea, S. (2009). TNF-alpha as a therapeutic target in inflammatory diseases, ischemia-reperfusion injury and trauma. Current Medicinal Chemistry. 16(24): 3152-3167

Esposito, AM. and Kinzy, TG. (2010). The eukaryotic translation elongation factor 1Bgamma has a non-guanine nucleotide exchange factor role in protein metabolism. Journal of Biological Chemistry. 285(49): 37995-38004

Faa, G. and Crisponi, G. (1999). Iron chelating agents in clinical practice. Coordination Chemistry Reviews. 184: 291-310

Falagas, ME.; McDermott, L. and Snydman, DR. (1997). Effect of $\mathrm{pH}$ on in vitro antimicrobial susceptibility of the Bacteroids fragilis group. Antimicrobial Agents and Chemotherapy. 41(9): 2047-2049

Faulkner, DJ. (2000). Highlights of marine natural products chemistry (1972-1999). Natural Products Reports. 17(1): 1-6

Feng, J.; Yang, XW. and Wang, RF. (2011). Bio-assay guided isolation and identification of $\alpha$ glucosidase inhibitors from the leaves of Aquilaria sinensis. Phytochemistry. 72(2): 242-247

Ferreira, SH.; Bartlet, DC. and Greene, LJ. (1970). Isolation of bradykinin-potentiating peptides from Bothrops jararaca venom. Biochemistry. 9(13): 2583-2593

Fisk, DG., Ball, CA.; Dolinski, K.; Engel, SR.; Hong, EL.; Issel-Tarver, L.; Schwartz, K.; Sethuraman, A.; Botstein, D.; Cherry, JM.; Saccharomyces Genome Database Project. (2006). Saccharomyces cerevisiae S288C genome annotation: a working hypothesis. Yeast. 23(12): 857-865 
Foury, F. (1997). Human genetic diseases: a cross-talk between man and yeast. Gene. 195(1): 1-10

Fry, RC.; Begley, TJ. and Samson, LD. (2005). Genome-wide responses to DNA-damaging agents. Annual Reviews of Microbiology. 59: 357-377

Fu, D.; Beller, TJ. and Dunn, TM. (1995). Sequence, mapping and disruption of CCC2, a gene that cross-complements the $\mathrm{Ca}(2+)$-sensitive phenotype of csg1 mutants and encodes a P-type ATPase belonging to the $\mathrm{Cu}(2+)$-ATPase subfamily. Yeast. 11(3): 283-292

Fu, D. and Richardson, DR. (2007). Iron chelation and regulation of the cell cycle: 2 mechanisms of posttranscriptional regulation of the universal cyclin-dependent kinase inhibitor p21CIP1/WAF1 by iron depletion. Blood. 110(2): 752-761

Ganz, T. and Nemeth, E. (2015). Iron homeostasis in host defense and inflammation. Nature Reviews: Immunology. 15(8): 500-510

Gardener, RG.; Swarbrick, GM.; Bays, NW.; Cronin, SR.; Wilhovsky, S.; Seelig, L.; Kim, C. and Hampton, RY. (2000). Endoplasmic reticulum degradation requires lumen to cytosol signaling. Transmembrane control of Hrd1p by Hrd3p. Journal of Cell Biology. 151(1): 6982

Ghaemmaghami, S. Huh, WK.; Bower, K.; Howson, RW.; Belle, A.; Dephoure, N.; O'Shea, EK. and Weissman, JS. (2003). Global analysis of protein expression in yeast. Nature. 425(6959): 737-741

Giaever, G.; Shoemaker, DD.; Jones, TW.; Liang, H.; Winzeler, EA.; Astromoff, A. and Davis, RW. (1999). Genomic profiling of drug sensitivities via induced haploinsufficiency. Nature Genetics. 21(3): 278-283

Giaever, G.; Chu, AM.; Ni, L.; Connelly, C.; Riles, L.; Veronneau, S.; Dow, S.; Lucau-Danila, A.; Anderson, K.; Andrew, B.; Arkin, AP.; Stromoff, A.; El-Bakkoury, M.; Bangham, R.; Benito, R.; Brachat, S.; Campanaro, S.; Curtiss, M.; Davis, K.; Deutschbauer, A.; Entian, KD.; Flaherty, P.; Foury, F.; Garfinkel, DJ.; Gerstein, M.; Gotte, D.; Guldener, U.; Hegemann, JH.; Hempel, S.; Herman, Z.; Jaramillo, DF.; Kelly, SL.; Kotter, P.; LaBonte, D.; Lamb, DC.; Lan, N.; Liang, J.; Liao, J.; Liu, L.; Luo, C.; Lussier, M; Mao, R.; Menard, P.; Ppo, SL.; Revuelta, JL.; Roberts, CJ.; Rose, M.; Ross-MacDonald, P.; Scherens, B.; Schimmack, G.; Shafer, B.; Shoemaker, DD.; Sookhai-Mahadeo, S.; Storms, RK.; Strathern, JN.; valle, G.; Voet, M.; Volckaert, G.; Wang, CY.; Ward, TR.; Wilhelmy, J.; Winzeler, EA.; Yang, Y.; Yen, G.; Youngman, E.; Yu, K.; Bussey, H.; Boeke, JD.; Snyder, M.; Phillippsen, P.; Davis, RW. and Johnston, M. (2002). Functional profiling of the Saccharomyces cerevisiae genome. Nature. 418(6896): 387-391 
Giaver, G.; Flaherty, P.; Kumm, J.; Proctor, M.; Nislow, C.; Jaramillo, DF.; Chu, AM.; Jordan, MI.; Arkin, AP. and Davis, RW. (2004). Chemogenomic profiling: identifying the functional interactions of small molecules in yeast. Proceedings of the National Academy of Science. 101(3): 793798

Goebl, MG.; Yochem, J.; Jentsch, S.; McGrath, JP.; Varshavsky, A. and Byers, B. (1988). The yeast cell cycle gene CDC34 encodes a ubiquitin-conjugating enzyme. Science. 241(4871): 1331-1335

Goffeau, A.; Barrell, BG.; Bussey, J.; Davis, RW.; Dujon, B.; Feldman, H.; Galiber, F.; Hoheisel, JD.; Jacq, C.; Johnston, M.; Louis, EJ.; Mewes, JW.; Murakami, Y.; Philippsen, P.; Tettelin, J. and Oliver, SG. (1996). Life with 6000 genes. Science. 274(5287): 563-567

Gollub, EG.; Liu, KP.; Dayan, J.; Adlersberg, M. and Sprinson, DB. (1977). Yeast mutants deficient in heme biosynthesis and a heme mutant additionally blocked in cyclization of 2,3oxidosqualene. Journal of Biological Chemistry. 252(9): 2840-2854

Graack, HR. and Wittmann-Liebold, B. (1998). Mitochondrial ribosomal proteins (MRPs) of yeast. Biochemical Journal. 329 (Pt 3): 433-448

Graziano, BR.; Jonasson, EM.; Pullen, JG.; Gould, CJ. and Goode, BL. (2013). Ligand-induced activation of a formin-NPF pair leads to collaborative actin nucleation. Journal of Cell Biology. 201(4): 595-611

Gross, C.; Kelleher, M.; Iyer, VR.; Brown, PO. and Winge, DR. (2000). Identification of the copper regulon in Saccharomyces cerevisiae. Journal of Biological Chemistry. 275(41): 32310-32316

Guo, W.; Grant, A. and Novick, P. (1999). Exo84p is an exocyst protein essential for secretion. Journal of Biological Chemistry. 274(33): 23558-23564

Gustafon, KR.; Cardellin, JH 2nd, McMahon, JB.; Gluakowski, RJ.; Ishitoya, J.; Szallasi, Z.; Lewin, NE.; Blumberg, PM.; Weislow, OS.; Beutler, JA. (1992). A nonpromoting phorbol from the Samoan medicinal plant Homalanthus nutans inhibits cell killing by HIV-1. Journal of Medicinal Chemistry. 35(11): 1978-1986

Hagerman, AE.; Riedl, KM.; Jones, GA.; Sovik, KN.; Rithcard, NT.; Hartzfeld, PW. and Riechel, TL. (1998). High molecular weight plant polyphenolics (tannins) as biological antioxidants. Journal of Agricultural and Food Chemistry 46(5): 1887-1892

Hammer, KA.; Carson, CF. and Riley, TV. (1999). Antimicrobial activity of essential oils and other plant extracts. Journal of Applied Microbiology. 86(6): 985-990 
Hand, RA.; Jia, N.; Bard, M. and Craven, RJ. (2003). Saccharomyces cerevisiae Dap1p, a novel DNA damage response protein related to the mammalian membrane-associated progesterone receptor. Eukaryotic Cell. 2(2): 306-317

Hartung, EF. (1954). History of the use of colchicum and related medicaments in gout. Annals of Rheumatic Diseases. 13(3): 190-200

Harrington, MT. (2001). Samoan medicinal plants and their usage. $2^{\text {nd }}$ ed. Agricultural Development in the American Pacific Project, Honolulu (HI). 81 pages

Harvey, AL. (2008). Natural products in drug discovery. Drug Discovery Today. 13(19-20): 894-901

Hassett, R.; Dix, DR.; Eide, DJ. and Kosman, DJ. (2000). The Fe(II) permease Fet4p functions as a low affinity copper transporter and supports normal copper trafficking in Saccharomyces cerevisiae. Biochemical Journal. 351 (Pt 2): 477-484

Hatcher, HC.; Planalp, R.; Cho, J.; Torti, FM. and Torti, SV. (2008). Curcumin: from ancient medicine to current clinical trials. Cell and Molecular Life Sciences 65(11): 1631-1652

Hatcher, HC.; Singh, RN.; Torti, FM. And Torti, SV. (2009). Synthetic and natural iron chelators: therapeutic potential and clinical use. Future Medicinal Chemistry 1(9): 1643-1670

Heim, KE.; Tagliaferro, AR. and Bobilya, DJ. (2002). Flavonoid antioxidants: chemistry, metabolism and structure-activity relationships. Journal of Nutritional Biochemistry 13(10): 572-584

Heinrich, M. and Teoh, LH. (2004). Galanthamin from snowdrop-the development of a modern drug against Alzheimer's disease from local Caucasian knowledge. Journal of Ethnopharmacology. 92(2-3): 147-162

Heymann, P.; Ernst, JF. and Winkelmann, G. (2000). Identification and substrate specificity of a ferrichrome-type siderophore transporter (Arn1p) in Saccharomyces cerevisiae. FEMS Microbiology Letter. 186(2): 221-227

Hezareh, M. (2005). Prostratin as a new therapeutic agent targeting HIV viral reservoirs. Drug News \& Perspectives. 18(8): 496-500

Hider, RC.; Liu, ZD. and Khodr, HH. (2001). Metal chelation of polyphenols. Methods in Enzymology 335: 109-203

Hillenmeyer, ME.; Fung, E.; Wildenhain, J.; Pierce, SE.; Hoon, S.; Lee, W.; Proctor, M.; St Onge, RP.; Tyers, M.; Koller, D.; Altman, RB.; Davis, RW.; Nislow, C. and Giaever, G. (2008). The chemical genomic portrait of yeast: uncovering a phenotype for all genes. Science. 320(5874): 362365 
Hillenmeyer, ME.; Ericson, E.; Davis, RW.; Nislow, C.; Koller, D. and Giaever, G. (2010). Systematic analysis of genome-wide fitness adata in yeast reveals novel gene function and drug action. Genome Biology. 11(3): R30

Hoyt, MA.; Totis, L. and Roberts, BT. (1991). S. cerevisiae genes required for cell cycle arrest in response to loss of microtubule function. Cell. 66(3): 507-517

Huang, GZ.; Luo, SM. and Zeng, XW. (2012). Effects of plant extracts from grapeseed on growth and composition of muscles of hybrid Crucian carp. Chinese Journal of Fisheries and Science. 31: 433-436

Huh, WK.; Falvo, JV.; Gerke, LC.; Carroll, AS.; Howson, RW.; Weissman, JS. and O'Shea, EK. (2003). Global analysis of protein localization in budding yeast. Nature. 425(6959): 686-691

Inadome, H.; Noda, Y.; Adachi, J. and Yoda, K. (2005). Immunoisolation of the yeast Golgi subcomparments and characterization of a novel membrane protein, Svp26, discovered in the Sed5-containing compartments. Molecular and Cellular Biology. 25(17): 7696-7710

Inoue, H. and Tanabe, T. (1998). Transcriptional role of the nuclear factor kappa B site in the induction by lipopolysaccharide and suppression by dexamethasone of cyclooxygenase-2 in U937 cells. Biochemical and biophysical Research Communications. 244(1): 143-148

Ishimi, Y. and Kikuchi, A. (1991). Identification and molecular cloning of yeast homolog of nucleosome assembly protein I which facilitates nucleosome assembly in vitro. Journal of Biological Chemistry. 266(11): 7025-7029

Islam, S.; Jarosch, S.; Zhou, J.; Parquet-Mdel, C.; Toquri, JT.; Colp, P. Holbein, BE. and Lehmann, C. (2016). Anti-inflammatory and anti-bacterial effects of iron chelation in experimental sepsis. Journal of Surgical Research. 200(1): 266-273

Itokawa, H.; Wang, X. and Lee, K-H. (2005). Homoharringtonine and related compounds. In: Cragg, GM.; Kingston, DGI. and Newman, DJ. editors. Anticancer agents from natural products. CRC/Taylor \& Fracis; Boca Raton, Florida. Pp47-70

Ivessa, SA.; Zhou, JQ. and Zakian, VA. (2000). The Saccharomyces Pif1p DNA helicase and the highly related Rrm3p have opposite effects on replication fork progression in ribosomal DNA. Cell. 100(4): 479-489

Johnson, HE.; Banack, SA. and Cox, PA. (2008). Variability in content of the anti-AIDS drug candidate prostratin in Samoan populations of Homalanthus nutans. Journal of Natural Products. 71(12): 2041-2044 
Jong, AY. and Campbell, JL. (1986). Isolation of the gene encoding yeast single-stranded nucleic acid binding protein 1. Proceedings of the National Academy of Science. 83(4): 877-881

Jurado, RL. (1997). Iron, infections, and anemia of inflammation. Clinical Infectious Diseases: An official publication of the Infectious Diseases Society of America. 25(4): 888-895

Kabera, J.; Tuisenge, R.; Ugirinshuti, V.; Nyirabageni, A. and Munyabuhoro, S. (2014). Preliminary investigation on antihelmintic activity and phytochemical screening of leaf crude extracts of Tithonia diversifolia and Tephrosia vogelii. African Journal of Microbiological Research. 8(25): 2449-2457

Kaplan, J.; McVey-Ward, D.; Crisp, RJ. and Philpott, CC. (2006). Iron-dependent metabolic remodeling in S. cerevisiae. Biochimica Biophysica Acta. 1763(7): 646-651

Kaufman, TS. and Ruveda, EA. (2005). The quest for quinine: those who won the battles and those who won the war. Angewandte Chemie (International ed. in English). 44(6): 854-885

Kell, DB. (2009). Iron behaving badly: inappropriate iron chelation as a major contributor to the aetiology of vascular and other progressive inflammatory and degenerative diseases. $B M C$ Medical Genomics 2: 2

Khandababee, K. and van Ree, T. (2001). Tannins: classification and definition. Natural Products Reports. 18(6): 641-649

Kinzy, TG.; Ripmaster, TL. And Woolford, JL Jr. (1994). Multiple genes encode the translation elongation factor EF-1 gamma in Saccharomyces cerevisiae. Nucleic Acids Research. 22(13): 2703-2707

Koehn, FE. and Carter, GT. (2005). The evolving role of natural products in drug discovery. Nature Reviews: Drug Discovery. 4(3): 206-220

Kolodziej, H.; Kayser, O.; Latte, KP. And Ferreira, D. (1999). Evaluation of the antimicrobial potency of tannins and related compounds using the microdilution broth method. Planta Medicine. 65: $444-446$

Kolodziej, H.; Kayser, O.; Kiderlen, AF.; Ito, H.; Hatano, T. and Yoshida, T. (2001). Proanthocyanidins and related compounds: Antileishmanial activity and modulatory effects on nitric oxide and tumour necrosis factor-alpha-release in the murine macrophage-like cell line RAW 264.7. Biological \& Pharmaceutical Bulletin. 24(9): 1016-1021

Kontiokari, T.; Svanberg, M.; Mattila, P.; Leinonen, M. and Uhari, M. (1999). Quantitative analysis of the effect of xylitol on pneumococcal nasal colonization in rats. FEMS Microbiology Letters. 178(2): 313-317 
Kraft, C.; Deplazes, A.; Sohrmann, M. and Peter, M. (2008). Mature ribosomes are selectively degraded upon starvation by an autophagy pathway requiring the Ubp3p/Bre5p ubiquitin protease. Nature: Cell Biology. 10(5): 602-610

Krogan, NJ.; Kim, M.; Ahn, SH.; Zhong, G.; Kobor, MS.; Cagney, G.; Emili, A.; Shilatifard, A.; Buratowski, S. and Greenblatt, JF. (2002). RNA polymerase II elonogation factors of Saccharomyces cerevisiae: a targeted proteomics approach. Molecular and Cellular Biology. 22(20): 69796992

Kumar, VP.; Neelam, SC.; Harish, P. and Rajani, M. (2006). Search for antibacterial and antifungal agents from selected Indian medicinal plants. Journal of Ethnopharmacology. 107: 182-188

Lahlou, M. (2013). The success of natural products in drug discovery. Pharmacology \& Pharmacy. 4: $17-31$

Lavoie, S.; Ouellet, M.; Fleury, PY.; Gauthier, C.; Legault, J. and Pichette, A. (2015). Complete 1H and 13C NMR assignments of a series of pergalloylated tannins. Magnetic Resonance Chemistry. 54: $168-174$

Lee, CC.; Hsieh, HJ.; Hsieh, CH. and Hwang, DF. (2014). Antioxidative and anticancer activities of various ethanolic extract fractions from crown-of-thorn starfish (Acanthaster planci). Environmental Toxicology \& Pharmacology. 38(3): 761-773

Lee, CC.; Hsieh, HJ.; Hsieh, CH. and Hwang, DF. (2014). Spine venom of crown-of-thorns starfish (Acanthaster planci) induces antiproliferation and apoptosis of human melanoma cells (A375.S2). Toxicon: Official Journal of the International Society on Toxinology. 91: 126-134

Lee, CC.; Hsieh, HJ. and Hwang, DF. (2015). Cytotoxic and apoptotic activities of the plancitoxin I from the venom of crown-of-thorns starfish (Acanthaster planci). On A375.S2 cells. Journal of Applied Toxicology. 35(4): 407-417

Lehmann, C.; Islam, S.; Jarosch, S.; Zhou, J.; Hoskin, D.; Greenshields, A.; Al-Banna, N.; Sharawy, N.; Sczcesniak, A.; Kelly, M; Wafa, K.; Cheliak, W. and Holbein, B. (2015). The utility of iron chelators in the management of inflammatory disorders. Mediators of Inflammation. Vol 2015, Article 516740, 12 pages

Lewis, TA.; Taylor, FR. and Parks, LW. (1985). Involvement of heme biosynthesis in control of sterol uptake by Saccharomyces cerevisiae. Journal of Bacteriology. 163(1): 199-207

Li, WG.; Zhang, XY.; Wu, YJ. and Tian, X. (2001). Anti-inflammatory effects and mechanism of proanthocyanidins from grape seeds. Acta Pharmacology Sin. 22(12): 1117-1120 
Li, W.; Mo, W.; Shen, D.; Sun, L.; Wang, J.; Lu, S.; Gitschier, JM. and Zhou, B. (2005). Yeast model uncovers dual roles of mitochondrial in the action of artemisinin. PLoS Genetics. 1(3): e36

Li, J. (2010). Grape seed proanthocyanidins ameliorate doxorubicin-induced cardiotoxicity. American Journal of Chinese Medicine. 38(3): 569

Lill, R. and Mühlenhoff, U. (2008). Maturation of iron-sulfur proteins in eukaryotes: mechanisms, connected processes and diseases. Annual Reviews 77: 669-700

Lin, SJ.; Pufahl, DA.; Dancis, A.; O’Halloran, TV. and Culotta, VC. (1997). A role for the Saccharomyces cerevisiae ATX1 gene in copper trafficking and iron transport. Journal of Biological Chemistry. 272(12): 9215:9220

Lo, WS. and Dranginis, AM. (1996). FL011, a yeast gene related to the STA genes, encodes a novel cell surface flocculin. Journal of Bacteriology. 178(24): 7144-7151

Lopez, A.; Parsons, AB.; Nislow, C.; Giaever, G. and Boone, C. (2008). Chemical-genetic approaches for exploring the mode of action of natural products. Progress in Drug Research. 66(237): 239-271

Lum, PY.; Armour, CD.; Stepaniants, SB.; Cavet, G.; Wolf, MK.; Butler, JS.; Hinshaw, JC.; Garnier, P.; Preswich, GD.; Leonardson, A.; Garrett-Engele, P.; Rush, CM.; Bard, M.; Schimmack, G.; Phillips, JW.; Roberts, CJ. and Shoemaker, DD. (2004). Discovering the modes of action for the therapeutic compounds using a genome-wide screen of yeast heterozygotes. Cell. 116(1): 121-137

Ma, P.; Winderickx, J.; Nauwelaers, D.; Dumotier, F.; De Doncker, A.; Thevelein, JM. and Van Dijck, P. (1999). Deletion of SFI1, a novel suppressor of partial Ras-cAMP pathway deficiency in the yeast Saccharomyces cerevisiae, causes G(2) arrest. Yeast. 15(11): 1097-1109

Mack, D.; Nishimura, K.; Dennehey, BK.; Arbogast, T.; Parkinson, J.; Toh-e, A.; Pringle, JR.; Bender, A. and Matsui, Y. (1996). Identification of the bud emergence gene BEM4 and its interactions with rho-type GTPases in Saccharomyces cerevisiae. Molecular \& Cellular Biology. 16(8): 4387-4895

Macpherson, M. and Macpherson, L. (1990). Samoan medical belief and practice. Auckland University Press, New Zealand. 272 pages.

Mahmoudian, M. and Rahimi-Moghaddam, P. (2009). The anti-cancer activity of noscapine: a review. Recent Patents on Anti-Cancer Drug Discovery. 4(1): 92-97 
Mallory, JC.; Crudden, G.; Johnson, BL.; Mo, C.; Pierson, CA.; Bard, M and Craven, RJ. (2005). Dap1p, a heme-binding protein that regulates the cytochrome P450 protein Erg11p/Cyp51p in Saccharomyces cerevisiae. Molecular \& Cellular Biology. 25(5): 1669-1679

Malone, EA.; Clark, CD.; Chiang, A. and Winston, F. (1991). Mutations in SPT16/CDC68 suppress cisand trans-acting mutations that affect promoter function in Saccharomyces cerevisiae. Molecular \& Cellular Biology. 11(11): 5710-5717

Mbah, JA.; Ngemenya, MN.; Abawah, AL.; Babiaka, SB.; Nubed, LN.; Nyongbela, KD.; Lemuh, ND. And Efange, SMN. (2012). Bioassay-guided discovery of antibacterial agents: in vitro screening of Peperomia vulcanica, Peperomia fernandopoioana and Scleria striatinux. Annals of Clinical Microbiology and Antimicrobials. 11: 10

McGivern, JG. (2007). Ziconotide: a review of its pharmacology and use in the treatment of pain. Neuropsychiatric Disease \& Treatment. 3(1): 69-85

Menon, VP. and Sudheer, AR. (2007). Antioxidant and anti-inflammatory properties of curcumin. Advances in Experimental Medicine and Biology. 595: 105-125

Meshnick, SR. and Dobson, MJ. (2001). The history of antimalarial drugs. In Antimalarial Chemotherapy: Mechanisms of Action, Resistance and New Directions in Drug Discovery. Ed Rosenthal, PJ. Totowa NJ: Humana Press Inc. pp 15-25

Miller, EL. (2002). The penicillins: a review and update. Journal of Midwifery \& Women's Health. 47(6): 426-434

Minear, S.; O’Donnell, AF.; Ballew, A.; Giaever, G.; Nislow, C.; Stearns, T.; Cyert, MS. (2011). Curcumin inhibits growth of Saccharomyces cerevisiae through iron chelation. Eukaryotic Cell. 10(11): 1574-1581

Mishra, LC.; Sing, BB. and Dagenais, S. (2001). Ayerveda-A historic perspective and principles of traditional health care systems in India. Alternative Therapies in Health and Medicine. 7(2): 36-42

Miszkiel, KA.; Paleny, MN.; Wilkinson, ID.; Hall-Crags, MA.; Ordidge, R.; Kendall, BE.; Miller, RF. and Harrison, MJ. (1997). The measurement of R2, RE* and RE' in HIV-infected patients using the prime sequence as a measure of brain iron depositition. Magnetic Resonance Imaging. 15(10): $1113-1119$

Moini, H.; Guo, Q. and Packer, L. (2002). Xanthine oxidase and xanthine dehydrogenase inhibition by the procyanidin-rich French maritime pine bark extract, Pycnogenol ®: A protein binding effect. In Flavonoids in Cell Function. Eds Buslig, B. and Manthey, J. New York: Kluwer Academic/Plenum Publishers. pp141-149 
Molan, PC. (2001). Potential of honey in the treatment of wounds and burns. American Journal of Clinical Dermatology. 2(1): 13-19

Molina-Holgado, F.; Gaeta, A.; Francis, PT.; Williams, RJ. and Hider, RC. (2008). Neuroprotective actions of deferiprone in cultured cortical neuroles and SHSY-5Y cells. Journal of Neurochemistry. 105(6): 2466-2476

Molinski, TF.; Dalisay, DS.; Lievens, SL. and Saludes, JP. (2009). Drug development from marine natural products. Nature Reviews: Drug Discovery. 8: 69-85

Monahan, BJ.; Villen, J.; Marguerat, S.; Bahler, J.; Gygi, SP. and Winston, F. (2008). Fission yeast SWI/SNF and RSC complexes show compositional and functional differences from budding yeast. Nature: Structural \& Molecular Biology. 15(8): 873-880

Morel, I.; Lescoat, G.; Cogrel, P.; Sergent, O.; Pasdeloup, N.; Brissot, P.; Cillard, P. and Cillard. J. (1993). Antioxidant and iron chelating activities of the flavonoids catechin, quercetin and diosmetin on iron-loaded rat hepatocyte cultures. Biochemical Pharmacology 45(1): 13-19

Muren, E.; Oyen, M.; Barmark, G. and Ronne, H. (2001). Identification of yeast deletion strains that are hypersensitive to brefeldin A or monensin, two drugs that affect intracellular transport. Yeast. 18(2): 163-172

Nanjo, F.; Goto, K.; Seto, R.; Suzuki, M.; Sakai, M. and Hara, Y. (1996). Scavenging effects of tea catechins and their derivatives on 1,1-diphenyl-2-picrylhydrazyl radical. Free Radical Biology \& Medicine. 21(6): 895-902

Nelson, ML. and Levy, ST. (2011). The history of tetracyclines. Annals of the New York Academy of Sciences. 124: 17-32

Newton, R.; Kuitert, LM.; Bergmann, M.; Adcock, IM. and Barnes, PJ. (1997). Evidence for involvement of NF-kappaB in the transcriptional control of COX-2 gene expression by IL-1 beta. Biochemical \& Biophysical Research Communications. 237(1): 28-32

Niedenthal, RK.; Riles, L.; Johnston, M. and Hegemann, JH. (1996). Green fluorescent protein as a marker for gene expression and subcellular localization in budding yeast. Yeast. 12(8): 773786

Nislow, C.; Ray, E. and Pillus, L. (1997). SET1, a yeast member of the trithorax family, functions in transcriptional silencing and diverse cellular processes. Molecular \& Cellular Biology. 8(12): $2421-2436$

Njoya, EM.; Weber, C.; Hernandez-Cuevas, NA.; Hon, CC.; Janin, Y.; Kamini, MFG.; Moundipa, PF.; Guillen, N. (2014). Bioassay-guided fractionation of extracts from Codeaeum variegatum 
against Entamoeba histolytica discovers compounds that modify expression of ceramide biosynthesis related genes. PLoS Neglected Tropical Diseases. 8(1): e2607

Nobel Prize Assembly. (2015). The Nobel Prize in Physiology or Medicine 2015. [press release] Retrieved from: https://www.nobelprize.org/nobel_prizes/medicine/laureates/2015/press.html

Norton, TR.; Bristonl, ML.; Read, GW.; Bushnell, OA.; Kashiwagi, M.; Okinaga, CM. and Oda, CS. (1973). Pharmacological evaluation of medicinal plants from Western Samoa. Journal of Pharmaceutical Sciences. 62(7): 1077-1082

O’Sullivan, D.; Green, L.; Stone, S.; Zareir, P.; Kharkrang, M.; Fong, D.; Connor, B. and La Flamme, AC. (2014). Treatment with the antipsychotic agent, risperidone, reduces disease severity in experimental autoimmune encephalomyelitis. PLoS One. 9(8): e104430

Omura, S. and Crump, A. (2004). The life and times of ivermectin - a success story. Nature Reviews Microbiology. 2(12): 984-989

Ondetti, MA.; Williams, NJ.; Sabo, EF.; Plluscec, J.; Weaver, ER. and Kocy, O. (1971). Angiotensinconverting enzyme inhibitors from the venom of Bothrops jararaca. Isolation, elucidation of structure and synthesis. Biochemistry. 10(22): 4033-4039

Paavilainen, HM. (2009). Medieval pharmacotherapy-Continuity and change: case studies from Ibn Sina and some of his late Medieval commentations. IDC Publishers: Netherlands

Padney, AV.; Joshi, SK.; Tekwani, BL. And Shauhan, VS. (1999). A colorimetric assay for heme in biological samples using 96-well plates. Analytical Biochemistry. 268(1): 159-161

Park, KC.; Wook, SK.; Yoo, YJ.; Wyndham, AM.; Baker, RT. and Chung, CH. (1997). Purification and characterization of BUP6, a new ubiquitin-specific protease in Saccharomyces cerevisiae. Archives of Biochemistry and Biophysics. 347(1): 78-84

Park, YK.; Koo, MH.; Ikegaki, M. and Contado, JL. (1997). Comparison of the flavonoid aglycone contents of Apis mellifera propolis from various regions of Brazil. Arq. Biol. Tecnol. 40: 97106

Parsons, AB.; Brost, RL.; Ding, H.; Li, Z.; Zhang, C.; Sheikh, B.; Brown, GW.; Kane, PM.; Hughes, TR. and Boone, C. (2004). Integration of chemical-genetic and genetic interaction data links bioactive compounds to cellular target pathways. Nature Biotechnology. 22(1): 62-69

Parsons, AB.; Lopez, A.; Givoni, IE.; Williams, DE.; Gray, CA.; Porter, J.; Chua, G.; Sopko, R.; Brostl, RL.; Ho, CH.; Wang, J.; Kertela, T.; Brenner, C.; Brill, JA.; Fernandez, GE.; Lorenz, TC.; Payne, GS.; Ishihara, S.; Ohya, Y.; Andrews, B.; Hughes, TR.; Frey, BJ.; Graham, TR.; Andersen, RJ. and 
Boone, C. (2006). Exploring the mode-of-action of bioactive compounds by chemical-genetic profiling in yeast. Cell. 126(3): 611-625

Patridge, E.; Gareiss, P.; Kinch, MS. and Hoyer, D. (2016). An analysis of FDA-approved drugs: natural products and their derivatives. Drug Discovery Today. 21(2): 204-207

Peterson, J.; Zheng, Y.; Bender, L.; Myers, A.; Cerione, R. and Bender, A. (1994). Interactions between the bud emergence proteins Bem1p and Bem2p and Rho-type GTPAses in yeast. Journal of Cell Biology. 127(5): 1395-1406

Philpott, CC. and Protchenko, O. (2008). Response to iron deprivation in Saccharomyces cerevisiae. Eukaryotic Cell. 7(1): 20-27

Philpott, CC.; Protchenko, O.; Kim, YW.; Boretsky, Y. and Shakoury-Elizeh, M. (2002). The response to iron deprivation in Saccharomyces cerevisiae: expression of siderophore-based systems of iron uptake. Biochemical Society Transactions. 30(4): 698-702

Pierce, SE.; Davis, RW.; Nislow, C. and Giaever, G. (2007). Genome-wide analysis of barcoded Saccharomyces cerevisiae gene-deletion mutants in pooled cultures. Nature Protocols. 2(11): 2958-2974

Piga, A.; Galanello, R.; Forni, GL.; Cappellini, MD.; Origa, R.; Zappu, A.; Donato, G.; Bordone, E.; Lavagetto, A.; Zanaboni, L.; Sechaud, R.; Hewson, N.; Ford, JM.; Opitz, H. and Alberti, D. (2006). Randomized Phase II trial of deferasirox (Exjade, ICL670), a once-daily, orallyadministered iron chelator, in comparison to deferoxamine in thalassemia patients with transfusional iron overload. Haemotologica. 91(7): 873-880

Piper, RC.; Cooper, AA.; Yang, H. and Stevens, TH. (1995). VPS27 controls vacuolar and endocytic traffic through a prevacuolar compartment in Saccharomyces cerevisiae. Journal of Cell Biology. 131(3): 603-617

Pitrowski, JS.; Simpkins, SW.; Li, SC.; Deshpande, R.; Mcllwain, ,SJ.; Ong, IM.; Myers, CL.; Boone, C and Andersen, RJ. (2015). Chemical genomic profiling via barcode sequencing to predict compound mode of action. Methods in Molecular Biology. 1263: 299-318

Planta, RJ. and Mager, WH. (1998). The list of cytoplasmic ribosomal p roteins of Saccharomyces cerevisiae. Yeast. 14(5): 471-477

Plumb, GW.; Pascual-Teresa, S.; Santos-Buelga, C.; Cheynier, V. and Williamson, G. (1998). Antioxidant properties of catechins and proanthocyanidins: Effect of polymerization, galloylation and glycosylation. Free Radical Research. 29(4): 351-358 
Poggiali, E.; Cassinerio, E.; Zanaboni, L. and Cappelini, MD. (2012). An update on iron chelation therapy. Blood Transfusion. 10(4): 411-422

Polevoda, B.; Span, L. and Sherman, F. (2006). The yeast translation release factors Mrf1p and Sup45p (eRF1) are methylated, respectively, by the methyltransferases Mtq1p and Mtq2p. Journal of Biological Chemistry. 281(5): 2562-2571

Porter, LJ.; Hrstich, LN. and Chan, BG. (1986). The conversion of procaynidins and prodelhinidins to canidin and delphinidin. Phytochemistry. 1: 223-230

Portnoy, ME.; Schmidt, PJ.; Rogers, RS. and Culotta, VC. (2001). Metal transporters that contribute copper to metallochaperones in Saccharomyces cerevisiae. Molecular Genetics and Genomics. 265(5): 873-882

Potterat, 0. and Hamburger, M. (2008). Drug discovery and development with plant-derived compounds. Progress in Drug Research. 65: 46-118

Prasad, T.; Chandra, A.; Mukhopadhyay, CK. and Prasad, R. (2006). Unexpected link between iron and drug resistance of Candida spp.: Iron depletion enhances membrane fluidity and drug diffusion, leading to drug-susceptible cells. Antimicrobial Agents and Chemotherapy. 50(11): 3597-3606

Protchenko, O.; Ferea, T.; Rashford, J.; Tiedeman, J.; Brown, PO.; Botstein, D. and Philpott, CC. (2001). Three cell wall mannoproteins facilitate the uptake of iron in Saccharomyces cerevisiae. Journal of Biological Chemistry. 276(52): 49244-49250

Pufahl, RA.; Singer, CP.; Peariso, KL.; Link, SJ.; Schmidt, PJ.; Fahrni, CJ.; Culotta, VC.; Penner-Hahn, JE. and O'Halloran, TV. (1997). Metal ion chaperone function of the soluble $\mathrm{Cu}(\mathrm{I})$ receptor Atx1p. Science. 278(5339): 853-856

Puig, S.; Askeland, E. and Thiele, DJ. (2005). Coordinated remodeling of cellular metabolism during iron deficiency through targeted mRNA degradation. Cell. 120(1): 99-110

Pujol, C.; Messer, SA.; Pfaller, M. and Soll, DR. (2003). Drug resistance is not directly affected by mating type locus zygosity in Candida albicans. Antimicrobial Agents and Chemotherapy. 47(4): 1207-1212

Quek, NC.; Matthews, JH.; Bloor, SJ.; Jones, DA.; Bircham, PW.; Heathcott, RW. and Atkinson, PH. (2013). The novel equisetin-like compound, TA-289, causes aberrant mitochondrial morphology which is independent of the production of reactive oxygen species in Saccharomyces cerevisiae. Molecular Biosystems. 9(8): 2125-2133 
Quiroga, EN.; Sampietro, AR. and Vattuone, MA. (2001). Screening antifungal activities of selected medicinal plants. Journal of Ethnopharmacology. 74(1): 89-96

Raskin, I.; Ribnicky, DM.; Komarnytsky, S.; Ilic, N.; Poulev, A.; Borisjuk, N.; Brinker, A.; Moreno, DA.; Ripoll, C.; Yakoby, N.; O’Neal, JM.; Cornwell, T.; Pastor, I.; Fridlender, B. (2002). Plants and human health in the twenty-first century. Trends in Biotechnology. 20(12): 522-531

Rauha, JP.; Remes, S.; Heinonen, M.; Hopia, A.; Kahkonen, M.; Kujala, T.; Pihlaja, K.; Vuorela, H. and Vuorela, P. (2000). Antimicrobial effects of Finnish plant extracts containing flavonoids and other phenolic compounds. International Journal of Food Microbiology. 56(1): 3-12

Reddy, L.; Odhav, B.; and Bhoola, KD. (2003). Natural products for cancer prevention: a global perspective. Pharmacology \& Therapeutics. 99(1): 1-13

Rispail, N.; Dita, MA.; Gonzales-Verdeho, C.; Perez-de-Lugue, A.; Castillejo, MA.; Prats, E.; Roman, B.; Jorrin J.; Rubiales, D. (2007). Plant resistance to parasitic plants: molecular approaches to an old foe. New Phytologist. 173(4): 703-712

Robinson, JS.; Klionsky, DJ.; Banta, LM. and Emr, SD. (1988). Protein sorting in Saccharomyces cerevisiae: isolation of mutants defective in the delivery and processing of multiple vacuolar hydrolases. Molecular \& Cellular Biology. 8(11): 4936-4948

Robinson, DG.; Chen, W.; Stoery, JD. and Gresham, D. (2014). Design and analysis of Bar-seq experiments. G3. 4(1): 11-18

Roncoroni, M.; Santiago, M.; Hooks, DO.; Moroney, S.; Harsch, MJ.; Lee, SA.; Richards, KD.; Nicolau, L. and Gardner, RC. (2011). The yeast IRC7 gene encodes a $\beta$-lyase responsible for production of the varietal thiol 4-mercapto-4-methylpentan-2-one in wine. Food Microbiology. 28(5): 926-935

Rothman, JH. and Stevens, TH. (1986). Protein sorting in yeast: mutants defective in vacuole biogenesis mislocalize vacuolar proteins into the late secretory pathway. Cell. 47(6): 10411051

Rowinsky, EK. And Donehower, RC.(1995). Paclitaxel (taxol). New England Journal of Medicine. 332(15): 1004-1014

Sadowska-Bartosz, I.; Paczka, A.; Molon, M. and Bartosz, G. (2013). Dimethyl sulfoxide induces oxidative stress in the yeast Saccharomyces cerevisiae. FEMS Yeast Research. 13(8): 820830 
Saliou, C.; Rimbach, G.; Moini, H. (2001). Solar ultraviolet-induced erythema in human skin and nuclear-factor-kappa-B-dependent gene expression in keratinocytes are moedulated by a French maritime pine bark extract. Free Radical and Biology Medicine. 30: 154-160

Satish, S.; Mohana, DC.; Raghavendra, MP. and Raveesha, KA. (2007). Antifungal activity of some plant extracts against important seed borne pathogens of Aspergillus sp. Journal of Agricultural Technology. 3(1): 109-119

Saveanu, C.; Fromon-Racine, M.; Harington, A.; Richard, F.; Namane, A. and Jacquier, A. (2001). Identification of 12 new yeast mitochondrial ribosomal proteins including 6 that have no prokaryotic homologues. Journal of Biological Chemistry. 276(19): 15861-15867

Scalbert, A. (1991). Antimicrobial properties of tannins. Phytochemistry. 30(12): 3875-3883

Schewe, T.; Sadik, C.; Klotz, LOL.; Yoshimoto, T.; Kuhn, H. and Sies, H. (2001). Polyphenols in cocoa: inhibition of mammalian 15-lipoxygenase. Biological Chemistry. 382(12): 1687-1696

Schmedtje, JF Jr.; Ji, YS.; Liu, WL.; DuBois, RN. and Runge, MS. (1997). Hypoxia induces cyclooxygenase-2 via the NF-kappaB p65 transcription factor in human vascular endothelial cells. Journal of Biological Chemistry. 272(1): 601-608

Schneider-Poetsch, T.; Ju, J.; Eyler, DE.; Dang, Y.; Bhat, S.; Merrick, WC.; Green, R.; Shen, B. and Liu, J0. (2010). Inhibition of eukaryotic translation elongation by cycloheximide and lactimidomycin. Nature Chemical Biology. 6(3): 209-217

Schofield, P.; Mbugua, DM. and Pell, AN. (2001). Analysis of condensed tannins: a review. Animal Feed Science and Technology. 91: 21-40

Shahat, AA.; Cos, P.; De Bruyne, T.; Apers, S.; Hammouda, FM.; Ismail, SI.; Azzam, S.; Claeys, M.; Goovaerts, E.; Pieters, L.; Vanden Berghe, D. and Vlietinck, AJ. (2002). Antiviral and antioxidant activityof flavonoids and proanthocyanidins from Crataegus sinaica. Planta Medica. 68(6): 539-541

Shakoury-Elizeh, M.; Tiedeman, J.; Rashford, J.; Ferea, T.; Demeter, J.; Garcia, E.; Rolfes,R.; Brown, PO.; Botstein, D.; and Philpott, CC. (2004). Transcriptional remodeling in response to iron deprivation in Saccharomyces cerevisiae. Molecular Biology of the Cell. 15(3): 1233-1243

Shakoury-Elizeh, M.; Protchenko, O.; Berger, A.; Cox, K.; Gable, K.; Dunn, TM.; Prinz, WA.; Bard, M. and Phillpott, CC. (2010). Metabolic response to iron deficiency in Saccharomyces cerevisiae. Journal of Biological Chemistry. 285(19): 14823-14833

Shao, ZH.; Wojcik, KR.; Dossumbekova, A.; Hsu, C.; Mehendale, SR.; Li, CW.; Qin, Y.,; Sharp, WW.; Chang, WT.; Hamann, KJ.; Yuan, CS. and vanden Hoek, TL. (2009). Grape seed 
proanthocyanidins protect cardiomyocytes from ischemia and reperfusion injury via AktNOS signaling. Journal of Cellular Biochemistry. 107(4): 697-705

Shellard, EJ.; (2000). Alkaloids from snowdrops. The Pharmaceutical Journal. 264: 883

Singh, AJ.; Xu, CX.; Xu, X.; West, LM.; Wilmes, A.; Chan, A.; Hamel, E.; Miller, JH.; Northcote, PT. and Ghosh, AK. (2010). Peloruside B, a potent antitumor macrolide from the New Zealand marine sponge Mycale hentscheli: isolation, structure, total synthesis, and bioactivity. Journal of Organic Chemistry. 75(1): 2-10

Siriwardana, G. and Seligman, PA. (2013). Two cell cycle blocks caused by iron chelation of neuroblastoma cells: separating cell cycle events associated with each block. Physiological Reports. 1(7): e00176

Smith, AM.; Heisler, LE.; Mellor, J.; Kaper, F.; Thomson, MJ.; Chee, M.; Roth, FP.; Giaever, G. and Nislow, C. (2009). Quantitative phenotyping via deep barcode sequencing. Genome Research. 19(10): 1836-1842

Smith, AM.; Ammar, R.; Nislow, C. and Giaever, G. (2010). A survey of yeast genomic assays for drug and target discovery. Pharmacology \& Therapeutics. 127(2): 156-164

Smith, AM.; Durbic, T.; Kittanakom, S.; Giaever, G. and Nislow, C. (2012). Barcode sequencing for understanding drug-gene interactions. Methods in Molecular Biology. 910: 55-69.

Sofic, E.; Riederer, P.; Heinsen, H.; Beckmann, H.; Reynolds, GP.; Hebenstreit, G. and Youdin, MBH. (1988). Increased iron (III) and total iron content in post mortem substantia nigra of parkinsonian brain. Journal of Neural Transmission. 74: 199-205

South, PK. and Miller, DD. (1998). Iron binding by tannic acid: Effects of selected ligans. Food Chemistry 63(2): 167-172

Stearman, R.; Yuan, DS.; Yamaguchi-Iwai, Y.; Klausner, RD. and Dancis, A. (1996). A permeaseoxidase complex involved in high-affinity iron uptake in yeast. Science. 271(5255): 15521557

Stolc, V. and Altman, S. (1997). Rpp1, an essential protein subunit of nuclear RNase P required for processing of precursor tRNA and 35S precursor rRNA in Saccharomyces cerevisiae. Genes \& Development. 11(21): 2926-2937

Sugiyama, M. and Nikawa, J. (2001). The Saccharomyces cerevisiae Isw2p-Itc1p complex represses IN01 expression and maintains cell morphology. Journal of Bacteriology. 183(17): 49854993 
Szczypka, MS.; Zhu, Z.; Silar, P.; Thiele, DJ. (1997). Saccharomyces cerevisiae mutants altered in vacuole function are defective in copper detoxification and iron-responsive gene transcription. Yeast. 13(15): 1423-1435

Tamarit, J.; Irazusta, V.; Moreno-Cermeno, A. and Ros, J. (2006). Colorimetric assay for the quantitation of iron in yeast. Analytical Biochemistry. 351(1): 149-151.

Tan, S. and Liu ZP. (2015). Natural products as zinc-dependent histone deacetylase inhibitors. ChemMedChem. 10(3): 441-450

Tanji, K.; Imaizumi, T.; Matsumiya, T.; Itaya, H.; Fujimoto, K.; Cui, XF.; Toki, T.; Ito, E.; Yoshida, H.; Wakabayashi, K. and Satoh, K. (2001). Desferrioxamine, an iron chelator, upregulates cyclooxygenase-2 expression and prostaglandin production in a human macrophage cell line. Biochimica et Biophysica Acta. 150(2-3): 227-235

Tao, L.; Zhu, F.; Qin, C.; Zhang, C.; Xu, F.; Tan, CY.; Jiang, YY. and Chen, YZ. (2014). Nature's contribution to today's pharmacopoeia. Nature Biotechnology. 32(10): 979-980

Terkeltaub, RA. (2009). Colchicine update: 2008. Seminars in Arthritis and Rheumatism. 38(6): 411419

Tkach, JM.; Wimit, A.; Lee, AY.; Riffle, M.; Costanzo, M.; Jaschob, D.; Hendry, JA.; Ou, J.; Moffat, J.; Boone, C.; Davis, TN.; Nislow, C. and Brown, GW. (2012). Dissecting DNA damage response pathways by analyzing protein localization and abundance changes during DNA replication stress. Nature: Cell Biology. 14(9): 966-976

Tong, AH.; Evangelista, M.; Parsons, AB.; Xu, H.; Bader, GD.; Page, N.; Roinson, M.; Raghiizadeh, S.; Hogue, CW.; Bussey, H.; Andrews, B.; Tyers, M.; Boone, C. (2001). Systematic genetic analysis with ordered arrays of yeast deletion mutants. Science. 294(5550): 2364-2368

Towle, MJ.; Salvato, KA.; Budrow, J.; Wels, BF.; Kuznetsov, G.; Aalfs, KK.; Welsh, S.; Zheng, W.; Seletsky, BM.; Palme, MH.; Bahgood, GJ.; Singer, LA.; Dipietro, LV.; Wange, YC.; Quincy, DA.; Davis, A.; Yoshimatsu, K.; Kishi, Y.; Yu, MJ. and Littlefield, BA. (2001). In vitro and in vivo anticancer activities of synthetic macrocyclic ketone analogues of halichondrin B. Cancer Research. 61(3): 1013-1021

Tu, Y. (2011). The discovery of artemisinin (qingshaosu) and gifts from Chinese medicine. Nature Medicine. 17: 1217-1220

Turi, TG. and Loper, JC. (1992). Multiple regulatory elements control expression of the gene encoding the Saccharomyces cerevisiae cytochrome P450, lanosterol 14 alpha-demethylase (ERG11). Journal of Biological Chemistry. 267(3): 2046-2056 
Uhe, G. (1974). Medicinal plants of Samoa: A preliminary survey of the use of plants for medicinal purposes in the Samoan Islands. Economic Botany. 28(1): 1-30

van Dyke, N.; Baby, J. and van Dyke, MW. (2006). Stm1p, a ribosome-associated protein, is important for protein synthesis in Saccharomyces cerevisiae under nutritional stress conditions. Journal of Molecular Biology. 358(4): 1023-1031

van Ho, A.; Ward, DM. and Kaplan, J. (2002). Transitional metal transport in yeast. Annual Reviews in Microbiology. 56: 237-261

Wade, D.; Andreu, D.; Mitchell, SA.; Silveira, AM.; Boman, A.; Boman, HG. and Merrifield, RB. (1992). Antibacterial peptides designed as analogs or hybrids of cecropins and melittin. International Journal of Protein Research. 40(5): 429-436

Wagih, O. \& Parts, L. (2014). Gitter: A robust and accurate method for quantification of colony sizes from plate images. G3. 4(3): 547-552

Walsh, P.; Bursac, D.; Law, YC.; Cyr, D. and Lithgow, T. (2004). The J-protein family: modulating protein assembly, disassembly and translocation. EMBO Reports. 5(6): 567-571

Wang, Q. and Chang, A. (1999). Eps1, a novel PDI-related protein involved in ER quality control in yeast. EMBO Journal. 18(21): 5972-5982

Waters, BM. and Eide, DJ. (2002). Combinatorial control of yeast FET4 gene expression by iron, zinc and oxygen. Journal of Biological Chemistry. 277(37): 33749-33757

Webster, D.; Taschereau, P.; Belland, RJ.; Sand, C. and Rennie, RP. (2008). Antifungal activity of medicinal plant extracts; preliminary screening studies. Journal of Ethnopharmacology. 115: 140-146

Wessecker, S.; Engel, K.; Simon-Haarhaus, B.; Wittmer, A.; Pelz, K.; Schempp, CMS. (2007). Screening of plant extract for antimicrobial activity against bacteria and yeast with dermatological relevance. Phytomedicine. 14(7-8): 508-516

Wessling-Resnick, M. (2010). Iron homeostasis and the inflammatory response. Annual Review of Nutrition. 21: 105-122

West, LM.; Northcote, PT.; Hood, KA.; Miller, JH and Page, MJ. (2000). Mycalamide, D, a new cytotoxic amide from the New Zealand marine sponge Mycale species. Journal of Natural Products. 63: 707-709

Whistler, AW. (1996). Samoan Herbal Medicine. Isle Botanica, Honolulu: Hawaii, print. 
Whistler, AW. (2000). Plants in Samoa Culture: the ethnobotany of Samoa. Isle Botanica, Honolulu: Hawaii, print.

World Health Organization (1998). Medicinal plants in the South Pacific. WHO Regional Publications, Western Pacific Series no. 19, WHO Regional Office for the Western Pacific, Manila.

Wichmann, H.; Hengst, L. and Gallwitz, D. (1992). Endocytosis in yeast: evidence for the involvement of a small GTP-binding protein (Ypt7p). Cell. 71(7): 1131-1142

Williams, SA.; Chen, LF.; Kwon, H.; Fenard, D.; Bisgrove, D.; Verdin, E and Greene, WC. (2004). Prostratin antagonizes HIV latency by activating NF-kB. Journal of Biological Chemistry. 279(40): 42008-42017

Wilmes, A.; Hanna, R.; Heathcott, RW.; Northcote, PT.; Atkinson, PH; Bellows, DS. and Miller, JH. (2012). Chemical genetic profiling of the microtubule-targeting agent pelorudise $A$ in budding yeast Saccharomyces cerevisiae. Gene. 497(2): 140-146

Wilson, CL.; Solar, JM.; Ghaouth, AE. and Wisniewski, ME. (1997). Rapid evaluation of plant extracts and essential oilds for antifungal activity against Botrytis cinerea. Plant Disease. 81(2): 204210

Winzeler, EA.; Shoemaker, DD.; Astromoff, A.; LIanh, H.; Anderson, K.; Andre, B.; Bangham, R.; Benito, R.; Boeke, JD.; Bussey, H.; Chu, AM.; Connelly, C.; Davis, K.; Dietrich, F.; Dow, SW.; El Bakkoury, M.; Foury, F.; Friend, SH.; Gentalen, E.; Giaever, G.; Hegemann, JH.; Jones, T.; Laub, M.; Liao, H.; Davis, RW.; Liedbundguth, N.; Lockhart, DJ.; Lucau-Danila, A.; Lussier, M.; M'Rabet, N.; Menard, P.; Mittmann, M.; Pai, C.; Rebischung, C.; Revuelta, JL.; Riles, L.; Roberts, CJ.; Ross-MacDonald, P.; Scherens, B.; Snyder, M.; Sookhai-Mahadeo, S.; Storms, RK.; Veronneau, S.; Voet, M.; Volckaert, G.; Ward, TR.; Wysocki, R.; Yen, GS.; Yu, K.; Zimmermann, K.; Phillippsen, P.; Johnston, M. and Davis, RW. (1999). Functional characterization of the S. cerevisiae genome by gene deletion and parallel analysis. Science. 285(5429): 901-906

Wu, J.; Carmen, AA.; Kobayashi, R.; Suka, N. and Grunstein, M. (2001). HDA2 and HDA3 are related p roteins that interact with and are essential for the activityof the yeast histone deacetylase HDA1. Proceedings of the National Academy of Science. 98(8): 4391-4396

Xiong, S.; She, H.; Sung, CK and Tsukamoto, H. (2003). Iron-dependent activation of NF-kB in Kupffer cells: a priming mechanism for alcoholic liver disease. Alcohol: An International Biomedical Journal. 30(2): 107-113

Xu, D.; Sillaots, S.; Davison, J.; Hu, W.; Jiang, B.; Kauffman, S.; Martel, N.; Ocampo, P.; Oh, C.; Trosok, S.; Veillette, K.; Wang, H.; Yang, M.; Zhang, L.; Becker, J.; Martin, CE. and Roemer, T. (2009). Chemical genetic profiling and characterization of small-molecule compounds that affect the 
biosynthesis of unsaturated fatty acids in Candida albicans. Journal of Biological Chemistry. 284(29): 19754-19764

Xu, N.; Cheng, X.; Yu, Q.; Zhang, B.; Ding, X.; Xing, L. and Li, M. (2012). Identification and functional characterization of mitochondrial carrier Mrs4 in Candida albicans. FEMS Yeast Research. 12(7): 844-858

Yamaguchi-Iwai, Y.; Stearman, R.; Dancis, A. and Klausner, RD. (1996). Iron-regulated DNA binding by the AFT1 protein controls the iron regulon in yeast. EMBO Journal. 15(13): 3377-3384

Yan, Z.; Costanzo, M.; Heisler, LE.; Paw, J.; Kaper, F.; Andrews, BJ.; Boone, C.; Giaever, G. and Nislow, C. (2008). Yeast Barcoders: a chemogenomic application of a universal donor-strain collection carrying bar-code identifies. Nature Methods. 5(8): 719-725

Yang, H.; Bard, M.; Bruner, DA.; Gleeson, A.; Deckelbaum, RJ.; Aljinovic, G.; Pohl, TM.; Rothstein, R. and Sturley, SL. (1996). Sterol esterification in yeast: a two-gene process. Science. 272(5266): 1353-1356

Yang, CS.; Yang, GY.; Landau, JM.; Kim, S. and Liao, J. (1998). Tea and tea polyphenols inhibit cell hyperproliferation, lung tumorigenesis and tumor progression. Experimental Lung Research. 24(4): 629-639

Yarunin, A.; Panse, VG.; Petfalski, E.; Dez, C.; Tollervey, D. and Hurt, EC. (2005). Functional link between ribosome formation and biogenesis of iron-sulfur proteins. EMBO Journal. 24(3): 580-588

Yesilada, E.; Tsuchiya, K.; Takaishi, Y. and Kawazoe, K. (2000). Isolation and characterization of free radical scavenging flavonoi glycosides from the flowers of Spartium junceum by activityguided fractionation. Journal of Ethnopharmacology 73: 471-478

Yibmantasiri, P.; Leahy, DC.; Busby, BP.; Angemayr, SA.; Sorgo, AG.; Boeger, K.; Heathcott, R.; Barber, JM.; Matthews, JH.; Northcote, PT.; Atkinson, PH. and Bellows, DS. (2012). Molecular basis for fungicidal action of neothyonidioside, a triterpene glycoside from the sea cucumber, Australostichopus mollis. Molecular Biosystems. 8(3): 902-912

Yokozawa, T.; Chen, CP.; Dong, E.; Tanaka, T.; Nonaka, GI. and Nishioka, I. (1998). Study on the inhibitory effect of tannins and flavonoids against the 1,1-diphenyl-2 picrylhydrazyl radical. Biochemical Pharmacology. 56(2): 213-222

Yompakdee, C.; Ogawa, N.; Harashima, S. and Oshima, Y. (1996). A putative membrane protein, Pho88p, involved in inorganic phosphate transport in Saccharomyces cerevisiae. Molecular \& General Genetics. 251(5): 580-590 
Yoneda, S. and Nakatsubo, F. (1998). Effects of the hydroxylation patterns and degrees of polymerization of condensed tannins on their metal-chelating capacity. Journal of Wood Chemistry and Technology. 18(2): 193-205

Yoshida, A.; Yoshino, F.; Tsubata, M.; Ikeguchi, M.; Nakamura, T. and Lee, MC. (2011). Direct assessment by electron spin resonance spectroscopy of the antioxidant effects of French maritime pine bark extract in the maxillofacial region of hairless mice. Journal of Clinical Biochemical Nutrition. 49(2): 79-86

Youdell, ML.; Kizer, KO.; Kisseleva-Romanova, E.; Fuchs, SM.; Duro, E.; Strahl, BD. and Mellor, J. (2008). Roles for Ctk1 and Spt6 in regulating the different methylation states of histone H3 lysin 36. Molecular and Cellular Biology. 28(16): 4915-4926

Yu, C.; Kennedy, NJ.; Chang, CCY. and Rothblatt, JA. (1996). Molecular cloning and characterization of two isoforms of Saccharomyces cerevisiae acyl-CoA:sterol acyltransferase. Journal of Biological Chemistry. 271(39): 24157-24163

Yuan, DS.; Dancis, A.; Klausner, RD. (1997). Restriction of copper export in Saccharomyces cerevisiae to a late Golgio or post-Golgi compartment in the secretory pathway. Journal of Biological Chemistry. 272(41): 25787-25793

Zakharova, M. and Ziegler, HK. (2005). Paradoxical anti-inflammatory actions of TNF-alpha: inhibnition of IL-12 and IL-23 via TNF receptor 1 in macrophages and dendritic cells. Journal of Immunology. 175(8): 5024-5033

Zhang, T.; Lei, J.; Yang, J.; Xy, K.; Wang, R. and Zhang, Z. (2011). An improved method for whole protein extraction from yeast Saccharomyces cerevisiae. Yeast. 28(11): 795-798

Zhang, C.; and Zhang, F. (2014). Iron homeostasis and tumorigenesis: molecular mechanisms and therapeutic opportunities. Protein \& Cell. 6(2): 88-100

Zhao, S.; Douglas, NW.; Heine, MJ.; Williams, GM.; Winther-Larse, HC. and Meaden, PG. (1994). The STL1 gene of Saccharomyces cerevisiae is predicted to encode a sugar transporter-like protein. Gene. 146(2): 215-219

Zhao, X.; Muller, EG. and Rothstein, R. (1998). A suppressor of two essential checkpoint genes identifies a novel protein that negatively affects dNTP pools. Molecular Cell. 2(3): 329-340 


\section{Appendix I: Media}

1. Yeast-Extract Peptone Dextrose (YPD) Media:

\begin{tabular}{cc} 
Ingredient & Weight $(\mathrm{w} / \mathrm{v})$ \\
\hline Yeast Extract & $1 \%$ \\
Bacto-Peptone & $2 \%$ \\
Adenine & $0.012 \%$ \\
Glucose & $2 \%$ \\
Agar & $2 \%$
\end{tabular}

2. Synthetic Complete Media

\begin{tabular}{cc} 
Ingredient & Weight (w/v) \\
\hline Yeast nitrogen base (without amino acids or ammonium sulfate) & $1 \%$ \\
Monosodium glutamate & $0.17 \%$ \\
Amino acid mixture & $0 / 2 \%$ \\
Glucose & $2 \%$ \\
Agar & $2 \%$
\end{tabular}

Amino Acid Mixture:

$3 \mathrm{~g}$ adenine, $2 \mathrm{~g}$ uracil, $2 \mathrm{~g}$ inositol, $0.2 \mathrm{~g}$ para-aminobenzoic acid, $2 \mathrm{~g}$ alanine, $2 \mathrm{~g}$ arginine, $2 \mathrm{~g}$ asparagines, 2 g aspartic acid, 2 g cysteine, 2 g glutamic acid, 2 g glutamine, 2 g glycine, $2 \mathrm{~g}$ histidine, $2 \mathrm{~g}$ isoleucine, $10 \mathrm{~g}$ leucine, $2 \mathrm{~g}$ lysine, $2 \mathrm{~g}$ methionine, $2 \mathrm{~g}$ phenylalanine, $2 \mathrm{~g}$ proline, 2 g serine, 2 g threonine, $2 \mathrm{~g}$ tyrosine, $2 \mathrm{~g}$ tryptophan, $2 \mathrm{~g}$ valine 


\section{Appendix II: Bioactivity at $70^{\circ} \mathrm{C}$}
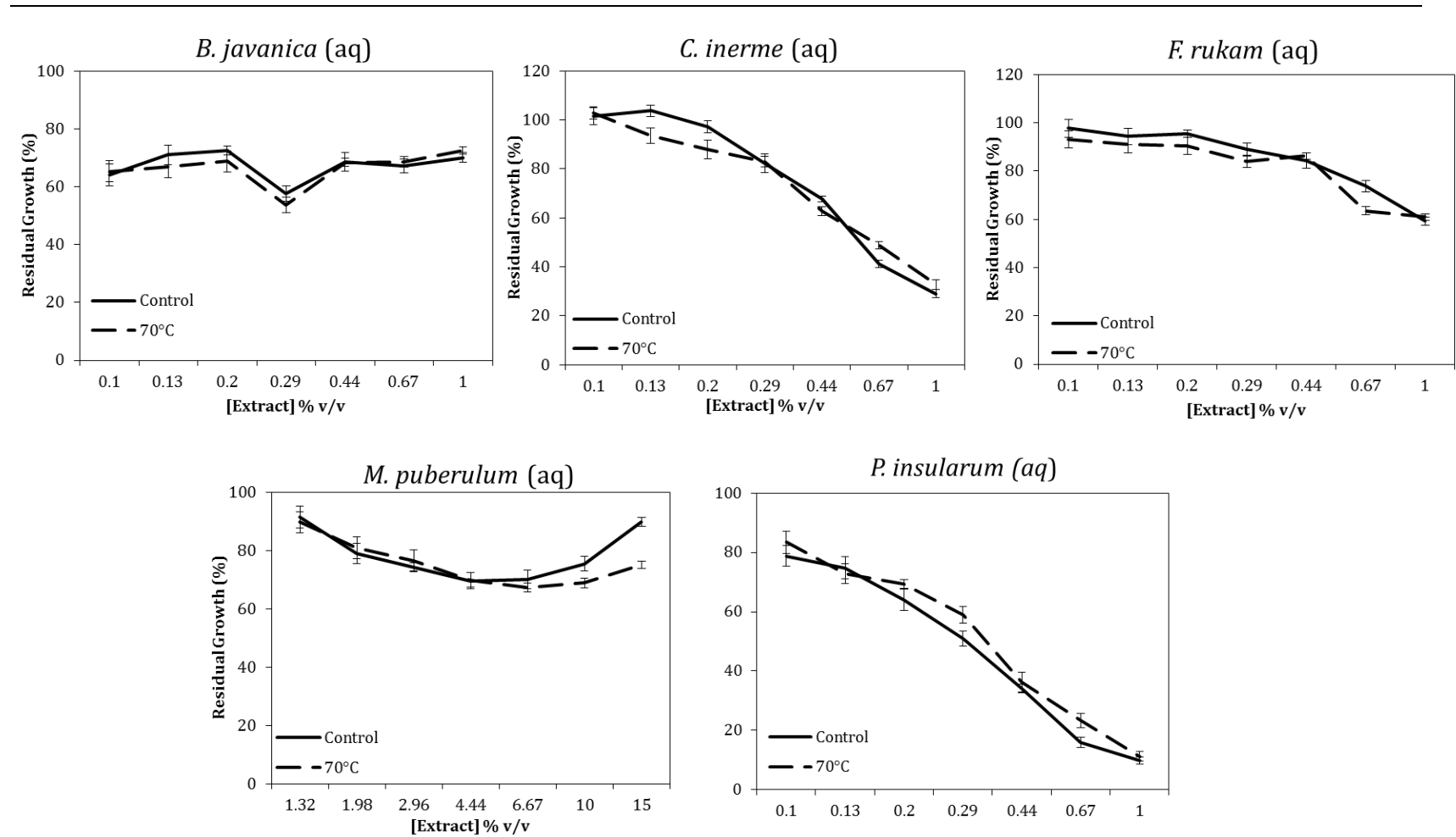

Figure A2: Bioactivity of the five bioactive aqueous extracts after heating at $70^{\circ} \mathrm{C}$. Subsamples of the extracts were aliquot into Eppendorf tubes and heated at $70^{\circ} \mathrm{C}$ overnight, before cooling to RT and serial diluted. WT yeast were inoculated at $5 \times 10^{5}$ cells $/ \mathrm{mL}$ and grown in increasing concentrations of the heated extracts (dashed black lines, labelled $70^{\circ} \mathrm{C}$ ), and their growth was compared to subsamples of the same extract stored at $-20^{\circ} \mathrm{C}$ overnight (solid black lines, Control). Bioactivity of WT yeast in the aqueous extracts of A: B. javanica. B: C. inerme. C: F. rukam. D: M. puberulum. E: P. insularum. 


\section{Appendix III: Genotypes of Strains Used in this Thesis}

All yeast strains used in this thesis are derived from S288c and maintained at $-80^{\circ} \mathrm{C}$ as glycerol stocks.

\begin{tabular}{clll} 
Strain & Name & Genotype & Reference \\
\hline Y7092 & WT & MAT $\alpha$; can1 $\Delta:$ :STE2pr-Sp_his5; lyp1 $\Delta ;$ his3 $\Delta 1$ & Starting strain from \\
& & leu2 $\Delta 0$ ura3 $\Delta 0$ met15 $\Delta 0$ LYS2+ & Boone Lab \\
BY4741 & WT & MATa; his3 $\Delta 0$ leu2 $\Delta 0$ met15 $\Delta 0$ ura3 $\Delta 0$ & Biosystems \\
BY4742 & WT & MAT $\alpha$, his3 $\Delta 1$ leu2 $\Delta 0$ lys2 $\Delta 0$ ura3 $\Delta 0$ & Biosystems \\
BY4743 & WT & BY4741 x BY4742 & Biosystems
\end{tabular}

GFP strains with RedStar2 MAT $\alpha$; can1 $1 \Delta:$ STE2pr-URA3; lyp1 $\triangle::$ mCherry- $\quad$ Bircham, P (2014)

and mCherry Nat; his3 $\Delta$ a; leu2 $\Delta 0$; ura3 $\Delta 0:: N L S-R e d S t a r 2-$

$\mathrm{HPH}$; LYS2+ and mated to the GFP Collection

(BioSystems)

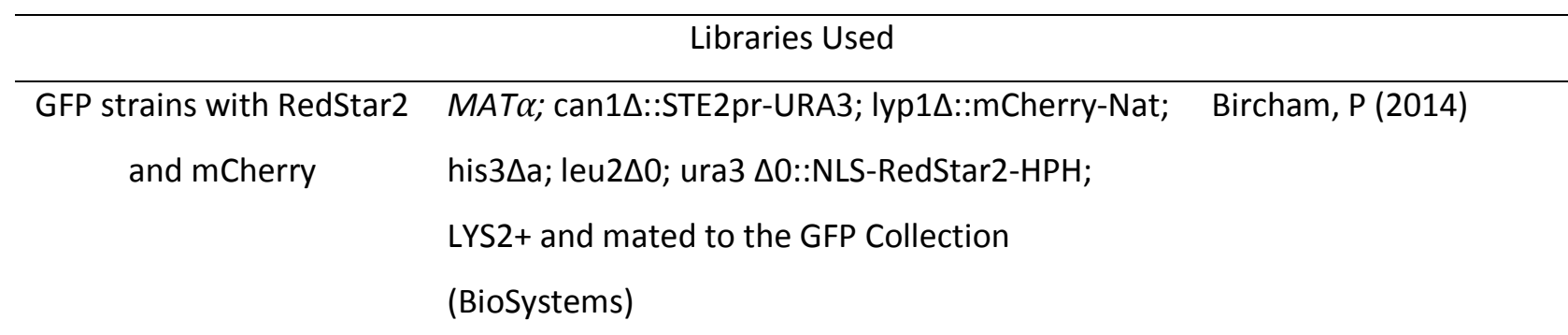

Haploid MATa deletion

Boone Lab

mutant array

Diploid Heterozygous

Biosystems

Library

Diploid Homozygous

Biosystems

Library 


\section{Appendix IV: 128 Mutants from Agar-Based Screen}

\begin{tabular}{|c|c|c|c|c|c|c|}
\hline \multicolumn{2}{|c|}{ Replicate 1} & \multicolumn{2}{|c|}{ Replicate 2} & \multicolumn{2}{|c|}{ Replicate 3} & \multirow{2}{*}{$\begin{array}{l}\text { Combined } \\
\text { Unique }\end{array}$} \\
\hline p-value & Gene & p-value & Gene & p-value & Gene & \\
\hline 0.00857 & AIF1 & 0.01233 & ADE5,7 & 0.01305 & ADE5,7 & ADE5,7 \\
\hline 0.01292 & ARG3 & 0.01833 & ANT1 & 0.02313 & AIF1 & AIF1 \\
\hline 0.03925 & ARO3 & 0.01733 & AR07 & 0.04954 & ANT1 & ANT1 \\
\hline 0.00854 & AR07 & 0.00109 & BEM1 & 0.02185 & ARG3 & ARG3 \\
\hline 0.00325 & BEM4 & 0.0028 & BEM4 & 0.0411 & ARO3 & ARO3 \\
\hline 0.02948 & BUB3 & 0.01229 & CAR1 & 0.04114 & BEM1 & AR07 \\
\hline 0.0477 & BUD7 & 0.01689 & CBC2 & 0.02041 & BUB3 & BEM1 \\
\hline 0.02 & CBC2 & $4.22 \mathrm{E}-06$ & $\mathrm{CHO} 2$ & 0.04793 & BUD7 & BEM4 \\
\hline $5.5 \mathrm{E}-06$ & $\mathrm{CHO} 2$ & 0.02872 & CIK1 & 0.03811 & CAR1 & BUB3 \\
\hline 0.00085 & CNE1 & 0.01327 & CMK2 & 0.00226 & CBC2 & BUD7 \\
\hline 0.00166 & COX10 & 0.01357 & CNE1 & $3.5 \mathrm{E}-08$ & CHO2 & CAR1 \\
\hline 0.01695 & СРA2 & $6.30 \mathrm{E}-06$ & CoX10 & 0.0172 & CIK1 & СBC2 \\
\hline 0.02398 & CPA2 & $7.62 \mathrm{E}-08$ & СРA2 & 0.03695 & CMK2 & $\mathrm{CHO} 2$ \\
\hline 0.03022 & CSF1 & 0.03209 & CSF1 & 0.00584 & CNE1 & CIK1 \\
\hline 0.00427 & CSH1 & 0.00592 & CTF19 & 0.00193 & CoX10 & CMK2 \\
\hline 0.02999 & CUE3 & 0.01909 & CUE3 & 0.00752 & СРA2 & CNE1 \\
\hline 0.00607 & DAL1 & 0.0013 & DDR48 & 0.0304 & CSH1 & COX10 \\
\hline 0.00613 & DDR48 & 0.00969 & ERP6 & 0.0169 & CTF19 & СРА2 \\
\hline 0.04756 & ERP6 & 0.04449 & FKH2 & 0.00603 & DAL1 & CSF1 \\
\hline 0.03747 & FKH2 & 0.01493 & FZF1 & 0.01425 & DDR48 & CSH1 \\
\hline 0.00998 & FZF1 & 0.04381 & GEM1 & 0.02929 & ERP6 & CTF19 \\
\hline $3.8 \mathrm{E}-05$ & GSH2 & 0.02898 & GPG1 & 0.02103 & GEM1 & CUE3 \\
\hline 0.01683 & GUP1 & 0.04958 & GSH2 & 0.03436 & GPG1 & DAL1 \\
\hline 0.04171 & HAT1 & 0.02748 & GYP1 & 0.00812 & GSH2 & DDR48 \\
\hline 0.04706 & IDH1 & 0.04955 & HAT1 & 0.03107 & GUP1 & ERP6 \\
\hline 0.0125 & INO2 & 0.04276 & IPK1 & 0.03141 & GYP1 & FKH2 \\
\hline 0.03005 & IPK1 & 0.00826 & ITT1 & 0.01754 & IDH1 & FZF1 \\
\hline 0.04336 & KAR3 & 0.0465 & KAP122 & 0.02762 & INO2 & GEM1 \\
\hline 0.02958 & KNS1 & 0.0269 & KNS1 & 0.02413 & IPK1 & GPG1 \\
\hline 0.00741 & LYS1 & 0.01553 & LYS12 & 0.008 & ITT1 & GSH2 \\
\hline 0.00208 & LYS12 & 0.03222 & MET10 & 0.03748 & KAP122 & GUP1 \\
\hline 0.02378 & NRK1 & 0.00057 & NRK1 & 0.00311 & KAR3 & GYP1 \\
\hline 0.02889 & NUP60 & 0.01654 & NUP60 & 0.03422 & KNS1 & HAT1 \\
\hline 8E-07 & OPI3 & 7.61E-06 & OPI3 & 0.00154 & LYS1 & IDH1 \\
\hline 0.03521 & OSH6 & 0.02464 & OSH6 & 0.03296 & MET10 & INO2 \\
\hline 0.0148 & OST3 & 0.01998 & OST3 & 0.00012 & OPI3 & IPK1 \\
\hline 0.01776 & PAC10 & 0.01884 & PAA1 & 0.0292 & OST3 & ITT1 \\
\hline 0.01503 & PDC6 & 0.03422 & PDC6 & 0.02689 & PAA1 & KAP122 \\
\hline 0.01995 & PNT1 & 0.00643 & PH086 & 0.02545 & PAC10 & KAR3 \\
\hline 0.03209 & RIM8 & 0.03064 & PNT1 & 0.04876 & PDC6 & KNS1 \\
\hline 0.02146 & RPL11B & 0.005 & REC8 & 0.00699 & PH086 & LYS1 \\
\hline 0.04292 & RPL16B & 0.0377 & RIM8 & 0.046 & REC8 & LYS12 \\
\hline 0.00919 & RPL37B & 0.00382 & R0X1 & 0.01277 & R0X1 & MET10 \\
\hline 0.00857 & RPL6B & 0.02055 & RPA34 & 0.01023 & RPA34 & NRK1 \\
\hline 0.00077 & RPL9B & 0.00819 & RPL11B & 0.00193 & RPL11B & NUP60 \\
\hline 0.00491 & SET6 & 0.03323 & RPL37B & 0.00225 & RPL16B & OPI3 \\
\hline
\end{tabular}




\begin{tabular}{|c|c|c|c|c|c|c|}
\hline 0.00739 & SK01 & 0.00973 & RPL6B & 0.00643 & RPL6B & OSH6 \\
\hline $3.8 \mathrm{E}-05$ & SLG1 & 8.51E-06 & RPL9B & 0.03652 & RPL9B & OST3 \\
\hline 0.0077 & SMM1 & 0.02599 & RPSOA & 0.01321 & RPS0A & PAA1 \\
\hline 0.02712 & SNF5 & 0.03142 & RPS6A & 0.02147 & RPS6A & PAC10 \\
\hline 0.0004 & SPE1 & 0.00972 & SAP155 & 0.00589 & SAP155 & PDC6 \\
\hline $1.4 \mathrm{E}-11$ & SPE2 & 0.03332 & SET6 & 0.03305 & SET6 & PH086 \\
\hline $1.9 \mathrm{E}-06$ & SPE3 & 0.03808 & SIN3 & 0.00025 & SIN3 & PNT1 \\
\hline 0.01733 & SSH1 & 0.00488 & SKO1 & 0.04681 & SK01 & REC8 \\
\hline 0.02559 & sWS2 & 0.00017 & SLG1 & $3.5 \mathrm{E}-05$ & SLG1 & RIM8 \\
\hline 0.04713 & TBS1 & 0.00022 & SMM1 & 0.00072 & SNF5 & ROX1 \\
\hline $1.9 \mathrm{E}-06$ & TC089 & 0.00114 & SNF5 & $4.9 \mathrm{E}-06$ & SPE1 & RPA34 \\
\hline 0.00822 & TEF4 & $2.50 \mathrm{E}-05$ & SPE1 & $3.8 \mathrm{E}-08$ & SPE2 & RPL11B \\
\hline 0.00049 & TGS1 & $1.48 \mathrm{E}-06$ & SPE2 & $8.2 \mathrm{E}-07$ & SPE3 & RPL16B \\
\hline 0.00895 & THR1 & 4.96E-07 & SPE3 & 0.00451 & SSD1 & RPL37B \\
\hline 2.6E-08 & THR4 & 0.01567 & SSD1 & 0.01809 & SWD1 & RPL6B \\
\hline 0.04284 & UIP4 & 0.00298 & SSH1 & 0.00233 & TC089 & RPL9B \\
\hline 0.02907 & VPS1 & 0.02748 & SWD1 & 0.02615 & TEF4 & RPS0A \\
\hline 0.00114 & VPS4 & 0.00344 & SWS2 & 0.00174 & TGS1 & RPS6A \\
\hline 0.03658 & YDC1 & 0.03969 & TBS1 & 0.00139 & THR1 & SAP155 \\
\hline 0.03277 & YDL199C & 0.00204 & TGS1 & $9.5 \mathrm{E}-07$ & THR4 & SET6 \\
\hline 0.04523 & YDR056C & 0.00011 & THR4 & 0.02946 & TRF4 & SIN3 \\
\hline 0.00953 & YDR269C & 0.00498 & TRF4 & 0.00293 & VPS4 & SK01 \\
\hline 0.02305 & YDR459C & 0.04601 & UIP4 & 0.01986 & YDC1 & SLG1 \\
\hline 0.00431 & YER066W & 0.04099 & VPS1 & 0.01982 & YDL199C & SMM1 \\
\hline 0.00177 & YFL032W & 0.0385 & YDC1 & 0.01926 & YDR056C & SNF5 \\
\hline 0.01374 & YGL261C & 0.03322 & YDR056C & 0.04055 & YDR269C & SPE1 \\
\hline 0.01722 & YGL262W & 0.01406 & YFL032W & 0.03471 & YDR459C & SPE2 \\
\hline 0.00379 & YGR107W & 0.00804 & YGL118C & 0.00259 & YER066W & SPE3 \\
\hline 0.04067 & YGR168C & 0.01854 & YGL140C & 0.0016 & YFL032W & SSD1 \\
\hline 0.04586 & YIL086C & 0.02066 & YGL177W & 0.02785 & YGL118C & SSH1 \\
\hline 0.03528 & YIL158W & 0.04415 & YGL235W & 0.0028 & YGL140C & SWD1 \\
\hline $2.5 \mathrm{E}-05$ & YJL046W & 0.00038 & YGR107W & 0.02448 & YGL177W & SWS2 \\
\hline $8.4 \mathrm{E}-06$ & YJL135W & 0.02944 & YGR168C & 0.01714 & YGL235W & TBS1 \\
\hline 0.04164 & YMR124W & 0.0409 & YIL158W & 0.04381 & YGL261C & TCO89 \\
\hline 0.00504 & YMR269W & 0.02638 & YJL046W & 0.0178 & YGL262W & TEF4 \\
\hline 0.02437 & YMR326C & 0.01895 & YJR003C & 0.0073 & YGR107W & TGS1 \\
\hline 0.00125 & YNL080C & 0.04061 & YLR446W & 0.04287 & YIL086C & THR1 \\
\hline 0.03575 & YNL146W & 0.02878 & YLR460C & 0.00106 & YJL135W & THR4 \\
\hline 0.02758 & YNL190W & 0.02881 & YMR124W & 0.00334 & YJR003C & TRF4 \\
\hline 0.04168 & YNR004W & 8.13E-08 & YMR269W & 0.01558 & YLR446W & UIP4 \\
\hline 0.01745 & YOL092W & 0.00269 & YMR326C & 0.04547 & YLR460C & VPS1 \\
\hline $3.2 \mathrm{E}-05$ & YOR008C-A & 2.78E-11 & YNL080C & 0.01789 & YNL080C & VPS4 \\
\hline 0.00168 & YOR309C & 0.04126 & YNL146W & 0.0311 & YNL146W & YDC1 \\
\hline 0.02709 & YPL102C & 0.04364 & YNL190W & 0.01758 & YNR020C & YDL199C \\
\hline 0.01382 & YPL158C & $9.17 \mathrm{E}-05$ & YNR004W & 0.00215 & YOR008C-A & YDR056C \\
\hline 0.02174 & YPL272C & 0.02135 & YNR020C & $1.8 \mathrm{E}-05$ & YOR309C & YDR269C \\
\hline \multirow{8}{*}{0.03033} & YPT11 & 0.01932 & YOL092W & 0.0173 & YPL017C & YDR459C \\
\hline & & 0.01174 & YOR008C-A & 0.00036 & YPL158C & YER066W \\
\hline & & $1.01 \mathrm{E}-06$ & YOR309C & 0.02065 & YPL182C & YFL032W \\
\hline & & 0.00738 & YPL017C & 0.03569 & YPL245W & YGL118C \\
\hline & & 0.04518 & YPL102C & 0.02222 & YPL272C & YGL140C \\
\hline & & 0.00029 & YPL158C & & & YGL177W \\
\hline & & 0.03051 & YPL182C & & & YGL235W \\
\hline & & 0.04497 & YPL245W & & & YGL261C \\
\hline
\end{tabular}




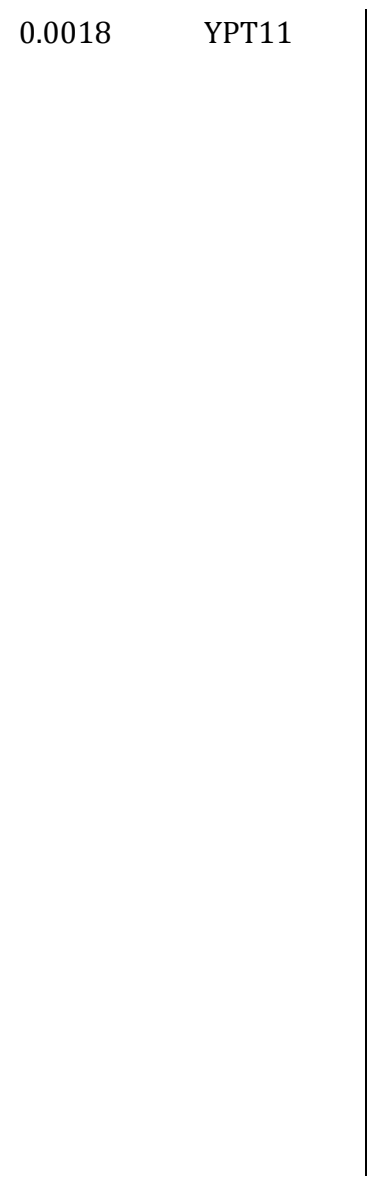

YGL262W

YGR107W

YGR168C

YIL086C

YIL158W

YJL046W

YJL135W

YJR003C

YLR446W

YLR460C

YMR124W

YMR269W

YMR326C

YNL080C

YNL146W

YNL190W

YNR004W

YNR020C

YOL092W

YOR008C-A

YOR309C

YPL017C

YPL102C

YPL158C

YPL182C

YPL245W

YPL272C

YPT11 


\section{Appendix V: Spot Dilution of Sensitive Strains}

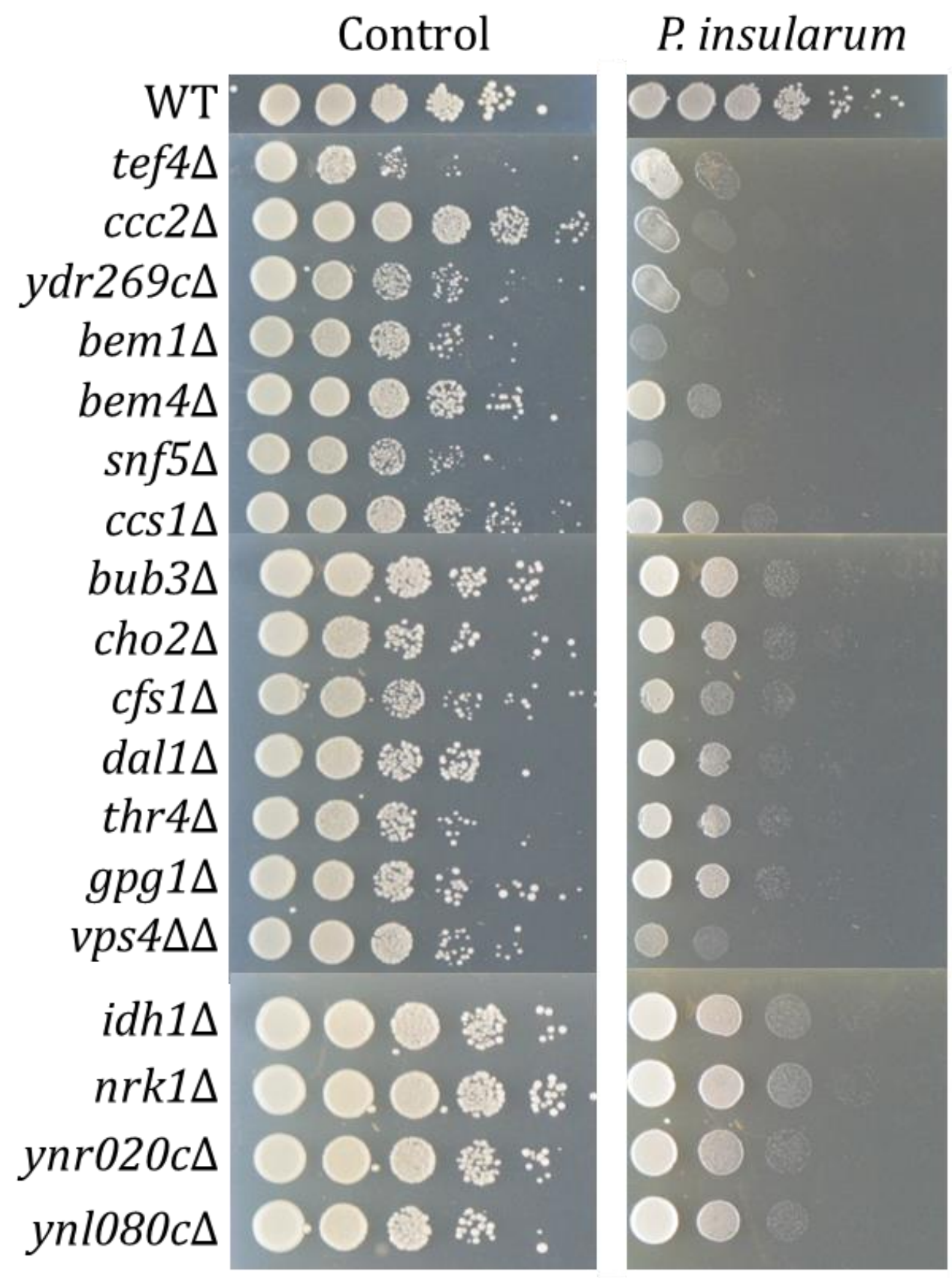




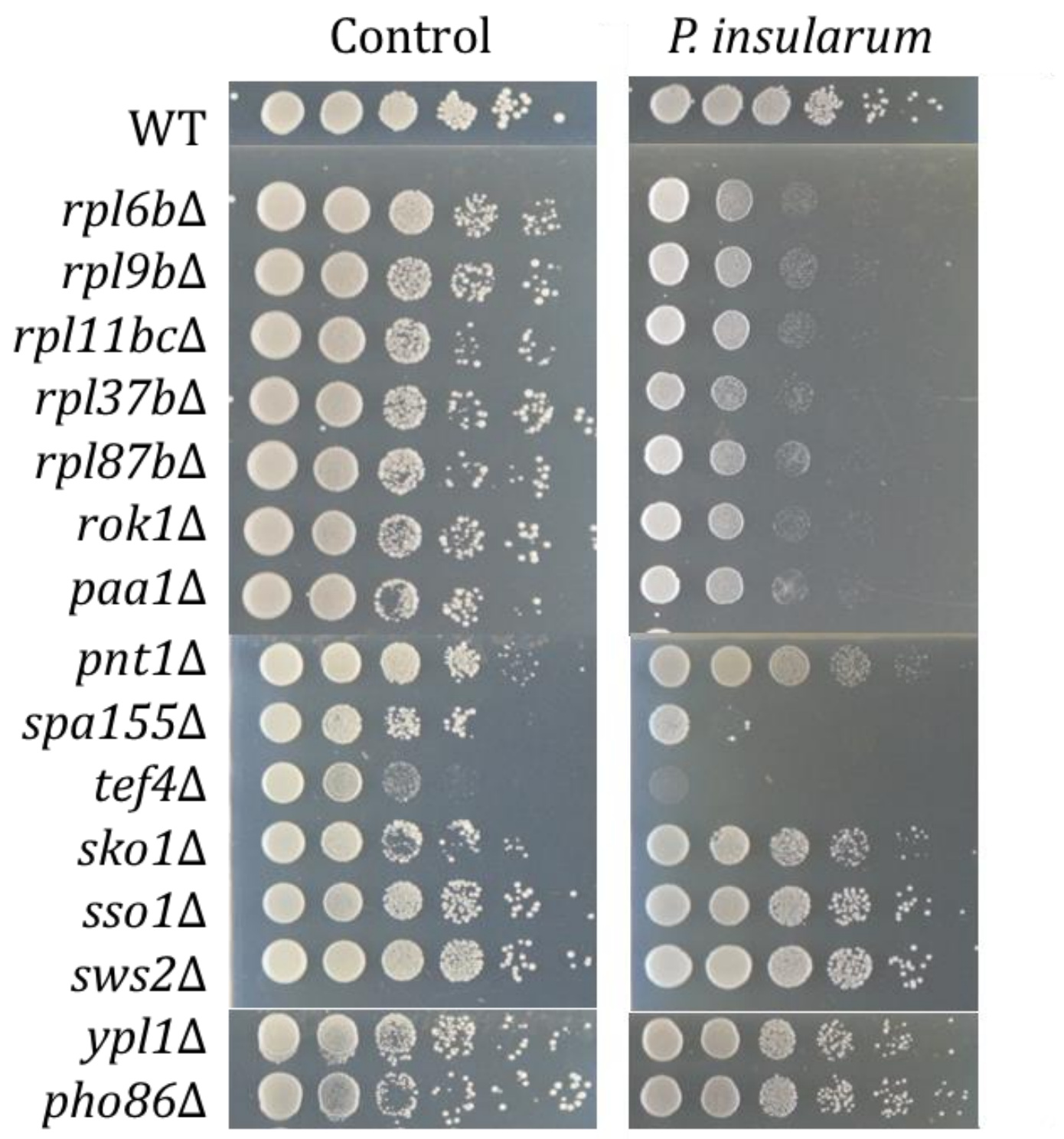




\section{Appendix VI: Script for Acapella GFP Quantification}

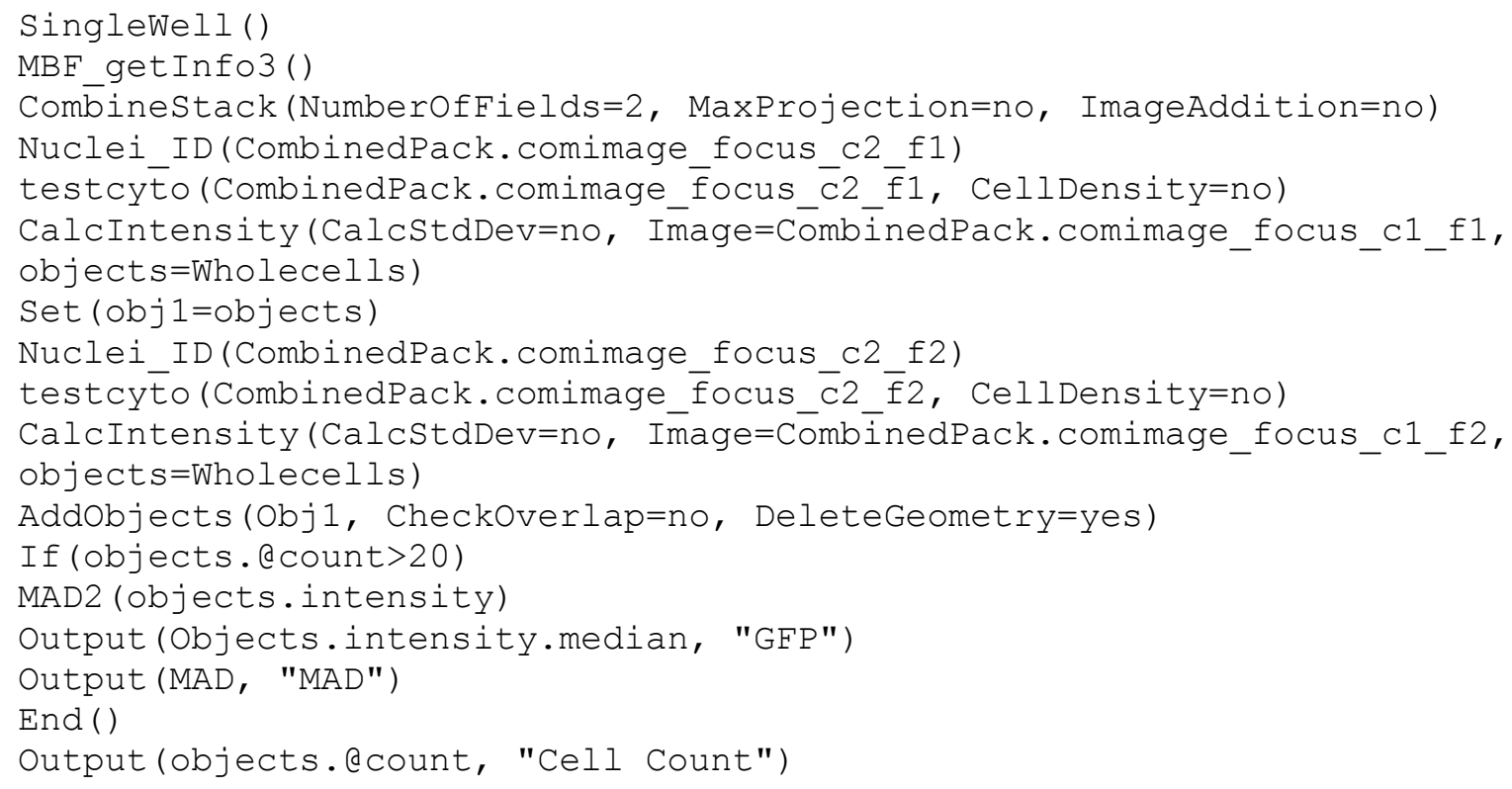




\section{Appendix VII: Optimized Concentrations for HIP/HOP}

Table A7: $\quad$ Summary of optimization of concentrations for the aqueous and methanolic extracts for pooled diploid mutants analyses for HIP and HOP profiling. Three concentrations for both the aqueous and methanolic extracts were assessed, including $0.0005 \% \mathrm{v} / \mathrm{v}, 0.001 \% \mathrm{v} / \mathrm{v}$ and $0.005 \% \mathrm{v} / \mathrm{v}$ for the aqueous extract, and $0.00005 \% \mathrm{v} / \mathrm{v}, 0.0001 \% \mathrm{v} / \mathrm{v}$ and $0.0005 \% \mathrm{v} / \mathrm{v}$ for the methanolic extract. The residual growth from pooled diploid libraries (heterozygous and homozygous) were compared to control conditions to determine the best concentration producing a growth inhibition of $10-20 \%$, or a residual growth of 90 $80 \%$. Red indicates minimal growth inhibition, blue indicates too much growth inhibition, and green indicates ideal growth inhibition.

\begin{tabular}{|c|c|c|c|c|c|c|c|}
\hline \multirow{19}{*}{ 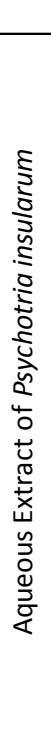 } & & \multicolumn{3}{|c|}{ Heterozygous Pool } & \multicolumn{3}{|c|}{ Homozygous Pool } \\
\hline & & Control & 0.0005 & Residual \% & Control & 0.0005 & Residual \% \\
\hline & Rep-1 & 0.574 & 0.573 & 96.0 & 0.679 & 0.667 & 103.6 \\
\hline & Rep-2 & 0.656 & 0.631 & 105.7 & 0.644 & 0.661 & 102.6 \\
\hline & Rep-3 & 0.597 & 0.541 & 90.6 & 0.621 & 0.611 & 94.9 \\
\hline & Median & 0.597 & & 96.0 & 0.644 & & 102.6 \\
\hline & StDev & & & 6.3 & & & 4.8 \\
\hline & & Control & 0.001 & Residual \% & Control & 0.001 & Residual \% \\
\hline & Rep-1 & 0.574 & 0.475 & 79.6 & 0.679 & 0.576 & 89.4 \\
\hline & Rep-2 & 0.656 & 0.506 & 84.8 & 0.644 & 0.536 & 83.2 \\
\hline & Rep-3 & 0.597 & 0.479 & 80.2 & 0.621 & 0.537 & 83.4 \\
\hline & Median & 0.597 & & 80.2 & 0.644 & & 83.4 \\
\hline & StDev & & & 2.4 & & & 3.5 \\
\hline & & Control & 0.005 & Residual \% & Control & 0.005 & Residual \% \\
\hline & Rep-1 & 0.574 & 0.343 & 57.5 & 0.679 & 0.448 & 69.6 \\
\hline & Rep-2 & 0.656 & 0.333 & 55.8 & 0.644 & 0.439 & 68.2 \\
\hline & Rep-3 & 0.597 & 0.391 & 65.5 & 0.621 & 0.409 & 63.5 \\
\hline & Median & 0.597 & & $\mathbf{5 7 . 5}$ & 0.644 & & 68.2 \\
\hline & StDev & & & 4.4 & & & 3.2 \\
\hline & & Control & 0.00005 & Residual \% & Control & 0.00005 & Residual \% \\
\hline & Rep-1 & 0.581 & 0.530 & 93.8 & 0.645 & 0.624 & 96.7 \\
\hline & Rep-2 & 0.565 & 0.551 & 97.5 & 0.611 & 0.648 & 100.5 \\
\hline$\frac{2}{5}$ & Rep-3 & 0.522 & 0.549 & 97.2 & 0.669 & 0.631 & 97.8 \\
\hline$\overline{\mathrm{G}}$ & Median & 0.565 & & 97.2 & 0.645 & & 97.8 \\
\hline 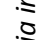 & StDev & & & 2.1 & & & 1.9 \\
\hline \pm & & Control & 0.0001 & Residual \% & Control & 0.0001 & Residual \% \\
\hline ई & Rep-1 & 0.581 & 0.461 & 81.6 & 0.645 & 0.497 & 77.1 \\
\hline$\stackrel{n}{n}$ & Rep-2 & 0.565 & 0.475 & 84.1 & 0.611 & 0.506 & 78.4 \\
\hline$\overline{0}$ & Rep-3 & 0.522 & 0.490 & 86.7 & 0.669 & 0.495 & 76.7 \\
\hline$\stackrel{0}{0}$ & Median & 0.565 & & 84.1 & 0.645 & & 77.1 \\
\hline 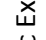 & StDev & & & 2.6 & & & 0.908 \\
\hline$\frac{\text { ou }}{0}$ & & Control & 0.005 & Residual \% & Control & 0.005 & Residual \% \\
\hline 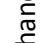 & Rep-1 & 0.581 & 0.330 & 58.4 & 0.645 & 0.401 & 62.2 \\
\hline$\stackrel{ \pm}{0}$ & Rep-2 & 0.565 & 0.364 & 64.4 & 0.611 & 0.431 & 66.8 \\
\hline & Rep-3 & 0.522 & 0.373 & 66.0 & 0.669 & 0.415 & 64.3 \\
\hline & Median & 0.565 & & 64.4 & 0.645 & & 64.3 \\
\hline & StDev & & & 4.0 & & & 2.3 \\
\hline
\end{tabular}




\section{Appendix VIII: Primer Sequences used for Bar-Seq}

Illumina P5 Primer:

5'-A ATG ATA CGG CGA CCA CCG AGA TCT ACA CTC TTT CCC TAC ACG ACG CTC TTC CGA TCT-3'

Illumina Sequencing Primer:

5'-ACA CTC TTT CCC TAC ACG ACG CTC TTC CGA TCT-3'

(Robinson et al., 2015) 


\section{Appendix IX: ICP-MS quantifying Zinc}

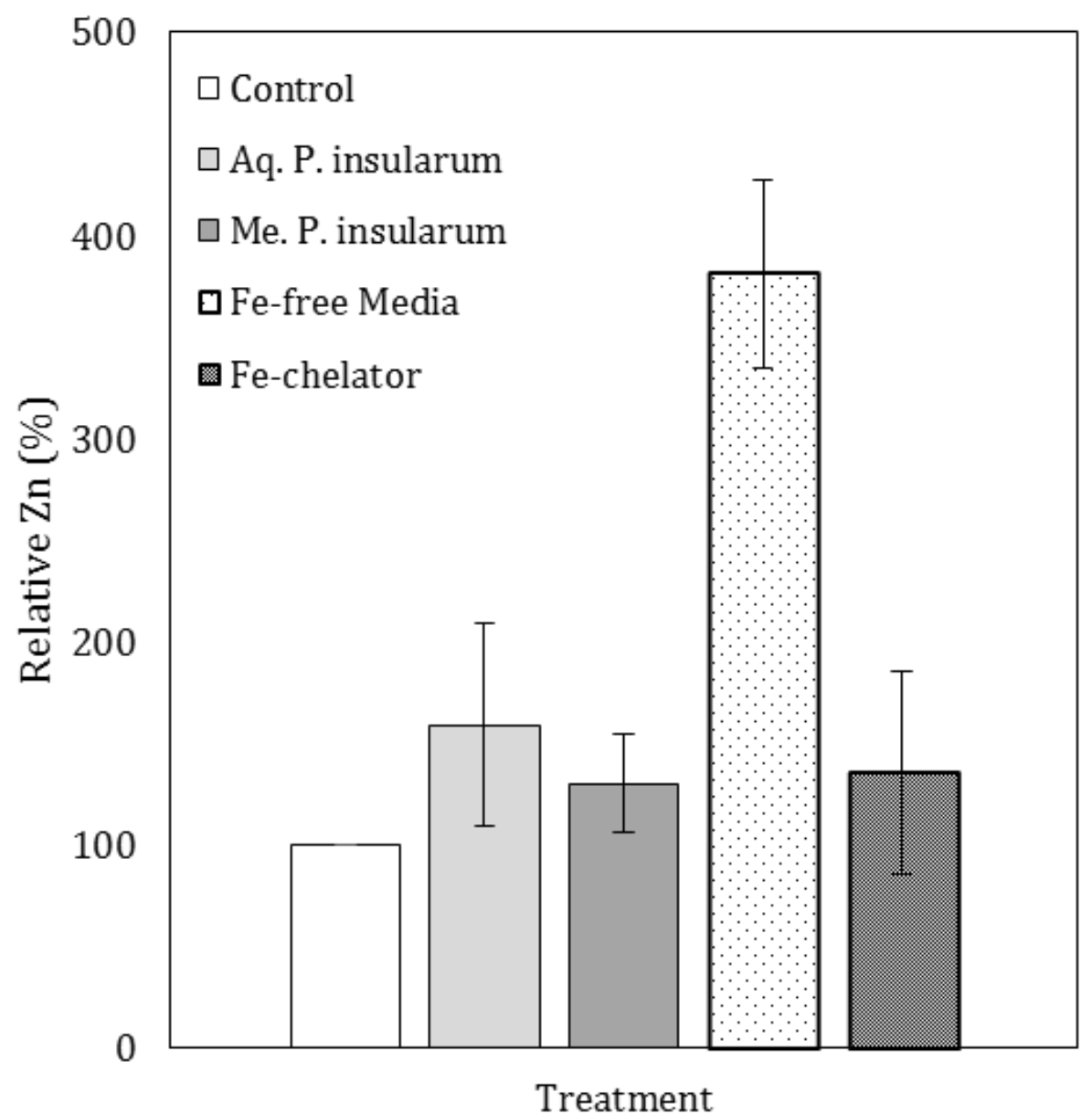

Figure A9: Quantified intracellular zinc levels in WT yeast. BY4741 cells were grown in control media (Control) as well as in treatment media containing $0.05 \% \mathrm{v} / \mathrm{v}$ of aqueous extract (Aq. P. insularum), $0.005 \% \mathrm{v} / \mathrm{v}$ of methanolic extract (Me. P. insularum), iron-free media (Fe-free media) and media containing $0.1 \mu \mathrm{M}$ bathophenanthroline disulfonic acid iron chelator control (Fe-chelator). Quantified iron is reported as relative total intracellular iron, compared to control BY4741 obtained from the average and standard deviation of two biological replicates. To determine if any increased levels of Zn were statistically significant, a one-tailed Student's t-test was carried out $(* *$ : p-value $<0.01)$. 
